Functional genetics and genomics of the banana black Sigatoka pathogen Pseudocercospora fijiensis

Caucasella Díaz-Trujillo 


\section{Thesis committee}

\section{Promotors}

Prof. Dr G.H.J. Kema

Special Professor Tropical Phytopathology

Wageningen University \& Research

Prof. Dr P.J.G.M. de Wit

Emeritus Professor of Phytopathology

Wageningen University \& Research

\section{Co-promotor}

Prof. Dr R.E. Arango

Associate professor at the National University of Colombia, School of Biosciencies, Faculty of Sciences. Colombia

Head of Plant Biotechnology Unit, Corporación para Investigaciones Biológicas (CIB).

Colombia

\section{Other members}

Prof. Dr J.A.G.M. de Visser, Wageningen University \& Research

Dr M.H. Lebrun, INRA, Thiverval-Grignon, France

Dr C. Waalwijk, Wageningen University \& Research

Dr L. De Lapeyre De Bellaire, CIRAD, Montpellier, France

This research was conducted under auspices of the Graduate School Experimental Plant Sciences 


\title{
Functional genetics and genomics of the banana black Sigatoka pathogen Pseudocercospora fijiensis
}

\author{
Caucasella Díaz-Trujillo
}

Thesis

submitted in fulfilment of the requirements for the degree of doctor at Wageningen University

by the authority of the Rector Magnificus,

Prof. Dr A.P.J. Mol,

in the presence of the

Thesis Committee appointed by the Academic Board

to be defended in public

on Wednesday 6 June 2018

at 1:30 p.m. in the Aula. 
Caucasella Díaz Trujillo

Functional genetics and genomics of the banana black Sigatoka pathogen Pseudocercospora fijiensis,

246 pages.

PhD thesis, Wageningen University, Wageningen, the Netherlands, (2018)

With references, with summaries in English and Spanish

ISBN 978-94-6332-364-2

DOI: https://doi.org/10.18174/449585 


\section{Table of content}

Chapter 1 General Introduction and outline of the thesis

Pag.

7

Chapter 2 Combating a global threat to a clonal crop: banana black Sigatoka pathogen Pseudocercospora fijiensis (synonym Mycosphaerella fijiensis) genomes reveal clues for disease control

Supplementary data

Chapter 3 Targeted and random genetic modification of the black Sigatoka pathogen Pseudocercospora fijiensis by Agrobacterium tumefaciens-mediated transformation

Chapter 4 A new mechanism for reduced sensitivity to demethylationinhibitor fungicides in the fungal banana black Sigatoka pathogen Pseudocercospora fijiensis

Supplementary data

Chapter 5 General discussion

Summary

Acknowledgments

About the author

List of publications

Experimental Plant Sciences (EPS) certificate 

CHAPTER 1

INTRODUCTION 


\section{Banana and Plantain}

Food security is a major concern with an expected increase of the global population to nine billion people in 2050 (Godfray et al., 2010). Traditionally, the major staple foods wheat, rice, and maize are considered to be of prime importance to secure sufficient food for the future (FAOSTAT, 2015). However, reducing food waste and diversifying diets are also important drivers for food security. For instance, the consumption of vegetables is very important for proteins and vitamins, but still far below the required level in many countries (Godfray et al., 2010). In addition, other crops are of prime importance as staples for millions of people. These include crops such as cassava, millet, but also bananas and plantain. Such crops are frequently called "orphan crops" as they are far less attractive for research, hence research and development are generally lagging behind compared to the aforementioned leading staples, but ironically feed millions of people (Esfeld et al., 2013). Bananas originated in Southeast Asia, where a long domestication period of wild species derived from inter- or intra crosses between the founding seeded diploid banana species Musa acuminata (AA, $2 \mathrm{n}=22$ ) and $M$. balbisiana $(\mathrm{BB}, 2 \mathrm{n}=22)$ resulted into a great diploid, triploid and tetraploid diversity with over 1,000 registered accessions. The edible sterile triploids, comprising AAA, $\mathrm{AAB}$ (plantain) and $\mathrm{ABB}$ (cooking banana), several tetraploids and parthenocarpic diploids are clonally propagated (Heslop-Harrison and Schwarzacher, 2007; Ortiz and Vuylsteke, 1994), which contributed to their dissemination into all tropical and subtropical areas. Roughly, local varieties are the preferred fruits and staples at domestic markets, whereas the AAA triploids such as "Cavendish" are the preferred commodity (Valmayor et al., 2000) that is produced across all tropical and sub-tropical environments with Ecuador as the prime exporter (Table 1, FAOSTAT, 2015). In short, banana is a major crop in the developing economies of Asia, Africa and Latin America for both domestic and export markets. Over $80 \%$ of the global production is locally consumed and just 17 million tonnes (18\%) with a 
monetary value of 29.3 billion US\$, is representing the top fruit in Western supermarkets (FAOSTAT, 2015).

Table 1. Major producers, exporters and importers of banana and plantain

\begin{tabular}{lrlrlr}
\multicolumn{2}{c}{$\begin{array}{c}\text { Producers* } \\
\text { Million metric tonnes }\end{array}$} & \multicolumn{1}{c}{ Exporters*** $^{*}$} & \multicolumn{2}{c}{ Importers*** } & $(\%)$ \\
India & 26.217 & Ecuador & 23.3 & United States & 18.8 \\
Philippines & 8.687 & Guatemala & 10.5 & Belgium & 8.8 \\
China & 8.042 & Costa Rica & 8.5 & Russia & 7.1 \\
China mainland & 7.834 & Belgium & 8.0 & Germany & 7.1 \\
Brazil & 6.998 & Colombia & 7.8 & Japan & 6.6 \\
Ecuador & 6.701 & Philippines & 5.3 & United Kingdom & 5.9 \\
Indonesia & 6.005 & Netherlands & 3.8 & China & 4.2 \\
Guatemala & 2.448 & Dominican Republic & 3.8 & Netherlands & 3.7 \\
United Republic & 2.447 & United States & 3.7 & Italy & 3.5 \\
of Tanzania & & & & & \\
Mexico & 2.151 & Ivory Coast & 3.1 & France & 3.3 \\
Others & 62.675 & Others & 22.2 & Others & 31.0 \\
\hline \multicolumn{2}{c}{ Sources: FAOSTAT2015. Data from 2014* } & &
\end{tabular}

www.worldtopexports.com. Data from 2016 **

The sterile - thus seedless - triploid edible bananas, such as the "Cavendish" varieties, are vigorous high-yielding plants that are easily propagated and have an acceptable taste. The tailored logistic chain seamlessly provides millions of bananas on a daily basis to Western consumers. Cavendish varieties dominate the export trade due to their resistance to Panama disease or Fusarium wilt that is caused by the soil-borne fungus Fusarium oxysporum f. sp. cubense (Foc). The so-called Race 1 strains of this fungus (Ordonez et al., 2015) wiped out its predecessor, the "Gros Michel" bananas in Latin America in the previous century (Ploetz, $2015,2006)$. As a result, the transnational export companies were gradually changing to the less preferred, but resistant "Cavendish" clones, a process strongly intermingled with politics and societal unrest (Koeppel, 2008). Despite their resistance to Panama disease, "Cavendish" bananas are highly susceptible to black Sigatoka, a destructive foliar blight caused by the Dothideomycete Pseudocercospora fijiensis (previously Mycosphaerella fijiensis Morelet). 
This disease is a major concern to the industry as the foliage has to be disease free to avoid premature ripening which turns the crop unfit for export. Since "Cavendish" bananas are very susceptible to $P$. fijiensis, fungicides are a cornerstone for global export banana production (Lepoivre P., 2003; Marín et al., 2003). In addition, other pathogens and pests challenge bananas wherever they are grown, including fungal, bacterial and viral disease as well as a range of nematode and insect pests. Nematodes are important soil-borne threats of banana, primarily the endoparasitic Radopholus similis, which is the most damaging species worldwide, along with other species such as Helicotylenchus spp., root-lesion and root knot nematodes from the genera Pratylenchus and Meloidogyne, respectively (Speijer and De Waele, 1997).

The banana weevil Cosmopolites sordidus is the most serious insect pest in most production environments, but thrips species cause major cosmetic damage to the fruit for the export trade (Dubois and Coyne, 2011; Gold et al., 2001). Besides, stem borers, moths, beetles, fruitflies and whiteflies are significant pests (Gold et al., 2001). The latter two excrete honeydew, facilitating foliar and fruit molds, thereby reducing the market value. Aphids often transmit viruses such as banana bunchy top virus (BBTV), which is a major constraint to production in several African countries. The disease can be prevented with regular inspections and destruction of infected plants (Kumar et al., 2015), but extension frequently fails and hence local epidemics jeopardize production. Banana streak virus (BSV) is a complex of different circular dsDNA episomal viral species that are integrated in the genomes of banana varieties with the B genome (Iskra-Caruana et al., 2014).

In the group of bacterial diseases, Xanthomonas wilt, caused by Xanthomonas campestris, Moko disease, caused by Ralstonia solanacearum biovar 1 race 2, and brown rot caused by Ralstonia solanacearum biovar 1, race 1, are major threats to bananas and plantains. They are dispersed by insects and contaminated tools worldwide, but their 
incidence is remarkably irregular (Tinzaara et al., 2006). Recent advances in breeding and genetic engineering provide new options for disease control (Tripathi et al., 2014).

As mentioned before, with respect to fungal disease, "Cavendish" quenched the Panama disease epidemic (Ploetz, 2006). However, since the 1960 another Foc strain colloquially called Tropical Race 4 (TR4) - has emerged that now develops into a pandemic thereby threatening the entire "Cavendish" production, which comprises approximately $40 \%$ of the global production with immense importance for domestic and export markets. Due to the "orphan crop" label, investment in research and development has been minimal over the past decades and hence, there is no commercially viable replacement for these varieties. In addition, TR4 also kills many local varieties. Thus "Cavendish", once a blessing, now is a vehicle for disaster. Lack of attention, unawareness of the problem and poor vision have left growers with unmanageable disease problems and therefore, new initiatives are urgently required that professionalize the development of new plant material through advanced breeding programs. Until new varieties reach the market, bluntly, exclusion is basically the only effective control strategy for the majority of banana disease and pests.

\section{Pseudocercospora fijiensis}

The black Sigatoka fungus $P$. fijiensis was originally described by Rhodes (1964) in the Sigatoka district, on Viti Levu, Fiji islands. Together with $P$. musae (previously $M$. musicola) causal agent of Sigatoka disease, and P. eumusae (previously M. eumusae) that causes Septoria leaf spot, they form the Sigatoka complex, as these pathogens can coexist on the same leaf or in the same lesion even along with other fungi of minor economic importance (Arzanlou et al., 2008). Sigatoka pathogens diminish photosynthetic capacity due to leaf necrosis (Lepoivre P., 2003; Marín et al., 2003; Okole and Schulz, 1997), induce early 


\section{CHAPTER 1}

ripening and reduce bunch weight up to $40 \%$ (Castelan et al., 2012) unless crop protection agents are used.

The main dispersing propagules of this bipolar heterothallic pathogen are the sexual air-borne ascospores, and the asexual conidiospores, which are continuously produced under field conditions. The disease cycle starts when spores land on the abaxial side of the youngest "cigar" leaf. Therefore, disease symptoms initially appear at the perimeter of the leaf towards the midrib. Under favorable conditions $\left(>25^{\circ} \mathrm{C}\right.$ temperature, $95 \%$ relative humidity for at least 72h) spores germinate, grow epiphytically and enter the leaf through stomata and subsequently, hyphae colonize the apoplastic space of the mesophyll and accumulate in the substomatal cavities to produce the fructifications thereby gradually forming chlorotic lesion in the foliage that eventually expand into large coalescing necrotic blotches. According to Meredith and Lawrence (1969) and Fouré (1985), disease development can be divided into six stages. The first necrotic spots appear at the abaxial leaf side, not even visible by transmittent light. When spots enlarge parallel to the veins, they become visible also at the adaxial side, which is distinctive of stage 2 and sporodochia are generated to produce and release conidia on conidiophores, which emerge through stomatal openings at both sides of the leaf. Conidia disperse typically just a few meters by wind or splash as well as by contact with other leaves. Once streaks coalesce to form larger and ticker streak lesions, stage 3 is reached. In the meantime, sporodochia develop spermagonia with spermatia that are required for the sexual reproduction. At stage 4 lesions on both leaf sides darken and at stage 5 these lesions become depressed and surrounded by a yellow halo. Eventually, lesions develop into paper-like dry and whitish/greyish colored blotches, surrounded by a dark border and the yellow halo that over time coalesce into large necrotic blotches with dry and inner gray zones bearing the perithecia that are visible as minute dark spots on both sides of the foliage. They discharge air-borne ascospores upon drastic changes in relative humidity, which disseminate over 
relatively short distances (Burt et al., 1999; Rieux et al., 2014) and start a new disease cycle (Figure 1).

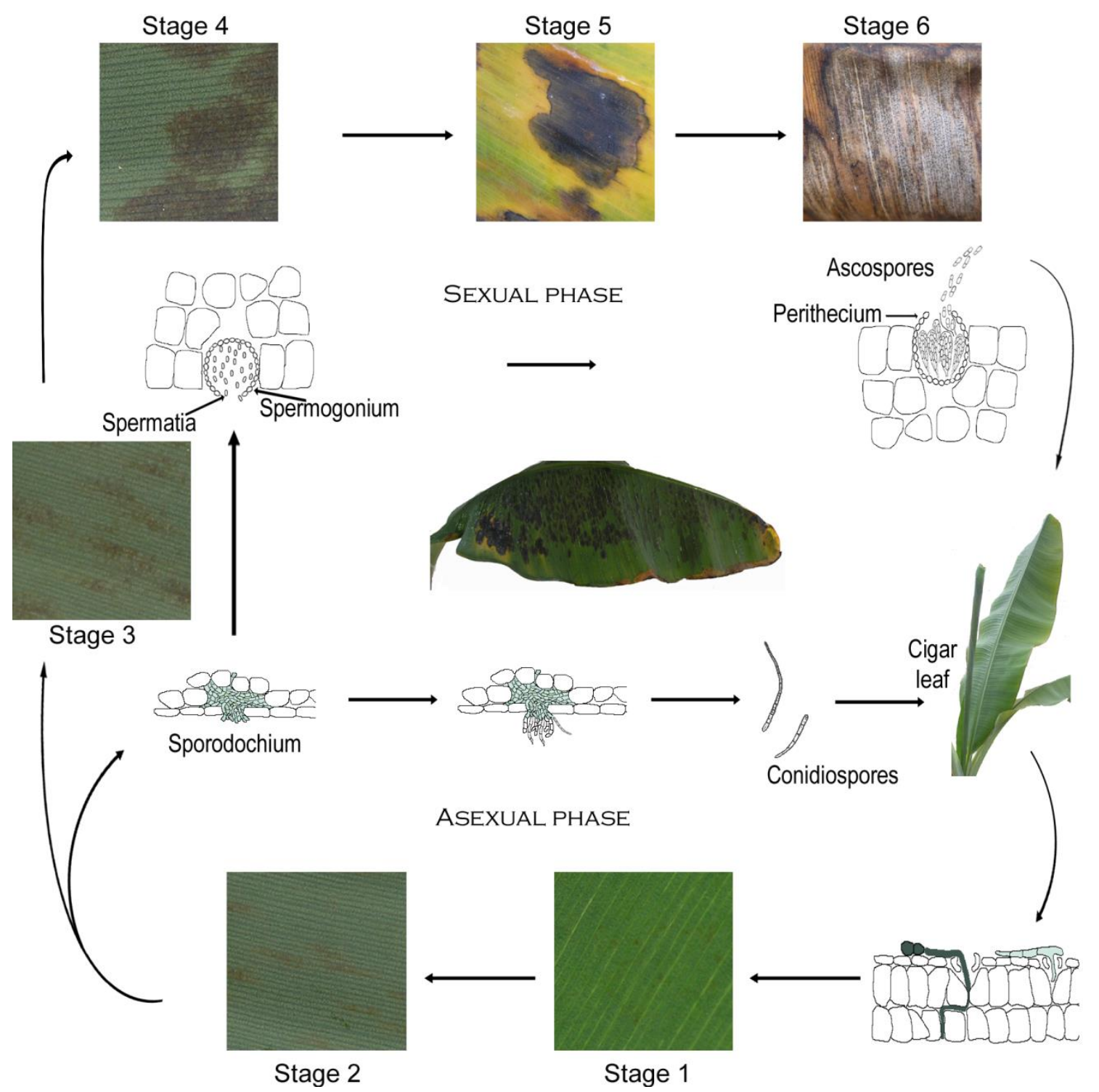

Figure 1. The black Sigatoka disease cycle.

The evolution of the Sigatoka complex results in visually undistinguishable symptoms of the individual Pseudocercospora species. Yellow Sigatoka spread globally but was gradually outcompeted by the more aggressive black Sigatoka disease. Presently, $P$. musae is confined to highland regions $>1000 \mathrm{~m}$ with a few exceptions (Amil et al., 2007), whereas $P$. fijiensis has spread from Fiji (Rhodes P.L., 1964) to almost all tropic and subtropical areas 


\section{CHAPTER 1}

where bananas are grown and is still migrating to new areas, for instance in Brazil where the Northeast region is still not affected due to a "buffer zone" that is already affected (Brito et al., 2015), and in Australia where it was eradicated during an intensive campaign (NSW Primary Industries, 2013). Field populations of $P$. fijiensis are genetically extremely diverse due to their bipolar heterothallic mating system (Carlier, 1994; Chong, 2016; Conde-Ferráez et al., 2007). Yet, the global population can be divided into four main groups: Southeast Asia, Pacific Islands, Africa and Latin America that all trace back to the gene center of banana in Southeast Asia (Carlier, 1994; Carlier et al., 1996). Founder effects of these populations have shown that the dissemination of $P$. fijiensis was mainly due to human transport of contaminated plant material, as often leaves are used for different activities, such as food wrapping and transport of the fruit. Thus the independent introductions in Latin America and Africa in 1972 and 1973, respectively, generated two genetically highly diverging populations. At the continental scale, stochastic spreading has been suggested by either a limited natural dispersal of ascospores or by transport of infected material (Carlier et al., 1996; Rivas et al., 2004). Most of these conclusions were derived from extensive populations genetic analyses by using a wide array of genetic markers, which comprise restriction fragment polymorphisms (RFLPs) (Carlier, 1994; Carlier et al., 1996; Hayden and Carlier, 2003), AFLP, SSRs, Microsatellites (Mueller R. et al., 1995; Neu C. et al., 1999); PCR-RFLP (Fahleson et al., 2009; Hayden and Carlier, 2003; Zapater et al., 2004), and recently also genome sequence based markers including single nucleotide polymorphisms (SNPs) (Zandjanakou-Tachin et al., 2009), mini satellites and variable numbers of tandem repeats (VNTRs) (Chong, 2016; Garcia et al., 2010). These data were used to scrutinize the structure of local populations and underscored the huge diversity at all levels, even between isolates derived from one lesion (Arzanlou et al., 2007; Müller R. et al., 1995; Müller et al., 1997), 
which was also reported for the related Zymoseptoria tritici pathogen of wheat (Linde et al., 2002).

The third species of the Sigatoka complex, P. eumusae is spreading towards Asia and Africa (Carlier et al., 2000; Crous and Mourichon, 2002) and is considered to be more aggressive than P. fijiensis (Zandjanakou-Tachin et al., 2013). As mentioned above, these Pseudocercospora species trigger similar symptoms in banana and plantain leaves, are morphologically similar and often coexist in the same leaf (Carlier et al., 2000; Crous and Mourichon, 2002). Pseudocercospora fijiensis can be clearly differentiated from P. musae with genetic markers, based on the ITS1 region (Johanson and Jeger, 1993). In 2007, TaqMan markers were identified by Arzanlou et al. (2007), which quantitatively detect the individual species. Recently, Chang et al. (2016) showed that the infection biology of P. eumusae slightly differs from $P$. fijiensis and $P$. musae. These data, altogether, contribute to a better understanding of the Sigatoka complex and its control in banana and plantain.

\section{Control of Pseudocercospora fijiensis}

The management of $P$. fijiensis in banana is challenging because export bananas are typically cultivated in large monocultures. Despite the use of different control strategies, including good field practices such as early warning/forecasting systems, symptoms screening, biocontrol agents and leaf pruning, the only truly effective black Sigatoka management relies on frequent fungicide applications (Chong, 2016). Evidently, such frequent applications - usually $>50$ times per year - drive selection in pathogen populations, requiring even more applications that eventually spiral into unmanageable disease situations and a fall back to protectants that threaten the often precautious tropical environments and plantation workers even more and take a $30 \%$ share of the overall production costs (Chong, 
2016; FRAC, 2010; Marín et al., 2003). The versatility of the fungus to maintain genetic diversity through its continuous sexual reproduction complicates disease control due to an unparalleled trend towards reduced sensitivity. Therefore, complex spraying schedules are developed to minimize this trend. These comprise a range of chemistries, including protectants such as benomyl (Stover R.H., 1990), chlorothalonil and the dithiocarbamates mancozeb (Romero and Sutton, 1997) that are considered as "low-resistance" risk products (FRAC, 2010). Besides, they include systemic fungicides, such as the demethylase inhibitors (DMIs) and strobilurins (Amil et al., 2007; Sierotzki et al., 2000). Despite the reduced sensitivity to DMIs in most production environments they are still the cornerstone of black Sigatoka disease management (Cañas-Gutiérrez et al., 2009; Marín et al., 2003). Clearly, there is a great need to better understand the mechanisms of reduced sensitivity, which is essential to develop better management strategies. The Fungicide Resistance Action Committee (FRAC) banana working group monitors and evaluates control strategies and recommends on active ingredients and application strategies to minimize sensitivity loss. Recent research described a global sensitivity map base on analyses of the Pfcyp51 gene, which is the target for DMIs (Cañas-Gutiérrez et al., 2009; Chong, 2016). Interestingly, a recent market pull also favored alternative technologies and production areas enabling the export of organic bananas that are grown in areas which are not conducive for P. fijiensis, such as in Peruvian highlands, and hence do not require fungicide applications (USDA, 2008). The demand and export of such organic bananas annually increases up to $19 \%$ according to "Peru: organic banana exports grow by 19\%" (2016). 


\section{Host resistance to Pseudocercospora fijiensis}

As mentioned above, the predecessors of the commercial banana and plantain varieties are the seeded diploids $M$. acuminata and M. balbisiana. Edible banana, however, share one common denominator, which is seedlessness, either due to triploidy or parthenocarpy (Heslop-Harrison and Schwarzacher, 2007; Perrier et al., 2011; Sardos et al., 2016). Clearly, breeding bananas requires seed production and smart strategies to eventually develop seedless, edible fruits while capturing genetic diversity. Yet, low fertility, triploidy, slow propagation, space requirement and cycling time all contributed to a complex process that has not attracted manifold breeders and hence, the release of new attractive germplasm has been minimal over the last decades (Ploetz et al., 2015). Therefore, crop improvement has largely concentrated on selection within clones, for instance for productivity, fruit flavor, hardiness, height, bunch weight and shelf life. Evidently, under such conditions and circumstances, on top of the "orphan status" of banana, progress on the genetic control of fungal disease has been extremely slow, and with regard to black Sigatoka, hardly any progress has been realized. As a matter of fact, not a single gene for resistance to black Sigatoka has been identified, which is an important basis for improving resistance to this disease. Genetic analyses that have used natural inoculum for phenotyping segregating populations are essentially useless due to the known genetic diversity of natural populations and the huge effect of fluctuating environmental factors (Ortiz and Vuylsteke, 1995). In conclusion, the very basic work for understanding the banana-black Sigatoka pathosystem has still to start. The current thesis is a humble effort to begin developing the required basis for advanced (population) genetics and genomics studies of $P$. fijiensis to close existing knowledge gaps supporting the development of a more sustainable banana production.

Fullerton and Olsen (1995) were the first to challenge a differential set of banana and plantain accessions with individual $P$. fijiensis isolates from three global populations and 


\section{CHAPTER 1}

compared these data with previous field performance. Such data are basic, yet crucial to understand diversity. Other, previously considered notoriously difficult pathosystems such as Leptosphaeria maculans-Brassica napus (Balesdent et al., 2002) and Zymoseptoria triticiwheat (Brading et al., 2002; Ghaffary et al., 2011; Habig et al., 2017; Kema et al., 2018; Kema et al., 2000; Mirzadi Gohari et al., 2015), are current model systems that are grounded in large host-pathogen matrices that eventually resulted in the identification of a range of resistance genes that are applied in contemporary advanced breeding programs (Goodwin, 2007; Kohli and Skovmand, 1997; Rouxel and Balesdent, 2013). Phenotyping diversity in the banana- $P$. fijiensis pathosystem is evidently more complicated, hence it would benefit from a smart, simplified selection process, for instance by using effectors. Previously, highly resistant, partially resistant or susceptible responses to $P$. fijiensis have been reported (Fouré et al., 1990; Mourichon et al., 1987), and cytological studies revealed that only the resistant host displayed an immediate response upon stomatal penetration (Beveraggi et al., 1995), suggesting a gene-for-gene relationship and partial resistance between $P$. fijiensis and "Yangambi Km5" and "Fougamou", respectively. However, the resistance of "Yangambi Km5" was eventually circumvented, even without commercial exploitation and increased selection pressure, which eventually resulted in establishing the International Musa testing program (ITMP). It initially comprised hybrids from the Honduras Foundation for Agricultural Research (FHIA) and a differential set of standard clones that were planted at geographically different locations in Latin America and Africa (Jones, 1994). Data analyses identified $M$. acuminata spp. burmannicoides "Calcutta 4" as a reference for resistance to $P$. fijiensis (Table 2; Jones and Tézenas du Montcel, 1994).

Table 2. Results of the International Musa testing program, Phase 1 surveying for resistance to black Sigatoka (INIBAP, 1992). 


\begin{tabular}{|c|c|c|c|c|}
\hline Accessions & $\begin{array}{c}\text { International } \\
\text { Transit } \\
\text { Centre Code }\end{array}$ & $\begin{array}{l}\text { Genome } \\
\text { formula }\end{array}$ & Type & $\begin{array}{c}\text { Response to } \\
\text { Black Sigatoka }\end{array}$ \\
\hline Tuu Gia & 0610 & AA & Edible cultivar & ER \\
\hline $\begin{array}{l}\text { Musa acuminata spp. } \\
\text { burmannicoides }\end{array}$ & 0249 & AA & Wild species & ER \\
\hline Musa acuminata spp. malaccensis & 0609 & AA & Wild species & ER \\
\hline Pisang Lilin & 0001 & AA & Edible cultivar & HR \\
\hline Pisang Berlin & 0611 & AA & Edible cultivar & $\mathrm{R}$ \\
\hline Pisang Mas & 0653 & AA & Edible cultivar & $\mathrm{R}$ \\
\hline Musa balbisiana & 0405 & $\mathrm{BB}$ & Wild species & $\mathrm{R}$ \\
\hline SF215/NBA14 & 0267 & AA & Edible cultivar & S \\
\hline Niyarma Yik & 0269 & AA & Edible cultivar & HS \\
\hline
\end{tabular}

\begin{tabular}{|c|c|c|c|}
\hline $\begin{array}{l}\text { ER: Extremely } \\
\text { resistant }\end{array}$ & $\begin{array}{l}\text { HR: Highly } \\
\text { resistant }\end{array}$ & R: Resistant & S: Susceptible \\
\hline
\end{tabular}

In the pre-genomic era, attempts to identify and use phytotoxins (Molina, 1989; Okole and Schulz, 1997; Stierle et al., 1991; Strobel et al., 1992; Upadhyay et al., 1990) tried to avoid the necessary aforementioned groundwork, but did contribute to a deeper understanding of pathogenesis on resistant and susceptible banana varieties. More recently, Hoss et al. (2000) found that 2,4,8-trihydrotetralone accumulates in susceptible bananas just before severe disease symptoms develop, while the timing in resistant bananas occurred during the very early stage of infection. Finally, additional attempts for resistance screening included the use of juglone, other toxins and secondary metabolites belonging to phenylpropanoid pathway (Chuc-Uc et al., 2011; Cruz-Cruz et al., 2009; Hadrami et al., 2005; Otálvaro et al., 2007), suggesting phytoalexins to play a role. Molecular analyses have also discovered a first insight in the defensive arsenal of banana and include the generation of $\mathrm{H}_{2} \mathrm{O}_{2}$ (Beltrán-García et al., 2014; Cavalcante et al., 2011; Torres et al., 2012), peroxidase, phenylalanine amonia lyase, $\beta$ 1,3-glucanase (Torres et al., 2012) and chitinases (Escobar-Tovar et al., 2015a; Torres et al., 
2012). However, as pointed out above, the major missing yet required link is a better understanding of the genetics of resistance and pathogenicity. Unveiling the genetic basis of resistance in "Calcutta 4" is still pending, which is mostly due to the poor amenability of $P$. fijiensis. The genome sequencing of M. acuminata ssp. malaccensis (D’Hont et al., 2012) and M. balbisiana (Davey et al., 2013) are, along with classical analyses and genome wide association studies, promising turning points for gene discovery.

\section{Genomics, genetics and gene expression of Pseudocercospora fijiensis}

The release of the draft $P$. fijiensis genome sequence was an immediate resource for multiple comparative analyses (Arzanlou et al., 2010; Chang et al., 2016; Chong, 2016; Churchill, 2011; Couoh-Uicab et al., 2013; de Wit et al., 2012; Escobar-Tovar et al., 2015a; Kantún-Moreno et al., 2013; Noar and Daub, 2016a, 2016b; Ohm et al., 2012; Stergiopoulos et al., 2010, 2014). The complete genome sequence (Arango Isaza et al., 2016), was until recently the largest Dothideomycete genome, which is presently exceeded by the $>150 \mathrm{Mb}$ genome of the only mycorrhizal species Cenococcum geophilum (Peter et al., 2016) in this class of fungi. Genome expansion is largely due to repetitive elements in both species. It was intriguing that once the $P$. fijiensis genome was released, the first homologue of the Cladosporium fulvum effector Avr4 was discovered (Stergiopoulos et al., 2010). The heterologously produced PfAvr4 effector as well as most Avr4 isoforms, triggered hypersensitive response on tomato leaves carrying the cognate $C f 4$ resistance gene (Kombrink, 2012; Stergiopoulos et al., 2014, 2010).

Clearly, the fungal and host genome sequences helped to reveal the first glimpses of the banana- $P$. fijiensis interaction, but many questions remain unresolved. The abovementioned initial effector analysis, two fungal linkage maps for genome assembly 
(Arango Isaza et al., 2016), a detailed global analysis of DMI sensitivity (Chong, 2016) and several expression analyses involving glycosyl phosphatidyl-inositol (GTI) proteins (KantúnMoreno et al., 2013) and a putative ABC transporter - the orthologue of the MgAtr4 in Z. tritici - (Couoh-Uicab et al., 2013) are resulting milestones. Even more so, once such studies are lined-up with the infection process which is accompanied by differential accumulation of melanin and $\mathrm{H}_{2} \mathrm{O}_{2}$ (Beltrán-García et al., 2014; Torres et al., 2012). Recent analysis of Escobar et al. (2015a) and Chang et al. (2016) suggest that pathogenesis is aided by an overall stealth weakening of host tissue rather than a rapid collapse, which accords with the known pathogenesis in other Dothideomycetes (Goodwin et al., 2011; Ohm et al., 2012).

With the advance of genome analyses, hence gene discovery, tools for functional analyses become indispensable. Several protocols have been published (Balint-Kurti et al., 2001; Escobar-Tovar et al., 2015b) that enable random mutagenesis, but do not facilitate functional analyses. Only recently, Onyilo et al., (2017) reported the first gene targeted by silencing in $P$. fijiensis; a very powerful transient transformation method particularly for reversible and/or incomplete gene expression prevention.

In this thesis I describe the genome sequence of $P$. fijiensis, a protocol for its transformation and the application in tool development for functional assays. Together, these should aid the advance of (functional) genetics in this important banana pathogen, thereby contributing to the overall aim of a more sustainable and diversified banana production. 


\section{Outline}

Overall, at the start of my PhD thesis project I identified major bottlenecks to advance research in the banana - P. fijiensis pathosystem. Firstly, I considered that sequencing the genome of $P$. fijiensis would be a powerful base to increase the understanding of its (infection) biology. Secondly, I contemplated on the possibility to develop a protocol for homologous recombination, which would facilitate functional analysis of any given gene. Hence, my thesis was divided into five chapters.

Chapter 1 introduces the crop banana and plantain, presents their importance and describes the major biological threats of the crop with an emphasis on the fungal diseases to eventually focus on black Sigatoka. The biology of its causal agent P. fijiensis and the interaction with banana are briefly described. Furthermore, a critical analysis of the current standing in banana and black Sigatoka research is provided, thereby indicating the major bottlenecks. I considered that resolving the latter would bring banana research to another level.

Chapter 2 describes the genome sequencing of $P$. fijiensis, which resulted in a $74 \mathrm{Mb}$, genome size, mostly containing repetitive DNA and a remarkable differential GC content, which was also observed in the other Sigatoka complex constituents $P$. musae and $P$. eumusae. Characteristics of gene content, gene models, synteny, gene expression, and genome dynamics are compared with the Dothideomycete reference genome of Zymoseptoria tritici, as well as with other Dothideomycetes, and discussed in relation to its life style. An initial functional analysis of PfAvr4 is included, which is indicative for gene-for-gene interaction in the banana - P. fijiensis pathosystem. Finally, the genome information was used to analyze strobilurin fungicide resistance dynamics in natural $P$. fijiensis populations in various banana plantations in Costa Rica. 
Chapter 3 focuses on developing a protocol for Agrobacterium-mediated transformation (ATMT) of P. fijiensis. Initially, I developed a protocol for random mutagenesis and generated GFP- and Ds Red- labeled strains. Subsequently, I modified the protocol for targeted mutagenesis and used it for functional analysis of Pfavr4 and for developing a $P f k u 70$ knock-out strain. The latter is expected to have an impaired nonhomologous end joining pathway, which would increase homologous recombination and thereby enhance the throughput for homologous recombination and hence, developing targeted knock-out strains.

Chapter 4 describes the use of the aforementioned transformation protocol to analyze the mechanism of DMI sensitivity in P. fijiensis. Besides abundant mutations in the coding sequence of the target Pfcyp51 gene, we discovered multiple repeated insertions in the Pfcyp51 promoter region that are crucial for reduced sensitivity of $P$. fijiensis resistance to triazoles. The promoter sequences were detected in various international field strains with reduced sensitivity to propiconazole, difenoconazole and epoxiconazole as well as in field strains from Costa Rican farms, which suggests that these mutants were selected by the frequent fungicide applications in these plantations. ATMT was used to replace the promotor of a sensitive strain by the promotor of a resistant strain to show that both structural variants are required for reduced sensitivity.

Finally, Chapter 5 provides a general and critical treatise of the achieved results and brings a wider scope for forthcoming research that is necessary to improve our understanding of the banana - P. fijiensis pathosystem and to develop strategies that contribute to a sustainable banana production. 
References

Amil, A.F., Heaney, S.P., Stanger, C., Shaw, M.W., 2007. Dynamics of QoI sensitivity in Mycosphaerella fijiensis in Costa Rica during 2000 to 2003. Phytopathology 97, 1451-1457. https://doi.org/10.1094/PHYTO-97-11-1451

Arango Isaza, R.E., Díaz-Trujillo, C., Dhillon, B., Aerts, A., Carlier, J., Crane, C.F., Jong, T.V. de, Vries, I. de, Dietrich, R., Farmer, A.D., Fortes Fereira, C., Garcia, S., Guzmán, M., Hamelin, R.C., Lindquist, E.A., Mehrabi, R., Quiros, O., Schmutz, J., Shapiro, H., Reynolds, E., Scalliet, G., Souza Jr, M., Stergiopoulos, I., Van der Lee, T.A.J., Wit, P.J.G.M. de, Zapater, M.-F., Zwiers, L.-H., Grigoriev, I.V., Goodwin, S.B., Kema, G.H.J., 2016. Combating a global threat to a clonal crop: the banana black Sigatoka pathogen Pseudocercospora fijiensis (synonym Mycosphaerella fijiensis) genomes reveals clues for disease control. PLoS Genet In press.

Arzanlou, M., Abeln, E.C.A., Kema, G.H.J., Waalwijk, C., Carlier, J., Vries, I. de, Guzmán, M., Crous, P.W., 2007. Molecular diagnostics for the Sigatoka disease complex of banana. Phytopathology 97, 1112-1118. https://doi.org/10.1094/PHYTO-97-9-1112

Arzanlou, M., Crous, P.W., Zwiers, L.-H., 2010. Evolutionary dynamics of mating-type loci of Mycosphaerella spp. occurring on banana. Eukaryot. Cell 9, 164-172. https://doi.org/10.1128/EC.00194-09

Arzanlou, M., Groenewald, J.Z., Fullerton, R.A., Abeln, E.C.A., Carlier, J., Zapater, M.-F., Buddenhagen, I.W., Viljoen, A., Crous, P.W., 2008. Multiple gene genealogies and phenotypic characters differentiate several novel species of Mycosphaerella and related anamorphs on banana. Persoonia Mol. Phylogeny Evol. Fungi 20, 19-37. https://doi.org/10.3767/003158508X302212

Balesdent, M.H., Attard, A., Kühn, M.L., Rouxel, T., 2002. New avirulence genes in the phytopathogenic fungus Leptosphaeria maculans. Phytopathology 92, 1122-1133. https://doi.org/10.1094/PHYTO.2002.92.10.1122

Balint-Kurti, P.J., May, G.D., Churchill, A.C.L., 2001. Development of a transformation system for Mycosphaerella pathogens of banana: a tool for the study of host/pathogen interactions. FEMS Microbiol. Lett. 195, 9-15. https://doi.org/10.1111/j.1574-6968.2001.tb10490.x

Beltrán-García, M.J., Prado, F.M., Oliveira, M.S., Ortiz-Mendoza, D., Scalfo, A.C., Pessoa, A., Jr, Medeiros, M.H.G., White, J.F., Di Mascio, P., 2014. Singlet molecular oxygen generation by light-activated dhn-melanin of the fungal pathogen Mycosphaerella fijiensis in black Sigatoka disease of bananas. PLoS ONE 9, e91616. https://doi.org/10.1371/journal.pone.0091616

Beveraggi, A., Mourichon, X., Sallé, G., 1995. Étude comparée des premières étapes de l'infection chez des bananiers sensibles et résistants infectés par le Cercospora fijiensis (Mycosphaerella fijiensis) agent responsable de la maladie des raies noires. Can. J. Bot. 73, 1328-1337. https://doi.org/10.1139/b95-144

Brading, P.A., Verstappen, E.C.P., Kema, G.H.J., Brown, J.K.M., 2002. A gene-for-gene relationship between wheat and Mycosphaerella graminicola, the Septoria tritici blotch pathogen. Phytopathology 92, 439-445. https://doi.org/10.1094/PHYTO.2002.92.4.439

Brito, F.S.D., Fraaije, B., Miller, R.N.G., 2015. Sigatoka disease complex of banana in Brazil: Management practices and future directions. Outlooks Pest Manag. 26, 78-81. https://doi.org/10.1564/v26_apr_08

Burt, P.J.A., Rosenberg, L.J., Rutter, J., Ramirez, F., Gonzales O, H., 1999. Forecasting the airborne spread of Mycosphaerella fijiensis, a cause of black Sigatoka disease on banana: estimations of numbers of perithecia and ascospores. Ann. Appl. Biol. 135, 369-377. https://doi.org/10.1111/j.1744-7348.1999.tb00863.x

Cañas-Gutiérrez, G.P., Angarita-Velásquez, M.J., Restrepo-Flórez, J.M., Rodríguez, P., Moreno, C.X., Arango, R., 2009. Analysis of the CYP51 gene and encoded protein in propiconazole-resistant isolates of Mycosphaerella fijiensis. Pest Manag. Sci. 65, 892-899. https://doi.org/10.1002/ps.1770 
Carlier, J., 1994. DNA Restriction fragment length polymorphisms in Mycosphaerella species that cause banana leaf spot diseases. Phytopathology 84, 751. https://doi.org/10.1094/Phyto-84751

Carlier, J., Lebrun, M.H., Zapater, M.F., Dubois, C., Mourichon, X., 1996. Genetic structure of the global population of banana black leaf streak fungus, Mycosphaerella fijiensis. Mol. Ecol. 5, 499-510. https://doi.org/10.1111/j.1365-294X.1996.tb00342.x

Carlier, J., Zapater, M.-F., Lapeyre, F., Jones, D.R., Mourichon, X., 2000. Septoria leaf spot of banana: a newly discovered disease caused by Mycosphaerella eumusae (Anamorph Septoria eumusae). Phytopathology 90, 884-890. https://doi.org/10.1094/PHYTO.2000.90.8.884

Castelan, F.P., Saraiva, L.A., Lange, F., de Lapeyre de Bellaire, L., Cordenunsi, B.R., Chillet, M., 2012. Effects of black leaf streak disease and Sigatoka disease on fruit quality and maturation process of bananas produced in the subtropical conditions of southern Brazil. Crop Prot. 35, 127-131. https://doi.org/10.1016/j.cropro.2011.08.002

Cavalcante, M. de J.B., Escoute, J., Madeira, J.P., Romero, R.E., Nicole, M.R., Oliveira, L.C., Hamelin, C., Lartaud, M., Verdeil, J.L., 2011. Reactive oxygen species and cellular interactions between Mycosphaerella fijiensis and banana. Trop. Plant Biol. 4, 134-143. https://doi.org/10.1007/s12042-011-9071-8

Chang, T.-C., Salvucci, A., Crous, P.W., Stergiopoulos, I., 2016. Comparative genomics of the Sigatoka disease complex on banana suggests a link between parallel evolutionary changes in Pseudocercospora fijiensis and Pseudocercospora eumusae and increased virulence on the banana host. PLOS Genet. 12, e1005904. https://doi.org/10.1371/journal.pgen.1005904

Chong, P., 2016. Origin, versatility and distribution of azole fungicide resistance in the banana black Sigatoka pathogen Pseudocercospora fijiensis. Wageningen University, Wageningen, the Netherlands.

Chuc-Uc, J., Brito-Argáez, L., Canto-Canché, B., Tzec-Simá, M., Rodríguez-García, C., PerazaEcheverría, L., Peraza-Echeverría, S., James-Kay, A., Cruz-Cruz, C.A., Peña-Rodríguez, L.M., Islas-Flores, I., 2011. The in vitro secretome of Mycosphaerella fijiensis induces cell death in banana leaves. Plant Physiol. Biochem. PPB Société Fr. Physiol. Végétale 49, 572 578. https://doi.org/10.1016/j.plaphy.2011.02.006

Churchill, A.C.L., 2011. Mycosphaerella fijiensis, the black leaf streak pathogen of banana: progress towards understanding pathogen biology and detection, disease development, and the challenges of control. Mol. Plant Pathol. 12, 307-328. https://doi.org/10.1111/j.13643703.2010.00672.x

Conde-Ferráez, L., Waalwijk, C., Canto-Canché, B. B., Kema, G.H.J., Crous, P.W., James, A.C., Abeln, E.C.A. 2007. Isolation and characterization of the mating type locus of Mycosphaerella fijiensis, the causal agent of black leaf streak disease of banana. Mol. Plant Pathol. 8: 111-120.

Couoh-Uicab, Y., Islas-Flores, L., Kantún-Moreno, N., Zwiers, L.H., Tzec-Simá, M., Echeverría, S.P.-, Brito-Argáez, L., Peraza-Echeverría, L., Grijalva-Arango, R., James, A., Rodríguez-García, C., Canto-Canché, B., 2013. Cloning, in silico structural characterization and expression analysis of MfAtr4, an ABC transporter from the banana pathogen Mycosphaerella fijiensis. Afr. J. Biotechnol. 11, 54-79. https://doi.org/10.4314/ajb.v11i1.

Cruz-Cruz, C.A., García-Sosa, K., Escalante-Erosa, F., Peña-Rodríguez, L.M., 2009. Production of hydrophilic phytotoxins by Mycosphaerella fijiensis. J. Gen. Plant Pathol. 75, 191-195. https://doi.org/10.1007/s10327-009-0165-1

Davey, M.W., Gudimella, R., Harikrishna, J.A., Sin, L.W., Khalid, N., Keulemans, J., 2013. A draft Musa balbisiana genome sequence for molecular genetics in polyploid, inter- and intraspecific Musa hybrids. BMC Genomics 14, 683. https://doi.org/10.1186/1471-2164-14-683

de Wit, P.J.G.M., van der Burgt, A., Okmen, B., Stergiopoulos, I., Abd-Elsalam, K.A., Aerts, A.L., Bahkali, A.H., Beenen, H.G., Chettri, P., Cox, M.P., Datema, E., de Vries, R.P., Dhillon, B., Ganley, A.R., Griffiths, S.A., Guo, Y., Hamelin, R.C., Henrissat, B., Kabir, M.S., Jashni, M.K., Kema, G., Klaubauf, S., Lapidus, A., Levasseur, A., Lindquist, E., Mehrabi, R., Ohm, R.A., Owen, T.J., Salamov, A., Schwelm, A., Schijlen, E., Sun, H., van den Burg, H.A., van Ham, R.C.H.J., Zhang, S., Goodwin, S.B., Grigoriev, I.V., Collemare, J., Bradshaw, R.E., 2012. The Genomes of the fungal plant pathogens 
Cladosporium fulvum and Dothistroma septosporum reveal adaptation to different hosts and lifestyles but also signatures of common ancestry. PLoS Genet. 8. https://doi.org/10.1371/journal.pgen.1003088

D'Hont, A., Denoeud, F., Aury, J.-M., Baurens, F.-C., Carreel, F., Garsmeur, O., Noel, B., Bocs, S., Droc, G., Rouard, M., Da Silva, C., Jabbari, K., Cardi, C., Poulain, J., Souquet, M., Labadie, K., Jourda, C., Lengellé, J., Rodier-Goud, M., Alberti, A., Bernard, M., Correa, M., Ayyampalayam, S., Mckain, M.R., Leebens-Mack, J., Burgess, D., Freeling, M., Mbéguié-A-Mbéguié, D., Chabannes, M., Wicker, T., Panaud, O., Barbosa, J., Hribova, E., Heslop-Harrison, P., Habas, R., Rivallan, R., Francois, P., Poiron, C., Kilian, A., Burthia, D., Jenny, C., Bakry, F., Brown, S., Guignon, V., Kema, G., Dita, M., Waalwijk, C., Joseph, S., Dievart, A., Jaillon, O., Leclercq, J., Argout, X., Lyons, E., Almeida, A., Jeridi, M., Dolezel, J., Roux, N., Risterucci, A.-M., Weissenbach, J., Ruiz, M., Glaszmann, J.-C., Quétier, F., Yahiaoui, N., Wincker, P., 2012. The banana (Musa acuminata) genome and the evolution of monocotyledonous plants. Nature 488, 213-217. https://doi.org/10.1038/nature11241

Dubois, T., Coyne, D.L., 2011. Integrated peset management of banana, in: Banana Breeding: Progress and Challenges. CRC Press, USA, pp. 121-144.

Escobar-Tovar, L., Guzmán-Quesada, M., Sandoval-Fernández, J.A., Gómez-Lim, M.A., 2015a. Comparative analysis of the in vitro and in planta secretomes from Mycosphaerella fijiensis isolates. Fungal Biol. 119, 447-470. https://doi.org/10.1016/j.funbio.2015.01.002

Escobar-Tovar, L., Magaña-Ortíz, D., Fernández, F., Guzmán-Quesada, M., SandovalFernández, J.A., Ortíz-Vázquez, E., Loske, A.M., Gómez-Lim, M.A., 2015b. Efficient transformation of Mycosphaerella fijiensis by underwater shock waves. J. Microbiol. Methods 119, 98-105. https://doi.org/10.1016/j.mimet.2015.10.006

Esfeld, K., Uauy, C., Tadele, Z., 2013. Application of TILLING for orphan crop improvement, in: Biotechnology of neglected and underutilized crops. Springer, pp. 83-113.

Fahleson, J., Nakyanzi, M., Okori, P., Seal, S., Kenyon, L., Dixelius, C., 2009. Genetic analysis of Mycosphaerella fijiensis in the Ugandan Lake Victoria region. Plant Pathol. 58, 888-897. https://doi.org/10.1111/j.1365-3059.2009.02099.x

FAOSTAT, 2015.

Fouré E., 1985. Les cercosporioses du bananier et leurs traitements. Etude de la sensibilité varietale des bananiers et plantains á Mycosphaerella fijiensis Morelet au Gabon (maladie des raies noires). III: Comportement des variétés. Fruits 40, 393-399.

Fouré, E., Mouliom-Pefoura, A., Mourichon, X., 1990. Etude de la sensibilité variétale des bananiers plantains á Mycosphaerella fijiensis au Cameroun. Caractérisation de la résistance au champs de bananiers appartenant á drivers groupes génétiques. Fruits 45, 329-338.

FRAC, 2010. FRAC REcommendations for Fungicide Mixtures - January 2010.

Fullerton, R.A., Olsen, T.L., 1995. Pathogenic variability in Mycosphaerella fijiensis Morelet, cause of black Sigatoka in banana and plantain. N. Z. J. Crop Hortic. Sci. 23, 39-48. https://doi.org/10.1080/01140671.1995.9513866

Garcia, S. a. L., Van der Lee, T. a. J., Ferreira, C.F., Te Lintel Hekkert, B., Zapater, M.-F., Goodwin, S.B., Guzmán, M., Kema, G.H.J., Souza, M.T., 2010. Variable number of tandem repeat markers in the genome sequence of Mycosphaerella fijiensis, the causal agent of black leaf streak disease of banana (Musa spp). Genet. Mol. Res. GMR 9, 2207-2212. https://doi.org/10.4238/vol9-4gmr934

Ghaffary, S.M.T., Robert, O., Laurent, V., Lonnet, P., Margalé, E., Lee, T.A.J. van der, Visser, R.G.F., Kema, G.H.J., 2011. Genetic analysis of resistance to septoria tritici blotch in the French winter wheat cultivars Balance and Apache. Theor. Appl. Genet. 123, 741-754. https://doi.org/10.1007/s00122-011-1623-7

Godfray, H.C.J., Beddington, J.R., Crute, I.R., Haddad, L., Lawrence, D., Muir, J.F., Pretty, J., Robinson, S., Thomas, S.M., Toulmin, C., 2010. Food Security: The Challenge of Feeding 9 Billion People. Science 327, 812-818. https://doi.org/10.1126/science.1185383

Gold, C.S., Pena, J.E., Karamura, E.B., 2001. Biology and integrated pest management for the banana weevil Cosmopolites sordidus (Germar) (Coleoptera: Curculionidae). Integr. Pest Manag. Rev. 6, 79-155. https://doi.org/10.1023/A:1023330900707 
Goodwin, S.B., 2007. Back to basics and beyond: increasing the level of resistance to Septoria tritici blotch in wheat. Australas. Plant Pathol. 36, 532-538. https://doi.org/10.1071/AP07068

Goodwin, S.B., Ben M'Barek, S., Dhillon, B., Wittenberg, A.H.J., Crane, C.F., Hane, J.K., Foster, A.J., Van der Lee, T.A.J., Grimwood, J., Aerts, A., Antoniw, J., Bailey, A., Bluhm, B., Bowler, J., Bristow, J., van der Burgt, A., Canto-Canché, B., Churchill, A.C.L., Conde-Ferràez, L., Cools, H.J., Coutinho, P.M., Csukai, M., Dehal, P., De Wit, P., Donzelli, B., van de Geest, H.C., van Ham, R.C.H.J., Hammond-Kosack, K.E., Henrissat, B., Kilian, A., Kobayashi, A.K., Koopmann, E., Kourmpetis, Y., Kuzniar, A., Lindquist, E., Lombard, V., Maliepaard, C., Martins, N., Mehrabi, R., Nap, J.P.H., Ponomarenko, A., Rudd, J.J., Salamov, A., Schmutz, J., Schouten, H.J., Shapiro, H., Stergiopoulos, I., Torriani, S.F.F., Tu, H., de Vries, R.P., Waalwijk, C., Ware, S.B., Wiebenga, A., Zwiers, L.-H., Oliver, R.P., Grigoriev, I.V., Kema, G.H.J., 2011. Finished genome of the fungal wheat pathogen Mycosphaerella graminicola reveals dispensome structure, chromosome plasticity, and stealth pathogenesis. PLoS Genet 7, e1002070. https://doi.org/10.1371/journal.pgen.1002070

Habig, M., Quade, J., Stukenbrock, E.H., 2017. Forward genetics approach reveals host genotypedependent importance of accessory chromosomes in the fungal wheat pathogen Zymoseptoria tritici. mBio 8, e01919-17. https://doi.org/10.1128/mBio.01919-17

Hadrami, A.E., Kone, D., Lepoivre, P., 2005. Effect of juglone on active oxygen species and antioxidant enzymes in susceptible and partially resistant banana cultivars to black leaf streak disease. Eur. J. Plant Pathol. 113, 241-254. https://doi.org/10.1007/s10658-005-8675-y

Hayden, H.L., Carlier, J., 2003. Genetic structure of Mycosphaerella fijiensis populations from Australia, Papua New Guinea and the Pacific Islands. Plant Pathol. 52, 703-712. https://doi.org/10.1111/j.1365-3059.2003.00883.x

Heslop-Harrison, J.S., Schwarzacher, T., 2007. Domestication, genomics and the future for banana. Ann. Bot. 100, 1073-1084. https://doi.org/10.1093/aob/mcm191

Hoss, R., Helbig, J., Bochow, H., 2000. Function of host and fungal metabolites in resistance response of banana and plantain in the black Sigatoka disease pathosystem (Musa spp. Mycosphaerella fijiensis). J. Phytopathol. 148, 387-394. https://doi.org/10.1046/j.14390434.2000.00530.x

Iskra-Caruana, M.-L., Chabannes, M., Duroy, P.-O., Muller, E., 2014. A possible scenario for the evolution of Banana streak virus in banana. Virus Res. 186, 155-162. https://doi.org/10.1016/j.virusres.2014.01.005

Johanson, A., Jeger, M.J., 1993. Use of PCR for detection of Mycosphaerella fijiensis and M. musicola, the causal agents of Sigatoka leaf spots in banana and plantain. Mycol. Res. 97, 670-674. https://doi.org/10.1016/S0953-7562(09)80145-7

Jones D.R., 1994. The improvement and testing of Musa: a global partnership, Jones, D.R. ed, Proceedings of the first global conference of the international Musa testing program held at FHIA, Honduras. International Network for the Improvement of Banana and Plantain, FHIA, Honduras.

Jones, D.R., Tézenas du Montcel, H., 1994. Final report for UNDP/World Bank on the results of the International Musa Testing Programme (Phase I). Montpellier, France.

Kantún-Moreno, N., Vázquez-Euán, R., Tzec-Simá, M., Peraza-Echeverría, L., GrijalvaArango, R., Rodríguez-García, C., James, A.C., Ramírez-Prado, J., Islas-Flores, I., Canto-Canché, B., 2013. Genome-wide in silico identification of GPI proteins in Mycosphaerella fijiensis and transcriptional analysis of two GPI-anchored $\beta-1,3-$ glucanosyltransferases. Mycologia 105, 285-296. https://doi.org/10.3852/12-103

Kema, G.H.J., Mirzadi-Gohari A., Aouini, L., Gibriel, H.A.Y., Ware, S.B., van den Bosh, F., Manning-Smith, R., Alonso-Chavez, V., Helps, J., Ben M'Barek, S., Mehrabi, R., DiazTrujillo, C., Zamani, E., Schouten, H.J., van der Lee, T.A.J., Waalwijk, C., de Waard. M.A., de Wit, P.J.G.M., Verstappen, EC.P., Thoma, B.P.H.J., Meijer, H.J.G., Seidl, M.F. 2018. Stress and sexual reproduction affect th dynamics of the wheat pathogen effector AvrStb6 and strobilurin resistance. Nature Genet. In press. 
Kema, G.H., Verstappen, E.C., Waalwijk, C., 2000. Avirulence in the wheat septoria tritici leaf blotch fungus Mycosphaerella graminicola is controlled by a single locus. Mol. PlantMicrobe Interact. MPMI 13, 1375-1379. https://doi.org/10.1094/MPMI.2000.13.12.1375

Koeppel, D., 2008. Banana: The fate of the fruit that changed the world. Penguin Books, USA.

Kohli, M.M., Skovmand, B., 1997. Wheat varieties of South America : names, parentage, pedigrees, and origins. CIMMYT.

Kombrink, A., 2012. Heterologous production of fungal effectors in Pichia pastoris. Methods Mol. Biol. Clifton NJ 835, 209-217. https://doi.org/10.1007/978-1-61779-501-5_13

Kumar, P.L., Selvarajan, R., Iskra-Caruana, M.-L., Chabannes, M., Hanna, R., 2015. Biology, etiology, and control of virus diseases of banana and plantain. Adv. Virus Res. 91, 229-269. https://doi.org/10.1016/bs.aivir.2014.10.006

Lepoivre P., B.J.P., 2003. Lepoivre P., Busogoro J. P., Etame J. J., El Hadrami A., Carlier J., Harelimana G., Mourichon X., Panis B., Stella-Riveros A., Salle G., Strosse H., and Swennen R. (2003) Banana-Mycosphaerella fijiensis interactions. In: Mycosphaerella leaf spot diseases of bananas: present status and outlook. Proceedings of the 2nd International workshop on Mycosphaerella leaf spot diseases held in San José, Costa Rica, May 20-23, 2002. 151-159.

Linde, C.C., Zhan, J., McDonald, B.A., 2002. Population Structure of Mycosphaerella graminicola: from lesions to continents. Phytopathology 92, 946-955. https://doi.org/10.1094/PHYTO.2002.92.9.946

Marín, D.H., Romero, R.A., Guzmán, M., Sutton, T.B., 2003. Black Sigatoka: An increasing threat to banana cultivation. Plant Dis. 87, 208-222. https://doi.org/10.1094/PDIS.2003.87.3.208

Meredith, D.S., Lawrence J.S., 1969. Black leaf streak disease of bananas (Mycosphaerella fijiensis): Symptoms of disease in Hawaii, and notes on the conidial state of the causal fungus. Trans. Br. Mycol. Soc. 52, 459-476.

Mirzadi Gohari, A., Ware, S.B., Wittenberg, A.H.J., Mehrabi, R., Ben M'Barek, S., Verstappen, E.C.P., van der Lee, T.A.J., Robert, O., Schouten, H.J., de Wit, P.P.J.G.M., Kema, G.H.J., 2015. Effector discovery in the fungal wheat pathogen Zymoseptoria tritici. Mol. Plant Pathol. 16, 931-945. https://doi.org/10.1111/mpp.12251

Molina, G.C., 1989. A phytotoxic activity in extracts of broth cultures of Mycosphaerella fijiensis var. difformis and its use to evaluate host resistance to black Sigatoka. Plant Dis. 73, 142. https://doi.org/10.1094/PD-73-0142

Mourichon, X., Peter, D., Zapater, M.F., 1987. Inoculation expérimentale de M. fijiensis Morelet sur jeunes plantules de bananiers issues de culture in vitro. Fruits 42, 195-198.

Mueller R., Pasberg-Gauhl C., Kaemmer D., Kahl G., 1995. Tracing microsatellite polymorphisms within the Nigerian population of Mycosphaerella fijiensis. Infomusa 4, 9-11.

Müller, R., Pasberg-Gauhl, C., Gauhl, F., Ramser, J., Kahl, G., 1997. Oligonucleotide fingerprinting detects genetic variability at different levels in Nigerian Mycosphaerella fijiensis. J. Phytopathol. 145, 25-30. https://doi.org/10.1111/j.1439-0434.1997.tb00337.x

Neu C., Kaemmer D., Kahl G., Fisher D., Weising K., 1999. Polymorphic microsatellite markers for the banana pathogen Mycosphaerella fijiensis. Mol. Ecol. 8, 513-525.

Noar, R.D., Daub, M.E., 2016a. Bioinformatics prediction of polyketide synthase gene clusters from Mycosphaerella fijiensis. PloS One 11, e0158471. https://doi.org/10.1371/journal.pone.0158471

Noar, R.D., Daub, M.E., 2016b. Transcriptome sequencing of Mycosphaerella fijiensis during association with Musa acuminata reveals candidate pathogenicity genes. BMC Genomics 17, 690. https://doi.org/10.1186/s12864-016-3031-5

NSW Primary Industries, 2013. Exotic pest alert: black Sigatoka (No. Primefact 1250). Plant Biosecurity Orange. NSW Government, Australia.

Ohm, R.A., Feau, N., Henrissat, B., Schoch, C.L., Horwitz, B.A., Barry, K.W., Condon, B.J., Copeland, A.C., Dhillon, B., Glaser, F., Hesse, C.N., Kosti, I., LaButti, K., Lindquist, E.A., Lucas, S., Salamov, A.A., Bradshaw, R.E., Ciuffetti, L., Hamelin, R.C., Kema, G.H.J., Lawrence, C., Scott, J.A., Spatafora, J.W., Turgeon, B.G., de Wit, P.J.G.M., Zhong, S., Goodwin, S.B., Grigoriev, I.V., 2012. Diverse lifestyles and strategies of plant 
pathogenesis encoded in the genomes of eighteen dothideomycetes fungi. PLoS Pathog 8, e1003037. https://doi.org/10.1371/journal.ppat.1003037

Okole, B.N., Schulz, F.A., 1997. Selection of Mycosphaerella fijiensis-resistant cell lines from microcross sections of banana and plantain. Plant Cell Rep. 16, 339-343. https://doi.org/10.1007/BF01088293

Onyilo, F., Tusiime, G., Chen, L.-H., Falk, B., Stergiopoulos, I., Tripathi, J.N., Tushemereirwe, W., Kubiriba, J., Changa, C., Tripathi, L., 2017. Agrobacterium tumefaciens-Mediated transformation of Pseudocercospora fijiensis to determine the role of PfHog1 in osmotic stress regulation and virulence modulation. Front. Microbiol. 8. https://doi.org/10.3389/fmicb.2017.00830

Ordonez, N., Seidl, M.F., Waalwijk, C., Drenth, A., Kilian, A., Thomma, B.P.H.J., Ploetz, R.C., Kema, G.H.J., 2015. Worse comes to worst: bananas and Panama Disease-when plant and pathogen clones meet. PLOS Pathog. 11, e1005197. https://doi.org/10.1371/journal.ppat.1005197

Ortiz, R., Vuylsteke, D., 1995. Genotype-by-environment interaction in Musa germplasm revealed by multi-site evaluation in Sub-saharan Africa. HortScience 30, 795-795.

Ortiz, R., Vuylsteke, D., 1994. Inheritance of black Sigatoka disease resistance in plantain-banana (Musa spp.) hybrids. Theor. Appl. Genet. 89, 146-152. https://doi.org/10.1007/BF00225134

Otálvaro, F., Nanclares, J., Vásquez, L.E., Quiñones, W., Echeverri, F., Arango, R., Schneider, B., 2007. Phenalenone-type compounds from Musa acuminata var. "Yangambi km 5" (AAA) and their activity against Mycosphaerella fijiensis. J. Nat. Prod. 70, 887-890. https://doi.org/10.1021/np070091e

Perrier, X., De Langhe, E., Donohue, M., Lentfer, C., Vrydaghs, L., Bakry, F., Carreel, F., Hippolyte, I., Horry, J.-P., Jenny, C., Lebot, V., Risterucci, A.-M., Tomekpe, K., Doutrelepont, H., Ball, T., Manwaring, J., de Maret, P., Denham, T., 2011. Multidisciplinary perspectives on banana (Musa spp.) domestication. Proc. Natl. Acad. Sci. 108, 11311-11318. https://doi.org/10.1073/pnas.1102001108

Peter, M., Kohler, A., Ohm, R.A., Kuo, A., Krützmann, J., Morin, E., Arend, M., Barry, K.W., Binder, M., Choi, C., Clum, A., Copeland, A., Grisel, N., Haridas, S., Kipfer, T., LaButti, K., Lindquist, E., Lipzen, A., Maire, R., Meier, B., Mihaltcheva, S., Molinier, V., Murat, C., Pöggeler, S., Quandt, C.A., Sperisen, C., Tritt, A., Tisserant, E., Crous, P.W., Henrissat, B., Nehls, U., Egli, S., Spatafora, J.W., Grigoriev, I.V., Martin, F.M., 2016. Ectomycorrhizal ecology is imprinted in the genome of the dominant symbiotic fungus Cenococcum geophilum. Nat. Commun. 7, 12662. https://doi.org/10.1038/ncomms12662

Ploetz, R.C., 2015. Fusarium wilt of banana. Phytopathology 105, 1512-1521. https://doi.org/10.1094/PHYTO-04-15-0101-RVW

Ploetz, R.C., 2006. Fusarium wilt of banana is caused by several pathogens referred to as Fusarium oxysporum f. sp. cubense. Phytopathology 96, 653-656. https://doi.org/10.1094/PHYTO-960653

Ploetz, R.C., Kema, G.H.J., Ma, L.-J., 2015. Impact of diseases on export and smallholder production of banana. Annu. Rev. Phytopathol. 53, 269-288. https://doi.org/10.1146/annurevphyto-080614-120305

Rhodes P.L., 1964. A new banana disease in Fiji. Commonealth Phytopathol. News 10, 38-41.

Rieux, A., Soubeyrand, S., Bonnot, F., Klein, E.K., Ngando, J.E., Mehl, A., Ravigne, V., Carlier, J., de Lapeyre de Bellaire, L., 2014. Long-distance wind-dispersal of spores in a fungal plant pathogen: Estimation of anisotropic dispersal kernels from an extensive field experiment. PLOS ONE 9, e103225. https://doi.org/10.1371/journal.pone.0103225

Rivas, G.-G., Zapater, M.-F., Abadie, C., Carlier, J., 2004. Founder effects and stochastic dispersal at the continental scale of the fungal pathogen of bananas Mycosphaerella fijiensis. Mol. Ecol. 13, 471-482. https://doi.org/10.1046/j.1365-294X.2003.02043.x

Romero, R.A., Sutton, T.B., 1997. Sensitivity of Mycosphaerella fijiensis, causal agent of black Sigatoka of banana, to propiconazole. Phytopathology 87, 96-100. https://doi.org/10.1094/PHYTO.1997.87.1.96 
Rouxel, T., Balesdent, M.-H., 2013. From model to crop plant-pathogen interactions: cloning of the first resistance gene to Leptosphaeria maculans in Brassica napus. New Phytol. 197, 356-358. https://doi.org/10.1111/nph.12099

Sardos, J., Rouard, M., Hueber, Y., Cenci, A., Hyma, K.E., Houwe, I. van den, Hribova, E., Courtois, B., Roux, N., 2016. A genome-wide association study on the seedless phenotype in banana (Musa spp.) reveals the potential of a selected panel to detect candidate genes in a vegetatively propagated crop. PLoS ONE 11, e0154448. https://doi.org/10.1371/journal.pone.0154448

Sierotzki, H., Parisi, S., Steinfeld, U., Tenzer, I., Poirey, S., Gisi, U., 2000. Mode of resistance to respiration inhibitors at the cytochrome bc1 enzyme complex of Mycosphaerella fijiensis field isolates. Pest Manag. Sci. 56, 833-841. https://doi.org/10.1002/15264998(200010)56:10<833::AID-PS200>3.0.CO;2-Q

Speijer, P.R., De Waele, D., 1997. Screening of Musa germplasm for resistance and tolerance for nematodes, INIBAP Technical Guidelines. INIBAP, Montpellier, France.

Stergiopoulos, I., Burg, H.A. van den, Ökmen, B., Beenen, H.G., Liere, S. van, Kema, G.H.J., Wit, P.J.G.M. de, 2010. Tomato Cf resistance proteins mediate recognition of cognate homologous effectors from fungi pathogenic on dicots and monocots. Proc. Natl. Acad. Sci. 107, 7610-7615. https://doi.org/10.1073/pnas.1002910107

Stergiopoulos, I., Cordovez, V., Ökmen, B., Beenen, H.G., Kema, G.H.J., de Wit, P.J.G.M., 2014. Positive selection and intragenic recombination contribute to high allelic diversity in effector genes of Mycosphaerella fijiensis, causal agent of the black leaf streak disease of banana. Mol. Plant Pathol. 15, 447-460. https://doi.org/10.1111/mpp.12104

Stierle, A.A., Upadhyay, R., Hershenhorn, J., Strobel, G.A., Molina, G., 1991. The phytotoxins of Mycosphaerella fijiensis, the causative agent of black Sigatoka disease of bananas and plantains. Experientia 47, 853-859. https://doi.org/10.1007/BF01922472

Stover R.H., 1990. Sigatoka leaf spots: Thirty years of changing control strategies: 1959-1989, in: Sigatoka leaf spot diseases of banana. International Network for the Improvement of Banana and Plantain, Montpellier, France, pp. 66-74.

Strobel, G.A., Stierle, A.A., Upadhyay, R., Hershenhorn, J., Molina, G., 1992. The phytotoxins of Mycosphaerella fijiensis, the causative agent of black Sigatoka disease, and their potenial use in screening for disease resistance, in: Biotechnology Applications for Banana and Plantain Improvement. INIBAP, Montpellier, France, pp. 93-103.

Tinzaara, W., Gold, C.S., Ssekiwoko, F., Bandyopadhyay, R., Abera, A., Eden-Green, S.J., 2006. Role of insects in the transmission of banana bacterial wilt. Afr. Crop Sci. J. 14, 105-110. https://doi.org/10.4314/acsj.v14i2.27916

Torres, J.M., Calderón, H., Rodríguez-Arango, E., Morales, J.G., Arango, R., 2012. Differential induction of pathogenesis-related proteins in banana in response to Mycosphaerella fijiensis infection. Eur. J. Plant Pathol. 133, 887-898. https://doi.org/10.1007/s10658-012-0012-7

Tripathi, L., Tripathi, J.N., Kiggundu, A., Korie, S., Shotkoski, F., Tushemereirwe, W.K., 2014. Field trial of Xanthomonas wilt disease-resistant bananas in East Africa. Nat Biotech 32, 868 870.

Upadhyay, R.K., Strobel, G.A., Coval, S.J., Clardy, J., 1990. Fijiensin, the first phytotoxin from Mycosphaerella fijiensis, the causative agent of black Sigatoka disease. Experientia 46, 982 984. https://doi.org/10.1007/BF01939396

USDA, 2008. Peru, organic product update, USDA Foreign agricultural service. USA.

Valmayor, R.V., Jamaluddin, S.H., Silayoi, B., Kusomo, S., Danh, L.D., Pascua, O.C., Espino, R.R.C., 2000. Banana cultivar names and synonims in Southeast Asia. International Network for the Improvement of Banana and Plantain-Asia and Pacific Office, Los Baños, Laguna, Philippines.

Zandjanakou-Tachin, M., Ojiambo, P.S., Vroh-Bi, I., Tenkouano, A., Gumedzoe, Y.M., Bandyopadhyay, R., 2013. Pathogenic variation of Mycosphaerella species infecting banana and plantain in Nigeria. Plant Pathol. 62, 298-308. https://doi.org/10.1111/j.13653059.2012.02650.x

Zandjanakou-Tachin, M., Vroh-Bi, I., Ojiambo, P.S., Tenkouano, A., Gumedzoe, Y.M., Bandyopadhyay, R., 2009. Identification and genetic diversity of Mycosphaerella species on 
banana and plantain in Nigeria. Plant Pathol. 58, 536-546. https://doi.org/10.1111/j.13653059.2008.01988.x

Zapater, M.F., Rakotonantoandro, A., Cohen, S., Carlier, J., 2004. Polymerase chain reactionrestriction fragment length polymorphism markers for the fungal banana pathogen Mycosphaerella fijiensis. Mol. Ecol. Notes 4, 80-82. https://doi.org/10.1046/j.14718286.2003.00575.x

Peru: Organic banana exports grow by 19\%. (2016, 22 agosto). Recuperado 16 enero, 2018, de http://www.freshplaza.com/article/162276/Peru-Organic-banana-exports-grow-by-19-procent 
CHAPTER 1 


\section{CHAPTER 2}

COMBATING A GLOBAL THREAT TO A CLONAL CROP: BANANA BLACK SIGATOKA PATHOGEN PSEUDOCERCOSPORA FIJIENSIS (SYNONYM MYCOSPHAERELLA FIJIENSIS) GENOMES REVEAL CLUES FOR DISEASE CONTROL

Arango Isaza, R.E*., Diaz-Trujillo, C*., Dhillon, B*., Aerts, A., Carlier, J., Crane, C.F., V. de Jong, T., de Vries, I., Dietrich, R., Farmer, A.D., Fortes Fereira, C., Garcia, S., Guzman, M., Hamelin, R.C., Lindquist, E.A., Mehrabi, R., Quiros, O., Schmutz, J., Shapiro, H., Reynolds, E., Scalliet, G., Souza, M., Jr., Stergiopoulos, I., Van der Lee, T.A.J., De Wit, P.J.G.M., Zapater, M.-F., Zwiers, L.-H., Grigoriev, I.V., Goodwin, S.B., Kema, G.H.J., 2016. Combating a global threat to a clonal crop: banana black Sigatoka pathogen Pseudocercospora fijiensis (Synonym Mycosphaerella fijiensis) genomes reveal clues for disease control. PLOS Genet. 12, e1005876. 


\section{Abstract}

Black Sigatoka or black leaf streak disease, caused by the Dothideomycete fungus Pseudocercospora fijiensis (previously: Mycosphaerella fijiensis), is the most significant foliar disease of banana worldwide. Due to the lack of effective host resistance, management of this disease requires frequent fungicide applications, which greatly increase the economic and environmental costs to produce banana. Weekly applications in most banana plantations lead to rapid evolution of fungicide-resistant strains within populations causing diseasecontrol failures throughout the world. Given its extremely high economic importance, two strains of $P$. fijiensis were sequenced and assembled with the aid of a new genetic linkage map. The 74-Mb genome of $P$. fijiensis is massively expanded by LTR retrotransposons, making it the largest genome within the Dothideomycetes. Melting-curve assays suggest that the genomes of two closely related members of the Sigatoka disease complex, P. eumusae and P. musae, also are expanded. Electrophoretic karyotyping and analyses of molecular markers in P. fijiensis field populations showed chromosome-length polymorphisms and high genetic diversity, but limited gene flow. Genetic differentiation was also detected using neutral markers, suggesting strong selection with limited gene flow at the studied geographic scale. Frequencies of fungicide resistance in fungicide-treated plantations were much higher than those in untreated wild-type $P$. fijiensis populations. A homologue of the Cladosporium fulvum Avr4 effector, Pfavr4, was identified in the P. fijiensis genome. Infiltration of the purified PfAVR4 protein into leaves of the resistant banana cultivar Calcutta 4 resulted in a hypersensitive-like response. This result suggests that Calcutta 4 could carry an unknown resistance gene recognizing Pfavr4. Besides adding to our understanding of the overall Dothideomycete genome structures, the $P$. fijiensis genome will aid in developing fungicide treatment schedules to combat this pathogen and in improving the efficiency of banana breeding programs. 


\section{Introduction}

Black Sigatoka or black leaf streak disease (BLSD), caused by the Dothideomycete fungus Pseudocercospora fijiensis (previously: Mycosphaerella fijiensis) (Arzanlou et al., 2008), is a major threat to global banana production (Marin et al., 2003). The disease is part of the Sigatoka complex, which involves two other closely related pathogens in addition to $P$. fijiensis: $P$. musae (previously: $M$. musicola) causal agent of yellow Sigatoka disease; and $P$. eumusae (previously: M. eumusae) causal agent of the eumusae leaf spot disease. Among the three species, $P$. fijiensis is the most aggressive and predominant member of the Sigatoka disease complex worldwide. These pathogens occur exclusively on the foliage of bananas and plantains, with continuous sexual and asexual reproduction in nature (Arzanlou et al., 2008; Carlier et al., 2000, 1996; Crous and Mourichon, 2002).

BLSD was first reported in the Sigatoka Valley of the Fiji islands during the 1960s and has since spread to nearly all banana-producing areas worldwide. It can only be managed by intensive fungicide applications, requiring weekly interventions throughout the year in most production areas. Black Sigatoka inflicts huge costs on global banana production, surpassing US \$500 million per year (Ploetz, 2004). Expenses for fungicide treatments usually represent more than $35 \%$ of total production costs (de Bellaire et al., 2010; Romero and Sutton, 1997). Infection with P. fijiensis also results in crop losses and massive indirect costs by inducing early ripening of the fruit, making it unsuitable for sale with concomitant effects on the export trade and the retail sector.

Export banana cultivars are sterile, triploid plants that can only be propagated clonally and are grown in huge monocultures of genetically identical individuals. The international banana trade is based solely on a few closely related clones of the Cavendish type, all of which are highly susceptible (Ploetz, 2004); disease management, therefore, relies primarily on fungicide applications with enormous environmental impacts (Churchill, 2011). Moreover, 
the selection pressure on $P$. fijiensis populations continuously reduces the efficacy of fungicides resulting in control failures and unmanageable levels of disease (Marin et al., 2003; Romero and Sutton, 1997). Therefore, there is an urgent need for scientific discoveries that will lead to the development of better methods for protecting banana crops, both for export fruit production and for small holders around the world who rely on bananas as a staple food (Marin et al., 2003).

Taxonomically, Pseudocercospora belongs to the order Capnodiales in the class Dothideomycetes, previously known as the Loculoascomycetes (Eriksson and Winka, n.d.), which is the largest and most diverse class of ascomycete fungi comprising over 20,000 species. Dothideomycete fungi include endophytes and epiphytes of plants, but also saprobes degrading cellulose and other complex carbohydrates of dead plants, and plant pathogens (Schoch et al., 2006). The latter cause a range of diseases in various key food, fiber and fuel crops, including Zymoseptoria tritici (Septoria tritici blotch of wheat) (Kema, 1996), Venturia inaequalis (apple scab) (Bowen et al., 2011), and Leptosphaeria maculans (blackleg of Brassica crops) (Howlett et al., 2001). Therefore, genome sequences of several Dothideomycetes have been published (Ellwood et al., 2010; Hane et al., 2007; Goodwin et al., 2011; de Wit et al., 2012; Ohm et al., 2012; Condon et al., 2013; Manning et al., 2013; Dhillon et al., 2015) or are in the process of being completed (http://genome.jgi.doe.gov/dothideomycetes/dothideomycetes.info.html). The genome sequence of Z. tritici is the reference for all other Dothideomycetes as it is the only one that has been completely finished (Manning et al., 2013).

The poor experimental amenability of $P$. fijiensis has significantly hampered progress in understanding its basic biology (Churchill, 2011) and the development of research tools. For instance, infection assays are cumbersome due to the need for very specific environmental conditions with respect to temperature, light and relative humidity, and the slow development 
of the disease that may take up to 50 days until symptoms are expressed (Abadie et al., 2008; Nfor et al., 2011). Therefore, basic information on pathogenesis is not available and almost nothing is known about the genetic basis of disease resistance in banana germplasm (Beveraggi et al., 1993; Torres et al., 2012). Hence, new tools and research methods are needed to better understand the disease and ensure continued production of the world's number one fruit, which is a staple food for millions of people in many developing countries.

A previous comparative analysis of 18 Dothideomycetes genomes (Ohm et al., 2012) included the $P$. fijiensis isolate CIRAD86 genome for a global analysis of genome organization and evolution. However, P. fijiensis was not the primary focus of that analysis and few specifics were discussed. Here we focus on the genome sequence of $P$. fijiensis isolate CIRAD86, describe the sequence of a second isolate, CIRAD139, and analyze in detail the species' genome structure, content and function with a goal of delivering new data that could give clues for global disease management of this devastating banana pathogen.

\section{Results}

\section{Sequencing, assembly and annotation of the $P$. fijiensis genome}

The genomes of the $P$. fijiensis isolates were sequenced using either Sanger technology (CIRAD86) or Illumina for resequencing (CIRAD139a). The final assembly size of $\sim 74 \mathrm{Mb}$ consisted of 56 scaffolds with the largest at $11.8 \mathrm{Mb}$ and an N50 of $50 \mathrm{~Kb}$. Inclusion of a newly made genetic map facilitated assembly of the physical genome (Table S1).

Genetic map construction involved 376 loci that segregated in the progeny of the mapping population, among which 322 (233 DArT, 86 SSR, 3 minisatellite) markers were mapped into 19 linkage groups (Figure 1). The number of loci per linkage group varied from 
2 to 35 with an average of 17 and linkage groups $1,2,8$ and 9 contained the largest numbers of markers with 35, 29, 31 and 26, respectively. Map distances between consecutive markers varied from 0 to $20.4 \mathrm{cM}$ with the largest gaps between markers on linkage groups 14 and 17, of 6.1 and $20.4 \mathrm{cM}$, respectively (Figure 1).

After filtering for EST support, completeness and similarity to other species, 13,107 genes were structurally and functionally annotated. The average gene length in the version 2 assembly is $1,833 \mathrm{nt}$ with 3.62 exons per gene; $88 \%$, are complete with start and stop codon, $74 \%$ have similarity support, and $49 \%$ have Pfam domains (Table S2). Most of the gene models $(96 \%)$ are located in 12 scaffolds, numbers 1-10, 12 and 19. Gene density in these 12 scaffolds varies from 151 to 229 per Mb but gene density for the remaining scaffolds larger than $0.5 \mathrm{Mb}$ drops to only 2 to 94 genes per Mb (Table S1). More detailed information on the assembly, annotation and EST support data can be found in supporting Text S1.

\section{Genome structure}

The Pseudocercospora fijiensis genome is greatly expanded

The 74-megabase genome of P. fijiensis is greatly expanded relative to those of other related Capnodiales such as Sphaerulina musiva (previously Septoria musiva with teleomorph Mycosphaerella populorum), S. populicola (previously Septoria populicola with teleomorph Mycosphaerella populicola) and less related species such as Dothiostroma septosporum, Baudoinia compniacensis, and Z. tritici, but less so compared to C. fulvum, the closest Capnodiales relative sequenced and only other Dothideomycete with an expanded genome of $65 \mathrm{Mb}$ (Figure 2). The predominant repetitive elements in the P. fijiensis genome belong to the long terminal repeat (LTR) retrotransposons (50\%) (Figure 3), which is much higher than in Z. tritici, but similar to the proportion seen in C. fulvum. Compared to these other two 
species, the genome of $P$. fijiensis contained much higher percentages of repetitive DNA and unclassified transposons, whereas that of $C$. fulvum had the highest percentage of non-LTR retrotransposons among the three species (Figure 3). The estimated number of gene models is 13,107, which is approximately $28 \%$ and $34 \%$ higher than in S. musiva and S. populicola, respectively (Table 1) and 7\% smaller than $C$. fulvum, Using the 80:80 criterion (Wicker et al., 2007), i.e., $80 \%$ sequence identity across $80 \%$ alignment length, all of the $P$. fijiensis repeat families were unique. However, using a 70:70 cutoff criterion, elements from $50 P$. fijiensis repeat families, amounting to $449 \mathrm{~kb}$, were similar to those in the C. fulvum genome. A non-LTR repeat family from P. fijiensis (family 6), with an average element length of 4.9 $\mathrm{kb}$, had the highest representation with 36 copies in the $C$. fulvum genome.

Analysis of repeat-induced point mutation (RIP) showed a clear CA<-> TA dinucleotide bias in the repetitive elements identified in the $P$. fijiensis genome (Figure 4 ). Some families also showed a $\mathrm{CT}<->\mathrm{TT}$ dinucleotide bias. A similar pattern has been observed in a number of ascomycete genomes, including Parastagonospora nodorum (Hane and Oliver, 2008).

Repetitive elements often clustered to form blocks of AT-rich DNA. When an average DNA content of $45 \%$ or less was used to define AT-rich regions, a total of 1,865 AT blocks was identified in the $P$. fijiensis genome, ranging in length from 1 to $514 \mathrm{~kb}$. These blocks account for $45 \mathrm{Mb}(61 \%)$ of the $P$. fijiensis genome and $84 \%$ comprised repetitive sequences. A total of $482(4 \%)$ genes were associated with the AT blocks. About 20\% (96) of these genes have associated annotations and 6\% (28) can potentially be secreted.

If a lower value of $40 \%$ average percent $\mathrm{GC}$ is used, the number of AT blocks diminished drastically to 640 , amounting to a total length of $18 \mathrm{Mb}$ or approximately $25 \%$ of the $P$. fijiensis genome. Repetitive sequences make up 84\% and $152(1.2 \%)$ genes were 
associated with these AT-rich blocks. Approximately 22\% (33) of the genes associated with AT blocks have an annotation and about 10\% (15) have signal peptides.

The average RIP index was 0.2 in the AT-rich blocks as compared to a higher average RIP index of 1.37 across the rest of the genome. Plots of the RIP index were very low (indicating a high level of RIP) in the AT blocks (Figure 5A) but much higher (low RIP) in the regions of the genome with lower AT content (Figure 5B). As expected, there was a strong inverse relationship between GC content and the amount of RIP as measured by the index (Figure S1). Very few of the genes (just over 3\%) in AT-poor (= GC rich) regions of the genome showed any evidence or RIP (index of 1.0 or less) compared to a little over half (53\%) of those in AT-rich regions (Table 2). In contrast, all but two out of 7,674 repeats in AT-rich regions showed evidence of RIP and almost 93\% of those in AT-poor regions (Table 2). Exceptions were few and minor (Figure S2).

First-derivative graphs obtained for melting profiles of $Z$. tritici showed a narrow curve with a single peak (Figure 6A); in contrast, those for P. fijiensis showed a broad curve with two peaks with $\mathrm{G}+\mathrm{C}$ contents of 39.4 and $51.6 \%$ indicating heterogeneity (Table S3). This agreed with the GC plots of sequence reads from $P$. fijiensis, which clearly showed a double-peak phenomenon, the lower peak corresponding to transposon-rich regions (Figure $6 \mathrm{E})$.

The melting profiles obtained from the DNA of both P. eumusae and P. musae also demonstrated a double-peak pattern of genomic $\mathrm{G}+\mathrm{C}$ content. The $\mathrm{G}+\mathrm{C}$ content pattern in $P$. eumusae was almost identical to that in P. fijiensis with peaks at 39.6 and 51.6\%. In P. musae, both peaks corresponded to lower, albeit still comparable, $\mathrm{G}+\mathrm{C}$ contents of $37.2 \%$ and $50.9 \%$ (Figure 6 A-D, Table S3). Plotting genome size on a phylogenetic tree of the Capnodiales 
identified at least two expansions, one leading to $P$. fijiensis and the second to the biotrophic tomato pathogen C. fulvum (Figure 2).

To estimate the age of the transposon expansion in the $P$. fijiensis genome, approximately1600 LTR retrotransposons were identified using ltrharvest. A further search for protein domains identified 1,147 bona fide, full-length LTR retrotransposons. Of these, 529 elements (46\% of the total) had LTRs that were highly similar in terms of mutations accumulated over time with a hypothetical insertion age of less than one million years (Figure 7). Many older elements also were identified (Figure 7) but these decreased with time, indicating that most of the transposon insertions occurred relatively recently.

Electrophoretic karyotyping suggests variability in genome content and/or organization among isolates

Pulsed-field gel electrophoresis of the CIRAD86 and E22 strains showed small and large chromosomes, but no chromosomes in the medium range of 1.5 to $3.9 \mathrm{Mb}$. Isolate CIRAD86 showed 11 bands representing chromosomes, four of which appeared to be composed of double, co-migrating bands (Figure 8A). Small chromosomes were in the size range of 0.83 to $1.45 \mathrm{Mb}$. Bands of 0.95 and $1.03 \mathrm{Mb}$ showed approximately twice the intensity and were assumed to represent at least two chromosomes each (Figure 8A). Conditions for separation of large chromosomes showed a band of $5.2 \mathrm{Mb}$, a co-migrating chromosomal band of 4.33 and a smaller band of $4.27 \mathrm{Mb}$ (Figure 8B). Strain E22 showed at least 12 bands in total, five of which likely contain co-migrating chromosomes. Small chromosomes were in the range of $0.70-1.45 \mathrm{Mb}$, and large chromosomes had estimated lengths between 4.05 and $6.80 \mathrm{Mb}$ (Figure 8B). Additionally a comparison of small chromosomal bands of different strains originating from a single banana field showed that 
every isolate contained between five and nine small chromosomal bands with unique length polymorphisms (Figure S3) indicating substantial variation in genome content and/or genome organization among individuals.

Synteny analysis suggests a core set of 12 chromosomes

Analysis of similarity between the genomes of different Dothideomycetes shows a high degree of conservation of genes in syntenic scaffolds. Mesosynteny was observed between P. fijiensis and all other Dothideomycetes analyzed including the Capnodiales $B$. compniacensis, Cercospora zeae-maydis, Cladospoerium fulvum, D. septosporum (Figure 9), S. musiva (Figure S4), Zasmidium cellare and Z. tritici, the Pleosporales Cochliobolus heterostrophus, L. maculans, Pyrenophora tritici-repentis and P. nodorum, and the Hysteriales species Hysterium pulicare. Microsyntenic blocks of up to $10 \mathrm{~Kb}$ were found only with the closest relatives C. fulvum, D. septosporum and Z. tritici (S. musiva was not tested for this analysis). No macrosynteny was observed between $P$. fijiensis and any of the presently sequenced Dothideomycetes.

Using Z. tritici as a reference it is clear that gene content is conserved among large blocks of chromosomes. For example, scaffold 1 of $P$. fijiensis shows synteny with chromosomes 1, 4 and 5 of Z. tritici, scaffold 2 with chromosomes 2, 10 and 13, whereas scaffold 6 of $P$. fijiensis shows synteny only with Z. tritici chromosome 6 (Figure S5). Interestingly, no significant synteny was found between any of the scaffolds of $P$. fijiensis and the dispensable chromosomes of Z. tritici (Figure 10), supporting the hypothesis of their independent origin, possibly by recent horizontal transfer, in the latter species (Goodwin et al., 2011).

Most of the synteny found in P. fijiensis with Z. tritici as well as with all other Dothideomycetes tested is present in scaffolds 1 through 10, 12 and 19. In addition, these 
scaffolds showed the highest percent of genes with expression data ( $0.8 \%$ or greater), all of which suggest that these 12 scaffolds might represent the core genome. Many of the smaller scaffolds in the $P$. fijiensis genome have the physical characteristics observed for dispensable chromosomes in Z. tritici; they are smaller, with lower $\mathrm{G}+\mathrm{C}$ contents and gene densities (Table S1). Based on these criteria, the core genome of $P$. fijiensis comprises $63.9 \mathrm{Mb}$ or almost $87 \%$ of the genome, while the remaining $13 \%$ may be a dispensome.

Analysis of the synteny plots also showed some past chromosomal rearrangements. For example, approximately $22 \%$ of the gene content of the central part of scaffold 1 of $P$. fijiensis was missing from the largest scaffolds of D. septosporum (Figure 9) and S. musiva (Figure S4), and instead was found on scaffolds 8 and 7 of those species, respectively. This difference also was seen in the comparison with the more distantly related Z. tritici (Figure S5), although the result was not as clear and more chromosomes were involved. In a direct comparison, scaffold 1 of $D$. septosporum showed complete mesosynteny with scaffold 1 of S. musiva (Figure S6), suggesting that the central part of $P$. fijiensis scaffold 1 might have translocated after the divergence of all three species from an unknown common ancestor. The chromosome that likely supplied the translocation, corresponding to scaffolds 8 and 7 in $D$. septosporum and S. musiva, respectively, showed 1:1 mesosynteny in the direct comparison between those two species (Figure S6), but also showed mesosynteny with P. fijiensis scaffolds 12 and 19 (Figures 9 and S4). This suggests that scaffolds 12 and 19 of P. fijiensis may belong to a single chromosome that has not been assembled completely. Similar analyses identified a possible translocation or incomplete assembly involving scaffolds 3 and 8 of $P$. fijiensis, which correspond to scaffolds 5 (Figure 9) or 4 (Figure S4) of D. septosporum and S. musiva, respectively. 
Re-sequencing of $\mathrm{P}$. fijiensis isolate CIRAD139A shows a $12 \%$ difference in genome content

Among the more than 73 million reads of paired-end sequence data obtained for isolate CIRAD139A, 60\% could be aligned uniquely to the $P$. fijiensis reference genome of isolate CIRAD86 (Table S4). Another $28 \%$ of the reads aligned to multiple locations in the reference sequence, most likely due to duplications or repetitive elements in the genome. Almost $12 \%$ of the reads did not map to the reference, suggesting that some genome content present in CIRAD139A could be absent in the reference strain.

The numbers of polymorphisms varied widely among scaffolds roughly in proportion to size, and the number of SNPs was much higher than for indels on all scaffolds analyzed (Table S5). Mean SNP frequency on each scaffold calculated across a 10-kb window was more uniform, ranging from 59.2 for scaffold 13 to 84.1 for scaffold 11 (Table S5). Plotting the SNP density relative to gene density for the 21 largest scaffolds containing $99 \%$ of all gene models separated the scaffolds into two groups. One group contained most of the largest scaffolds and showed lower variability in SNP counts, while the second contained scaffolds with low gene densities and showed much more variability in SNP counts.

\section{Genome content}

\section{Decreased numbers of pathogenicity-related genes}

Enzymes that degrade plant cell walls (CWDEs) and other physical barriers including cutin are important pathogenicity factors, particularly in necrotrophic fungi. Comparison of the number of genes related to cell wall degradation in P. fijiensis with those in other fungi revealed a significant reduction, particularly when compared to necrotrophic Dothideomycetes. Cutinases, xylanases and chitinases are reduced three to five-fold when 
compared to three fungi in the Pleosporales: $P$. nodorum, $P$. tritici-repentis and $C$. heterostrophus (Table 3). Additionally, carbohydrate-binding proteins, including those with chitin-binding and cellulose-binding modules as well as $\beta$ glucosidases also are reduced (Table 3). EST support was found for four chitinases, 18 glucosidases, one cellulose binding and four chitin binding genes.

Similar to the CWDEs, the $P$. fijiensis genome shows a relatively low number of genes involved in the production of secondary metabolites, such as polyketide synthases (PKSs), with approximately half the number of genes found in the necrotrophs $P$. nodorum, $P$. triticirepentis and $C$. heterostrophus. Contrary to the situation for PKSs, genes encoding nonribosomal peptide synthetases (NRPSs) are not reduced in P. fijiensis. Its genome encodes 13 NRPSs and one hybrid NRPS-PKS, which is comparable to the numbers found in other Dothideomycetes (Table 3). However, EST support was found for only six of the PKS genes and four of the NRPS and the hybrid NRPS-PKS genes. This low level of EST support might be a sampling phenomenon due to the EST coverage; none of the libraries came from in planta conditions where these genes are more likely to be expressed.

The P. fijiensis secretome

Filamentous fungal pathogens are able to modulate resistance responses in the plant cell by secreting a class of proteins known as effectors. In many fungal pathosystems, effectors are important pathogenicity or virulence factors that determine the success of a fungal infection (de Wit et al., 2009; Oliva et al., 2010). The majority of described fungal effectors share many characteristics and belong to the class of small, secreted, cysteine-rich proteins (SSPs) (de Wit et al., 2009). A search of the genome with the above criteria showed that $P$. fijiensis possesses 172 genes encoding SSPs (smaller than 300 AAs in size) with four or more cysteine residues. Sixty-two percent of the P. fijiensis SSPs have no blast hits (107 
proteins), $21 \%$ (37 proteins) have assigned GO terms and $23 \%$ have InterPro IDs other than SignalP (40 proteins). Thus, the number of potential SSP-encoding genes in P. fijiensis is $31 \%$ and $8 \%$ lower than in the genomes of P. nodorum (250 genes) and Z. tritici (187 genes), respectively (Figure S7). These results accord with Ohm et al. 2012 who found reduced numbers of SSPs in several Capnodiales.

Among the identified SSPs, one shows high similarity to C. fulvum Avr4, which is known to have a chitin-binding domain and is a well-studied effector in the C. fulvum-tomato interaction (van Esse et al., 2007). This P. fijiensis putative Avr4 (PfAvr4) homolog is a 121 amino acid protein present on scaffold 4 from co-ordinates $183261-183623$ and was shown to protect Trichoderma viride cell walls against hydrolysis by plant chitinases through chitin binding and to trigger a $C f 4$-mediated hypersensitive response (HR) in tomato (Stergiopoulos et al., 2014, 2010). Additionally, three homologs of C. fulvum effector Ecp2 were found, one of which was able to induce different levels of necrosis or HR in tomato lines depending on whether they lack or contain a putative corresponding Cf-ECP2 protein (Stergiopoulos et al., 2014, 2010). It seems highly likely that at least some of these $P$. fijiensis effector proteins that are similar to known effectors in $C$. fulvum will play a role in pathogenicity or virulence of $P$. fijiensis on banana.

\section{Genome function}

\section{Functional analysis of a putative effector protein}

Infiltrations into banana and tomato leaves were performed to test the hypothesis that PfAvr4 acts as an avirulence factor in banana. Different accessions were infiltrated with different concentrations of PfAVR4. Physical damage (small tear and occasional slight necrosis limited to the site of infiltration) caused by either the syringe or the fermentor 
medium was similar among accessions (Figure 11) and was less intense in tomato plants (Figure 11B). Both large and small plants of M. acuminata cv. Grand Naine showed only physical damage with an occasional slight chlorotic effect at the infiltrated area (Figure 11A) after infiltrating the PfAVR4 protein; no hypersensitive response (HR) was observed at 10 days post infiltration (dpi) regardless of the protein concentration used.

In contrast, PfAVR4 triggered a clear HR-like necrosis when infiltrated into leaves of M. acuminata ssp. burmannicoides var. Calcutta IV, which has resistance against $P$. fijiensis (Figure 11A). The necrosis was already visible on large plants at 4 dpi, and was stronger by 10 dpi at both concentrations of PfAVR4. In small plants the earliest necrosis was observed at 10 dpi. Fermentor medium triggered a slight necrosis on both small and large plants of var. Calcutta IV, but this was very different from the HR-like necrosis induced by PfAVR4 and the combined effect triggered by the fermentor product (Figure 11A). Furthermore, tomato plants without a resistance gene ( $C f O$ plants) showed only physical damage following infiltration, while those containing the $C f 4$ resistance gene showed a HR to crude fermentor products containing PfAVR4 and to the purified PfAVR4 protein (Figure 11B).

\section{Analyses of fungicide resistance and molecular markers within populations}

In total, 621 hierarchically sampled $P$. fijiensis isolates were genotyped and partially phenotyped and showed that the commercial (sprayed) plantations were entirely or nearly fixed for quinone outside inhibitor (QoI) or strobilurin resistance (92-100\%), whereas all 87 isolates sampled from the wild type, unsprayed San Carlos population were sensitive (Table S6). Subsequently, we used the genome sequence to develop primers for five Variable Number of Tandem Repeat (VNTR) loci enabling population diversity analyses that were combined with assessment of the mating type loci. We observed that the ratios between the two mating type alleles mat1-1 and mat1-2 are not significantly different from 1:1 in each 
individual population as well as the overall total number of isolates (Table S6), and that the VNTR loci in all populations are in gametic equilibrium and hence, could be used to estimate genetic differentiation between populations, which was small but statistically significant (Table S7). 


\section{Discussion}

The genome of $P$. fijiensis is one of the largest among all of the Dothideomycetes sequenced to date; it is 3.4 times larger than that of the Dothideomycete with the smallest genome, Baudoinia compniacensis, 1.85 times larger than that of $Z$. tritici and 1.2 times larger than that of $C$. fulvum, which is related to P. fijiensis and also has an expanded genome (de Wit et al., 2012). Almost all of the increased size is due to the proliferation of LTR retrotransposons, as described here and in another publication (Santana et al., 2012). The thermal denaturation results indicate that $\mathrm{G}+\mathrm{C}$ content heterogeneity is not limited to $P$. fijiensis but also occurs in its close relatives within a monophyletic clade of banana pathogens. Based upon the observed similarity of DNA composition between these three banana pathogens, we predict that the genomes of both $P$. eumusae and $P$. musae also are expanded and that all three pathogens that often co-occur in nature seem to have a recent common ancestor (Carlier et al., 2000).

The only other member of the Capnodiales known to have a similarly expanded genome is the biotrophic tomato pathogen, C. fulvum (de Wit et al., 2012). Based on the positions of the species with expanded genomes on the phylogenetic tree (Figure 2), there appear to have been at least two independent expansions in genome size within the Capnodiales, one involving P. fijiensis (and most likely related banana pathogens) and another for C. fulvum. Lack of similarity between the transposable elements in the genomes of P. fijiensis and C. fulvum supports the hypothesis of independent expansions in each genome.

The terminal repeats of LTR retrotransposons are identical at the time of insertion and this provides a means to estimate the relative ages of transposable element insertions. This phenomenon has been very useful for estimating transposon insertion times in plants (SanMiguel et al., 1998; Wicker and Keller, 2007) but is less useful for fungi where RIP 
greatly increases the rate of mutations in repetitive sequences. Using this approach in the basidiomycete Laccaria bicolor, three periods of transposon insertion were identified, ranging in age from 0 to 59 million years ago (Mya) (Labbé et al., 2012). Whether RIP occurs in $L$. bicolor is not known, but its genome does not contain the genes known to be required for RIP in other fungi (Smith et al., 2006) and no evidence for RIP was found among transposons in the genomes of other members of the Agaricomycotina (Horns et al., 2012) so it seems unlikely. Therefore, this approach for estimating the ages of transposon insertions is most likely valid for L. bicolor.

A similar analysis of LTRs of transposons in the genome of Z. tritici yielded hypothetical insertion ages ranging from 0 to $5.7 \mathrm{Mya}$, with the strong caveat that the times were probably vastly overestimated due to RIP (Dhillon et al., 2014). Even with the bias introduced by RIP, the estimated ages of transposon insertions in the genome of $Z$. tritici were an order of magnitude younger than those in L. bicolor, indicating that they must have occurred relatively recently. A more accurate approach to estimating transposon age in the presence of RIP would be to exclude the RIP-susceptible sites from analysis (Rouxel et al., 2011). Using this approach, transposon insertions in L. maculans and closely related species in the Pleosporales, another large order of the Dothideomycetes, mostly were relatively recent, within the past four million years (Rouxel et al., 2011). For $P$. fijiensis, the results of a similar analysis clearly suggest a recent, rapid burst of LTR retrotransposon insertions. The young age and high proliferation rate of around $46 \%$ of the LTR retrotransposons suggest that P. fijiensis has a highly dynamic genome. Such a recent, high level of activity of retrotransposons can have evolutionary as well as regulatory implications for gene expression that can be better understood using genomic comparisons with other closely related species.

Large genome expansions due to amplifications of repetitive elements have been observed in other plant-pathogenic fungi. The published genome sequences and analyses of 
the powdery mildew fungi Blumeria graminis f. sp. hordei, Erysiphe pisi and Golovinomyces orontii show a marked genome expansion with a massive proliferation of non-LTR retrotransposons and a corresponding decrease in gene content (Spanu et al., 2010). The missing genes in these obligate biotrophs include enzymes for primary and secondary metabolism, carbohydrate-active enzymes, transporters, and secreted proteins such as effectors. The genome of $P$. fijiensis also shows a moderate decrease in certain gene families associated with pathogenicity such as PKSs and CWDEs when compared to necrotrophic Dothideomycetes such as $P$. nodorum or P. tritici-repentis (Hane et al., 2007). In a similar way, the hemibiotrophs Z. tritici (Goodwin et al., 2011), S. populorum and S. populicola (Dhillon et al., 2015; Ohm et al., 2012) also show a marked decrease in CWDEs [19], although not to the extent seen in the powdery mildews. This reduction in Z. tritici is thought to have evolved as a mechanism to evade detection by host defenses during stealth pathogenesis (Goodwin et al., 2011). This hypothesis also could fit the lifestyle of P. fijiensis since it has an even longer biotrophic phase of up to 28 days before necrotic symptoms start to appear so may have a greater need for stealth (Nfor et al., 2011). However, the association is not perfect because the reduction in CWDEs in Z. tritici is greater, particularly for $\beta$ glucosidases.

Thus, within Ascomycetes there seems to be a correlation between pathogenic lifestyle (biotrophic vs. hemibiotrophic vs. necrotrophic) and diversity of certain gene families such as PKSs and CWDEs. This correlation does not hold up when extended to other fungal groups. For example, rust fungi are obligate biotrophs with greatly expanded genomes due to either the proliferation of LTR retrotransposons similar to $P$. fijiensis for the wheat stem rust pathogen Puccinia graminis, or to class II DNA transposons for the poplar rust fungus Melampsora larici-populina (Duplessis et al., 2011). However, in both rusts gene numbers were greatly expanded including those for SSPs that may be involved in interactions with 
their hosts (Duplessis et al., 2011). Thus, evolution of a biotrophic lifestyle has involved very different changes in the genetic architecture of fungal genomes, from the vast reductions in gene content noted in the powdery mildew fungi to huge expansions for the rusts. Hemibiotrophs such as $P$. fijiensis fall in the middle of the continuum. The one constant is the increased genome size due to transposons, which seems much more common in biotrophic and hemobiotrophic pathogens compared to necrotrophs or saprotrophs.

The mechanisms of these transposon expansions remain mostly unknown. The two main unanswered questions revolve around the source of the invading elements and the mechanism by which they proliferate. The most obvious source would be their host plants, but so far there appears to be little evidence that transposons are being transferred from hosts to their pathogens. Biotrophic fungi should be the most suited for acquiring transposons because they are restricted to growing in a very limited ecological niche and have specialized feeding structures to retrieve nutrients from their hosts. For $P$. fijiensis, a search of the banana genome sequence revealed that transposable elements account for almost half of the Musa sequence with LTR retrotransposons representing the largest part (Arango et al., 2011; D’Hont et al., 2012), so the transposons might have come from banana. If not from the hosts, then they most likely have been acquired from other fungi or pests that are associated with the hosts. Horizontal transfer of genes has been shown in other fungi such as P. nodorum (Friesen et al., 2006) and Fusarium oxysporum f. sp. lycopersici (Ma et al., 2010) and it could occur for transposons. Horizontal transfer has the potential to broaden host range and pathogenicity of fungal pathogens or even create a new pathogen from a non/pathogenic strain (Friesen et al., 2006; Ma et al., 2010). Solving the mystery about the origin of invading transposons is important for understanding the dynamics of fungal genome expansions, and the causal agents of the Sigatoka complex on bananas represent a good model to address such a question. 
A little more is known about the mechanisms for transposon expansion after they have been acquired. Almost all fungi are capable of Repeat-Induced Point (RIP) mutation, a mechanism for identifying and mutating repetitive sequences (Cambareri et al., 1989; Goyon and Faugeron, 1989; Grayburn and Selker, 1989; Rouxel et al., 2011; Selker et al., 1987). For transposons, the mutations caused by RIP prevent successful translation of the genes coding for transposon movement proteins so they become inactive and can no longer replicate. This provides a very effective defense against transposon expansions in most fungi. In the powdery mildew fungi, the genetic machinery required for RIP was missing (Spanu et al., 2010) and this likely allows unrestricted multiplication of transposons. For the rust fungi, no mechanisms for genome expansion were proposed or tested (Duplessis et al., 2011).

For P. fijiensis, rid, the only gene known to be required for RIP, is present and the reading frame appears to be intact. Repetitive sequences in the genome of $P$. fijiensis show high frequencies of the $\mathrm{C}$ to $\mathrm{T}$ transitions that are characteristic of RIP, so this phenomenon seems to be active. Because RIP is only active during meiosis, a possible explanation for fungi with extensive asexual phases could be that transposon expansion occurs during asexual reproduction and then is slowed by RIP during rare sexual reproduction. If transposons have expanded enough and RIP is not completely efficient, some intact copies of transposons could remain after meiosis to continue expanding during the next extended asexual phase. This explanation is possible in $P$. fijiensis; although it has been classically considered to be primarily reproducing by sexual mating, more recent studies suggest that asexual reproduction also plays an important role during epidemics (Rieux et al., 2013a, 2014). Transposon expansion most likely occurs episodically when RIP or other mechanisms are relaxed, but when and how these episodes occur is currently unknown.

In Z. tritici, a different type of genome expansion occurred through the acquisition of a large set of dispensable chromosomes, referred to as the dispensome (Goodwin et al., 2011). 
The Z. tritici dispensome contains at least eight chromosomes with no known function that appear to have been acquired by horizontal transfer from an unknown donor more than 10,000 years ago (Stukenbrock et al., 2010). Such a large number of dispensable chromosomes with no known effects on fitness so far is unique among fungi. Many potential dispensable chromosomes were identified among the genomic scaffolds of $P$. fijiensis based on the characteristics of known dispensable chromosomes in Z. tritici. However, dispensability still has not been proven for $P$. fijiensis. None of the $P$. fijiensis linkage groups were missing in progeny isolates, one of the hallmarks of dispensable chromosomes in Z. tritici (Wittenberg et al., 2009). However, this is not surprising because none of the linkage groups corresponded to any of the putative dispensable scaffolds. If $P$. fijiensis does contain a dispensome, it is different from that in Z. tritici because there was almost no similarity between the dispensable chromosomes of $Z$. tritici and any of the scaffolds of $P$. fijiensis, or vice versa. This raises the intriguing possibility of separate events leading to horizontal transfer of large numbers of chromosomes between species in the Capnodiales and other fungi.

Electrophoretic karyotyping of $P$. fijiensis showed a remarkable level of variability among isolates, even those coming from the same population. This high variability in chromosome length and number was also described previously in P. fijiensis (RodríguezGarcía et al., 2006) and in other fungi (Cooley and Caten, 1991; Iwaguchi et al., 1990; Talbot et al., 1993). The mechanisms of such variation include chromosome rearrangements during meiotic recombination and the presence of dispensable chromosomes (Zolan, 1995). From a different perspective, it has been shown that chromosomal reshuffling can drive evolution of virulence in asexual plant-pathogenic fungi (Jonge et al., 2013); thus both sexual and asexual life cycles could be a source of chromosomal variation. This could constitute a mechanism of adaptation to environmental changes such as selective pressure from chemical fungicides. 
Field isolates karyotyped in this work were collected in Costa Rica in an area with a high level of fungicide applications.

Overall, P. fijiensis chromosomes are larger than those from other Ascomycetes, including its completely sequenced relative Z. tritici (Goodwin et al., 2011). Remarkably, P. fijiensis contains a scaffold larger than $10 \mathrm{Mb}$, which is at the limit of PFGE resolution. Chromosomes of this size have been observed in other fungi (Cox et al., 1990; Migheli et al., 1995; Orbach et al., 1988) but they are not common. Medium-sized chromosomes were not found, similar to previous records for Mexican isolates (Rodríguez-García et al., 2006). Interestingly, the seven smallest main scaffolds, including the smallest calculated core chromosome $(0.61 \mathrm{Mb})$ and six of the putative dispensable chromosomes did not appear in PFGE, as CIRAD86 did not show a chromosome smaller than $0.8 \mathrm{Mb}$. The total number of chromosomes separated by PFGE is at least 11 , and probably up to 15 when possible comigrating bands are counted separately, in addition to the five unresolved largest scaffolds.

The availability of a genome sequence enables the identification of genes that might be involved in pathogenicity, including those encoding putative effector proteins. Fungal effectors are proteins that aid pathogenicity, usually by subduing host defenses. However, these same proteins affecting pathogenicity also can be recognized by the host resistance proteins, triggering a defense response and making them advantageous or disadvantageous to the pathogen depending on the host genotype. Bioinformatic analysis of the P. fijiensis genome identified many putative effectors, including one that appears to be a homolog of the Avr4 effector in the related Dothideomycete, $C$. fulvum (van Esse et al., 2007). The P. fijiensis putative Avr4 homolog Pfavr4 was on scaffold 4 adjacent to repeats of 617 and $2765 \mathrm{bp}$ and a 6-kb AT-rich block with a GC content of 39.3\%, similar to known effectors in other fungi, which often are in AT-rich regions (Rouxel et al., 2011; Stukenbrock et al., 2010). Other genes in this region of the $P$. fijiensis genome were different from those in Z. tritici and $C$. 
fulvum, indicating little synteny among genes around the Pfavr4 homologs in related species. Previous research showed that PfAVR4 is a functional homolog of the CfAVR4 virulence factor in C. fulvum, and that, despite a low amino acid identity of only $42 \%$, it could be recognized by the Cf4 resistance protein to stimulate an HR in tomato (Stergiopoulos et al., 2010). However, whether PfAvr4 could be recognized by banana cultivars had not been tested until now.

The response of the banana varieties to the PfAVR4 protein strongly suggests that it acts as an avirulence factor that is recognized by a resistant banana accession where it elicits an HR-like necrosis. Most probably this protein has a function similar to that of its homolog in $C$. fulvum where it is an effector that facilitates disease in susceptible cultivars of tomato and can be recognized by the Cf- 4 receptor in a resistant tomato cultivar to elicit the HR (van Esse et al., 2007). To our knowledge, this is the first fungal effector known to induce a cultivar-specific, HR-like necrosis in banana, suggesting that $M$. acuminata ssp. burmannicoides var. Calcutta IV most likely has a functional $\mathrm{R}$ gene that recognizes PFAVR4, and which appears to be a functional homologue of Cf4 in tomato. Additional experiments are needed to thoroughly test the hypothesis that var. Calcutta IV contains an HR-inducing resistance gene effective against $P$. fijiensis. These could include analysis of progeny from controlled crosses between var. Calcutta IV and a susceptible banana to test for co-segregation of necrosis induced by PfAVR4 and resistance to P. fijiensis, or deletion of PfAvr4 to test whether resulting mutant becomes virulent to var. Calcutta IV. However, these experiments would be challenging due to experimental limitations in this pathosystem: crosses in banana frequently suffer from segregation distortions due to the occurrence of translocations and functional analyses in P. fijiensis are not routine.

The banana var. Calcutta IV has been a source of resistance against fungi, bacteria and nematodes in Musa breeding programs (Ortiz and Vuylsteke, 1994; Simmonds, 1953). It is 
one of the most resistant accessions in field evaluations against $P$. fijiensis populations from around the world, with the exception of some isolates from the Pacific islands and Papua New Guinea (Fullerton and Olsen, 1995), which are considered as the center of origin of the disease (Carlier et al., 1996). In addition, var. Calcutta IV has shown resistance to crude extracts from P. fijiensis (Hernández, 1995; Okole and Schulz, 1997). The identification of PfAvr4 as a likely avirulence factor in var. Calcutta 4 provides a major advance for banana breeding programs aiming at increasing the level of resistance against black Sigatoka. Purified effector proteins can be used to identify other resistance genes and to facilitate rapid selection of resistant progeny from segregating populations. The current selection process in resistance breeding is inadequate as it exclusively relies on field evaluations, and is slow because black Sigatoka has a latent period of a month or longer and the banana cycling time, depending on the species, is approximately 10-15 months. Similar experiences slowed down resistance breeding in wheat to $Z$. tritici until the elucidation of its mating system showed single-gene inheritance of pathogenicity factors that facilitated more precise isolate characterizations and subsequent R-gene discovery (Goodwin, 2007; Goodwin et al., 2011; Kema et al., 2000, 1996; Ponomarenko et al., 2011). Other potential genes involved in pathogenicity are discussed in supporting Text S2.

QoIs represent a class of fungicides that initially showed impressive efficacy against many plant pathogens (Gisi et al., 2002). However, resistance evolved rapidly and soon rendered the compound of little use in multiple pathosystems (Brent and Hollomon, 1995). Diagnostic primers for the mitochondrial cytb gene showed that $P$. fijiensis is no exception, as the three commercial and frequently sprayed plantations were nearly or completely fixed for resistance. This is a remarkable shift compared to analyses performed during 2000-2003 when only part of the population was resistant (Amil et al., 2007). Interestingly, the San Carlos population, which was not sprayed with fungicides, is still entirely sensitive. This result 
suggests limited genetic exchange between these populations that are separated by about 100 $\mathrm{km}$. Nevertheless, even limited gene flow could have an impact on untreated areas. Because the selection pressure exerted by strobilurin is quite strong, the resistance frequency rapidly increases from a low number of resistant individuals to widespread resistance soon after fungicides are used, particularly since these compounds do not prevent sexual reproduction (Ware, 2006). This prompted us to process the $P$. fijiensis genome sequence with a bioinformatics pipeline to develop VNTR markers for rapid PCR-based population analyses to compare with a priori neutral markers. Nonetheless, some genetic differentiation occurs between the populations as already described earlier in Costa Rica using microsatellite markers (Halkett et al., 2010). However, because populations have not yet reached mutationdrift equilibrium, gene flow could not be estimated using classical genetic models based on genetic differentiation (Halkett et al., 2010). Fortunately, new indirect (Rieux et al., 2013b) and direct (Rieux et al., 2014) methods have been recently used to provide estimates of dispersal in $P$. fijiensis that could be integrated in theoretical and spatially explicit models to predict spatial patterns of fungicide resistance evolution under different management strategies.

The availability of the CIRAD86 genome sequence and the resequence data of CIRAD139 for $P$. fijiensis will blunt its continued threat to global production by facilitating the development of resistant cultivars in banana breeding programs. The rapid development of fungicide resistance and extreme variability of the $P$. fijiensis genome among isolates coupled with a high level of sexual reproduction make this pathogen highly adaptable to changing environmental conditions. Diversifying and increasing the level of host resistance in banana may be the only way to slow the devastation caused by this fungus in the future. 


\section{Materials and Methods}

\section{Fungal culture conditions and DNA extraction}

Pseudocercospora fijiensis isolate CIRAD86 (Mat1-1 mating type, originating from Cameroon in 1988) was chosen for sequencing because it is the epitype for the species, has been the subject of intensive analyses previously and is one parent of an existing mapping population (Manzo-Sánchez et al., 2008). CIRAD139a (Mat1-2, originating from Colombia in 1990) was used for resequencing. CIRAD86 is maintained at the CBS-KNAW Fungal Biodiversity Centre (CBS 120258).

Mycelia for DNA extraction were grown in 1-1 Erlenmeyer flasks containing $200 \mathrm{~mL}$ of PDB (potato dextrose broth; Becton Dickinson, NJ, USA) shaken at $120 \mathrm{rpm}$ at $28^{\circ} \mathrm{C}$. Mycelial mats produced during culture were filtered to remove the broth and lyophilized. Samples containing $50 \mathrm{mg}$ of lyophilized mycelia were placed in 2-mL tubes and ground with a Hybaid Ribolyser (model $\mathrm{n}^{\circ} \mathrm{FP} 120 \mathrm{HY}-230$ ) for $10 \mathrm{~s}$ at $2500 \mathrm{rpm}$ with a tungsten-carbide bead. DNA was extracted from the ground mycelia using the Wizard Magnetic DNA Purification system (Promega, Netherlands) for food according to instructions provided by the manufacturer.

\section{Genomic sequencing, genetic mapping, assembly and annotation}

Whole-genome shotgun sequencing and assembly of the $P$. fijiensis genome were done using Sanger sequencing of three different-sized libraries (3- and 8-kb plasmids, and 40$\mathrm{kb}$ fosmids) as described previously for Z. tritici (Goodwin et al., 2011) and other species (Ohm et al., 2010). The initial version 1 assembly was improved by aligning the physical scaffolds to a genetic linkage map constructed using Joinmap V 4.0 software (Van Ooijen and Voorrips, 2001) to analyze the segregation data for 322 markers that were scored on 135 
progeny of the cross between isolates CIRAD86 and CIRAD 139A (Manzo-Sánchez et al., 2008). For each molecular locus, a goodness-of-fit analysis was performed to test for deviation from the expected 1:1 segregation ratio at a 1\% significance level. Linkage groups were established using a minimum LOD score of 9.0 and final mapping was achieved by combining two or more linkage groups belonging to the same chromosome. The order of the markers on each chromosome was determined using a minimum LOD score of 1.0, recombination threshold of 0.4 , jump of 5.0, ripple value of 1 (default) and Haldane's mapping function as parameters. In cases of uncertainty, some markers were removed and the order was recalculated until a more stable order was achieved.

Three methods were used to identify the $P$. fijiensis repetitive sequences. Repeated sequences in the genome were identified de novo using RECON (Bao and Eddy, 2002) and the k-mer based method RepeatScout (Price et al., 2005). A custom set of repeats and the RepBase Update library of 234 fungal repeats (Jurka et al., 2005) were then used to mask the P. fijiensis genome using RepeatMasker (http://www.repeatmasker.org/) (Smit et al., 1996).

Repeat families with 10 or more elements identified by RepeatScout were annotated and classified into categories based on the presence of protein domains (BLAST (Altschul et al., 1990)). Structural features including Long Terminal Repeats (LTRs) and Terminal Inverted Repeats (TIRs) were verified using the EMBOSS (Rice et al., 2000) software package. Sequences with no known proteins or structural features were grouped into the unclassified category.

Identification and annotation of protein-coding genes were performed using the JGI Annotation Pipeline, which takes multiple inputs (scaffolds, ESTs, and known genes), runs several analytical tools for gene prediction and annotation, and deposits the results in the JGI 
fungal genome portal MycoCosm (http://jgi.doe.gov/fungi) (Grigoriev et al., 2013) for further analysis and manual curation.

Several gene-prediction programs falling into three general categories were used to annotate the repeat-masked assembly as described by Ohm et al. (Ohm et al., 2012). The resulting set of putative genes was then filtered for the best models based on EST and similarity support to produce a non-redundant representative set. This representative set of filtered gene models from the automated annotation pipeline was subject to further analysis and manual curation as described by Goodwin et al. for Z. tritici e and by Ohm et al. (Ohm et al., 2010) for more recently sequenced species. Measures of model quality included proportions of the models complete with start and stop codons ( $88 \%$ of models), those that were consistent with ESTs (30\% of models) and those supported by similarity with proteins from the NCBI NR database (74\% of models) as summarized in Table S8.

Functional annotations for all predicted gene models were made using SignalP (Nielsen et al., 1997), TMHMM e, InterProScan (Zdobnov and Apweiler, 2001), and BLASTp (Altschul et al., 1990) against the nr, SwissProt (http://www.expasy.org/sprot/), KEGG (Kanehisa et al., 2008) and KOG (Koonin et al., 2004) databases as described by Ohm et al. (Ohm et al., 2012). Multigene families were predicted with the Markov clustering algorithm (MCL) (Enright et al., 2002) to cluster the proteins using BLASTp alignment scores between proteins as a similarity metric. Functional annotations are summarized in Table S9. Manual curation of the automated annotations was performed using the web-based interactive editing tools of the JGI Genome Portal to assess predicted gene structures, assign gene functions, and report supporting evidence. Gene models predicted by the JGI annotation pipeline were also analyzed using the program Blast2GO (Conesa et al., 2005) with an Evalue of $<10^{-6}$. Blast2GO assigns GO terms based on the BLAST definitions. Comparisons 
between groups of genes for enrichment of GO terms were done by using Fisher's exact test implemented in the Blast2GO program.

Potential secreted proteins were identified with a python script made to run all gene models through SignalP 3.0 (Dyrlov Bendtsen et al., 2004) and subsequently filtered for proteins that had no transmembrane domains, no signal anchor motifs, were fewer than 300 amino acids in length and had at least 4 cysteine residues. The gene models that fulfilled these criteria were considered as potential Small Secreted Proteins (SSPs).

For re-sequencing of isolate CIRAD 139A, a paired-end library was made using the standard Illumina library prep protocol with NEB reagents. Average insert size of the library was 272 base pairs. Sequencing was done on an Illumina GAIIx in one lane of a 54-cycle paired-end run using 36-cycle version 5 SBS Kits. The flow cell was built using a version 4 paired-end cluster generation kit. Eventually, 37 million reads were obtained for a total yield of 4 gigabases. Paired reads were aligned to the P. fijiensis v2 Assembly reference scaffolds using GSNAP (2010-03-09 release), allowing up to 3 mismatches or 1 indel and with end trimming enabled. Uniquely aligned reads were then used to call variant sites using the Alpheus pipeline, requiring that a variant have support from at least 2 reads with an average quality of bases of at least phred 10 and at least $80 \%$ of the reads covering the site calling that variant. Nonsynonymous SNP differences were assessed against the coding regions in the $P$. fijiensis v2 FrozenGeneCatalog 20100402.

To survey the non-synonymous SNPs in the annotated protein set of CIRAD86, a simple analysis of functional bias in variant proteins was conducted using a ranking comparison approach. All genes were ranked based on their non-synonymous SNP count (normalized for coding sequence length) and two selected gene sets were compared with the whole-genome set. The two sets used for comparison were the 1500 most-variant proteins 
(Set V) and the 1500 least-variant proteins (Set C), taken from the list of genes ranked by non-synonymous SNP count. The annotation files for the P. fijiensis v2 Frozen Gene Catalog 20100402 were used as the source of GO terms for the genes. The ranked frequency of occurrence of GO terms in the gene annotations for the whole genome was compared with those for Sets C and V.

\section{Repetitive element analysis}

For each repetitive element family, a subset of elements with lengths within $50 \%$ of the longest element was aligned using clustalX (Larkin et al., 2007). These alignments were submitted to RIPCAL (Hane and Oliver, 2008) to determine the dinucleotide bias observed in repetitive elements. RIPCAL estimates 'RIP dominance' for each dinucleotide containing a cytosine. It is the ratio of a given dinucleotide (e.g., CA) to the sum of the other three dinucleotides (CG/CC/CT).

To test for isochores in the $P$. fijiensis genome, a contiguous stretch of sequence with an arbitrarily chosen average GC content of less than $45 \%$ was categorized as an AT block. Custom python scripts were used to calculate the percent GC across the genome, to generate AT blocks and to calculate the average percent GC across the AT blocks and those fewer than $500 \mathrm{bp}$ apart were merged into blocks of at least $1 \mathrm{~kb}$ in length, which were retained and analyzed for their composition and distribution of repetitive sequences and genes.

To estimate and compare the amount of RIP between the AT-rich blocks and the rest of the genome, a custom python script was written using a 500-bp sliding window with a step size of $100 \mathrm{bp}$. The amount of RIP was calculated as an index $(\mathrm{CpA}+\mathrm{TpG}) /(\mathrm{ApC}+\mathrm{GpT})$ and estimated separately for each of the AT-rich versus AT-poor regions in the genome. The RIP 
index measures the depletion of the RIP targets $\mathrm{CpA}$ and $\mathrm{TpG}$; thus, lower values of $(\mathrm{CpA}+\mathrm{TpG}) /(\mathrm{ApC}+\mathrm{GpT})$ are indicative of a higher degree of RIP [28].

To estimate the ages of transposon insertions, LTR retrotransposons were identified and annotated using the LTRharvest [100] and LTRdigest [101] modules in GenomeTools [102]. LTR sequences from these elements were aligned using ClustalW [99] and manually curated to estimate the numbers of mutations that had accumulated over time. All transition mutations were ignored in this analysis to remove the bias caused by RIP. Age of the LTR retrotransposons was calculated using the average rate of $1.09 \times 10^{-9}$ substitutions/site/year as proposed for fungal sequences [103].

To test whether the transposons in the $P$. fijiensis genome were unique, a comparison was made to TEs in the genome of $C$. fulvum, the only other sequenced fungus in the Capnodiales with an expanded genome. RepeatMasker (Price et al., 2005) was used to mask the $C$. fulvum genome using the repeat database from the $P$. fijiensis genome. The resulting file was parsed using the 80:80 rule of Wicker et al. (Wicker et al., 2007), i.e., $80 \%$ identity across $80 \%$ length to identify repeats in common between the two genomes. Another run was done at a 70:70 cutoff to allow for greater divergence generated by RIP.

\section{Generation and analysis of EST sequences}

The CIRAD86 strain was grown in three culture media for production of cDNA libraries: yeast-glucose broth as a rich medium (10 $\mathrm{g}$ of yeast extract and $30 \mathrm{~g}$ of glucose per liter); minimal nutrient medium ( $1 \mathrm{~g}$ of $\mathrm{KH}_{2} \mathrm{PO}_{4}, 1 \mathrm{~g}$ of $\mathrm{KNO}_{3}, 0.5 \mathrm{~g}$ of $\mathrm{MgSO}_{4} \cdot 7 \mathrm{H}_{2} \mathrm{O}, 0.5 \mathrm{~g}$ of $\mathrm{KCl}, 0.2 \mathrm{~g}$ of glucose, $0.2 \mathrm{~g}$ of sucrose per liter); and minimal nutrient medium without a nitrogen source (as above but without $\mathrm{KNO}_{3}$ ). Fungal mycelia were grown in each medium at $25^{\circ} \mathrm{C}$ for 10 days with a photoperiod of 12 hours using cool-white fluorescent light on a rotary 
shaker at $100 \mathrm{rpm}$. Mycelia derived from all three in vitro conditions were harvested by filtration and ground in liquid nitrogen. The RNA was isolated by the trizol method with the RNeasy kit (Qiagen, Netherlands) with 2 g of starting material. RNA quality and quantity were assessed by spectrophotometer and by gel electrophoresis according to standard procedures. For cDNA library construction, first-strand cDNA synthesis was done using polyA+ RNA, reverse transcriptase (SuperScriptII (Invitrogen, CA, USA)) and an oligo dTNotI primer (5' GACTAGTTCTAGATCGCGAGCGGCCGCCCT15VN 3'). Second-strand synthesis was done by E. coli DNA ligase, polymerase I and RNaseH before end repair with T4 DNA polymerase. The SalI adaptor (5' TCGACCCACGCGTCCG and 5' CGGACGCGTGGG) was ligated to the cDNA and digested with NotI before selecting the size range by gel electrophoresis. Sizes were $0.6-2 \mathrm{~kb}$ and 2-10 kb. The cDNA of $P$. fijiensis grown in yeast-glucose medium was divided into libraries CBBT and CBHU (0.6-2kb) and CBHT $(2-10 \mathrm{~kb})$. The cDNA from culture on minimal nutrient medium was divided into libraries CBBW and CBHX (0.6-2kb) and CBBU and CBHW (2-10kb), and for the libraries of culture on minimal nutrient medium without nitrogen source, cDNA was divided into libraries CBBX and $\mathrm{CBHY}(0.6-2 \mathrm{~kb})$. The size-selected inserts were cloned into the pCMVSPORT6 vector (Invitrogen) and digested with SalI and NotI. Ligated vectors were transformed into ElectroMAX T1 DH10B cells (Invitrogen).

Sequence reads from cDNA libraries were trimmed of vector, linker, adapter, poly$\mathrm{A} / \mathrm{T}$, and other artifact sequences with the Cross-match software. Internally developed software at the JGI-DOE identified short patterns and low-quality regions (Q15). The longest high-quality region of each read was counted as an EST. Clustering of ESTs was performed based on pairwise alignments generated using the Malign software, a modified version of the Smith-Waterman algorithm (Smith and Waterman, 1981), which was developed at the JGI for use in whole-genome shotgun assembly. ESTs sharing an alignment of at least $98 \%$ identity 
with 150-bp overlap were assigned to the same cluster. For each cluster of EST sequences, a consensus sequence was generated by running the Phrap software (Ewing et al., 1998; Ewing and Green, 1998). Comparison of the relative expression of genes in the different libraries was done by mapping ESTs to the whole set of predicted gene models and comparing their occurrences between a pair of libraries using Fisher's exact test. A P value of $<0.05$ was considered as statistically significant.

\section{Functional analysis of a putative effector protein}

Plantlets of $M$. acuminata ssp. burmannicoides var. Calcutta IV (recognized as a resistant standard for BLSD) were multiplied and rooted in vitro, whereas Cavendish "Grand Naine" tissue culture plants were hardened for three to four weeks in a greenhouse environment. Subsequently, all plantlets were grown for three months (small plants), and some plants of var. Calcutta IV and "Grand Naine" were grown for eight months (large plants) in a controlled-environment greenhouse compartment at $25^{\circ} \mathrm{C}$ with a relative humidity of $>80 \%$ and 16 hours of light per day.

Plants of tomato (Lycopersicum esculentum) cv. Moneymaker (MM), which has no known $e$ resistance genes $(C f 0)$, or an isogenic line previously transformed with the $C f 4$ resistance gene were grown under greenhouse conditions as described by Stergiopoulos et al. 2010 (Stergiopoulos et al., 2010) during 3-4 weeks.

The mature protein from the $P$. fijiensis putative effector gene PfAvr4 was produced heterologously by culturing Pichia pastoris isolate GS115 in a fermentor as described previously (Rooney et al., 2005, p. 2). Following production in the fermentor, the protein was further purified from excess liquid medium and smaller proteins by filtration through a 3-kDa membrane (Amicon Ultra-15 Centrifugal filter unit, Millipore, USA). 
Infiltration of the complete fermentor product or purified PfAVR4 protein into banana and tomato leaves was done by injection with a 1-ml syringe with no needle. Infiltrations on banana leaves were done at the original concentration and a six-fold higher concentration. For tomato, all infiltration materials were diluted fifty or seventy-five times prior to infiltration. Samples of the fermentor medium and water were infiltrated separately as negative controls for all plants. At each infiltration point, the observed water soaking of tissue was marked with a permanent marker. Observations were recorded with an Olympus C-8080 digital camera at 4 and 10 days post infiltration (dpi) on banana leaves, and at 6 dpi for tomato plants. Protein preparation and controls were infiltrated in at least two banana leaves from each genotype in small and large sizes, with at least 3 repetitions per leaf. Infiltrations in tomato plants were performed in at least 4 leaves with one repetition.

\section{Thermal denaturation assays}

The thermal denaturation method of Marmur and Doty (Marmur and Doty, 1962), performed basically as described by Smith et al. (Smith et al., 1995), was used to estimate G+C contents of DNA from P. fijiensis isolate CIRAD86 (CBS120258) plus that from the closely related banana pathogens $P$. musae (isolate UQ430; CBS121371) and P. eumusae (isolate CBS122457) as well as the previously sequenced $Z$. tritici isolate IPO323 (CBS115943) (Goodwin et al., 2011). Genomic DNA was isolated from cultures grown in PD broth at $25^{\circ} \mathrm{C}$ on a rotary shaker $(150 \mathrm{rpm})$ following the procedure described by Raeder and Broda (Raeder and Broda, 1985) and was dissolved in 0.1X SSC. Melting curves were obtained on a Perkin-Elmer $\lambda 25$ spectrophotometer equipped with a thermal programmer. The $\mathrm{G}+\mathrm{C}$ contents were calculated from the $\mathrm{T}_{\mathrm{m}}$ values (melting/transition temperature) derived from the peaks of the first derivatives of the melting curves (Owen et al., 1969). DNA from Candida parapsilosis isolate CBS604 ( $\mathrm{T}_{\mathrm{m}}$ in $0.1 \mathrm{x} \mathrm{SSC}, 70.6^{\circ} \mathrm{C}$ ) (Crous and Mourichon, 
2002) was used as a calibration control. Determinations were performed at least twice for each isolate.

\section{Phylogenetic analysis}

Phylogenetic analysis showing the placement of species within the Capnodiales with expanded genomes was done using Internal Transcribed Spacer regions (ITS). DNA sequences were downloaded from GenBank with the following accession numbers: AF181692 for Z. tritici, EU514233 for P. eumusae, EU514265 for P. musae and EU514248 for P. fijiensis or obtained from genome data available at the Fungal Genome portal at JGI. Sequence alignment was done using MUSCLE (Edgar, 2004) and the phylogenetic tree was generated with MEGA 6.0 (Tamura et al., 2013) using a Maximum Likelihood statistical method and the Tamura 3-parameter substitution model. Support for the nodes of the tree was estimated by bootstrapping with 1000 replications.

\section{Electrophoretic karyotyping}

Isolates of $P$. fijiensis grown for 3 weeks in $\mathrm{PD}$ broth at $28^{\circ} \mathrm{C}, 150 \mathrm{rpm}$, were blended and grown for $48 \mathrm{hrs}$ in the same medium at $20 \%$ strength amended with $1 \mu \mathrm{M}$ tricyclazole. Decanted culture was washed with $1 \mathrm{M}$ sorbitol, and added to $40 \mathrm{~mL}$ of $\mathrm{OM}$ buffer $(1.2 \mathrm{M}$ MgSO4, 10 mM K phosphate, pH 5.8 with 700 mg of glucanase (Sigma, Germany), 256 mg of yatalase (Takara, Japan), $7500 \mathrm{U}$ of $\beta$-glucuronidase (Sigma) and $0.8 \mathrm{~g}$ of driselase (Sigma)) in a ratio of $\sim 1: 3$ (mycelium:buffer). The enzymatic treatment was incubated at 33 ${ }^{\circ} \mathrm{C}$ and shaken at $50 \mathrm{rpm}$ for $4.5 \mathrm{hrs}$.

Protoplasts were filtered through a plastic mesh of $30 \mu \mathrm{m}$ and washed 3 times with 1 $\mathrm{M}$ sorbitol in sterile conditions. When the concentration was at least $1 \times 10^{8}$ per $\mathrm{mL}$, protoplasts were embedded in low-melting point (SeaKem® Gold) agarose at a final 
concentration of $0.5 \%$. Agarose plugs were treated with proteinase K as described previously (Mehrabi et al., 2007), washed with cold $50 \mathrm{mM}$ EDTA, and kept in the same solution at $4{ }^{\circ} \mathrm{C}$ until used.

Chromosomes of $P$. fijiensis were separated in a CHEF DR-II system (Bio-Rad, Netherlands). Small chromosomal bands were discriminated as described before (RodríguezGarcía et al., 2006) using the chromosomes of Saccharomyces cerevisiae (Bio-Rad) as a high molecular weight (HMW) standard. Large chromosomes were separated in a $0.8 \%$ lowmelting point (SeaKem ${ }^{\circledR}$ Gold) agarose gel, with $0.5 \%$ TBE buffer at $11^{\circ} \mathrm{C}$, and $50 \mathrm{~V}$ for 195 hrs with switching times from 4800 to $1800 \mathrm{sec}$, and $24 \mathrm{hrs}$ from 1800 to $1300 \mathrm{sec}$, followed by $20 \mathrm{hrs}$ at $60 \mathrm{~V}$ from 1300 to $800 \mathrm{sec}$, and finally $27 \mathrm{hrs}$ of 800 to $600 \mathrm{sec}$ at $80 \mathrm{~V}$. HMW standards were Schizosaccharomyces pombe and Hansenula wingei chromosomes (BioRad). Agarose gels were stained with SYBRGold (Invitrogen) and destained in water for 30 and 20 min, respectively, observed under a UV transilluminator and recorded with an Eagle Eye II (Stratagene) still video system.

\section{Whole-genome comparisons and synteny analyses}

Two tools, Circos (Krzywinski et al., 2009) and MUMmer (Kurtz et al., 2004), were used for structural analysis of the $P$. fijiensis genome. A nucleotide-based similarity search was done between the masked P. fijiensis and Z. tritici genomes and visualized using Circos (Krzywinski et al., 2009), whereas protein comparisons between the masked genomes were done using Promer (Kurtz et al., 2004). Proteins with greater than $60 \%$ identity were reported.

Proteins in P. fijiensis and Z. tritici with at least 50\% amino-acid identity and match length were grouped as orthologs using OrthoMCL (Li et al., 2003) and synteny blocks were determined using Orthocluster (Vergara and Chen, 2009). The Z. tritici protein dataset also 
was compared to two other phylogenetically distant Dothideomycetes in the order Pleosporales, P. nodorum and P. tritici-repentis.

\section{Fungicide sensitivity and population analyses}

To analyze the frequencies of molecular marker alleles and fungicide resistance within populations, four farms in Costa Rica were sampled during 2008 (Figure S8). San Pablo, Zent, and Cartagena are located in Limón province, where bananas are grown at high density on large plantations and diseases are controlled by using chemical fungicides. These farms are located in the main Costa Rica banana production area with approximate sizes of 285, 342 and 64 ha, respectively. A fourth farm, San Carlos (0.5 ha), is located in Alajuela province and is isolated geographically from the principal banana-production area. Leaf tissue was collected from ten banana plants from each farm. Ascospores were discharged from the pseudothecia onto water agar ("FRAC Methods for Monitoring Fungicide Resistance," 1991) and single ascospores were transferred immediately to $15 \times 100-\mathrm{mm}$ petri dishes filled with potato dextrose agar (PDA). Between eight to ten ascospores from each sample point were placed on each dish of PDA. After 4 days colonies were transferred to Mycophil agar (Becton Dickinson Microbiology Systems, Cockeysville, MD) and incubated for 15 days at $25^{\circ} \mathrm{C}$ under continuous fluorescent light for colony growth and conidial production. Eventually, 649 isolates were collected and analyzed for phenotypic and molecular variability.

To obtain DNA for population genetics analyses, mycelia of 190 isolates from each of the three commercial plantations and of 95 isolates from the San Carlos population were harvested and lyophilized for 24 hours. Genomic DNA was extracted using the Wizard Magnetic DNA Purification System for Food Kit (Promega, Madison, WI, USA) according to the manufacturer's instructions and $2 \mu \mathrm{L}$ per sample were quantified using a NanoDrop ND1000 Spectrophotometer (NanoDrop Technologies, Wilmington, DE, USA). Mating type 
(Mat) PCR assays (Conde-Ferráez et al., 2007) were performed in a 50- $\mu \mathrm{L}$ total volume containing $50 \mathrm{ng}$ of template genomic DNA, $2 \mathrm{mM} \mathrm{MgCl} 2,600 \mu \mathrm{M}$ dNTPs, $5 \mu \mathrm{M}$ of each primer, and $0.4 \mathrm{U}$ of Taq DNA polymerase (Roche, Mannhein, Germany). Temperature cycling was carried out with the following program: $94^{\circ} \mathrm{C}$ for $2 \mathrm{~min}, 40$ cycles of $94^{\circ} \mathrm{C}$ for 1 $\min , 70^{\circ} \mathrm{C}$ for $30 \mathrm{~s}$ and $72^{\circ} \mathrm{C}$ for $1 \mathrm{~min}$, and a final elongation period of $10 \mathrm{~min}$ at $72^{\circ} \mathrm{C}$.

Analysis of VNTR markers was done as reported previously (Garcia et al., 2010). For genotyping strobilurin resistance, primers were developed on the basis of the G143A mutation in the cytb gene (Sierotzki et al., 2000) to identify sensitive and resistant $P$. fijiensis field strains (Table S10) in $20-\mu \mathrm{L}$ aliquots containing $50 \mathrm{ng}$ of template genomic DNA, $2 \mathrm{mM}$ $\mathrm{MgCl}_{2}, 600 \mu \mathrm{M} \mathrm{dNTPs}, 5 \mu \mathrm{M}$ of each primer and $0.4 \mathrm{U}$ of Taq DNA polymerase (Roche, Mannhein, Germany). Temperature cycling was conducted with the following program: $94^{\circ} \mathrm{C}$ for $2 \mathrm{~min}, 40$ cycles of $94^{\circ} \mathrm{C}$ for $1 \mathrm{~min}, 70^{\circ} \mathrm{C}$ for $30 \mathrm{~s}$ and $72^{\circ} \mathrm{C}$ for $1 \mathrm{~min}$, and a final elongation period of $10 \mathrm{~min}$ at $72^{\circ} \mathrm{C}$. Amplicons were separated by electrophoresis using $1.0 \%$ (for mat and cytb assays) or 3.0\% (for VNTRs) agarose gels containing $0.3 \mu \mathrm{g} / \mathrm{mL}$ ethidium bromide, in $0.5 \times \mathrm{TBE}$ buffer at $120 \mathrm{~V}$ for approximately $1 \mathrm{~h}$ (for mat and cytb assays) or $5 \mathrm{~h}$ (for VNTRs) and were visualized and photographed using a UV transilluminator and Eagle Eye II (Stratagene) still video system.

To analyze the data, frequencies of the two mating types within each population and in the overall sample were tested for deviation from a 1:1 ratio with $\chi^{2}$ tests. A molecular multilocus haplotype was constructed for each isolate by combining the allelic data at all five VNTR loci. Gene diversity within each population $\left(H_{S}\right)$ in total and by locus was calculated using GenAlEx 6.4 (Peakall and Smouse, 2006). Total diversity over the entire sample $\left(H_{T}\right)$, mean gene diversity within populations $\left(H_{S}\right)$, genetic differentiation among populations $\left(G_{S T}\right)$ and the corrected, standardized measure of genetic differentiation $\left(G{ }^{\prime}{ }_{S T}\right)$ were calculated using GENODIVE Beta version 2.0 (Meirmans and Van Tienderen, 2004). In all cases, $H_{T}$ 
CHAPTER 2

and $H_{S}$ refer to the unbiased estimates as developed by Nei (Nei, 1987). Pairwise estimates of $G{ }^{\prime}{ }_{S T}$ and of Jost's differentiation $(D)$ (Jost, 2008) also were calculated with GENODIVE.

Multilocus haplotype diversity was calculated with multilocus (http://www.bio.ic.ac.uk/evolve/software/multilocus/\#what). 


\section{Acknowledgements}

This research paper is dedicated to the memory of Mr. Romano Orlich MSc - deceased December 24, 2015 - previous president of the National Banana Cooperation of Costa Rica (Corbana), who greatly motivated our interest in banana research. The MusaForever strategic alliance between Wageningen University and Research Center (WUR) and Embrapa, the company of technological innovation linked to the Ministry of Agriculture, Livestock and Food Supply (Mapa) in Brazil facilitated the exchange of CF, SG and MS. We acknowledge the International Transit Center of Bioversity International at the Catholic University of Leuven, Belgium for providing var. Calcutta IV and CIRAD930 germplasm and Rahan Meristem Ltd. (Rosh Hanikra, Israel) for continuous support by providing numerous Cavendish Grand Naine tissue culture plants. Dr. Michael F. Seidl, Wageningen University, Laboratory of Phytopathology, provided additional bioinformatics support. 


\section{References}

Abadie, C., Zapater, M.-F., Pignolet, L., Carlier, J., Mourichon, X., 2008. Artificial inoculation on plants and banana leaf pieces with Mycosphaerella spp., responsible for Sigatoka leaf spot diseases. Fruits 63, 319-323.

Altschul, S.F., Gish, W., Miller, W., Myers, E.W., Lipman, D.J., 1990. Basic local alignment search tool. J. Mol. Biol. 215, 403-410.

Amil, A.F., Heaney, S.P., Stanger, C., Shaw, M.W., 2007. Dynamics of QoI Sensitivity in Mycosphaerella fijiensis in Costa Rica During 2000 to 2003. Phytopathology 97, 1451-1457. https://doi.org/10.1094/PHYTO-97-11-1451

Arango, R.E., Togawa, R.C., Carpentier, S.C., Roux, N., Hekkert, B.L., Kema, G.H.J., Souza, M.T., 2011. Genome-wide BAC-end sequencing of Musa acuminata DH Pahang reveals further insights into the genome organization of banana. Tree Genet. Genomes 1-8.

Arzanlou, M., Groenewald, J., Fullerton, R., Abeln, E., Carlier, J., Zapater, M.F., Buddenhagen, I., Viljoen, A., Crous, P., 2008. Multiple gene genealogies and phenotypic characters differentiate several novel species of Mycosphaerella and related anamorphs on banana. Persoonia Mol. Phylogeny Evol. Fungi 20, 19.

Bao, Z., Eddy, S.R., 2002. Automated de novo identification of repeat sequence families in sequenced genomes. Genome Res. 12, 1269.

Beveraggi, A., Mourichon, X., Salle, G., 1993. Study of host-parasite interaction in susceptible and resistant bananas inoculated with Cercospora fijiensis, pathogen of black leaf streak disease. Breed. Banana Plantains Resist. Dis. Pests CIRAD Montp. Fr. 171-192.

Bowen, J.K., Mesarich, C.H., Bus, V.G.M., Beresford, R.M., Plummer, K.M., Templeton, M.D., 2011. Venturia inaequalis: the causal agent of apple scab. Mol. Plant Pathol. 12, 105-122. https://doi.org/10.1111/j.1364-3703.2010.00656.x

Brent, K.J., Hollomon, D.W., 1995. Fungicide Resistance in Crop Pathogens: How Can it be Managed?, FRAC Monograph No. 1 (second, revised edition). ed. FUNGICIDE RESISTANCE ACTION COMMITTEE, CROPLIFE INTERNATIONAL, Brussels.

Cambareri, E.B., Jensen, B.C., Schabtach, E., Selker, E.U., 1989. Repeat-induced G-C to A-T mutations in Neurospora. Science 244, 1571-1575.

Carlier, J., Lebrun, M.H., Zapater, M.F., Dubois, C., Mourichon, X., 1996. Genetic structure of the global population of banana black leaf streak fungus, Mycosphaerella fijiensis. Mol. Ecol. 5, 499-510. https://doi.org/10.1111/j.1365-294X.1996.tb00342.x

Carlier, J., Zapater, M.F., Lapeyre, F., Jones, D.R., Mourichon, X., 2000. Septoria leaf spot of banana: A newly discovered disease caused by Mycosphaerella eumusae (anamorph Septoria eumusae). Phytopathology 90, 884-890.

Churchill, A.C.L., 2011. Mycosphaerella fijiensis, the black leaf streak pathogen of banana: progress towards understanding pathogen biology and detection, disease development, and the challenges of control. Mol. Plant Pathol. 12, 307-328. https://doi.org/10.1111/j.13643703.2010.00672.x

Conde-Ferráez, L., Waalwijk, C., Canto-Canché, B.B., Kema, G.H.J., Crous, P.W., James, A.C., Abeln, E.C.A., 2007. Isolation and characterization of the mating type locus of Mycosphaerella fijiensis, the causal agent of black leaf streak disease of banana. Mol. Plant Pathol. 8, 111-120. https://doi.org/10.1111/j.1364-3703.2006.00376.x

Condon, B.J., Leng, Y., Wu, D., Bushley, K.E., Ohm, R.A., Otillar, R., Martin, J., Schackwitz, W., Grimwood, J., MohdZainudin, N., Xue, C., Wang, R., Manning, V.A., Dhillon, B., Tu, Z.J., Steffenson, B.J., Salamov, A., Sun, H., Lowry, S., LaButti, K., Han, J., Copeland, A., Lindquist, E., Barry, K., Schmutz, J., Baker, S.E., Ciuffetti, L.M., Grigoriev, I.V., Zhong, S., Turgeon, B.G., 2013. Comparative genome structure, secondary metabolite, and effector coding capacity across Cochliobolus Pathogens. PLoS Genet 9, e1003233. https://doi.org/10.1371/journal.pgen.1003233 
Conesa, A., Götz, S., García-Gómez, J.M., Terol, J., Talón, M., Robles, M., 2005. Blast2GO: a universal tool for annotation, visualization and analysis in functional genomics research. Bioinforma. Oxf. Engl. 21, 3674-3676. https://doi.org/10.1093/bioinformatics/bti610

Cooley, R.N., Caten, C.E., 1991. Variation in electrophoretic karyotype between strains of Septoria nodorum. Mol. Gen. Genet. MGG 228, 17-23. https://doi.org/10.1007/BF00282442

Cox, E.C., Vocke, C.D., Walter, S., Gregg, K.Y., Bain, E.S., 1990. Electrophoretic karyotype for Dictyostelium discoideum. Proc. Natl. Acad. Sci. 87, 8247-8251. https://doi.org/10.1073/pnas.87.21.8247

Crous, P.W., Mourichon, X., 2002. Mycosphaerella eumusae and its anamorph Pseudocercospora eumusae spp. nov.: causal agent of eumusae leaf spot disease of banana. Sydowia 54, 35-43.

de Bellaire, L. de L., Fouré, E., Abadie, C., Carlier, J., 2010. Black leaf streak disease is challenging the banana industry. Fruits 65, 327-342.

de Wit, P.J., Mehrabi, R., van den Burg, H.A., Stergiopoulos, I., 2009. Fungal effector proteins: past, present and future. Mol. Plant Pathol. 10, 735-747.

de Wit, P.J.G.M., van der Burgt, A., Ökmen, B., Stergiopoulos, I., Abd-Elsalam, K.A., Aerts, A.L., Bahkali, A.H., Beenen, H.G., Chettri, P., Cox, M.P., Datema, E., de Vries, R.P., Dhillon, B., Ganley, A.R., Griffiths, S.A., Guo, Y., Hamelin, R.C., Henrissat, B., Kabir, M.S., Jashni, M.K., Kema, G., Klaubauf, S., Lapidus, A., Levasseur, A., Lindquist, E., Mehrabi, R., Ohm, R.A., Owen, T.J., Salamov, A., Schwelm, A., Schijlen, E., Sun, H., van den Burg, H.A., van Ham, R.C.H.J., Zhang, S., Goodwin, S.B., Grigoriev, I.V., Collemare, J., Bradshaw, R.E., 2012. The Genomes of the fungal plant pathogens Cladosporium fulvum and Dothistroma septosporum reveal adaptation to different hosts and lifestyles but also signatures of common ancestry. PLoS Genet 8, e1003088. https://doi.org/10.1371/journal.pgen.1003088

Dhillon, B., Feau, N., Aerts, A.L., Beauseigle, S., Bernier, L., Copeland, A., Foster, A., Gill, N., Henrissat, B., Herath, P., LaButti, K.M., Levasseur, A., Lindquist, E.A., Majoor, E., Ohm, R.A., Pangilinan, J.L., Pribowo, A., Saddler, J.N., Sakalidis, M.L., de Vries, R.P., Grigoriev, I.V., Goodwin, S.B., Tanguay, P., Hamelin, R.C., 2015. Horizontal gene transfer and gene dosage drives adaptation to wood colonization in a tree pathogen. Proc. Natl. Acad. Sci. U. S. A. 112, 3451-3456. https://doi.org/10.1073/pnas.1424293112

Dhillon, B., Gill, N., Hamelin, R.C., Goodwin, S.B., 2014. The landscape of transposable elements in the finished genome of the fungal wheat pathogen Mycosphaerella graminicola. BMC Genomics 15, 1132. https://doi.org/10.1186/1471-2164-15-1132

D'Hont, A., Denoeud, F., Aury, J.-M., Baurens, F.-C., Carreel, F., Garsmeur, O., Noel, B., Bocs, S., Droc, G., Rouard, M., Da Silva, C., Jabbari, K., Cardi, C., Poulain, J., Souquet, M., Labadie, K., Jourda, C., Lengellé, J., Rodier-Goud, M., Alberti, A., Bernard, M., Correa, M., Ayyampalayam, S., Mckain, M.R., Leebens-Mack, J., Burgess, D., Freeling, M., Mbéguié-A-Mbéguié, D., Chabannes, M., Wicker, T., Panaud, O., Barbosa, J., Hribova, E., Heslop-Harrison, P., Habas, R., Rivallan, R., Francois, P., Poiron, C., Kilian, A., Burthia, D., Jenny, C., Bakry, F., Brown, S., Guignon, V., Kema, G., Dita, M., Waalwijk, C., Joseph, S., Dievart, A., Jaillon, O., Leclercq, J., Argout, X., Lyons, E., Almeida, A., Jeridi, M., Dolezel, J., Roux, N., Risterucci, A.-M., Weissenbach, J., Ruiz, M., Glaszmann, J.-C., Quétier, F., Yahiaoui, N., Wincker, P., 2012. The banana (Musa acuminata) genome and the evolution of monocotyledonous plants. Nature 488, 213-217. https://doi.org/10.1038/nature1 1241

Duplessis, S., Cuomo, C.A., Lin, Y.-C., Aerts, A., Tisserant, E., Veneault-Fourrey, C., Joly, D.L., Hacquard, S., Amselem, J., Cantarel, B.L., Chiu, R., Coutinho, P.M., Feau, N., Field, M., Frey, P., Gelhaye, E., Goldberg, J., Grabherr, M.G., Kodira, C.D., Kohler, A., Kües, U., Lindquist, E.A., Lucas, S.M., Mago, R., Mauceli, E., Morin, E., Murat, C., Pangilinan, J.L., Park, R., Pearson, M., Quesneville, H., Rouhier, N., Sakthikumar, S., Salamov, A.A., Schmutz, J., Selles, B., Shapiro, H., Tanguay, P., Tuskan, G.A., Henrissat, B., Peer, Y.V. de, Rouzé, P., Ellis, J.G., Dodds, P.N., Schein, J.E., Zhong, S., Hamelin, R.C., Grigoriev, I.V., Szabo, L.J., Martin, F., 2011. Obligate biotrophy features unraveled by the genomic analysis of rust fungi. Proc. Natl. Acad. Sci. 108, 9166-9171. https://doi.org/10.1073/pnas.1019315108 
Dyrlov Bendtsen, J., Nielsen, H., von Heijne, G., Brunak, S., 2004. Improved prediction of signal peptides: SignalP 3.0. J. Mol. Biol. 340, 783-795.

Edgar, R.C., 2004. MUSCLE: multiple sequence alignment with high accuracy and high throughput. Nucleic Acids Res. 32, 1792-1797. https://doi.org/10.1093/nar/gkh340

Ellwood, S.R., Liu, Z., Syme, R.A., Lai, Z., Hane, J.K., Keiper, F., Moffat, C.S., Oliver, R.P., Friesen, T.L., 2010. A first genome assembly of the barley fungal pathogen Pyrenophora teres f. teres. Genome Biol. 11, R109.

Enright, A.J., Van Dongen, S., Ouzounis, C.A., 2002. An efficient algorithm for large-scale detection of protein families. Nucleic Acids Res. 30, 1575.

Eriksson, O.E., Winka, K., n.d. Supraordinal taxa of Ascomycota. Myconet 1.

Ewing, B., Green, P., 1998. Base-calling of automated sequencer traces usingPhred. II. error probabilities. Genome Res. 8, 186.

Ewing, B., Hillier, L.D., Wendl, M.C., Green, P., 1998. Base-calling of automated sequencer traces usingPhred. I. Accuracy assessment. Genome Res. 8, 175.

FRAC Methods for Monitoring fungicide resistance, 1991. . EPPO Bull. 21, 292-293. https://doi.org/10.1111/j.1365-2338.1991.tb01241.x

Friesen, T.L., Stukenbrock, E.H., Liu, Z., Meinhardt, S., Ling, H., Faris, J.D., Rasmussen, J.B., Solomon, P.S., McDonald, B.A., Oliver, R.P., 2006. Emergence of a new disease as a result of interspecific virulence gene transfer. Nat. Genet. 38, 953-956. https://doi.org/10.1038/ng1839

Fullerton, R.A., Olsen, T.L., 1995. Pathogenic variability in Mycosphaerella fijiensis Morelet, cause of black Sigatoka in banana and plantain. N. Z. J. Crop Hortic. Sci. 23, 39-48. https://doi.org/10.1080/01140671.1995.9513866

Garcia, S. a. L., Van der Lee, T. a. J., Ferreira, C.F., Te Lintel Hekkert, B., Zapater, M.-F., Goodwin, S.B., Guzmán, M., Kema, G.H.J., Souza, M.T., 2010. Variable number of tandem repeat markers in the genome sequence of Mycosphaerella fijiensis, the causal agent of black leaf streak disease of banana (Musa spp.). Genet. Mol. Res. GMR 9, 2207-2212. https://doi.org/10.4238/vol9-4gmr934

Gisi, U., Sierotzki, H., Cook, A., McCaffery, A., 2002. Mechanisms influencing the evolution of resistance to Qo inhibitor fungicides. Pest Manag. Sci. 58, 859-67. https://doi.org/12233175

Goodwin, S.B., 2007. Back to basics and beyond: increasing the level of resistance to Septoria tritici blotch in wheat. Australas. Plant Pathol. 36, 532-538. https://doi.org/10.1071/AP07068

Goodwin, S.B., Ben M'Barek, S., Dhillon, B., Wittenberg, A.H.J., Crane, C.F., Hane, J.K., Foster, A.J., Van der Lee, T.A.J., Grimwood, J., Aerts, A., Antoniw, J., Bailey, A., Bluhm, B., Bowler, J., Bristow, J., van der Burgt, A., Canto-Canché, B., Churchill, A.C.L., Conde-Ferràez, L., Cools, H.J., Coutinho, P.M., Csukai, M., Dehal, P., De Wit, P., Donzelli, B., van de Geest, H.C., van Ham, R.C.H.J., Hammond-Kosack, K.E., Henrissat, B., Kilian, A., Kobayashi, A.K., Koopmann, E., Kourmpetis, Y., Kuzniar, A., Lindquist, E., Lombard, V., Maliepaard, C., Martins, N., Mehrabi, R., Nap, J.P.H., Ponomarenko, A., Rudd, J.J., Salamov, A., Schmutz, J., Schouten, H.J., Shapiro, H., Stergiopoulos, I., Torriani, S.F.F., Tu, H., de Vries, R.P., Waalwijk, C., Ware, S.B., Wiebenga, A., Zwiers, L.-H., Oliver, R.P., Grigoriev, I.V., Kema, G.H.J., 2011. Finished genome of the fungal wheat pathogen Mycosphaerella graminicola reveals dispensome structure, chromosome plasticity, and stealth pathogenesis. PLoS Genet 7, e1002070. https://doi.org/10.1371/journal.pgen.1002070

Goyon, C., Faugeron, G., 1989. Targeted transformation of Ascobolus immersus and de novo methylation of the resulting duplicated DNA sequences. Mol. Cell. Biol. 9, 2818-2827.

Grayburn, W.S., Selker, E.U., 1989. A natural case of RIP: degeneration of the DNA sequence in an ancestral tandem duplication. Mol. Cell. Biol. 9, 4416-4421.

Grigoriev, I.V., Nikitin, R., Haridas, S., Kuo, A., Ohm, R., Otillar, R., Riley, R., Salamov, A., Zhao, X., Korzeniewski, F., Smirnova, T., Nordberg, H., Dubchak, I., Shabalov, I., 2013. MycoCosm portal: gearing up for 1000 fungal genomes. Nucleic Acids Res. gkt1183. https://doi.org/10.1093/nar/gkt1183

Halkett, F., Coste, D., Rivas Platero, G.G., Zapater, M.F., Abadie, C., Carlier, J., 2010. Genetic discontinuities and disequilibria in recently established populations of the plant pathogenic 
fungus Mycosphaerella fijiensis. Mol. Ecol. 19, 3909-3923. https://doi.org/10.1111/j.1365294X.2010.04774.X

Hane, J.K., Lowe, R.G.., Solomon, P.S., Tan, K.C., Schoch, C.L., Spatafora, J.W., Crous, P.W., Kodira, C., Birren, B.W., Galagan, J.E., others, 2007. Dothideomycete plant interactions illuminated by genome sequencing and EST analysis of the wheat pathogen Stagonospora nodorum. Plant Cell Online 19, 3347.

Hane, J.K., Oliver, R.P., 2008. RIPCAL: a tool for alignment-based analysis of repeat-induced point mutations in fungal genomic sequences. BMC Bioinformatics 9, 478. https://doi.org/10.1186/1471-2105-9-478

Hernández, N.R., 1995. In vitro and greenhouse selection of Musa for resistance to black Sigatoka (Mycosphaerella fijiensis Morelet). Selección in vitro y en invernadero de Musa resistente a la sigatoka negra (Mycosphaerella fijiensis Morelet). Infomusa. 4, 15-16.

Horns, F., Petit, E., Yockteng, R., Hood, M.E., 2012. Patterns of repeat-induced point mutation in transposable elements of basidiomycete fungi. Genome Biol. Evol. 4, 240-247. https://doi.org/10.1093/gbe/evs005

Howlett, B.J., Idnurm, A., Pedras, M.S., 2001. Leptosphaeria maculans, the causal agent of blackleg disease of Brassicas. Fungal Genet. Biol. FG B 33, 1-14. https://doi.org/10.1006/fgbi.2001.1274

Iwaguchi, S., Homma, M., Tanaka, K., 1990. Variation in the electrophoretic karyotype analysed by the assignment of DNA probes in Candida albicans. J. Gen. Microbiol. 136, 2433-2442.

Jonge, R. de, Bolton, M.D., Kombrink, A., Berg, G.C.M. van den, Yadeta, K.A., Thomma, B.P.H.J., 2013. Extensive chromosomal reshuffling drives evolution of virulence in an asexual pathogen. Genome Res. 23, 1271-1282. https://doi.org/10.1101/gr.152660.112

Jost, L., 2008. GST and its relatives do not measure differentiation. Mol. Ecol. 17, 4015-4026. https://doi.org/10.1111/j.1365-294X.2008.03887.x

Jurka, J., Kapitonov, V.V., Pavlicek, A., Klonowski, P., Kohany, O., Walichiewicz, J., 2005. Repbase Update, a database of eukaryotic repetitive elements. Cytogenet. Genome Res. 110, 462-467.

Kanehisa, M., Araki, M., Goto, S., Hattori, M., Hirakawa, M., Itoh, M., Katayama, T., Kawashima, S., Okuda, S., Tokimatsu, T., others, 2008. KEGG for linking genomes to life and the environment. Nucleic Acids Res. 36, D480-D484.

Kema, G., 1996. Histology of the pathogenesis of Mycosphaerella graminicola in wheat. Phytopathology 86, 777. https://doi.org/10.1094/Phyto-86-777

Kema, G.H.J., Verstappen, E.C.P., Todorova, M., Waalwijk, C., 1996. Successful crosses and molecular tetrad and progeny analyses demonstrate heterothallism in Mycosphaerella graminicola. Curr. Genet. 30, 251-258. https://doi.org/10.1007/s002940050129

Kema, G.H.J., Verstappen, E.C.P., Waalwijk, C., 2000. Avirulence in the Wheat Septoria tritici Leaf Blotch Fungus Mycosphaerella graminicola Is Controlled by a Single Locus. Mol. Plant. Microbe Interact. 13, 1375-1379. https://doi.org/10.1094/MPMI.2000.13.12.1375

Koonin, E.V., Fedorova, N.D., Jackson, J.D., Jacobs, A.R., Krylov, D.M., Makarova, K.S., Mazumder, R., Mekhedov, S.L., Nikolskaya, A.N., Rao, B.S., others, 2004. A comprehensive evolutionary classification of proteins encoded in complete eukaryotic genomes. Genome Biol. 5, R7.

Krzywinski, M., Schein, J., Birol, İ., Connors, J., Gascoyne, R., Horsman, D., Jones, S.J., Marra, M.A., 2009. Circos: an information aesthetic for comparative genomics. Genome Res. 19, 1639-1645.

Kurtz, S., Phillippy, A., Delcher, A.L., Smoot, M., Shumway, M., Antonescu, C., Salzberg, S.L., 2004. Versatile and open software for comparing large genomes. Genome Biol. 5, R12.

Labbé, J., Murat, C., Morin, E., Tuskan, G.A., Le Tacon, F., Martin, F., 2012. Characterization of Transposable Elements in the Ectomycorrhizal Fungus Laccaria bicolor. PLoS ONE 7, e40197. https://doi.org/10.1371/journal.pone.0040197

Larkin, M.A., Blackshields, G., Brown, N.P., Chenna, R., McGettigan, P.A., McWilliam, H., Valentin, F., Wallace, I.M., Wilm, A., Lopez, R., Thompson, J.D., Gibson, T.J., Higgins, D.G., 2007. Clustal W and Clustal X version 2.0. Bioinformatics 23, 2947-2948. https://doi.org/10.1093/bioinformatics/btm404 
Li, L., Stoeckert, C.J., Roos, D.S., 2003. OrthoMCL: identification of ortholog groups for eukaryotic genomes. Genome Res. 13, 2178-2189.

Ma, L.-J., van der Does, H.C., Borkovich, K.A., Coleman, J.J., Daboussi, M.-J., Di Pietro, A., Dufresne, M., Freitag, M., Grabherr, M., Henrissat, B., Houterman, P.M., Kang, S., Shim, W.-B., Woloshuk, C., Xie, X., Xu, J.-R., Antoniw, J., Baker, S.E., Bluhm, B.H., Breakspear, A., Brown, D.W., Butchko, R.A.E., Chapman, S., Coulson, R., Coutinho, P.M., Danchin, E.G.J., Diener, A., Gale, L.R., Gardiner, D.M., Goff, S., HammondKosack, K.E., Hilburn, K., Hua-Van, A., Jonkers, W., Kazan, K., Kodira, C.D., Koehrsen, M., Kumar, L., Lee, Y.-H., Li, L., Manners, J.M., Miranda-Saavedra, D., Mukherjee, M., Park, G., Park, J., Park, S.-Y., Proctor, R.H., Regev, A., Ruiz-Roldan, M.C., Sain, D., Sakthikumar, S., Sykes, S., Schwartz, D.C., Turgeon, B.G., Wapinski, I., Yoder, O., Young, S., Zeng, Q., Zhou, S., Galagan, J., Cuomo, C.A., Kistler, H.C., Rep, M., 2010. Comparative genomics reveals mobile pathogenicity chromosomes in Fusarium. Nature 464, 367-373. https://doi.org/10.1038/nature08850

Manning, V.A., Pandelova, I., Dhillon, B., Wilhelm, L.J., Goodwin, S.B., Berlin, A.M., Figueroa, M., Freitag, M., Hane, J.K., Henrissat, B., Holman, W.H., Kodira, C.D., Martin, J., Oliver, R.P., Robbertse, B., Schackwitz, W., Schwartz, D.C., Spatafora, J.W., Turgeon, B.G., Yandava, C., Young, S., Zhou, S., Zeng, Q., Grigoriev, I.V., Ma, L.-J., Ciuffetti, L.M., 2013. Comparative genomics of a plant-pathogenic fungus, Pyrenophora triticirepentis, Reveals Transduplication and the impact of repeat elements on pathogenicity and population divergence. G3 GenesGenomesGenetics 3, 41-63. https://doi.org/10.1534/g3.112.004044

Manzo-Sánchez, G., Zapater, M.-F., Luna-Martínez, F., Conde-Ferráez, L., Carlier, J., JamesKay, A., Simpson, J., 2008. Construction of a genetic linkage map of the fungal pathogen of banana Mycosphaerella fijiensis, causal agent of black leaf streak disease. Curr. Genet. 53, 299-311. https://doi.org/10.1007/s00294-008-0186-x

Marin, D.H., Romero, R.A., Guzman, M., Sutton, T.B., 2003. Black Sigatoka: An Increasing Threat to Banana Cultivation. Plant Dis. 87, 208-222.

Marmur, J., Doty, P., 1962. Determination of the base composition of deoxyribonucleic acid from its thermal denaturation temperature*. J. Mol. Biol. 5, 109-118.

Mehrabi, R., Taga, M., Kema, G.H., 2007. Electrophoretic and cytological karyotyping of the foliar wheat pathogen Mycosphaerella graminicola reveals many chromosomes with a large size range. Mycologia 99, 868-876.

Meirmans, P.G., Van Tienderen, P.H., 2004. GENOTYPE and GENODIVE: two programs for the analysis of genetic diversity of asexual organisms. Mol. Ecol. Notes 4, 792-794.

Migheli, Q., Berio, T., Gullino, M.L., Garibaldi, A., 1995. Electrophoretic karyotype variation among pathotypes of Fusarium oxysporum f.sp. dianthi. Plant Pathol. 44, 308-315. https://doi.org/10.1111/j.1365-3059.1995.tb02782.x

Nei, M., 1987. Molecular evolutionary genetics. Columbia University Press.

Nfor, T.D., Ajong, F.D., Nuincho, L.I., 2011. Evaluation of varietal response to black sigatoka caused by Mycosphaerella fijiensis Morelet in banana nursery. Int. Res. J. Plant Sci. 2, 299304.

Nielsen, H., Engelbrecht, J., Brunak, S., Von Heijne, G., 1997. Identification of prokaryotic and eukaryotic signal peptides and prediction of their cleavage sites. Protein Eng. 10, 1.

Ohm, R.A., de Jong, J.F., Lugones, L.G., Aerts, A., Kothe, E., Stajich, J.E., de Vries, R.P., Record, E., Levasseur, A., Baker, S.E., Bartholomew, K.A., Coutinho, P.M., Erdmann, S., Fowler, T.J., Gathman, A.C., Lombard, V., Henrissat, B., Knabe, N., Kües, U., Lilly, W.W., Lindquist, E., Lucas, S., Magnuson, J.K., Piumi, F., Raudaskoski, M., Salamov, A., Schmutz, J., Schwarze, F.W.M.R., vanKuyk, P.A., Horton, J.S., Grigoriev, I.V., Wösten, H.A.B., 2010. Genome sequence of the model mushroom Schizophyllum commune. Nat. Biotechnol. 28, 957-963. https://doi.org/10.1038/nbt.1643

Ohm, R.A., Feau, N., Henrissat, B., Schoch, C.L., Horwitz, B.A., Barry, K.W., Condon, B.J., Copeland, A.C., Dhillon, B., Glaser, F., Hesse, C.N., Kosti, I., LaButti, K., Lindquist, E.A., Lucas, S., Salamov, A.A., Bradshaw, R.E., Ciuffetti, L., Hamelin, R.C., Kema, G.H.J., Lawrence, C., Scott, J.A., Spatafora, J.W., Turgeon, B.G., de Wit, P.J.G.M., 
Zhong, S., Goodwin, S.B., Grigoriev, I.V., 2012. Diverse lifestyles and strategies of plant pathogenesis encoded in the genomes of eighteen Dothideomycetes fungi. PLoS Pathog 8, e1003037. https://doi.org/10.1371/journal.ppat.1003037

Okole, B.N., Schulz, F.A., 1997. Selection of Mycosphaerella fijiensis-resistant cell lines from microcross sections of banana and plantain. Plant Cell Rep. 16, 339-343. https://doi.org/10.1007/BF01088293

Oliva, R., Win, J., Raffaele, S., Boutemy, L., Bozkurt, T.O., Chaparro-Garcia, A., Segretin, M.E., Stam, R., Schornack, S., Cano, L.M., others, 2010. Recent developments in effector biology of filamentous plant pathogens. Cell. Microbiol. 12, 705-715.

Orbach, M.J., Vollrath, D., Davis, R.W., Yanofsky, C., 1988. An electrophoretic karyotype of Neurospora crassa. Mol. Cell. Biol. 8, 1469-1473. https://doi.org/10.1128/MCB.8.4.1469

Ortiz, R., Vuylsteke, D., 1994. Inheritance of black sigatoka disease resistance in plantain-banana (Musa spp.) hybrids. Theor. Appl. Genet. 89, 146-152. https://doi.org/10.1007/BF00225134

Owen, R., Hill, L., Lapage, S., 1969. Determination of DNA base compositions from melting profiles in dilute buffers. Biopolymers 7, 503-516.

Peakall, R., Smouse, P.E., 2006. genalex 6: genetic analysis in Excel. Population genetic software for teaching and research. Mol. Ecol. Notes 6, 288-295. https://doi.org/10.1111/j.14718286.2005.01155.x

Ploetz, R.C., 2004. The most important disease of a most important fruit. APSnet Educ. Cent. Marzo $1,1999$.

Ponomarenko, A., Goodwin, S., Kema, G.H., 2011. Septoria tritici blotch (STB). Plant Health Instr. https://doi.org/10.1094/PHI-I-2011-0407-01

Price, A.L., Jones, N.C., Pevzner, P.A., 2005. De novo identification of repeat families in large genomes. Bioinformatics 21, i351.

Raeder, U., Broda, P., 1985. Rapid preparation of DNA from filamentous fungi. Lett. Appl. Microbiol. 1, 17-20.

Rice, P., Longden, I., Bleasby, A., others, 2000. EMBOSS: the European molecular biology open software suite. Trends Genet. 16, 276-277.

Rieux, A., De Lapeyre De Bellaire, L., Zapater, M.-F., Ravigne, V., Carlier, J., 2013a. Recent range expansion and agricultural landscape heterogeneity have only minimal effect on the spatial genetic structure of the plant pathogenic fungus Mycosphaerella fijiensis. Heredity 110, 29-38. https://doi.org/10.1038/hdy.2012.55

Rieux, A., Lenormand, T., Carlier, J., de Lapeyre de Bellaire, L., Ravigné, V., 2013b. Using neutral cline decay to estimate contemporary dispersal: a generic tool and its application to a major crop pathogen. Ecol. Lett. 16, 721-730. https://doi.org/10.1111/ele.12090

Rieux, A., Soubeyrand, S., Bonnot, F., Klein, E.K., Ngando, J.E., Mehl, A., Ravigne, V., Carlier, J., de Lapeyre de Bellaire, L., 2014. Long-distance wind-dispersal of spores in a fungal plant pathogen: estimation of anisotropic dispersal kernels from an extensive field experiment. PLoS ONE 9, e103225. https://doi.org/10.1371/journal.pone.0103225

Rodríguez-García, C.M., Raigosa-Flores, N., Conde-Ferráez, L., Peraza-Echeverría, L., CantoCanché, B., James-Kay, A., 2006. Variation in electrophoretic karyotype among Mexican isolates of Mycosphaerella fijiensis. Can. J. Plant Pathol. 28, 236-241.

Romero, R.A., Sutton, T.B., 1997. Sensitivity of Mycosphaerella fijiensis, causal agent of black Sigatoka of banana, to propiconazole. Phytopathology 87, 96-100.

Rooney, H.C., van't Klooster, J.W., van der Hoorn, R.A., Joosten, M.H., Jones, J.D., de Wit, P.J., 2005. Cladosporium Avr2 inhibits tomato Rcr3 protease required for Cf-2-dependent disease resistance. Science 308, 1783-1786.

Rouxel, T., Grandaubert, J., Hane, J.K., Hoede, C., van de Wouw, A.P., Couloux, A., Dominguez, V., Anthouard, V., Bally, P., Bourras, S., Cozijnsen, A.J., Ciuffetti, L.M., Degrave, A., Dilmaghani, A., Duret, L., Fudal, I., Goodwin, S.B., Gout, L., Glaser, N., Linglin, J., Kema, G.H.J., Lapalu, N., Lawrence, C.B., May, K., Meyer, M., Ollivier, B., Poulain, J., Schoch, C.L., Simon, A., Spatafora, J.W., Stachowiak, A., Turgeon, B.G., Tyler, B.M., Vincent, D., Weissenbach, J., Amselem, J., Quesneville, H., Oliver, R.P., Wincker, P., Balesdent, M.-H., Howlett, B.J., 2011. Effector diversification within 
compartments of the Leptosphaeria maculans genome affected by Repeat-Induced Point mutations. Nat. Commun. 2, 202. https://doi.org/10.1038/ncomms1189

SanMiguel, P., Gaut, B., Tikhonov, A., Nakajima, Y., Bennetzen, J., 1998. The paleontology of intergene retrotransposons of maize. Nat. Genet. 20, 43-45.

Santana, M.F., Silva, J.C., Batista, A.D., Ribeiro, L.E., Silva, G.F. da, Araújo, E.F. de, Queiroz, M.V. de, 2012. Abundance, distribution and potential impact of transposable elements in the genome of Mycosphaerella fijiensis. BMC Genomics 13, 720. https://doi.org/10.1186/14712164-13-720

Schoch, C.L., Shoemaker, R.A., Seifert, K.A., Hambleton, S., Spatafora, J.W., Crous, P.W., 2006. A multigene phylogeny of the Dothideomycetes using four nuclear loci. Mycologia 98, 1041-1052. https://doi.org/10.3852/mycologia.98.6.1041

Selker, E.U., Cambareri, E.B., Jensen, B.C., Haack, K.R., 1987. Rearrangement of duplicated DNA in specialized cells of Neurospora. Cell 51, 741-752.

Sierotzki, H., Parisi, S., Steinfeld, U., Tenzer, I., Poirey, S., Gisi, U., 2000. Mode of resistance to respiration inhibitors at the cytochrome bc1 enzyme complex of Mycosphaerella fijiensis field isolates. Pest Manag. Sci. 56, 833-841. https://doi.org/10.1002/15264998(200010)56:10<833::AID-PS200>3.0.CO;2-Q

Simmonds, N.W., 1953. Segregations in some diploid bananas. J. Genet. 51, 458-469. https://doi.org/10.1007/BF02982938

Smit, A.F., Hubley, R., Green, P., 1996. RepeatMasker Open-3.0.

Smith, K.M., Phatale, P.A., Bredeweg, E.L., Connolly, L.R., Pomraning, K.R., Freitag, M., 2006. Epigenetics of filamentous fungi, in: reviews in cell biology and molecular medicine. WileyVCH Verlag GmbH \& Co. KGaA, pp. 1063-1106.

Smith, M.T., De Cock, A., Poot, G., Steensma, H., 1995. Genome comparisons in the yeastlike fungal genus Galactomyces Redhead et Malloch. Int. J. Syst. Bacteriol. 45, 826-831.

Smith, T.F., Waterman, M.S., 1981. Identification of common molecular subsequences. J Mol Biol 147, 195-197.

Spanu, P.D., Abbott, J.C., Amselem, J., Burgis, T.A., Soanes, D.M., Stüber, K., Themaat, E.V.L. van, Brown, J.K.M., Butcher, S.A., Gurr, S.J., Lebrun, M.-H., Ridout, C.J., SchulzeLefert, P., Talbot, N.J., Ahmadinejad, N., Ametz, C., Barton, G.R., Benjdia, M., Bidzinski, P., Bindschedler, L.V., Both, M., Brewer, M.T., Cadle-Davidson, L., CadleDavidson, M.M., Collemare, J., Cramer, R., Frenkel, O., Godfrey, D., Harriman, J., Hoede, C., King, B.C., Klages, S., Kleemann, J., Knoll, D., Koti, P.S., Kreplak, J., LópezRuiz, F.J., Lu, X., Maekawa, T., Mahanil, S., Micali, C., Milgroom, M.G., Montana, G., Noir, S., O'Connell, R.J., Oberhaensli, S., Parlange, F., Pedersen, C., Quesneville, H., Reinhardt, R., Rott, M., Sacristán, S., Schmidt, S.M., Schön, M., Skamnioti, P., Sommer, H., Stephens, A., Takahara, H., Thordal-Christensen, H., Vigouroux, M., Weßling, R., Wicker, T., Panstruga, R., 2010. Genome expansion and gene loss in powdery mildew fungi reveal tradeoffs in extreme parasitism. Science 330, 1543-1546. https://doi.org/10.1126/science.1194573

Stergiopoulos, I., Cordovez, V., Ökmen, B., Beenen, H.G., Kema, G.H.J., de Wit, P.J.G.M., 2014. Positive selection and intragenic recombination contribute to high allelic diversity in effector genes of Mycosphaerella fijiensis, causal agent of the black leaf streak disease of banana. Mol. Plant Pathol. 15, 447-460. https://doi.org/10.1111/mpp.12104

Stergiopoulos, I., Van den Burg, H.A., Ökmen, B., Beenen, H.G., Van Liere, S., Kema, G.H.J., De Wit, P.J.G.M., 2010. Tomato $\mathrm{Cf}$ resistance proteins mediate recognition of cognate homologous effectors from fungi pathogenic on dicots and monocots. Proc. Natl. Acad. Sci. 107,7610 .

Stukenbrock, E.H., Jørgensen, F.G., Zala, M., Hansen, T.T., McDonald, B.A., Schierup, M.H., 2010. Whole-genome and chromosome evolution associated with host adaptation and speciation of the wheat pathogen Mycosphaerella graminicola. PLoS Genet 6, e1001189. https://doi.org/10.1371/journal.pgen.1001189

Talbot, N.J., Salch, Y.P., Ma, M., Hamer, J.E., 1993. Karyotypic variation within clonal lineages of the rice blast fungus, Magnaporthe grisea. Appl. Environ. Microbiol. 59, 585-593. 
Tamura, K., Stecher, G., Peterson, D., Filipski, A., Kumar, S., 2013. MEGA6: Molecular evolutionary genetics analysis version 6.0. Mol. Biol. Evol. 30, 2725-2729. https://doi.org/10.1093/molbev/mst197

Torres, J.M., Calderón, H., Rodríguez-Arango, E., Morales, J.G., Arango, R., 2012. Differential induction of pathogenesis-related proteins in banana in response to Mycosphaerella fijiensis infection. Eur. J. Plant Pathol. 133, 887-898. https://doi.org/10.1007/s10658-012-0012-7

van Esse, H.P., Bolton, M.D., Stergiopoulos, I., de Wit, P.J.G.M., Thomma, B.P.H.J., 2007. The chitin-binding Cladosporium fulvumeffector protein Avr4 is a virulence factor. Mol. Plant. Microbe Interact. 20, 1092-1101.

Van Ooijen, J.W., Voorrips, R.E., 2001. JoinMap® 3.0, Software for the calculation of genetic linkage maps. Plant Res. Int. Wagening. Neth. 1-51.

Vergara, I.A., Chen, N., 2009. Using OrthoCluster for the detection of synteny blocks among multiple genomes. Curr Protoc Bioinform 27, 1-6.

Ware, S.B., 2006. Aspects of sexual reproduction in Mycosphaerella species on wheat and barley: genetic studies on specificity, mapping, and fungicide resistance (Ph.D. Thesis). Wageningen Universiteit, The Netherlands.

Wicker, T., Keller, B., 2007. Genome-wide comparative analysis of copia retrotransposons in Triticeae, rice, and Arabidopsis reveals conserved ancient evolutionary lineages and distinct dynamics of individual copia families. Genome Res. 17, 1072-1081. https://doi.org/10.1101/gr.6214107

Wicker, T., Sabot, F., Hua-Van, A., Bennetzen, J.L., Capy, P., Chalhoub, B., Flavell, A., Leroy, P., Morgante, M., Panaud, O., Paux, E., SanMiguel, P., Schulman, A.H., 2007. A unified classification system for eukaryotic transposable elements. Nat. Rev. Genet. 8, 973-982. https://doi.org/10.1038/nrg2165

Wittenberg, A.H.J., van der Lee, T.A.J., Ben M'Barek, S., Ware, S.B., Goodwin, S.B., Kilian, A., Visser, R.G.F., Kema, G.H.J., Schouten, H.J., 2009. Meiosis Drives extraordinary genome plasticity in the haploid fungal plant pathogen Mycosphaerella graminicola. PLoS ONE 4, e5863. https://doi.org/10.1371/journal.pone.0005863

Zdobnov, E.M., Apweiler, R., 2001. InterProScan-an integration platform for the signaturerecognition methods in InterPro. Bioinformatics 17, 847.

Zolan, M.E., 1995. Chromosome-length polymorphism in fungi. Microbiol. Rev. 59, 686-698. 

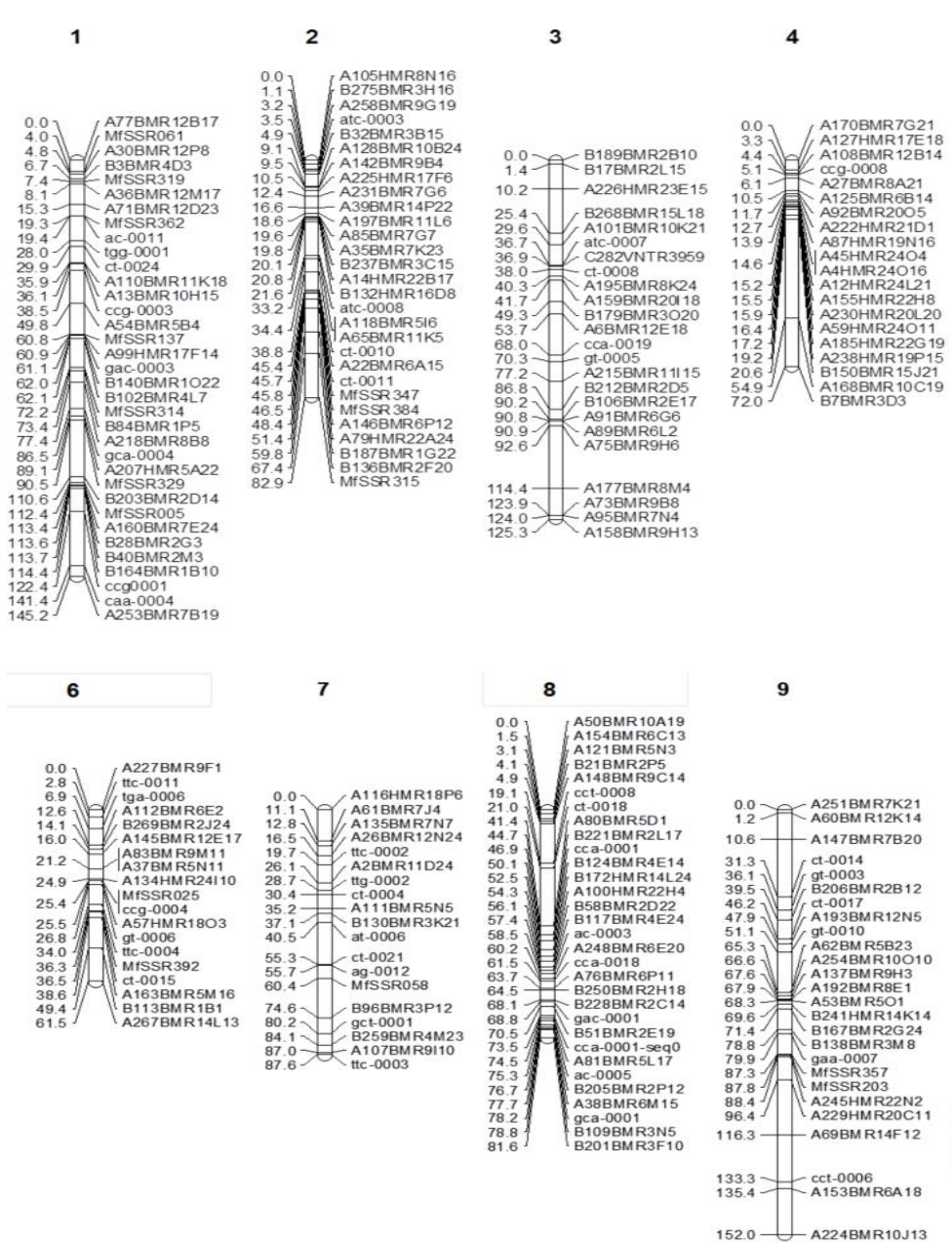

11

12

13

14

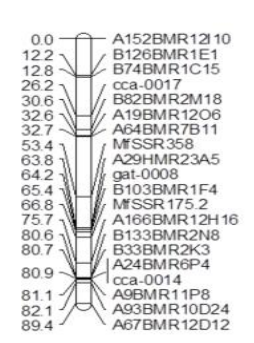

16
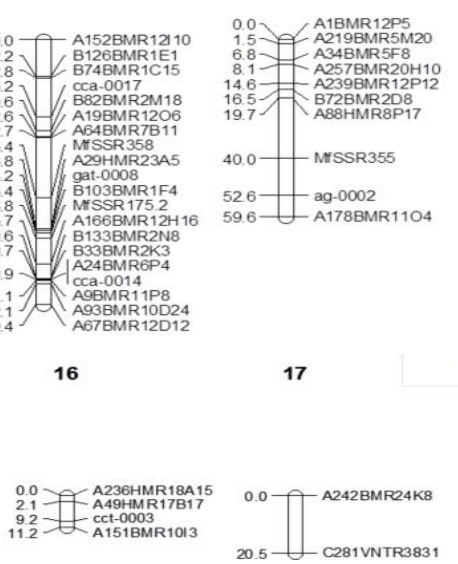

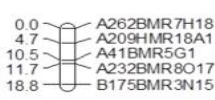

18
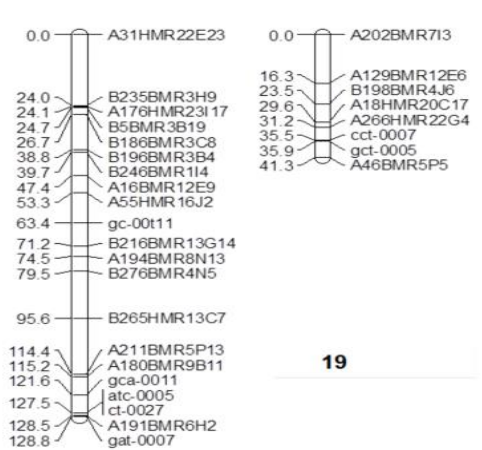

19

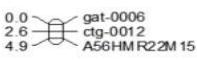
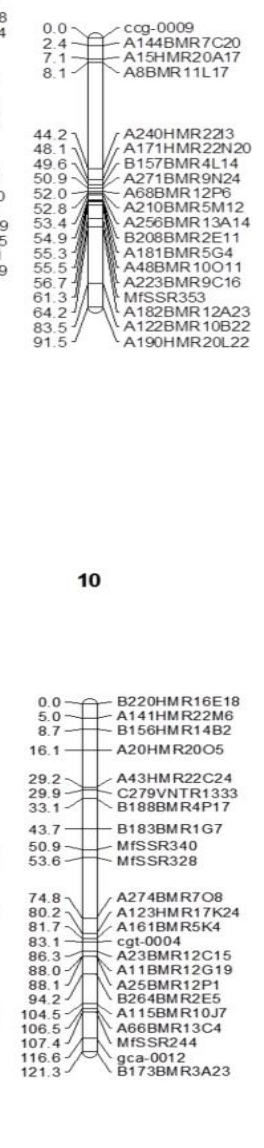

15

5

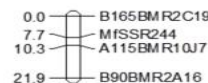

Figure 1. Genetic linkage map of Pseudocercospora fijiensis constructed from segregation data at 322 loci (233 DArT, 86 SSR and 3 minisatellite markers) among 135 individuals of a cross between the sequenced isolates CIRAD86 and CIRAD139A. The Diversity Arrays Technology (DArT) markers were named according to the output of proprietary DArT analysis software. For each of the 19 linkage groups (listed on top) the cumulative map distances (cM) as calculated using the Haldane mapping function are shown to the left. 


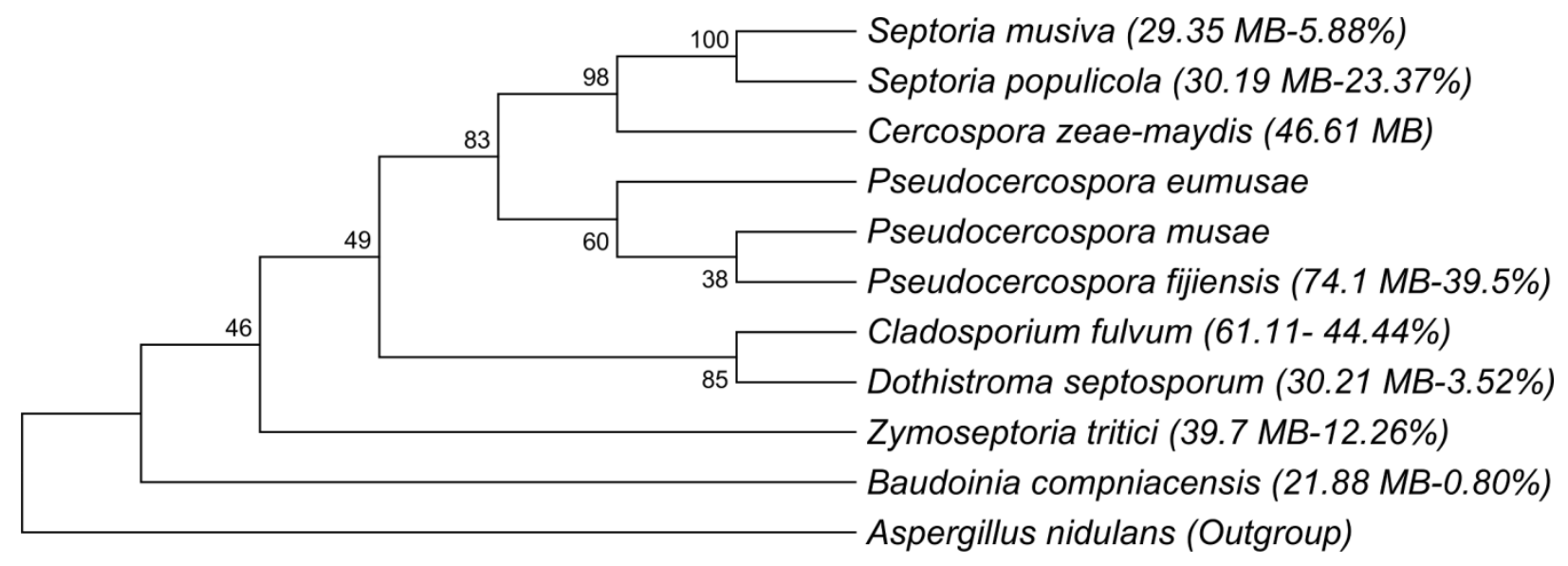

Figure 2. Phylogenetic analysis showing the placement of Dothideomycete species within the Capnodiales with expanded genomes. At least two genome expansions may have taken place; one leading to the banana pathogen Pseudocercospora fijiensis and one that contributed to its close relative the tomato pathogen Cladosporium fulvum. Genome sizes and percentages of the genome containing repeat elements are indicated in parentheses. 


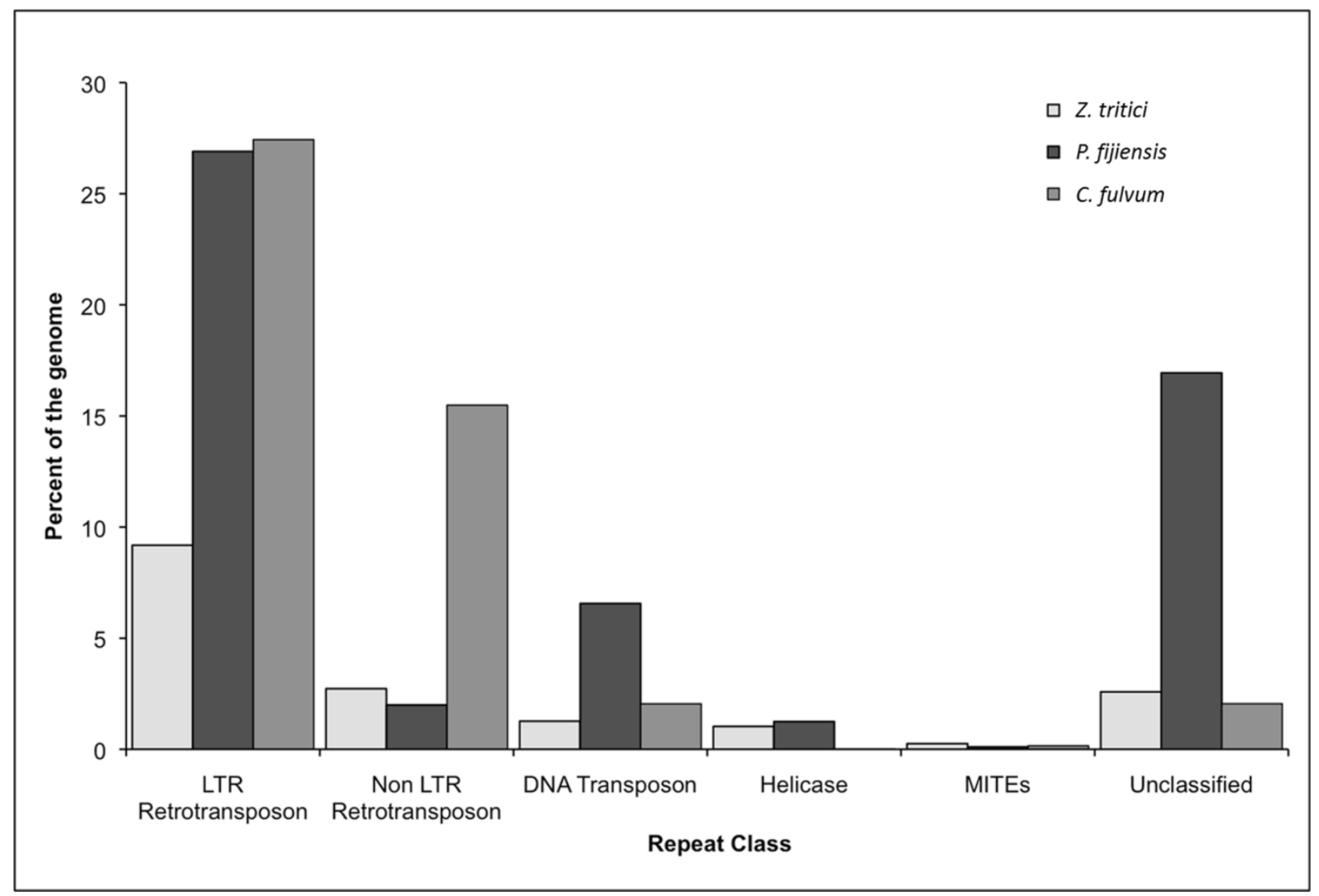

Figure 3. Comparison of repeat classes among Zymoseptoria tritici, the only Dothideomycete with a completely sequenced genome, Pseudocercospora fijiensis and Cladosporium fulvum, the only other Dothideomycete known to have a transposon-expanded genome. 


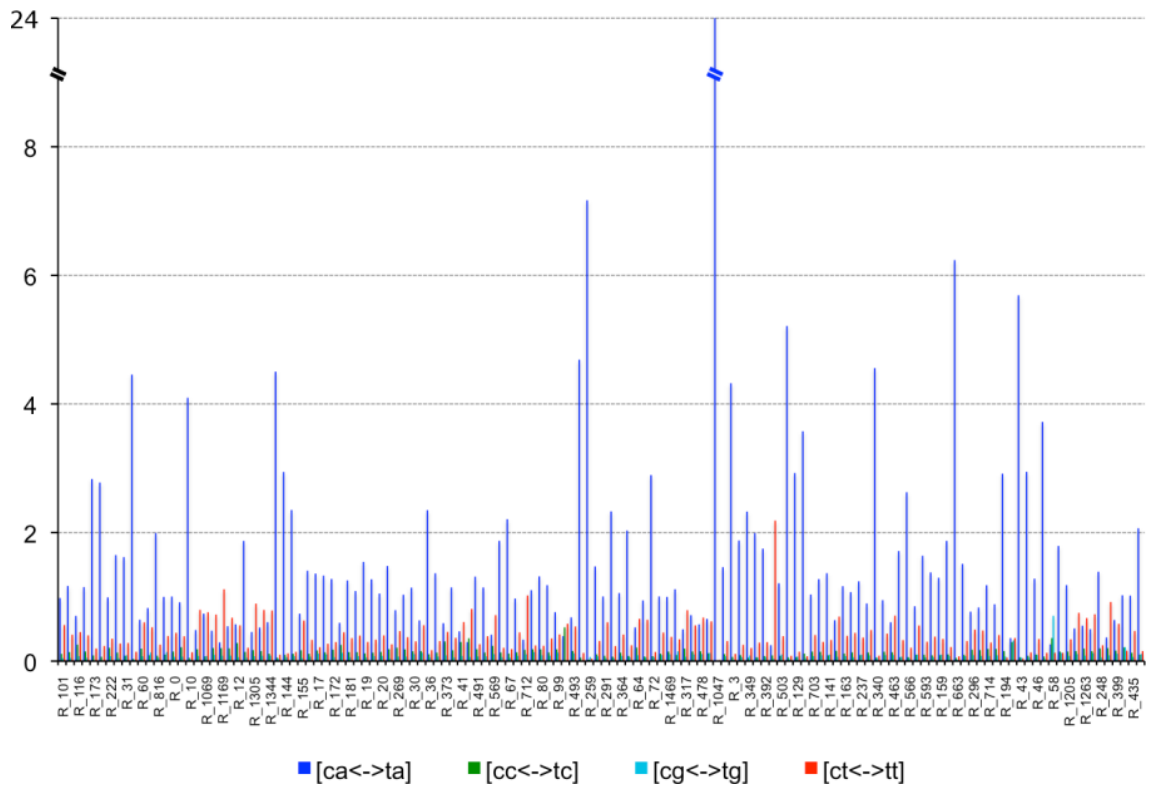

Figure 4. Repeat-induced point mutation (RIP) dinucleotide bias in Pseudocercospora fijiensis genome. A clear CA <-> TA dinucleotide bias is observed in P. fijiensis repetitive families, indicating that RIP likely occurs and mutates CA nucleotide pairs to CT. 
A
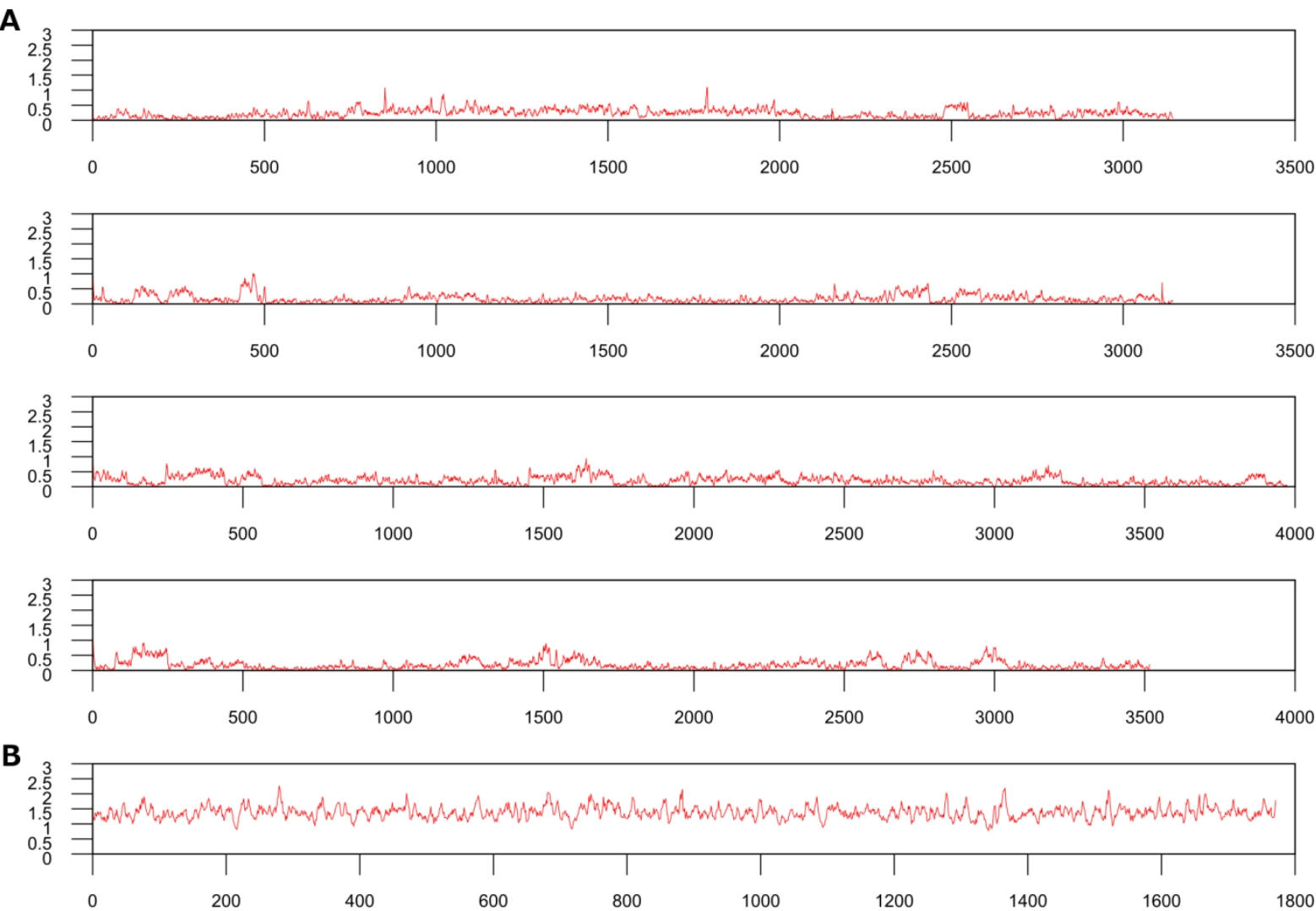

Figure 5. Comparison of the amount of repeat-induced point mutation (RIP) between AT-rich blocks and more GC-rich regions of the Pseudocercospora fijiensis genome as

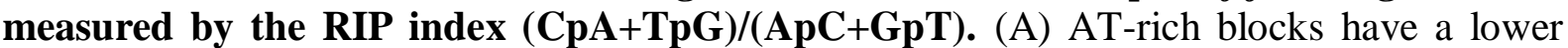
RIP index indicating a depletion of RIP-susceptible sites due to a higher frequency of RIP compared to (B) an AT-poor region (higher GC) of the genome, which has a higher RIP index reflecting very little RIP. Four AT-rich blocks are shown along with one AT-poor region for comparison. Length of each block in kilobases is shown along the $\mathrm{x}$-axis and the RIP index $(\mathrm{CpA}+\mathrm{TpG}) /(\mathrm{ApC}+\mathrm{GpT})$ is shown on the $\mathrm{y}$-axis. 

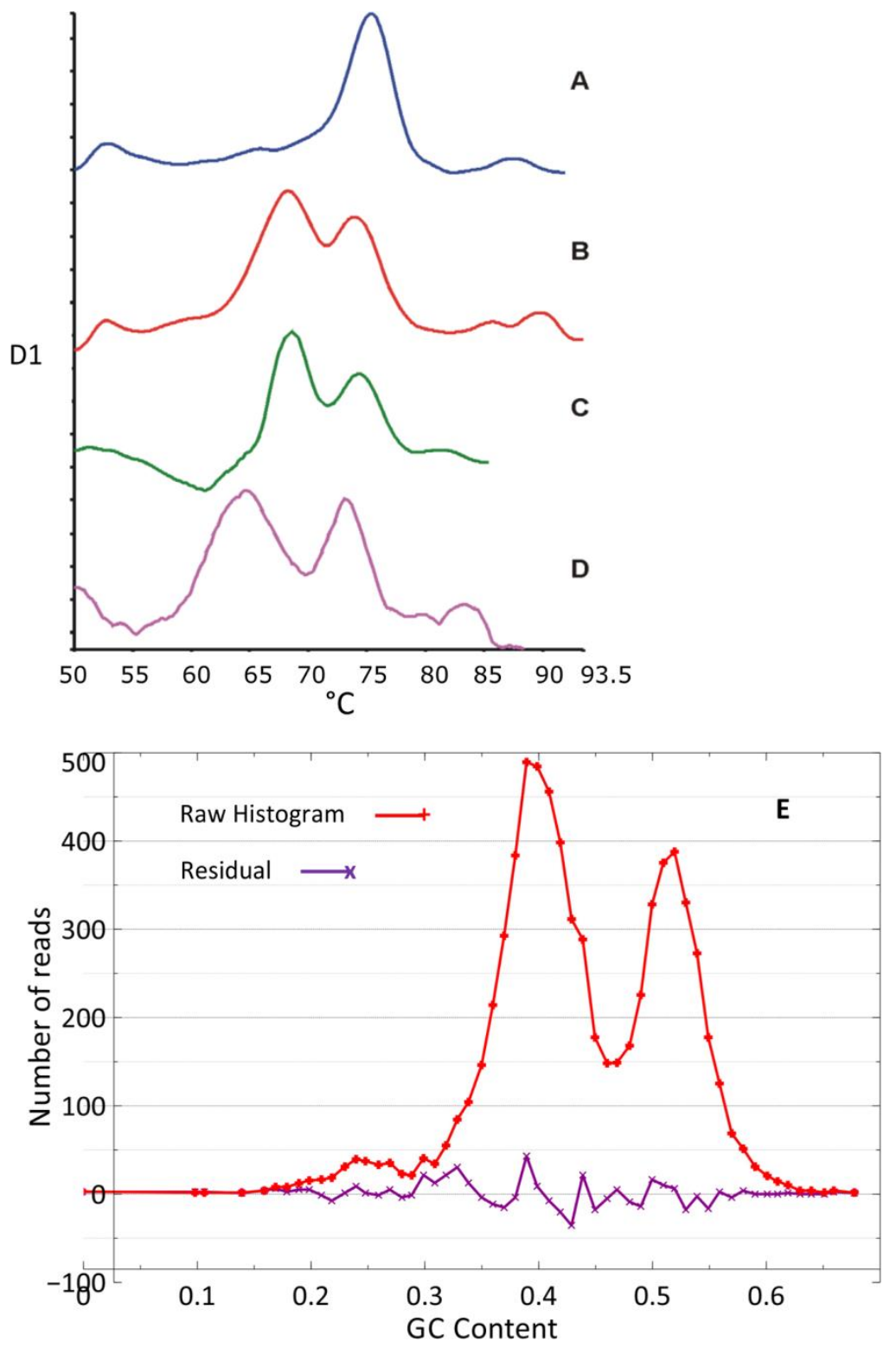

Figure 6. First-derivative graphs of melting curves of four different Dothideomycetes. Examples of first-derivative graphs of melting curves obtained for Zymoseptoria tritici (A), Pseudocercospora fijiensis (B), P. eumusae (C) and P. musae (D). E: A plot of G+C contents from sequence reads of $P$. fijiensis. This graph is very similar to the melting-curve analyses showing the difference in $\mathrm{G}+\mathrm{C}$ content between the genomes of $P$. fijiensis and the other banana pathogens versus the $Z$. tritici genome. 


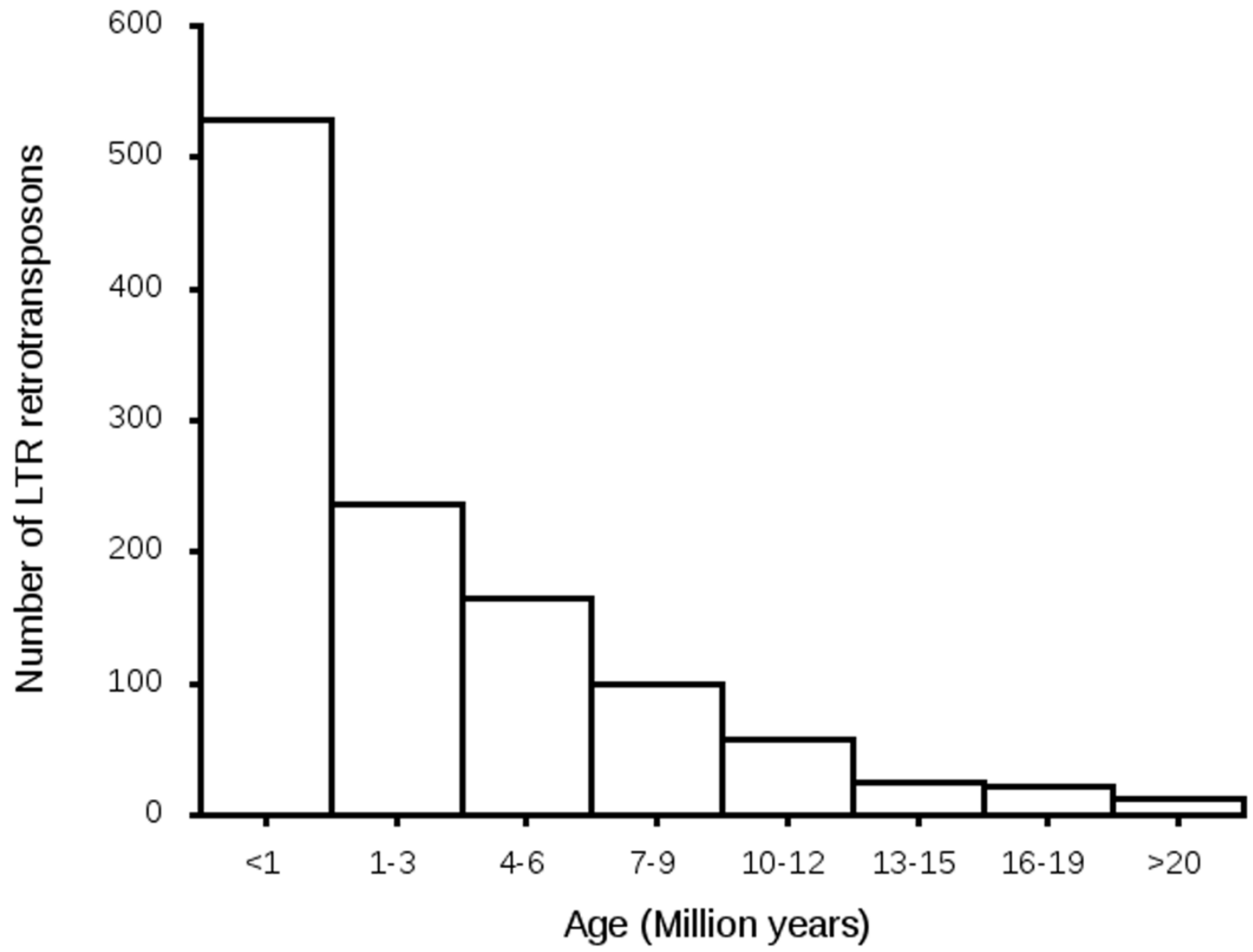

Figure 7. The numbers of long terminal repeat (LTR) retrotransposons in hypothetical age bins from less than one to more than 20 million years. Estimated age of each transposon was calculated using the number of differences between its left and right repeats. These are considered identical at the time of insertion so all changes are likely due to mutations that occurred after transposition. All transition mutations were excluded to minimize the effects of repeat-induced point mutation. 
A

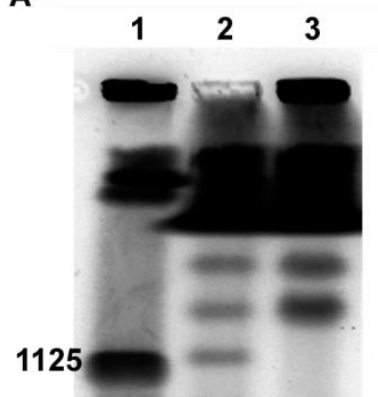

1020

945

825

750

610
565

450

365

285

225
B

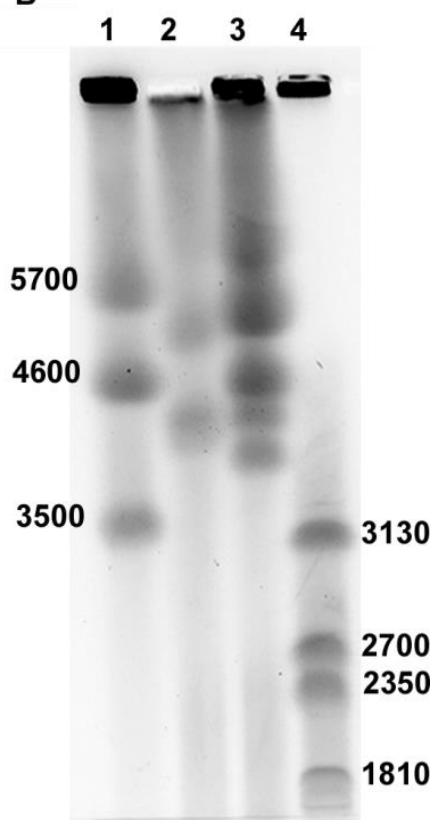

Figure 8. Electrophoretic karyotypes of two strains of Pseudocercospora fijiensis. A) Bands separated with conditions for small chromosomes. Lane 1, chromosomes from Saccharomyces cerevisiae as high-molecular-weight (HMW) marker; lane 2, strain CIRAD86; lane 3, strain E22. B) Bands separated under conditions to resolve medium and large chromosomes. Lane 1, chromosomes from Schizosaccharomyces pombe as HMW marker for large chromosomes; lane 2, strain CIRAD86; lane 3, strain E22; lane 4, chromosomes from Hansenula wingei as HMW marker for medium chromosomes in size. Marker sizes are in $\mathrm{Kb}$. 


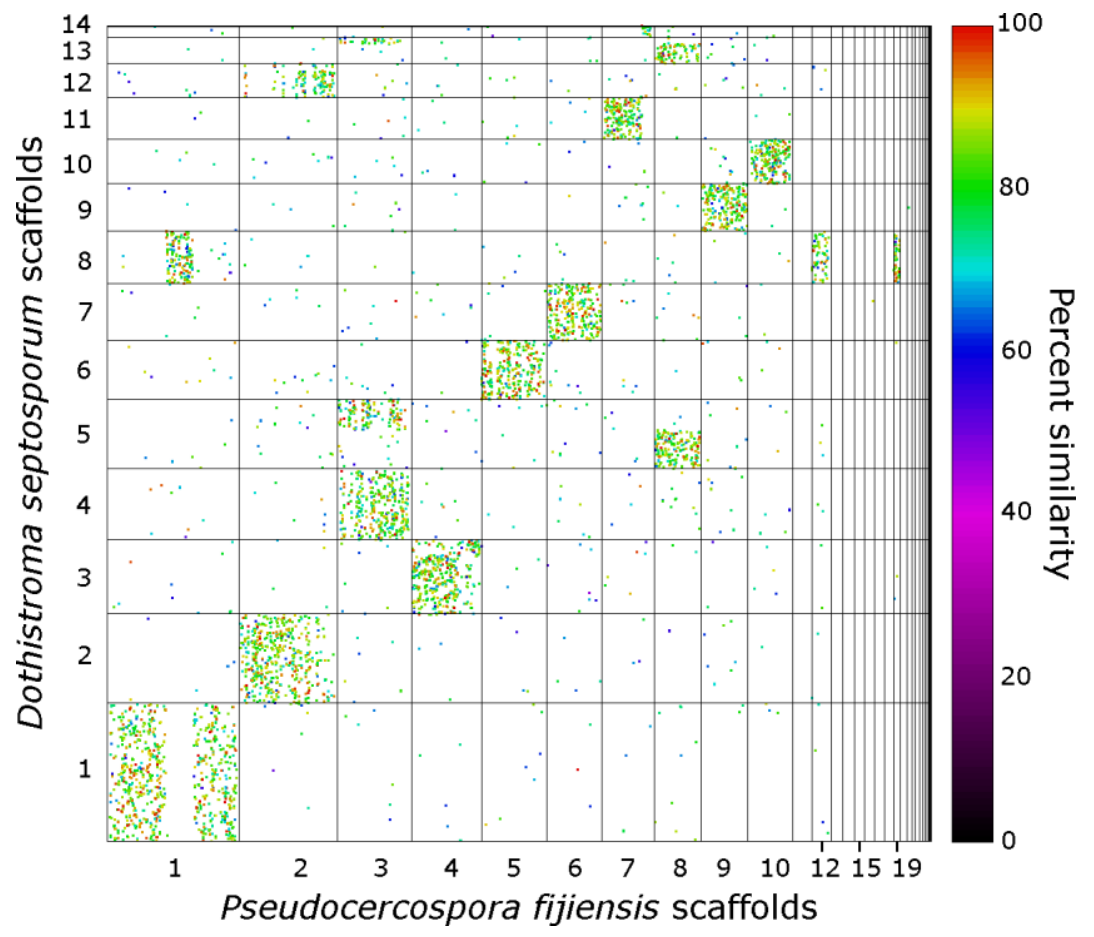

Figure 9. Dot plot showing mesosynteny between the scaffolds of Pseudocercospora fijiensis and Dothistroma septosporum. 


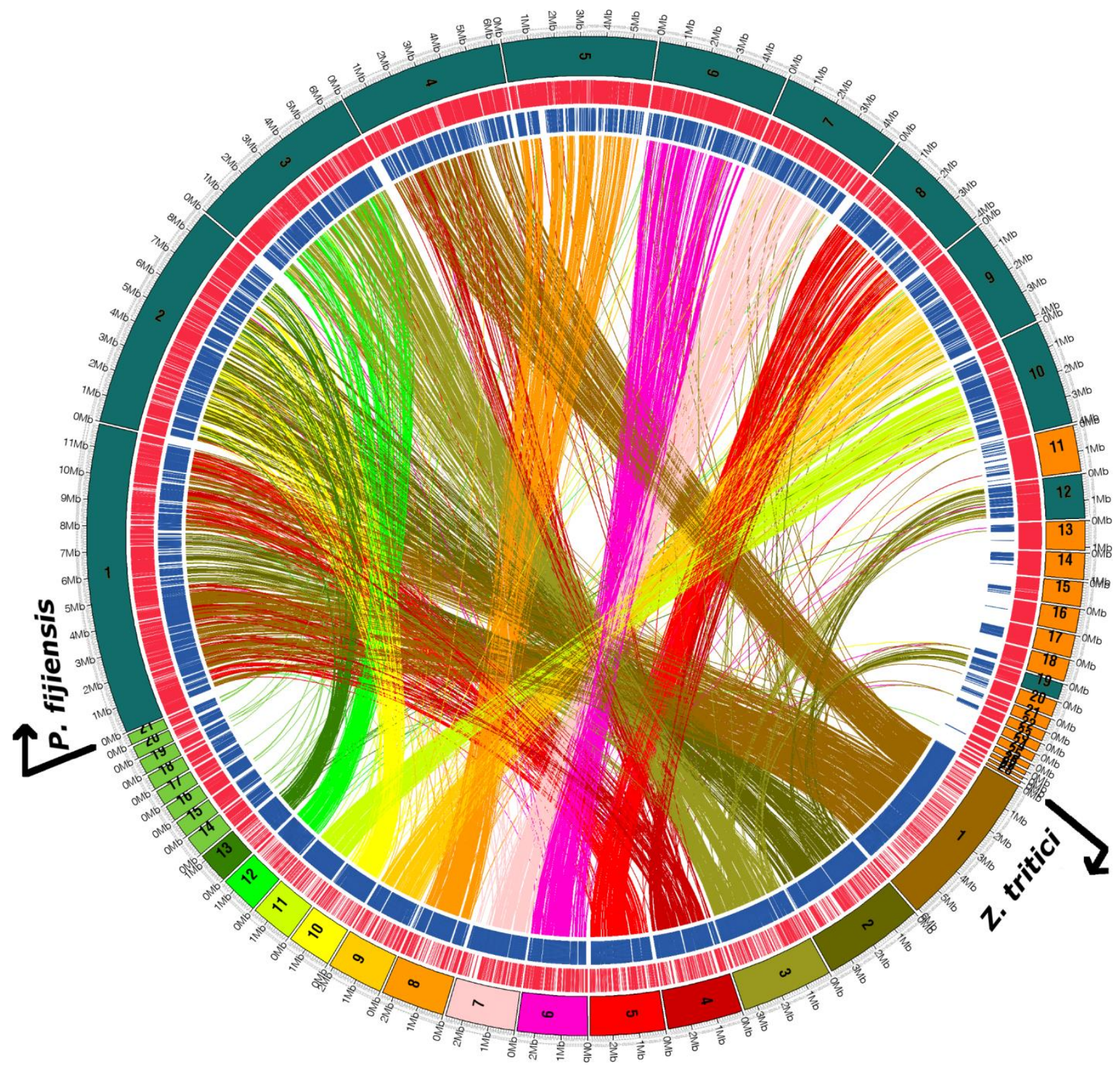

Figure 10. Genome-wide nucleotide comparison between Zymoseptoria tritici (lower half of the circle) and Pseudocercospora fijiensis (upper half of the circle). The longest 28 scaffolds from $P$. fijiensis are shown. Gene content is conserved but is scattered among different chromosomes between these two fungi. There were no significant hits to dispensable chromosomes of Z . tritici (14-21). The 12 major scaffolds of $P$. fijiensis showing synteny are labeled in dark blue-green and the other 16 scaffolds are labeled in orange. 
A
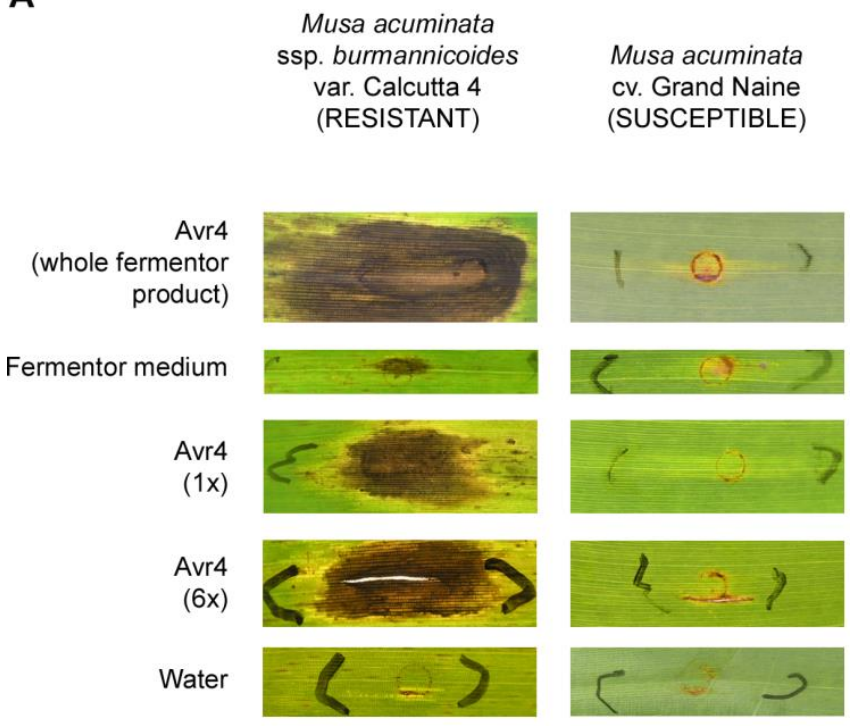

B

$\begin{array}{cc}\begin{array}{c}\text { Lycopersicum } \\ \text { esculentum } \\ \text { cv. Moneymaker } \\ \text { Cf-4 }\end{array} & \begin{array}{c}\text { Lycopersicum } \\ \text { esculentum } \\ \text { cv. Moneymaker }\end{array} \\ \text { (RESISTANT) } & \text { (SUS-0 } \\ \text { CfCEPTIBLE) }\end{array}$

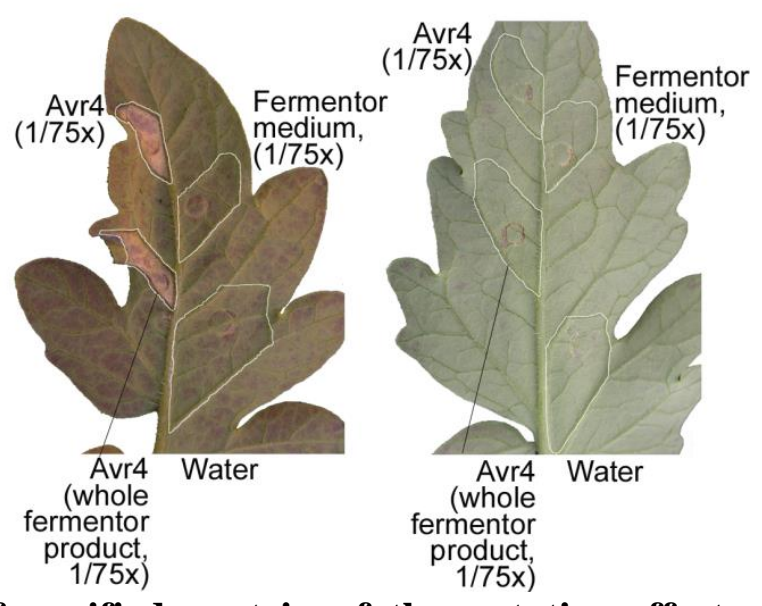

Figure 11. Infiltration of purified protein of the putative effector gene PfAvr4 from Pseudocercospora fijiensis into leaves of banana and tomato. A: Infiltrations into leaves of resistant and susceptible banana varieties. B: Infiltrations into leaves of tomato with or without the $C f 4$ resistance gene known to interact with PfAVR4. Experiments were done with crude fermentor product and concentrated or diluted product. Fermentor medium alone and water were used as controls. 


\section{Tables}

Table 1. Comparative genome statistics of the version 2 assembly of Pseudocercospora fijiensis, and several other sequenced fungi in the order Capnodiales.

\begin{tabular}{|c|c|c|c|c|c|c|c|}
\hline Genome statistic & $\begin{array}{c}\text { P. fijiensis } \\
\text { V } 2.0\end{array}$ & $\begin{array}{c}\text { C. fulvum } \\
\text { V } 1.0\end{array}$ & $\begin{array}{l}\text { Z. tritici } \\
\text { V } 2.0\end{array}$ & $\begin{array}{c}\text { B. } \\
\text { compniacensis } \\
\text { V1.0 }\end{array}$ & $\begin{array}{c}D . \\
\text { septosporum } \\
\text { V1.0 }\end{array}$ & $\begin{array}{c}\text { S. } \\
\text { populicola } \\
\text { V } 1.0\end{array}$ & $\begin{array}{c}\text { S. musiva } \\
\text { V } 1.0\end{array}$ \\
\hline Genome size & $74 \mathrm{MB}$ & $61.11 \mathrm{MB}$ & $40 \mathrm{MB}$ & $21.88 \mathrm{MB}$ & $30.21 \mathrm{MB}$ & $33.19 \mathrm{MB}$ & $29.35 \mathrm{MB}$ \\
\hline Scaffolds & 56 & 4865 & 21 & 19 & 20 & 502 & 72 \\
\hline Scaffolds $>50 \mathrm{~Kb}$ & 28 & N.A* & 21 & 17 & 14 & 141 & 13 \\
\hline Largest scaffold & $11.8 \mathrm{MB}$ & $0.53 \mathrm{MB}$ & $6.0 \mathrm{MB}$ & $2.03 \mathrm{MB}$ & 5.1 MB & $1.06 \mathrm{MB}$ & $5.11 \mathrm{MB}$ \\
\hline $\begin{array}{l}\text { Percent in scaffolds > } \\
50 \mathrm{~KB}\end{array}$ & 99.8 & N.A & 100 & N.A & N.A & N.A & N.A \\
\hline Gene models & 13,107 & 14,127 & 10,952 & 10,513 & 12,580 & 9,739 & 10,233 \\
\hline Coverage & $6.9 \times$ & N.A & $8.9 \times$ & $43 x$ & $34 x$ & $18 \mathrm{x}$ & $35 \mathrm{x}$ \\
\hline
\end{tabular}

*N.A. Data not available at respective genome site. 
Table 2. The repeat-induced point mutation (RIP) index calculated as $(\mathrm{CpA}+\mathrm{TpG}) /(\mathrm{ApC}+\mathrm{GpT})$ for genes $^{\mathrm{a}}$ and repeats ${ }^{\mathrm{a}}$ in AT-poor and-rich regions of the Pseudocercospora fijiensis genome.

\begin{tabular}{c|r|r|r|r}
\hline & \multicolumn{2}{|c|}{ Number of genes in } & \multicolumn{2}{c}{ Number of repeats in } \\
\hline RIP index & AT-poor blocks & AT-rich blocks & AT-poor blocks & \multicolumn{1}{c}{ AT-rich blocks } \\
\hline 0.0 & 0 & 0 & 1 & 3 \\
\hline 0.5 & 80 & 134 & 343 & 7,062 \\
\hline 1.0 & 291 & 37 & 62 & 607 \\
\hline 1.5 & 8,699 & 128 & 30 & 2 \\
\hline 2.0 & 1,937 & 22 & 1 & 0 \\
\hline 2.5 & 18 & 0 & 1 & 0 \\
\hline 3.0 & 1 & 0 & 0 & 0 \\
\hline Total & 11,026 & 321 & 438 & 7,674 \\
\hline
\end{tabular}

${ }^{a}$ The minimum sequence cutoff length for this analysis was $500 \mathrm{bp}$. A lower RIP index indicates a higher frequency of RIP mutations and vice versa.

doi:10.1371/journal.pgen.1005876.t002 
Table 3. Comparison of selected gene families with potential roles in pathogenicity among five Dothideomycete fungi and the saprotrophic Sordariomycete Neurospora crassa.

\begin{tabular}{|c|c|c|c|c|c|c|}
\hline & $\begin{array}{c}\text { Pseudocercospora } \\
\text { fijiensis }\end{array}$ & $\begin{array}{c}\text { Zymoseptoria } \\
\text { tritici }\end{array}$ & $\begin{array}{c}\text { Parastagonospora } \\
\text { nodorum }\end{array}$ & $\begin{array}{l}\text { Pyrenophora } \\
\text { tritici-repentis }\end{array}$ & $\begin{array}{c}\text { Cochliobolus } \\
\text { heterostrophus }\end{array}$ & $\begin{array}{c}\text { Neurospora } \\
\text { crassa }\end{array}$ \\
\hline Peptidases & 189 & 187 & 381 & 265 & 248 & 168 \\
\hline Cutinases & 7 & 6 & 0 & 8 & 13 & 0 \\
\hline $\begin{array}{l}\text { Beta Glucosidase } \\
\text { activity }\end{array}$ & 9 & 2 & 6 & 13 & 13 & 3 \\
\hline Chitinases & 5 & 3 & 36 & 17 & 21 & 8 \\
\hline Chitin binding & 5 & 2 & 35 & 13 & 16 & 3 \\
\hline Cellulose binding & 4 & 0 & 18 & 46 & 55 & 26 \\
\hline Xylanases & 7 & 5 & 31 & 21 & 31 & 5 \\
\hline NRPS & 13 & 11 & 18 & 13 & 11 & 5 \\
\hline $\begin{array}{l}\text { Polyketide } \\
\text { synthases }\end{array}$ & 11 & 12 & 21 & 28 & 21 & 4 \\
\hline Map kinases & 5 & 5 & 12 & 5 & 4 & 4 \\
\hline Peroxidases & 29 & 22 & 32 & 26 & 28 & 10 \\
\hline $\begin{array}{l}\text { Carbohydrate } \\
\text { Metabolic process }\end{array}$ & 127 & 77 & 231 & 204 & 209 & 129 \\
\hline $\begin{array}{l}\text { O-glycosyl } \\
\text { hydrolase activity }\end{array}$ & 77 & 51 & 129 & 97 & 106 & 77 \\
\hline
\end{tabular}

doi:10.1371/journal.pgen.1005876.t003 


\section{Supplementary data}

\section{Text S1. Additional information on genome sequencing, assembly and EST support.}

\section{Genome sequencing and assembly}

Pseudocercospora fijiensis isolate CIRAD86 was sequenced using Sanger sequencing on ABI 3730XL capillary machines. Three libraries with different sized inserts (3- and 8-kb plasmids, and $40-\mathrm{kb}$ fosmids) were used as templates for the plasmid subclone sequencing process and both ends were sequenced. After trimming sequences for vector sequences and eliminating those with low quality, a total of 867,068 reads was assembled into 382 main genome scaffolds using a modified version of Arachne (Jaffe et al., 2003). The resulting $P$. fijiensis v1.0 draft assembly totaled 73.6 Mb at an average read depth coverage of $7.11 \times$. Almost $84 \%$ of the genome was contained in 10 scaffolds that were each at least $4.0 \mathrm{Mb}$ in length (Table S1).

Inclusion of the genetic map data to facilitate assembly of the physical genome sequence involved sequencing 288 markers and aligning them to the version 1 draft assembly. Among the 288 markers sequenced, 262 were mapped to the version 1 genome assembly while 26 markers did not map. The improved version of the genome sequence has an estimated size of $74 \mathrm{Mb}$, assembled into 56 scaffolds covering more than $99 \%$ of the genome. The largest scaffold is $11.8 \mathrm{Mb}$ in length and 28 scaffolds $(99.8 . \%)$ are larger than $50 \mathrm{~Kb}$. Only $0.6 \%$ of sequence bases are estimated to be in gaps as compared to $8.9 \%$ in version 1.0 .

Annotation of the v1.0 assembly using a variety of similarity-based and ab initio gene predictors (Ohm et al., 2012) yielded 10,316 genes. Annotation of the v2.0 assembly was with the same annotation pipeline but with additional filtering by mapping of the v1.0 gene catalog along with its manual curations. After filtering for EST support completeness and similarity to 
other species, 13,107 genes were structurally and functionally annotated. The average gene length in the version 2 assembly is 1,833 nt with 3.62 exons per gene; $88 \%$, are complete with start and stop codon, $74 \%$ have similarity support, and 49\% have Pfam domains (Table S2). Most of the gene models (96\%) are located in 12 scaffolds, numbers 1-10, 12 and 19. Gene density in these 12 scaffolds varies from 151 to 229 per Mb and drops from 2.2 to 94 genes per $\mathrm{Mb}$ for the remaining scaffolds larger than $0.5 \mathrm{Mb}$ (Table S1).

\section{EST support}

Sequencing of nine cDNA libraries from three in vitro conditions generated 32,394 ESTs: 10,733 from growth in rich medium (library MFEST-3); 11,685 from growth in minimal medium with nitrogen (library MFEST-4); and 9,976 from growth in minimal medium without nitrogen (library MFEST-5). Average read length was 711.8 bp and percent GC was just over 53\%, corresponding to the high-GC peak seen in thermal-denaturation assays.

Clustering of ESTs from all libraries using Cap3 yielded 3,306 contigs and 3,164 singletons. Mapping of the ESTs to the version 2 genome assembly provided support for 5,663 of the predicted gene models; $99 \%$ of these are located in 13 scaffolds. Some scaffolds such as 11 and 14 had a very low density of genes and mapped ESTs (Table S1).

Differences in the relative abundance of EST sequences among the three libraries provide some clues about gene expression under each culture condition. Under rich conditions (library MFEST-3), genes that were more abundant included those with GO terms related to metabolic processes such as carbohydrate catabolic processes (GO:0046365, GO:0019320), lipid metabolic processes (GO:0006629 GO:0045834), dicarboxylic acid metabolic process (GO:0043648) and small-molecule biosynthetic process (GO:0044281), among others. Some interesting genes expressed exclusively under rich conditions in library 3 include a polyketide 
synthase, a D-3-phosphoglycerate dehydrogenase related to cytochrome p450 of Neurospora crassa, a Ctr copper transporter family protein (Aspergillus clavatus NRRL 1), and an ABC multidrug transporter mdr2 protein.

Genes with more abundance in minimal medium in the presence of nitrogen (library MFEST-4) had GO terms related to membrane fraction type proteins (GO:0005624), alcohol metabolic process (GO:0006066), binding (GO:0005488) and biosynthetic processes (GO:0009058, GO:0046364, GO:0019319). Interesting genes in MFEST-4 include a homolog of hydrophobin 1, which is the highest-expressed gene in this library, an alcohol oxidase and an extracellular cell wall glucanase. Several highly expressed genes in library MFEST-4 also were expressed in library MFEST-5 including the hydrophobin 1 and the alcohol oxidase. Enriched GO terms in library MFEST-5 included, among others, drug transmembrane transporter activity (GO:0015238 GO:0015893), ion transmembrane transporter activity (GO:0015075, GO:0006812, GO:0006811 and others) and several terms related to nucleotide binding activities (GO:0001882, GO:0032553).

\section{References}

Jaffe DB, Butler J, Gnerre S, Mauceli E, Lindblad-Toh K, Mesirov JP, et al. 2003. WholeGenome sequence assembly for mammalian genomes: Arachne 2. Genome Res. 13: 91-96. doi:10.1101/gr.828403

Ohm RA, Feau N, Henrissat B, Schoch CL, Horwitz BA, Barry KW, et al. 2012. Diverse lifestyles and strategies of plant pathogenesis encoded in the genomes of eighteen Dothideomycetes fungi. PLoS Pathog. 8: e1003037. doi:10.1371/journal.ppat.1003037 


\section{Text S2. Additional potential pathogenicity-related genes present in the $P$. fijiensis} genome.

Other potential genes involved in pathogenicity could be identified from EST support under different conditions. Several interesting genes were expressed in the libraries collected in minimal medium with and without nitrogen, which resemble the conditions that the fungus might face in a natural environment, including a hydrophobin 1 and an alcohol oxidase. An alcohol oxidase is a pathogenicity factor in Cladosporium fulvum (Segers et al., 2001), and the class I hydrophobin, MPG1, is involved in the formation and attachment of appressoria in the rice pathogen Magnaporthe oryzae (Talbot et al., 1993). Hydrophobins allow fungi to escape their aqueous environment and mediate attachment of hyphae to hydrophobic surfaces, which is important during the initial steps of fungal pathogenesis, where the fungus must attach to the hydrophobic surface of the host before penetration and infection (Hektor and Scholtmeijer , 2005). Because P. fijiensis does not penetrate directly it must grow on the surface of a leaf until it finds a stoma. The hydrophobin protein possibly could be involved in attachment to the host during its epiphytic phase until it can locate a suitable stoma for penetration.

Additional potential pathogenicity-related genes were identified through analysis of the pathways for melanin biosynthesis. Melanin is essential for successful host penetration by several plant-pathogenic fungi (Kubo et al., 1985; Rasmussen and Hanau, 1989; Wolkow et al., 1983). Although there are different types of fungal melanin, many pathogenic fungi, e.g., Magnaporthe oryzae, Verticillium dahliae, Blastomyces dermatitidis and Sporothrix schenckii, synthesize their pigments from acetate through the 1,8-dihydroxynaphthalene (DHN)-melanin pathway (Bell and Wheeler, 1986). Both Z. tritici and P. fijiensis may use this pathway for melanin biosynthesis because the necessary genes are present in their genomes. However, in Z. tritici melanin does not appear to be important for pathogenicity 
since melanin-deficient mutants were still pathogenic (Choi and Goodwin, 2011a), while mutants with increased melanin production showed reduced pathogenicity (Choi and Goodwin, 2011b). The importance of melanin for pathogenicity of $P$. fijiensis is not known.

The DHN-melanin pathway may be important for $P$. fijiensis even if melanin is not involved in its pathogenicity. In addition to melanin, this pathway is used for the synthesis of juglone and 2,4,8-tetrahydroxytetralone, both of which are produced by $P$. fijiensis in large amounts (Stierle et al., 1991; Host et al., 2000) and are phytotoxic to the banana plant, most probably by acting on chloroplasts (Busogoro et al., 2004). Knowing the sequences of all of the genes involved in the synthesis of these metabolites will open the way for functional analyses, allowing tests of whether they have a role in pathogenesis. The only knowledge in this respect is the virulence test of two pigment-deficient isolates (Donzelli and Churchill 200 [15]. Both isolates were capable of penetrating, but only one of them was virulent.

\section{References}

Bell AA, Wheeler MH. 1986. Biosynthesis and functions of fungal melanins. Annu Rev Phytopathol. 24: 411-451.

Busogoro JP, Etamé JJ, Lognay G, Messiaen J, Cutsem P van, Lepoivre P. 2004. Analysis of the mechanisms of action of Mycosphaerella fijiensis toxins during the development of black leaf streak disease. Science Publishers, Inc.; 2004. pp. 171-181.

Butler M, Day A. 1998. Fungal melanins: a review. Can J Microbiol.44: 1115-1136.

Choi Y-E, Goodwin SB. 2011a. MVE1, encoding the velvet gene product homolog in Mycosphaerella graminicola, is associated with aerial mycelium formation, melanin biosynthesis, hyphal swelling, and light signaling. Appl Environ Microbiol. 77: 942-953. doi:10.1128/AEM.01830-10

Choi Y-E, Goodwin SB. 2011b. Gene encoding a c-type cyclin in Mycosphaerella graminicola is involved in aerial mycelium formation, filamentous growth, hyphal swelling, melanin biosynthesis, stress response, and pathogenicity. Mol Plant Microbe Interact. 24: 469-477. doi:10.1094/MPMI-04-10-0090

Donzelli BGG, Churchill ACL. 200. A quantitative assay using mycelial fragments to assess virulence of Mycosphaerella fijiensis. Phytopathology. 97: 916-929. doi:10.1094/PHYTO-978-0916

Hektor HJ, Scholtmeijer K. Hydrophobins: 2005. Proteins with potential. Curr Opin Biotechnol. 16: 434-439.

Hoss R, Helbig J, Bochow H. 2000. Function of Host and Fungal Metabolites in Resistance Response of Banana and Plantain in the Black Sigatoka Disease Pathosystem (Musa spp. Mycosphaerella fijiensis). $J$ Phytopathol. 148: 387-394. doi:10.1046/j.14390434.2000.00530.x 
Kubo Y, Suzuki K, Furusawa I, Yamamoto M. 1985. Melanin biosynthesis as a prerequisite for penetration by appressoria of Colletotrichum lagenarium: site of inhibition by melanininhibiting fungicides and their action on appressoria. Pestic Biochem Physiol. 23: 47-55.

Rasmussen J, Hanau R. 1989. Exogenous scytalone restores appressorial melanization and pathogenicity in albino mutants of Colletotrichum graminicola. Can J Plant Pathol. 11: 349352.

Romero-Martinez R, Wheeler M, Guerrero-Plata A, Rico G, Torres-Guerrero H. 2000. Biosynthesis and functions of melanin in Sporothrix schenckii. Infect Immun. 8: 3696. Stierle AA, Upadhyay R, Hershenhorn J, Strobel GA, Molina G. 1991. The phytotoxins of Mycosphaerella fijiensis, the causative agent of Black Sigatoka disease of bananas and plantains. Cell Mol Life Sci CMLS. 47: 853-859.

Segers G, Bradshaw N, Archer D, Blissett K, Oliver RP. 2001. Alcohol oxidase is a novel pathogenicity factor for Cladosporium fulvum, but aldehyde dehydrogenase is dispensable. Mol Plant Microbe Interact. 14: 367-377. doi:10.1094/MPMI.2001.14.3.367

Talbot NJ, Ebbole DJ, Hamer JE. 1993. Identification and characterization of MPG1, a gene involved in pathogenicity from the rice blast fungus Magnaporthe grisea. Plant Cell Online. 5: 1575 .

Wolkow P, Sisler H, Vigil E. 1983. Effect of inhibitors of melanin biosynthesis on structure and function of appressoria of Colletotrichum lindemuthianum. Physiol Plant Pathol. 23: 55-71. 


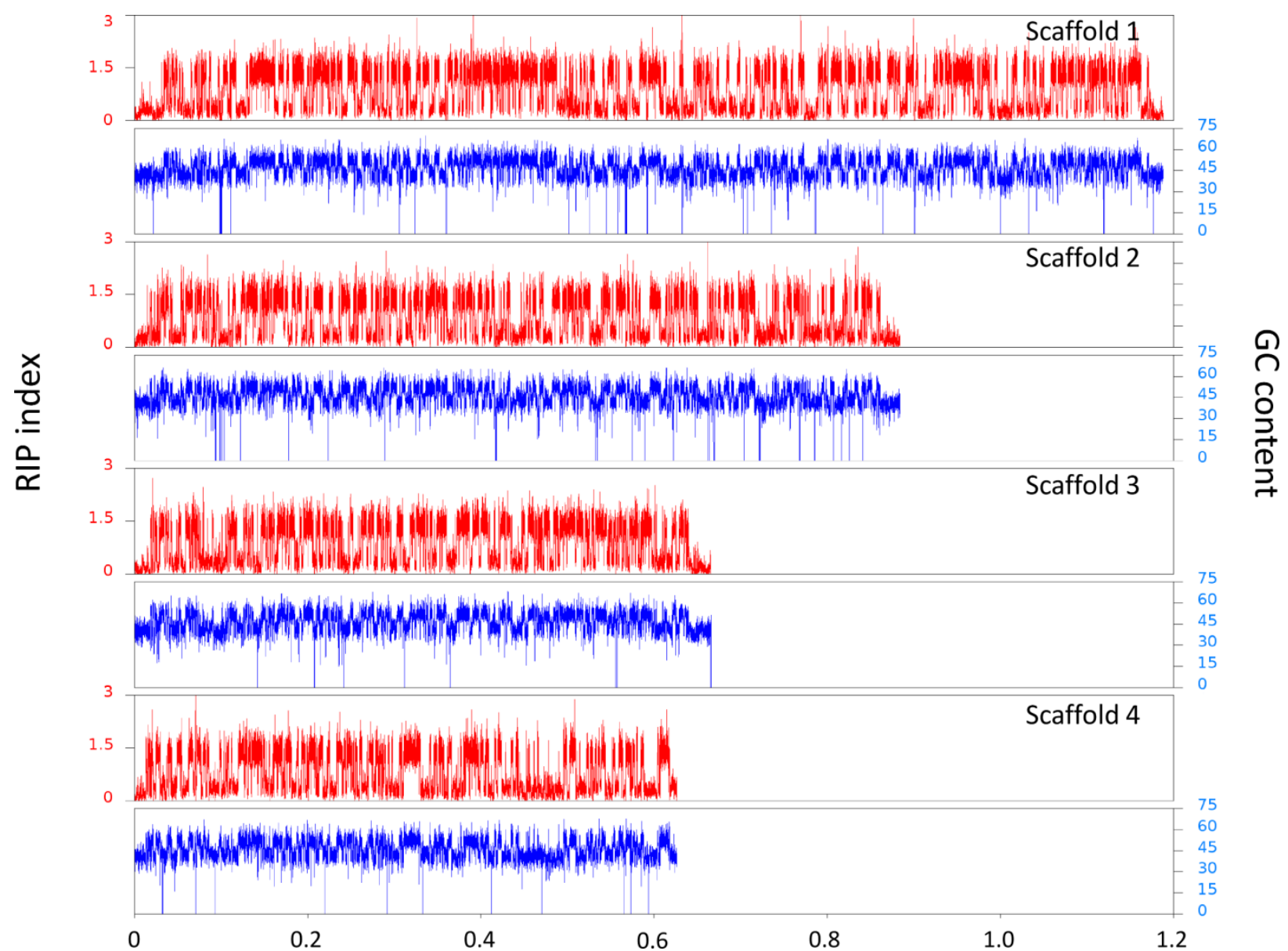

Figure S1. The strong positive association between RIP index (where a high index value indicates low RIP) and GC content shows that RIP in P. fijiensis is mostly restricted to repetitive elements rather than genes. 

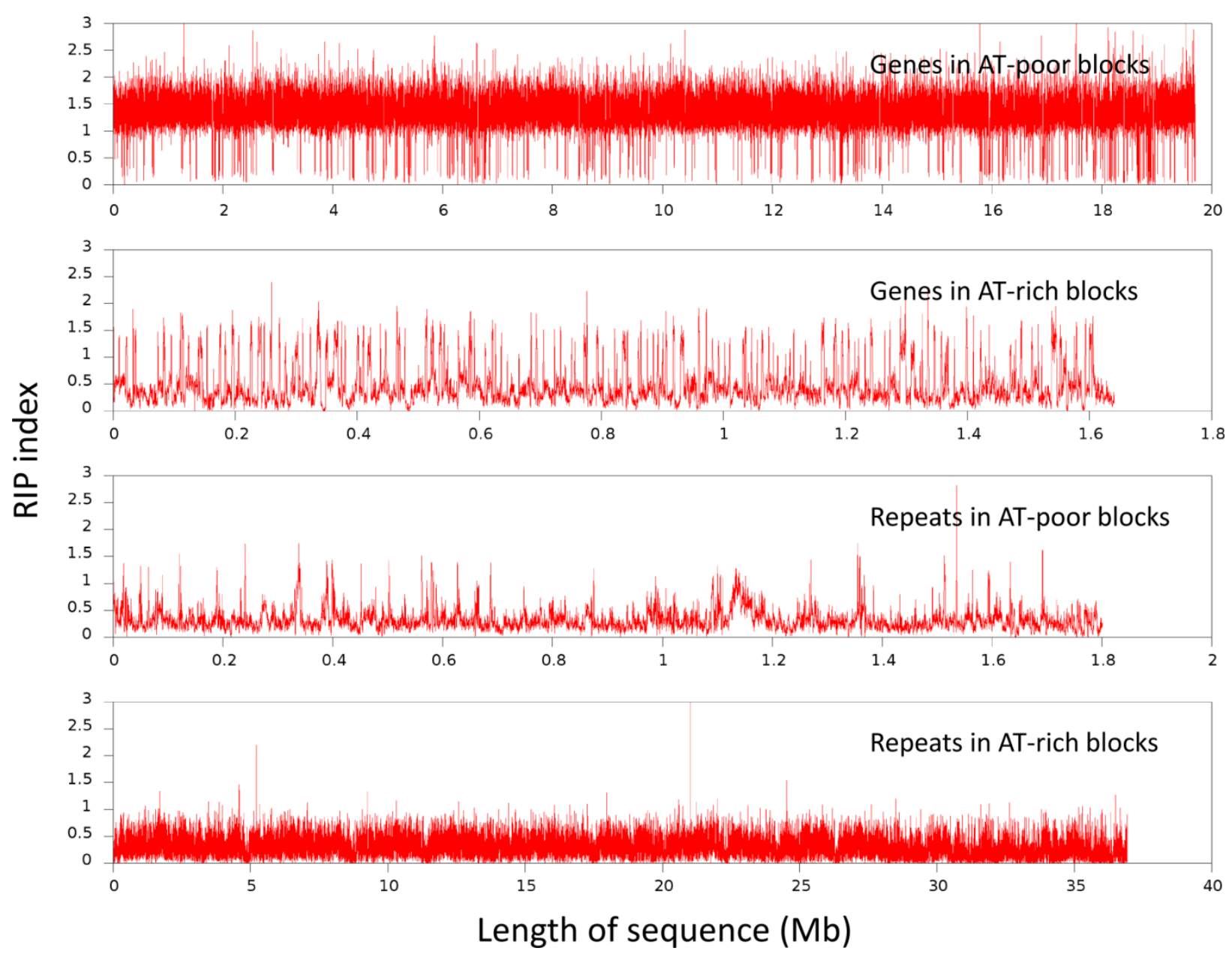

Figure S2. The RIP index in genes and repeats in AT-rich and-poor regions of the $P$. fijiensis genome. RIP is mostly absent from the genes but highly prevalent among the repeated elements of the genome. 


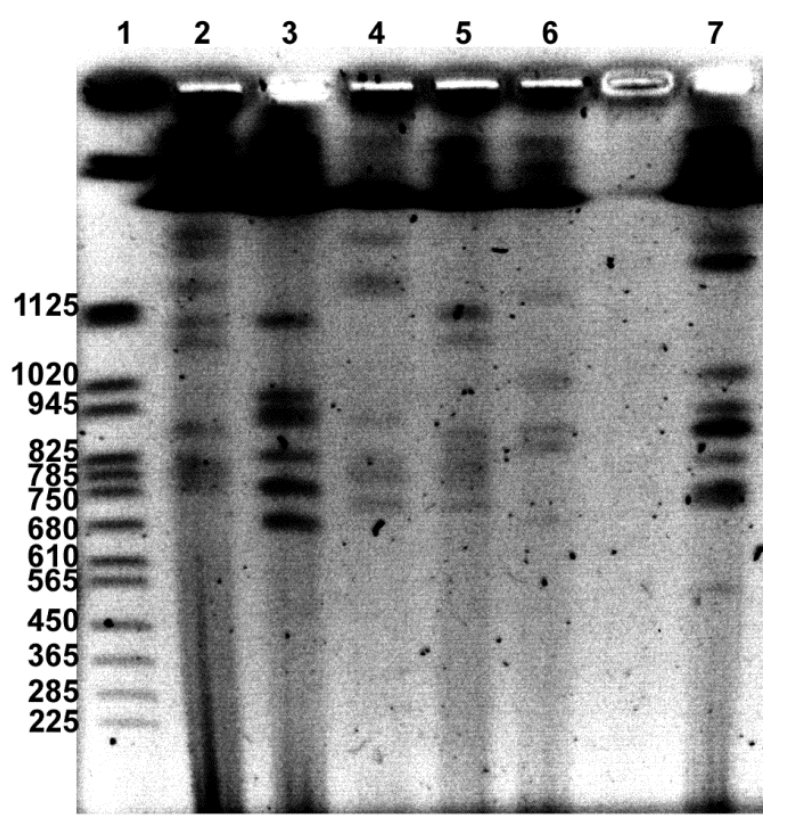

Figure S3. Separation of small size chromosomal bands by electrophoretic karyotyping of five field isolates and the CIRAD139A strain of Pseudocercospora fijiensis. Lane 1, chromosomes from Saccharomyces cerevisiae as high-molecular-weight (HMW) marker; lanes 2 to 6, different field isolates from the Cartagena farm; lane 7, the CIRAD139A strain. Marker sizes are in $\mathrm{Kb}$. 


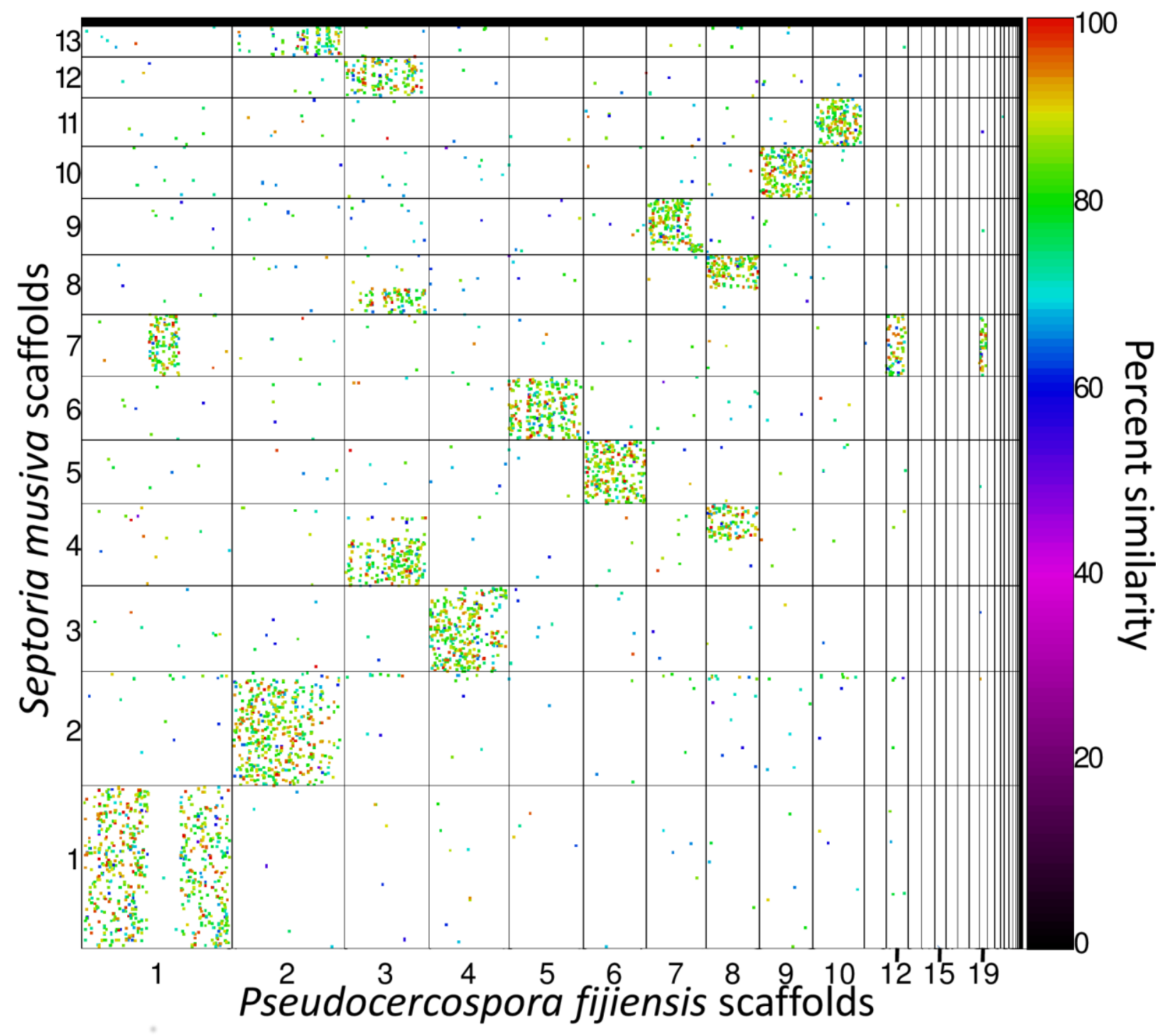

Figure S4. Dot plot showing mesosynteny between the scaffolds of Pseudocercospora fijiensis and Septoria musiva. 


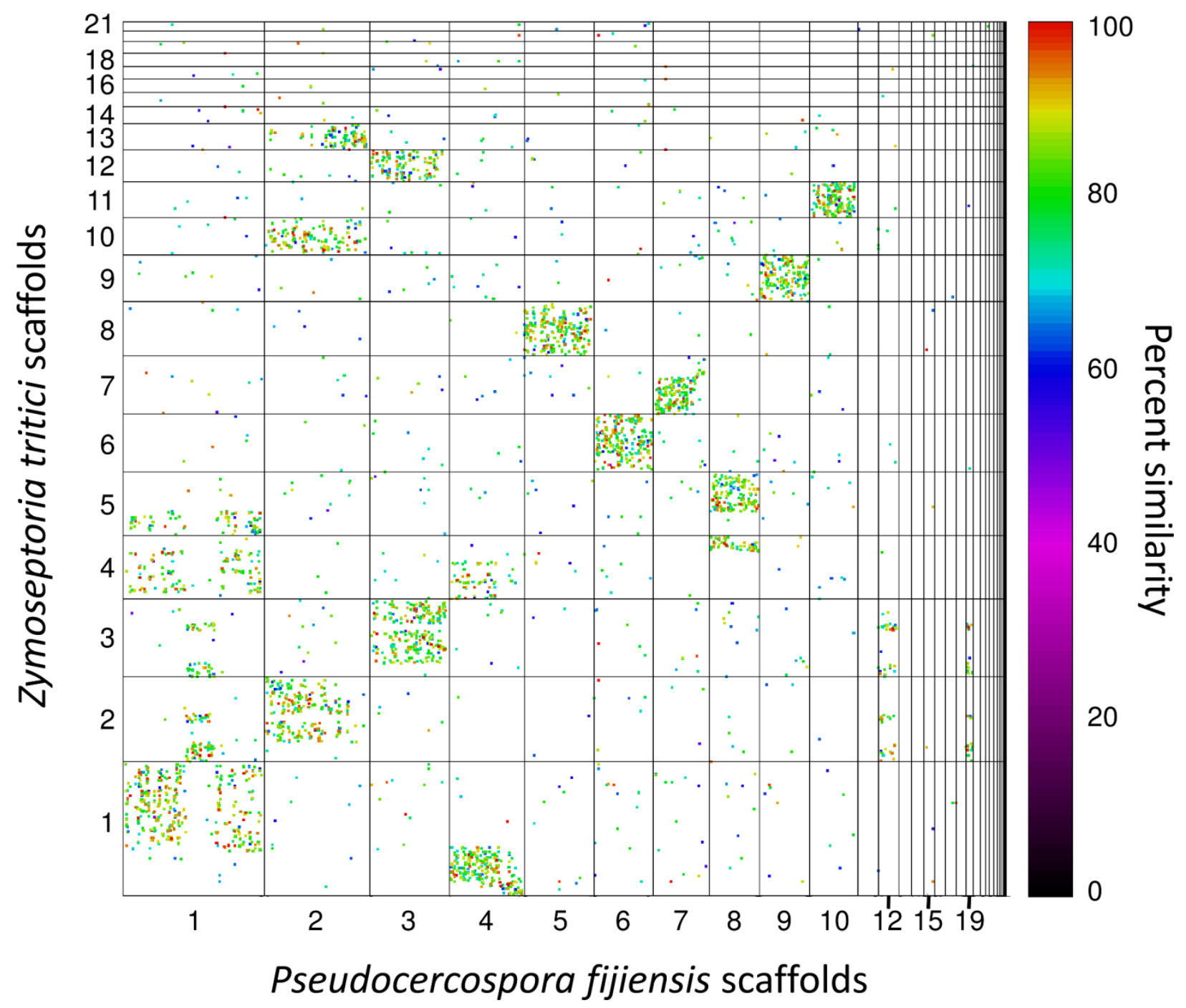

Figure S5. Dot plot showing mesosynteny between the scaffolds of Pseudocercospora fijiensis and Zymoseptoria tritici. 


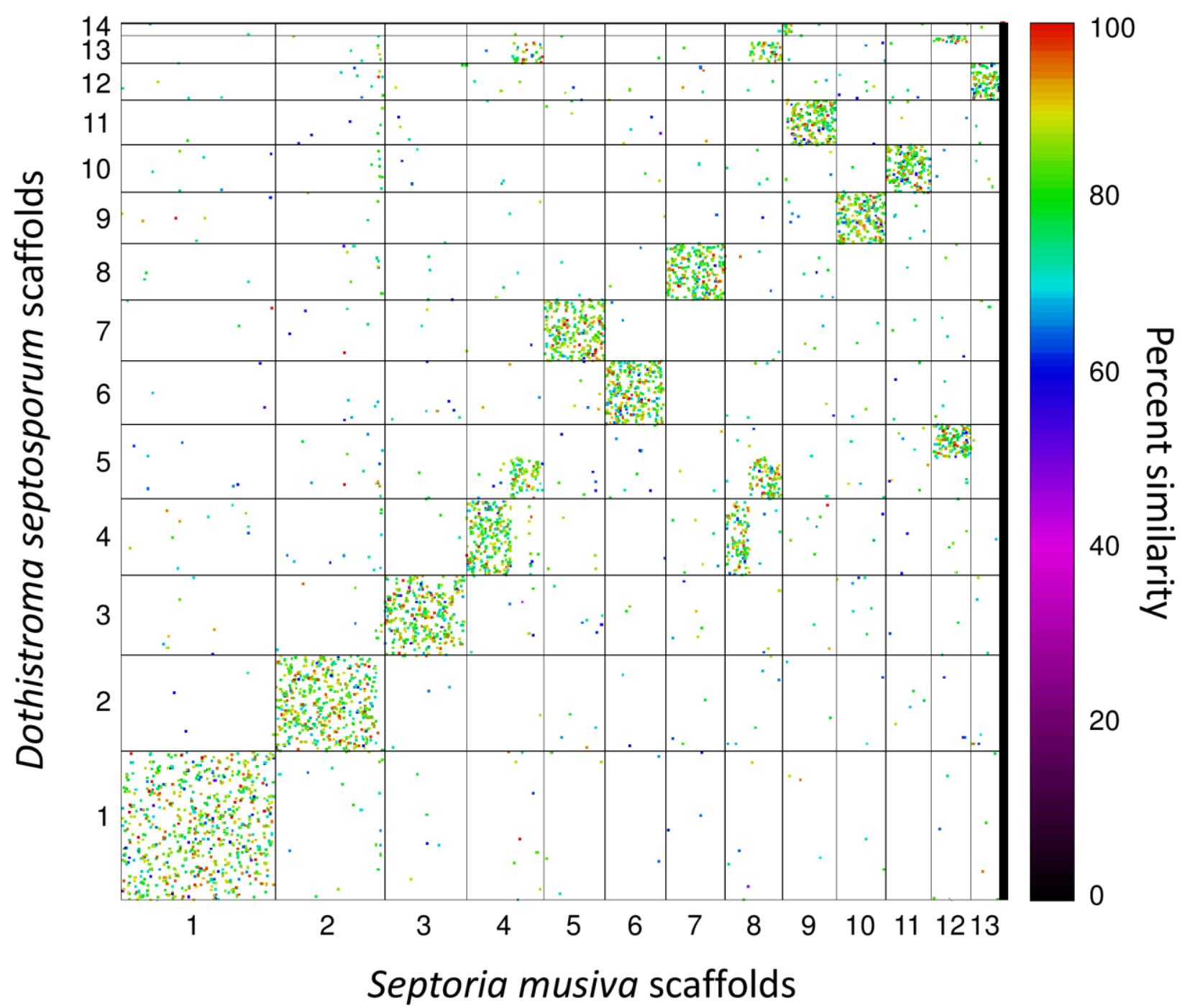

Figure S6. Dot plot showing mesosynteny between the scaffolds of Septoria musiva and Dothistroma septosporum. 


\section{CHAPTER 2}

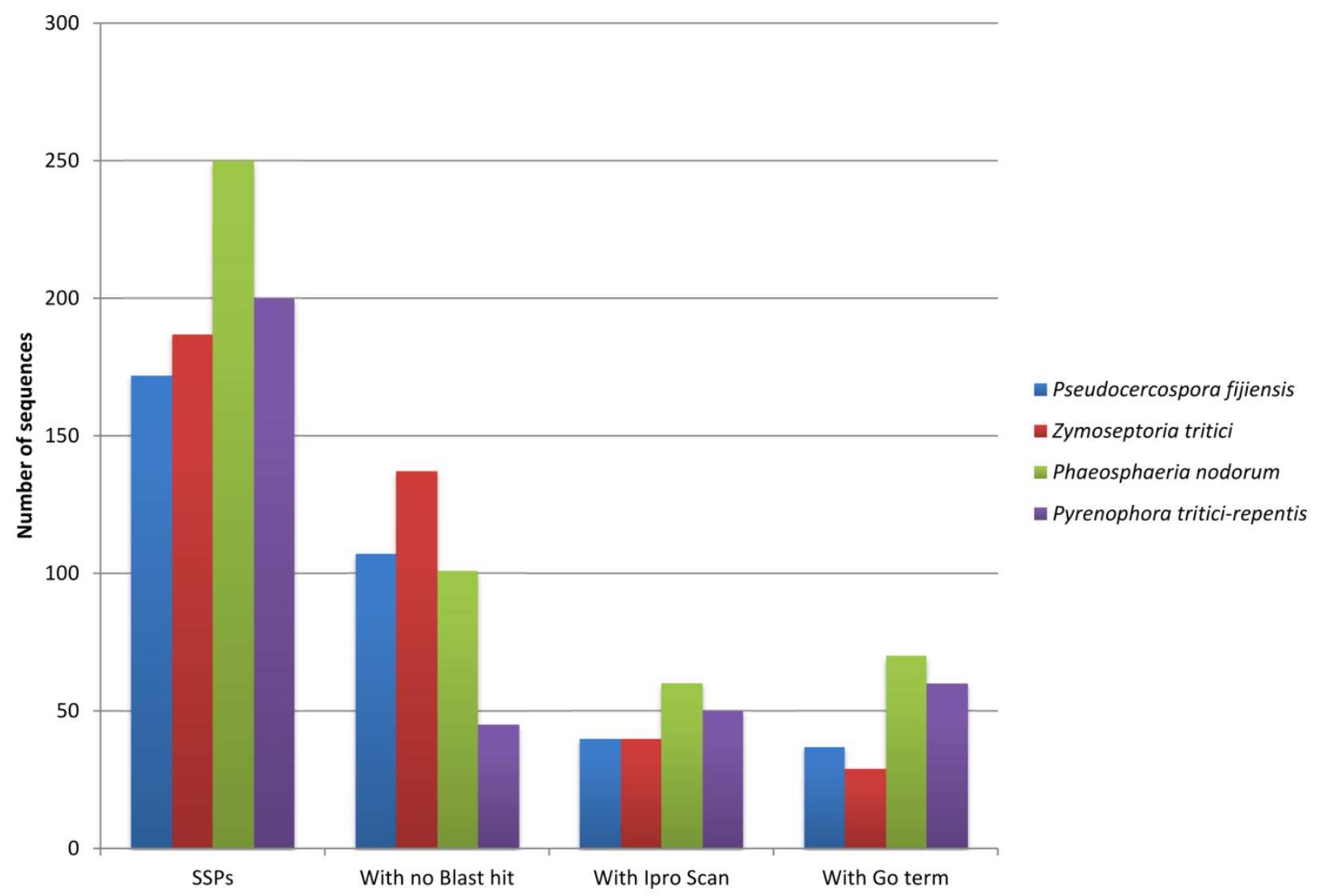

Figure S7. Small secreted proteins in the genome of Pseudocercospora fijiensis compared to those in the genomes of three other Dothideomycetes. 


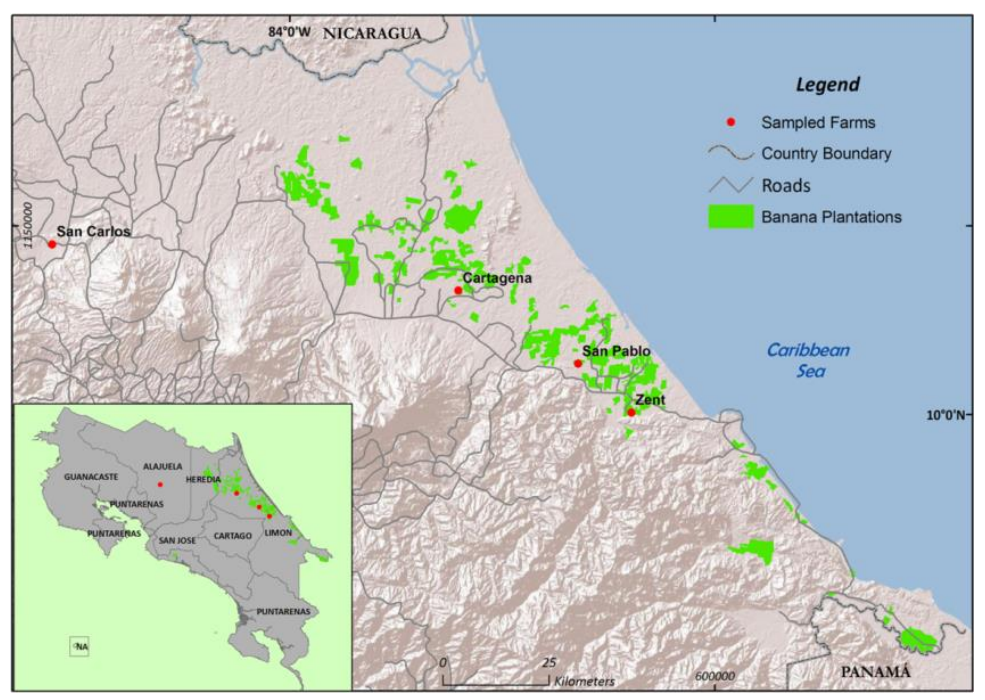

Figure S8. Locations of farms in Costa Rica that were sampled to obtain isolates of Pseudocercospora fijiensis for analyses of mating type, fungicide resistance and population genetics. Farms Cartagena, San Pablo and Zent are in a major banana-production area and are sprayed heavily with fungicides; the San Carlos farm is in an area of plantain production (mostly resistant to $P$. fijiensis) and is not sprayed with fungicides. 
Table S1. Scaffold sizes, and identification of potential dispensable chromosomes in the genome of Pseudocercospora fijiensis by comparison with the characteristics of those known from Zymoseptoria tritici.

\begin{tabular}{|c|c|c|c|c|c|c|c|c|c|}
\hline Scaffold & Size (bp) & $\begin{array}{c}\mathrm{G}+\mathrm{C}, \\
\%\end{array}$ & Genes & Genes/Mb & Genes, $\%$ & $\begin{array}{l}\text { Genes with } \\
\text { PFAM, \% }\end{array}$ & $\begin{array}{l}\text { No. of } \\
\text { ESTs }\end{array}$ & $\begin{array}{l}\text { ESTs, } \\
\%\end{array}$ & $\begin{array}{l}\text { Repeats, } \\
\%\end{array}$ \\
\hline 1 & 11880248 & 46.8 & 2676 & 226.8 & 20.4 & 54.7 & 7277 & 23.00 & 32.6 \\
\hline 2 & 8841024 & 46.2 & 1881 & 214.4 & 14.4 & 52.2 & 4555 & 14.40 & 33.7 \\
\hline 3 & 6657679 & 46.5 & 1526 & 229.8 & 11.6 & 51.0 & 3437 & 10.86 & 31.2 \\
\hline 4 & 6264405 & 45.8 & 1207 & 193.3 & 9.2 & 47.1 & 2725 & 8.61 & 37.0 \\
\hline 5 & 5901819 & 44.8 & 975 & 166.0 & 6.7 & 49.6 & 2149 & 6.79 & 45.2 \\
\hline 6 & 4991523 & 45.0 & 863 & 174.0 & 6.6 & 49.0 & 2413 & 7.63 & 41.7 \\
\hline 7 & 4695110 & 45.7 & 854 & 182.3 & 6.5 & 44.3 & 1953 & 6.17 & 37.6 \\
\hline 8 & 4236865 & 46.2 & 886 & 210.0 & 6.8 & 54.6 & 2030 & 6.42 & 37.4 \\
\hline 9 & 4185348 & 45.2 & 741 & 178.1 & 5.7 & 52.4 & 1297 & 4.10 & 40.1 \\
\hline 10 & 4009308 & 45.4 & 703 & 177.1 & 5.4 & 45.5 & 2842 & 8.98 & 42.1 \\
\hline $11^{*}$ & 1762310 & 40.2 & 62 & 35.3 & 0.5 & 3.2 & 13 & 0.04 & 66.0 \\
\hline 12 & 1674337 & 44.2 & 253 & 151.4 & 1.9 & 47.0 & 539 & 1.70 & 43.6 \\
\hline $13^{*}$ & 1121713 & 41.0 & 51 & 45.5 & 0.4 & 0.0 & & & 63.1 \\
\hline $14^{*}$ & 1006785 & 39.7 & 37 & 36.9 & 0.3 & 0.0 & 6 & 0.02 & 60.1 \\
\hline $15^{*}$ & 928877 & 41.6 & 42 & 45.4 & 0.3 & 4.8 & 13 & 0.04 & 60.8 \\
\hline $16^{*}$ & 905553 & 39.1 & 2 & 2.2 & 0.0 & 0.0 & & & 76.0 \\
\hline $17 *$ & 851953 & 42.1 & 65 & 77.5 & 0.5 & 4.6 & 30 & 0.09 & 32.0 \\
\hline $18^{*}$ & 830772 & 41.7 & 49 & 59.2 & 0.4 & 2.0 & 38 & 0.12 & 51.5 \\
\hline 19 & 609425 & 46.2 & 130 & 213.8 & 1.0 & 45.4 & 255 & 0.81 & 41.4 \\
\hline $20 *$ & 594030 & 41.1 & 29 & 49.1 & 0.2 & 0.0 & 22 & 0.07 & 41.9 \\
\hline $21 *$ & 427043 & 43.6 & 40 & 94.1 & 0.3 & 2.5 & 21 & 0.07 & 49.4 \\
\hline $22 *$ & 366261 & 41.7 & 30 & 82.3 & 0.2 & 3.3 & & & 53.1 \\
\hline $23^{*}$ & 339557 & 37.5 & 0 & 0.0 & 0.0 & 0.0 & & & 66.8 \\
\hline $24 *$ & 325668 & 38.1 & 0 & 0.0 & 0.0 & 0.0 & & & 55.2 \\
\hline $25^{*}$ & 230844 & 42.1 & 1 & 4.4 & 0.0 & 0.0 & & & 12.5 \\
\hline
\end{tabular}

*Potential dispensable chromosomes are characterized by lower gene densities and higher proportions of repetitive sequences. 
Table S2. Comparison of annotated genes in the version 1 and 2 assemblies of the Pseudocercospora fijiensis genome.

\begin{tabular}{lcc}
\hline Annotation parameter & v1.0 Assembly & v2.0 Assembly \\
\hline Number of gene models & 10,316 & 13,107 \\
Gene density per Mb DNA & 140.5 & 187.6 \\
Average gene length & $1629 \mathrm{nt}$ & $1833 \mathrm{nt}$ \\
Average protein length & $436 \mathrm{aa}$ & $427 \mathrm{aa}$ \\
Average exon frequency & 2.45 & 3.62 \\
Average exon length & $578 \mathrm{nt}$ & $395 \mathrm{nt}$ \\
Average intron length & $148 \mathrm{nt}$ & $154 \mathrm{nt}$ \\
Percent complete gene models & 82 & 88 \\
Percent of genes with homology support & 70 & 74 \\
Percent of genes with Pfam domains & 48 & 49 \\
Percent of genes with EST support & & 30 \\
\hline
\end{tabular}

${ }^{\mathrm{a}}$ Number of exons per gene

${ }^{\mathrm{b}}$ With start and stop codons. 
Table S3. Summary of $\mathrm{G}+\mathrm{C}$ contents derived from the melting-curve analyses of DNA extracted from isolates of Pseudocercospora fijiensis, P. eumusae, P. musae and Zymoseptoria tritici.

\begin{tabular}{|c|c|c|c|c|}
\hline \multirow[b]{2}{*}{ Species } & \multirow[b]{2}{*}{ Isolate } & \multicolumn{2}{|c|}{ Percent $\mathrm{G}+\mathrm{C}$} & \multirow{2}{*}{$\begin{array}{l}\text { Number of } \\
\text { replications }\end{array}$} \\
\hline & & Peak 1 & Peak 2 & \\
\hline P. fijiensis & CIRAD86 & $39.4 \pm 0.80$ & $51.6 \pm 0.90$ & 18 \\
\hline P. eumusae & CBS122457 & $39.6 \pm 0.03$ & $51.6 \pm 0.08$ & 2 \\
\hline P. musae & UQ430 & $37.2 \pm 1.30$ & $50.9 \pm 0.50$ & 6 \\
\hline Z. tritici & IPO323 & - & $53.1 \pm 0.50$ & 6 \\
\hline
\end{tabular}


Table S4. Next-generation resequencing information for Pseudocercospora fijiensis isolate CIRAD139A and numbers of Single-Nucleotide Polymorphisms (SNPs) compared with the reference genome of isolate CIRAD86 V2.0.

\begin{tabular}{lr}
\hline \multicolumn{1}{c}{ Parameter } & \multicolumn{1}{c}{ Number $(\%)$} \\
\hline Total reads & $73,185,656$ \\
Uniquely aligned & $43,622,808(59.6 \%)$ \\
Ambiguously mapped & $20,982,018(28.7 \%)$ \\
Unmapped & $8,580,830(11.7 \%)$ \\
Genome coverage (bp) & 514,953 \\
Average depth of coverage & $65,956,770(89 \%)$ \\
Total variants & $509,749(99 \%)$ \\
Total SNPs & $5,204(1 \%)$ \\
Total indels & $95,335(18.5 \%)$ \\
Variants in coding sequences & $46,295(9 \%)$ \\
Non-synonymous variants &
\end{tabular}


Table S5. Analysis of polymorphism type [Single-Nucleotide Polymorphisms (SNPs) and insertion/deletion (indels)] and frequency among the 21 largest scaffolds of the sequenced Pseudocercospora fijiensis isolate CIRAD86 and the re-sequenced isolate CIRAD139A.

\begin{tabular}{lccccc}
\hline Scaffold & $\begin{array}{c}\text { Length } \\
\left(10^{4} \text { bp }\right)\end{array}$ & $\begin{array}{c}\text { Total } \\
\text { variants }\end{array}$ & Substitutions & Indels & $\begin{array}{c}\text { Mean } \\
\text { frequency } \\
\text { (per 10kb) }\end{array}$ \\
\hline 1 & 1188 & 71940 & 70994 & 946 & 60.6 \\
2 & 885 & 58469 & 57793 & 676 & 66.1 \\
3 & 667 & 44949 & 44352 & 597 & 67.4 \\
4 & 627 & 45844 & 45299 & 545 & 73.1 \\
5 & 591 & 41410 & 41033 & 377 & 70.1 \\
6 & 500 & 37467 & 37088 & 379 & 74.9 \\
7 & 470 & 36909 & 36542 & 367 & 78.5 \\
8 & 425 & 28845 & 28473 & 372 & 67.9 \\
9 & 420 & 28315 & 28021 & 294 & 67.4 \\
10 & 402 & 28985 & 28707 & 278 & 72.1 \\
11 & 177 & 14887 & 14833 & 54 & 84.1 \\
12 & 168 & 12317 & 12204 & 113 & 73.3 \\
13 & 113 & 6695 & 6691 & 4 & 59.2 \\
14 & 102 & 8049 & 8027 & 22 & 78.9 \\
15 & 94 & 6700 & 6668 & 32 & 71.3 \\
16 & 91 & 6292 & 6277 & 15 & 69.1 \\
17 & 86 & 6301 & 6281 & 20 & 73.3 \\
18 & 84 & 6155 & 6130 & 25 & 73.3 \\
19 & 62 & 3781 & 3743 & 38 & 61.0 \\
20 & 60 & 4235 & 4234 & 1 & 70.6 \\
21 & 44 & 3517 & 3492 & 25 & 79.9 \\
\hline
\end{tabular}


Table S6. Genotyping strobilurin resistance and mating type in four Costa Rican populations of Pseudocercospora fijiensis.

\begin{tabular}{|c|c|c|c|c|c|c|c|}
\hline \multirow[b]{2}{*}{ Population } & \multirow[b]{2}{*}{$\begin{array}{c}\text { Sample } \\
\text { size }^{\mathrm{a}}\end{array}$} & \multicolumn{3}{|c|}{ Fungicide sensitivity } & \multicolumn{3}{|c|}{ Mating type } \\
\hline & & Sensitive & Resistant & $\begin{array}{l}\text { Percent } \\
\text { resistant }\end{array}$ & Ratio & $\chi^{2}$ & $P^{\mathrm{b}}$ \\
\hline Cartagena & 189 & 0 & 178 & 100.0 & $90: 85$ & 0.14 & 0.705 \\
\hline San Carlos & 91 & 87 & 0 & 0.0 & $42: 45$ & 0.10 & 0.748 \\
\hline San Pablo & 179 & 0 & 180 & 100.0 & $84: 82$ & 0.02 & 0.877 \\
\hline Zent & 190 & 14 & 162 & 92.0 & $77: 96$ & 2.09 & 0.149 \\
\hline Total or mean & 649 & 101 & 520 & 83.7 & $293: 308$ & 0.37 & 0.541 \\
\hline
\end{tabular}

${ }^{\mathrm{a}}$ Total number of isolates with molecular marker data for at least one locus. Sample sizes for each statistic varied depending on the number of isolates scored successfully.

${ }^{\mathrm{b}}$ Probability that the observed mating type ratios are not significantly different from 1:1. 
Table S7. Estimates of genetic differentiation ${ }^{\mathrm{a}}$ (Jost's D above the diagonal and the corrected, standardized genetic differentiation $\mathrm{G}^{\prime \prime}$ Sт below the diagonal) between pairs of populations of Pseudocercospora fijiensis sampled from four banana plantations in Costa Rica ${ }^{\mathrm{b}}$.

\begin{tabular}{lllll}
\hline Population & Cartagena & San Carlos & San Pablo & Zent \\
\hline Cartagena & $-^{\mathrm{c}}$ & 0.067 & 0.052 & 0.089 \\
San Carlos & 0.067 & - & 0.192 & 0.126 \\
San Pablo & 0.116 & 0.097 & - & 0.065 \\
Zent & 0.190 & 0.243 & 0.146 & - \\
\hline
\end{tabular}

a $D$ and $G^{\prime \prime}{ }_{S T}$ were calculated with GenoDive (Meirmans and van Tienderen, 2004) as described by Jost (2008) and Meirmans and Hedrick (2011), respectively.

${ }^{\mathrm{b}}$ All values for each statistic were significantly different at $P=0.001$.

${ }^{\mathrm{c}}$ Not applicable to self comparisons. 
Table S8. Percent of gene models supported by different kinds of evidence in the initial automated annotation of the genome of Pseudocercospora fijiensis.

\section{Annotation parameter Number or percent of genes}

\begin{tabular}{lc}
\hline Number of gene models & 13,107 \\
Percent complete (with start and stop codons) & $88 \%$ \\
Percent of genes with homology support & $74 \%$ \\
Percent of genes with Pfam domains & $49 \%$ \\
Percent of genes with EST support & $30 \%$ \\
\hline
\end{tabular}


Table S9. Automated assignment of the 13,107 genes in the genome of Pseudocercospora fijiensis to broad functional categories.

\begin{tabular}{lc}
\hline \multicolumn{1}{c}{ Category } & Number of genes (Percent) \\
\hline Proteins assigned to a KOG & $6676(51 \%)$ \\
KOG categories genome-wide & 3247 \\
Proteins assigned a GO term & $5655(43 \%)$ \\
GO terms genome-wide & 1393 \\
Proteins assigned an EC number & $2666(20 \%)$ \\
EC numbers genome-wide & 798 \\
Proteins assigned a Pfam domain & $6603(50 \%)$ \\
Pfam domains genome wide & 2446 \\
\hline
\end{tabular}


Table S10. Primers used for population genetic diversity and fungicide sensitivity assays in four Pseudocercospora fijiensis populations in Costa Rica.

\begin{tabular}{cllc}
\hline Locus & Forward primer $^{\mathrm{a}}$ & Reverse primer $^{\mathrm{a}}$ & $\begin{array}{l}\text { Expected } \\
\text { size }\end{array}$ \\
\hline Fungicide sensitivity & & \\
$c y t b$ & CTCAAATACTGCCTCAGC & CCGTAATGTGGTTCATC & 285 \\
& CTCAAATACTGCCTCAGC & GTTATAACTGTAGCTCC & 198 \\
& & & \\
Variable Number of Tandem repeats (VNTR) loci & & \\
1333 & GAGTGAAGTACTGCGGAGGC & AGTTGGAGAAAGGCGAAAGG & 259 \\
3959 & GCGCGAGGCTTTCTATCTC & ACCCCGATTAGGGAAGGTC & 184 \\
3786 & GCAGCGGAGTGCTAGTAACC & CGCGCTTTTGACTCTTCTTC & 260 \\
0252 & TAGAGGCTACCCTGCCGTC & GTATACTTCCGACCTCGGGC & 132 \\
0705 & ATAGGATGCGGCAGACACTC & CGTCGCGATTTGAAGTGCC & 214 \\
\hline
\end{tabular}

${ }^{\text {a }}$ Primer sequences written 5' to 3 '. 
CHAPTER 2 


\section{CHAPTER 3}

\section{TARGETED AND RANDOM GENETIC MODIFITATION OF THE BLACK SIGATOKA \\ PATHOGEN PSEUDOCERCOSPORA FIJIENSIS BY AGROBACTERIUM TUMEFACIENS - MEDIATED TRANSFORMATION}

Caucasella Díaz Trujillo, Adilson Kobayashi, Manoel Souza, Pablo Chong, Harold J. G. Meijer, Rafael Arango Isaza and Gert H. J. Kema. To be submitted. 


\section{Abstract}

Pseudocercospora fijiensis (syn. Mycosphaerella fijiensis) is the fungal pathogen that causes black leaf streak disease or black Sigatoka in banana and plantain crops, which is a threat to global banana production, and unfortunately the experimental amenability of the fungus is low as it develops very slowly, hardly produces conidia and molecular tools for functional genetics are barely available. Here, we report successful Agrobacterium tumefaciens-mediated transformation and the generation of various genetically modified $P$. fijiensis strains. By random insertion we developed green fluorescent protein (GFP) and red fluorescent protein (DsRed) labelled strains. Through homologous recombination we also generated the first $P$. fijiensis Pfavr4 and Pfku70 knock-out mutants. The former is the first avirulence gene in this pathosystem recognized by the cognate tomato resistance gene $C f 4$. The latter gene encodes a protein that triggers non-homologous end joining. Likewise, we generated various $P$. fijiensis strains with the $P f c y p 51$ promoter replaced. This gene encodes CYP51, and its encoded protein is the target for lanosterol $14 \alpha$-demethylase inhibitors (DMIs; azoles), a major class of functional fungicides for black Sigatoka control in banana and also applied against many other fungal plant, animal and human pathogens. With the targeted strains obtained here, we developed tools to further analyze the $P$. fijiensis-banana interaction, and to better understand reduced sensitivity to azole fungicides. 


\section{Introduction}

Banana and plantain are major fruit and staple crops (FAO, 2015). Bananas are the global top export fruit, with an average of 114 million tons/year, dominated by Cavendish clones that take a major share (90\%), but which are also increasingly important for local markets (FAO, 2003; Food and Agriculture Organization of the United Nations, 2015). Cooking bananas and plantains are major staple foods in tropical and subtropical countries in central Africa, South East Asia and Latin America (Mohan and Swennen, 2004; Ortiz and Swennen, 2014). Many banana varieties are highly susceptible to black Sigatoka or black leaf streak disease (Fullerton and Olsen, 1995). Disease management in this perennial crop largely relies on frequent fungicide applications. The frequency varies over environments but is commonly over 50 times per year (Chong, 2016; De Lepeyre De Bellaire 2010; FRAC, 2010) and commonly represents up to $40 \%$ of the total production costs (Chong, 2016; MartínezBolaños et al., 2012). Albeit that banana production provides manifold jobs and supports the livelihoods of millions of people, the dependency on fungicides evidently threatens occupational health as well as tropical fragile environments (Panganiban et al., 2004; Penagos, 2002).

Pseudocercospora fijiensis (Morelet, 1969) Deighton (1976), previously known as Mycosphaerella fijiensis (Morelet 1969) is the causal agent of black Sigatoka and is a hemibiotrophic ascomycete fungus from the class of the Dothideomycetes (Schoch et al., 2006), which has spread to almost all tropical and subtropical regions (Carlier et al., 1996; Hayden and Carlier, 2003; Rivas et al., 2004). The life cycle of the pathogen includes a biotrophic and a necrotrophic stage, that eventually results in the production of clonal conidia and genetically diverse sexual ascospores that drive epidemics (Burt, 1994). Once germinated, germ tubes of conidia and ascospores penetrate the leaf through the stomata and develop into mycelia that colonize the mesophyll tissue until fructification. During this process lesions of 
affected foliar tissue become gradually visible and eventually coalesce in large necrotic blotches along the rim of the leaf towards the midrib. Clearly, leaf necrosis reduces the photosynthetic capacity, but also causes a metabolic switch to premature fruit ripening, which greatly affects the export market value of the crop (Beltrán-García et al., 2014; Okole and Schulz, 1997).

The genome analysis of $P$. fijiensis (Arango Isaza et al., 2016) generated a range of genetic studies (Chang et al., 2016; Chong, 2016; Churchill, 2011; Couoh-Uicab et al., 2013; de Wit et al., 2012; Kantún-Moreno et al., 2013; Noar and Daub, 2016a, 2016b; Ohm et al., 2012; Stergiopoulos et al., 2014, 2010), which turned the fungus from a recalcitrant pathogen into an interesting pathogen with unique characteristics. It has for instance one of the largest genomes in the Dothideomycetes due to a huge transposon driven genome expansion (Ohm et al., 2012). Hence, $P$. fijiensis is not only important as a plant pathogen, but is also attractive for genome wide comparative analyses (Chang et al., 2016). Nevertheless, the genetic amenability of the fungus is limited. It has a slow in vitro growth, poor sporulation that largely depends on isolate and environmental conditions, can only complete a sexual cycle in planta, and foremost, lacked a transformation protocol for a long time, which hampered any functional analysis of genes (Arango et al., 2016; Churchill, 2011).

Agrobacterium tumefaciens mediated transformation (ATMT) has been successfully used in many filamentous fungi (Abello et al., 2008; Chen et al., 2000; Combier et al., 2003; de Boer et al., 2013; Ding et al., 2011; Mirzadi Gohari et al., 2014; Nyilasi et al., 2005; Weld et al., 2006; Xue et al., 2013; Zheng et al., 2011; Zwiers and De Waard, 2001). Homologous recombination frequencies vary between 1 and 10\% (Kück and Hoff, 2010), depending on the fungus and the targeted genes. Nevertheless, knocking out $k u 70$ genes, that encode a protein involved the DNA non-homologous end joining (NHEJ) for double-strand DNA break repair, has greatly contributed to enhanced recombination in various fungi (Bowler, et al., 2010; 
Feng, et al., 2012; He et al., 2013; Nayak et al., 2006; Ninomiya, et al.,2004).

Here, we report the Agrobacterium tumefaciens mediated transformation (ATMT) of P. fijiensis. Because the in vitro production of P. fijiensis conidia is challenging (Churchill, 2011; Cruz-Martín et al., 2011;; Donzelli and Churchill, 2007), our approach involves mycelium for ATMT and this resulted in the production of several transformed $P$. fijiensis strains with ectopic integrations as well as homologous recombinations that can be used for detailed pathological and functional analyses.

\section{Results}

Random mutagenesis: generation and characterization of gfp and DsRed Pseudocercospora fijiensis mutants

Growth inhibition tests revealed that a hygromycin concentration of $50 \mu \mathrm{g} \cdot \mathrm{mL}^{-1}$ enabled the differentiation of transformed from untransformed colonies of the CIRAD86 and CIRAD139a wt $P$. fijiensis recipient strains (data not shown). Random integration of the generated $g f p$ or DsRed constructs in the $P$. fijiensis recipient strains (Figure 1) was routinely checked through fluorescence microscopy. In three independent transformation experiments we obtained an average of 9.125 transformants per membrane by starting with a concentration of $1.5 \times 10^{6}$ hyphal pieces per $\mathrm{mL}$ and eventually obtained each combination of fluorescent marker and recipient strain viz. strains CIRAD86::gfp, CIRAD86::DsRed, CIRAD139a::gfp and CIRAD139a::DsRed, with transformation efficiency of $0.00066 \%, 0.0011 \%, 0.0002 \%$, and $0.00006 \%$, respectively (Table 1); and whose genetic identities were confirmed using a genetic profile of repetitive DNA at different loci by using the Variable Number of Tandem Repeat markers (VNTR1333; Garcia et al., 2010) (Figure 2). We did not observe in vitro obvious morphological differences between the wt and mutant strains on solid growth medium. 
Targeted mutagenesis: generation and identification of Pfavr4, Pfku70 knock-out strains and Pfcyp51 promoter replacement

The homolog of Cladosporium fulvum Avr4 in P. fijiensis is Pfavr4 (Stergiopoulos et al., 2010, 2014) and is located (ID: 87167) on position 183210-183796 of scaffold 4. For the knock-out construct, we selected the upstream region 180469-183065 and the downstream region 183458-185053 (Figure 1). The $P f k u 70$ gene was identified from the $P$. fijiensis genome database (Arango Isaza et al., 2016) and is located (ID: 65414) on scaffold 3, position 603346-605427. This includes the start codon $21 \mathrm{bp}$ upstream of the automatically predicted transcript. Note that the transcript was extended at the N-terminus without addition of introns. The resulting protein model of 702 aa is in agreement with KU70 homologs from other fungi, including Sphaerulina musiva (EMF13337.1), P. musae (KXT17510.1), and Zymoseptoria brevis (KJY00058.1) which have similar extensions. Aligning the KU70 proteins revealed that the $P$. fijiensis protein is the closest related to the Dothideomycete fungi $S$. musiva (74.2 $\%)$ and Z. tritici (69.9\%) (Figure 2), whereas the identity with the more distantly related basidiomycete Rhodosporium toruloides (AIA21643.1) was significantly lower (39.6\%) (Figure 3; Table 2). Domain analysis confirmed the presence of three domains, similar to other eukaryotic KU70 proteins (Figure 3): the $\mathrm{N}$-terminus with a $\mathrm{Ku} \_\mathrm{N}$ terminal $\alpha / \beta$ domain (also called von Willebrand A [vWA] domain), in the center a KU core domain including a DNA-binding $\beta$-barrel domain, and finally the C-terminus comprising a SAP domain (Fell and Schild-Poulter, 2015) (Figure 3A).

Using LBA1100 Agrobacterium tumefaciens cells, we obtained 90 and 102 GFP fluorescent transformants for Pfavr4 and Pfku70, respectively, which were tested by PCR for homologous recombination. Ectopic transformants showed the same PCR fragment as the CIRAD86 wt strains, whereas replacement mutants lacking this amplicon were positive for the strain specific variable number tandem repeat marker VNTR1333. Moreover, the presence 
of the replacement cassette was confirmed by amplification of a diagnostic amplicon with a primer located within the replacement cassette and a unique sequence of the $P$. fijiensis genome outside the construct (Figures 5 and 6). Overall, these rounds of transformations resulted in the identification of three knock-out mutants: Pfavr4 \#10, Pfku70 \#33 and Pfku70 \#45.

By using the A. tumefaciens AGL1 strain in combination with the modifications in the protocol after the initially random mutagenesis, we improved the transformation efficiency with at least one order of magnitude for Pfku70 (two knock out strains out of 102 tested hygromycin transformants, derived from $4.1 \times 10^{5}$ hyphal fragments per $\mathrm{mL}$ ). This resulted in an overall transformation efficiency of $0.025 \%$ and homologous recombination in $1.96 \%$ across all transformants. The knock-out efficiency for PfAvr4, using the same conditions, was $0.022 \%$ and homologous recombinations occurred in $1.1 \%$ of the transformed strains (Table $1)$.

The third sequence we targeted using this method was the Pfcyp51 gene promoter (Chong et al., 2016). With a transformation efficiency of $0.025 \%$, we replaced the promoter of the azole sensitive strain E22 by the promoter of the azole resistant strain Ca5_16, which carried no and multiple repetitive elements in their promoters, respectively. Additional to promoter length, a diagnostic amplicon discerned two homologous recombinants resulting in a recombination efficiency of $0.8 \%$ (Table 1$)$.

Fitness comparisons between the wt strain CIRAD86 and the Pfku70\#33 and Pfku70\#45 strains showed that the knock-out strains were compromised in growth (-25-38\%) (Figure 7). However, functional characterization of the $P f c y p 51$ promoter replacement strains did not show significant fitness penalties potentially resulting from the transformation process (Díaz-Trujillo et al., 2018). 


\section{Discussion}

Food security requires continuous crop improvement to meet future demands. The regular and worrisome disease threats of major crops necessitate analyses of the widest genetic resources to identify new genes to increase genetic diversity for all traits contributing to increase productivity. Therefore, it is essential to uncover the potential of such resources by using genetic and phenotypic characterization technologies during abiotic and biotic challenges. Understanding host-pathogen interactions and dynamics is a fundamental basis for sustainable crop health (De Wit, 2016; Kema et al., 2018; 2016; Michelmore et al., 2017; Mirzadi et al., 2015; FRAC, 2010; van den Bosh et al., 2014). For many crops, tools have been developed for the analysis of plant-pathogen interactions, but these are in their infancy for under-investigated crops. Banana is one of these crops, albeit a major staple food for millions of people and despite its status as the top global fruit (FAO, 2015). Disease control is mostly accomplished by extensive fungicide applications due to the vulnerability of the global Cavendish banana monoculture (Diaz-Trujillo et al., 2018). Here, we report the first Agrobacterium-mediated transformation of $P$. fijiensis, which is an important step to aid mining the genome of this major plant pathogen (Arango et al., 2016). We initially applied ATMT for ectopic integration of the DsRed or $g f p$ gene, respectively, and then accomplished targeted mutagenesis by generating knock-out strains of Pfku70, Pfavr4 and replacing the Pfcyp51 promoter. In many fungi $\Delta \mathrm{ku} 70$ strains have shown a significant increase of homologous recombination (Bowler et al., 2010; Catalano et al., 2010; He et al., 2013; Koh et al., 2014; Näätsaari et al., 2014; Ninomiya et al., 2004), and hence we considered it important to develop the $\triangle P f k u 70 P$. fijiensis strain to facilitate further functional analyses of $P$. fijiensis genes. We disrupted Pfavr4, the first effector described in this pathogen, as a starting point to further unravel the banana - P. fijiensis interaction (Arango et al. 2016). Recently, we also 
engineered $P$. fijiensis strains with modification of Pfcyp51 promoters, to identify a new mechanism of fungicide sensitivity (Diaz-Trujillo et al., 2018).

Frequently, conidiospores are reported as the ideal starting material for ATMT (Liu et al., 2009; Nizam et al., 2010; Zwiers and De Waard, 2001), but others have used other tissues (Eckert et al., 2005), and reported higher transformation efficiencies with mycelial pieces (Abello et al., 2008) or even fructifications (Chen et al., 2000). In the case of Hebeloma cylindrosporum (Combier et al., 2003) and Muscodor albus (Ezra et al., 2010), transformation was exclusively possible with mycelial starting material. In $P$. fijiensis, efficient and constant production of conidiospores is challenging. It is strongly influenced by light conditions (Sepúlveda et al., 2009), temperature (Churchill, 2011; Sepúlveda et al., 2009), isolate origin (Peraza-Echeverría et al., 2008), harvesting intervals (Peraza-Echeverría et al., 2008), and the number of subcultures (Cruz-Martín et al., 2011). Therefore, Donzelli et al. (2007) successfully used mycelial fragments instead of conidia to study the banana- $P$. fijiensis interaction. Therefore, we eventually used hyphal material as starting material for ATMT and showed it can be used for functional analysis of at least three genes in this recalcitrant fungus.

The constructed replacement cassette was functional in P. fijiensis. The $h p h$ gene, that confers resistance to hygromycin $\mathrm{B}$, in combination with the trpC terminator, has been widely used in Ascomycetes and other fungi as selection marker (Fitzgerald et al., 2003; Krappmann et al., 2006; Mirzadi Gohari et al., 2014). Binary vectors for fungal transformation as pPm43GW have been developed based on the pCAMBIA vector series and others mainly for plant transformation (Frandsen, 2011). Rather than the classic laborious method to construct the binary vector for gene replacement, we used the simplified and high throughput Gateway cloning system according to Shafran et al. (2008), essential to allow screening of the large number of identified sequences that are potentially involved in pathogenicity, fungal growth and resistance to fungicides (Amil et al., 2007; Arango Isaza et al., 2016; Beltrán-García et 
al., 2014; Cañas-Gutiérrez et al., 2006; Chang et al., 2016; Couoh-Uicab et al., 2013; EscobarTovar et al., 2015; Kantún-Moreno et al., 2013; Noar and Daub, 2016a, 2016b).

Gene knock-out efficiencies depend largely on the length of homologous sequence and is usually optimal at around $1 \mathrm{~kb}$ (Michielse et al., 2005). Low homologous transformation efficiency necessitates the screening of a large number of transformants to identify those with correct integration. A strategy to increase homologous recombination efficiency in fungi is based on the stimulated expression of genes involved in recombination although this is also prone to decreased genomic stability (Natsume et al., 2004). Alternatively, disruption of genes involved in NHEJ was shown to increase the homologous recombination frequency in several filamentous fungi (Bowler et al., 2010; Koh et al., 2014; Krappmann et al., 2006; Ninomiya et al., 2004). For Venturia inaequalis mycelium the observed efficiency was only $0.002 \%$ (Fitzgerald et al., 2003) and it was $0.0026 \%$ for chopped M. albus mycelia (Ezra et al., 2010). For $P$. fijiensis using small young hyphal fragments, we achieved around $0.02 \%$ transformation efficiency. Previously, the transformation efficiencies of P. fijiensis were 0.5-4 (Balint-Kurti et al., 2001) or 2.5-3 (Portal et al., 2012) transformants per $\mu$ g plasmid DNA in a restriction enzyme-mediated integration approach with 80 generated transformants (Portal et al., 2012). It is difficult to compare the efficiency of ATMT with previous $P$. fijiensis transformations, as frequencies are presented in terms of mycelial pieces or $\mu \mathrm{g}$ of plasmid DNA, respectively. More recently, Escobar et al. (2015) obtained $0.12 \%$ transformation efficiency generating random integration mutants using shock waves on $P$. fijiensis conidiospores and by RNAi silenced Pfhogl (Onyilo et al., 2017), which adds another tool for functional assays in this fungus. Whereas homologous recombination efficiency is highly variable from $<1 \%$ to $>80 \%$ depending on the fungal species, isolate, tissue, target gene, and its position on the chromosome and chromatin structure (Kück and Hoff, 2010; Weyda et al., 2017), we obtained a recombination frequency of $1.96 \%$ in $P$. fijiensis by using mycelial 
fragments. Both ectopic transformation and homologous recombination efficiencies in $P$. fijiensis are thus within the same range -although at a low level- as reported for other fungi. This promises good opportunities for future research, even though fine tuning is necessary. Experience learned however, that such efficiencies can be significantly improved once more people start working on a system. Initial ATMT transformation efficiency in Z. tritici yeastlike spores was $0.00007 \%$ (Zwiers and De Waard, 2001) and variable homologous recombination efficiency could be as low as $0.35 \%$ (Cousin et al., 2006). However, Z. tritici is currently considered as a model with constant efficiencies that are two orders of magnitude higher around $0.001 \%$, (Mirzadi Gohari et al., 2014) and homologous recombination as high as $85 \%$ (Bowler et al., 2010). Moreover, the generated $\triangle P f k u 70$ strains might be used for optimizing targeted mutagenesis since this is a key component in the NHEJ machinery. Currently CRISPR/Cas9 for targeted mutagenesis is increasingly being applied to filamentous fungi (Liu et al., 2015; Nødvig et al., 2015; Wenderoth et al., 2017; Weyda et al., 2017) and this also relies on homologous recombination. Consequently the $\triangle P f k u 70$ strains might be valuable starting material for application in P. fijiensis, as in other organisms (Chu et al., 2015).

The $P f k u 70$ gene was identified in the sequenced strain CIRAD86 (Arango Isaza et al., 2016) and is highly conserved when compared to orthologues from other organisms. The encoded protein shared a high identity with KU70 proteins from other Dothideomycetes, indicating a functional conservation and the involvement in NHEJ. A growth reduction was observed in the $P f k u 70$ strains and its function should be accessed in further studies. This was not due to the transformation process since other generated knock-out strains were not affected in fitness and so these were used as a basis for swapping promoter domains of the Pfcyp51 gene (Diaz-Trujillo et al., 2018). In Penicillium chrysogenum, Hoff (2010) showed that genes related to stress response were upregulated in $k u 70$ disruptant strains. In several 


\section{CHAPTER 3}

fungi such as Botrytis cinerea (Choquer et al., 2008), Parastagonospora nodorum (Feng et al., 2012), P. decumbes (Z.-H. Li et al., 2010), M. ruber (He et al., 2013) and Aspergillus parasiticus (Chang, 2008) growth and morphology was not related to the lack of $k u 70$. Slightly retarded growth and upregulated telomere action was detected in $K u 70$ knock-out strains of Candida albicans (Chico et al., 2011). Bowler et al., (2010) did not detect any defects in ku70 mutants of Z. tritici, whereas recently Wu et al., (2017) related NHEJ to transposon insertions as cause of intron mobility. A higher sensitivity to DNA damage was reported in Neurospora crassa (Ninomiya et al., 2004), Pichia pastoris (Näätsaari et al., 2012) and $R$. toruloides (Koh et al., 2014) $\Delta k u 70$ strains. In all these cases higher homologous recombination frequencies were observed by using of $\Delta k u 70$ strains for transformation.

In summary, these data provide a starting point for upscaling functional analyses in $P$. fijiensis. This is important for increased understanding of the P. fijiensis-banana pathosystem and eventually for contributing to improved disease control and the identification of crucial genes for its biology and those that drive pathogenesis. 


\section{Materials and Methods}

\section{Strains}

The sequenced $P$. fijiensis strains CIRAD86 and CIRAD139a (Arango Isaza et al., 2016) were used as wild-type (wt) recipient strains throughout this study. They were grown on potato dextrose agar (PDA) plates amended with streptomycin $100 \mu \mathrm{g} \cdot \mathrm{mL}^{-1}$ for $12-15$ days and were maintained in an incubator (Elbanton, Kerkdriel, Netherlands) at $27^{\circ} \mathrm{C}$.

\section{Identification and sequence analysis of Pseudocercospora fijiensis genes}

The C. fulvum Avr4 homologue Pfavr4 gene was previously identified by Stergiopoulos et al. (2010, 2014). The ku70 orthologues from $N$. crassa (Ninomiya et al., 2004), R. toruloides (Koh et al., 2014) and the Dothideomycetes Z. tritici (Bowler et al., 2010), P. nodorum (Feng et al., 2012), A. alternata (Wang et al., 2011) and S. musiva (http://genome.jgi.doe.gov/Sepmu1/Sepmu1.home.html) were used as query with the BLASTN search engine at the $P$. fijiensis genome website (http://genome.jgipsf.org/Mycfi2/Mycfi2.home.html). Protein structure was inferred with the NCBI Conserved domains analysis tool. The Pfcyp51 was detected in the P. fijiensis genome strain and amplified in other strains (Diaz-Trujillo et al., 2018).

\section{Constructs for insertion and gene deletion}

Vectors pSC001 or pSC002 were applied for random insertion (Mirzadi Gohari et al., 2015; Zhao et al., 2011). They contain the marker genes eGFP or DsRed, respectively, under control of the pToxA promoter from Pyrenophora tritici-repentis and the terminator Tnos in combination with the hygromycin B phosphotransferase gene $(h p h)$ driven by the PtrpC promoter from A. nidulans. These plasmids were transformed into A. tumefaciens strain LBA1100 by electroporation, as described (Zwiers and De Waard (2001). 
Deletion constructs were generated with three DNA cassettes targeted for the knockout of a gene that was amplified using specific primers, including the attB1 and attB2 sites for Gateway® cloning (Invitrogen). These fragments were then cloned into the $\mathrm{pDONR}^{\mathrm{TM}} 221$ vector, using the Multisite Gateway ${ }^{\circledR}$ Three-fragment Vector Construction kit (Invitrogen, California, USA; Cat No. 12537-023, version D). The cassette with $h p h$ was constructed based on the pSC001 plasmid (Figure 1).

Upstream and downstream fragments for each gene (Figure 1) were identified and amplified using $50 \mathrm{ng}$ genomic DNA from $P$. fijiensis CIRAD86, with $1 \mu \mathrm{M}$ of corresponding primers, $0.2 \mathrm{mM}$ dNTPs, 1x Pfu DNA polymerase 10X with MgSO4 buffer (Promega, Madison, USA), 0.4 units Pfu DNA Polymerase (Promega), 4.1 units TaqDNA polymerase (Roche, Penzberg, Germany), following this program: 5 minutes at $95{ }^{\circ} \mathrm{C}$, followed by 30 cycles of 35 seconds at $95{ }^{\circ} \mathrm{C}$, annealing temperature for 1 minute, then 1 minute and 50 seconds at $72{ }^{\circ} \mathrm{C}$, followed by a final elongation at $72{ }^{\circ} \mathrm{C}$ for 5 minutes. Annealing temperatures were: 72 and $64{ }^{\circ} \mathrm{C}$ for upstream and downstream fragments of Pfavr4 respectively; likewise 72 and $66{ }^{\circ} \mathrm{C}$ for upstream and downstream fragments of $P f k u 70$.

Amplicons were purified by either QIAquick gel extraction or QIAquick PCR purification kits (Qiagen, Venlo, Netherlands) according to the manufacturer's conditions. Upstream and downstream purified fragments were BP-cloned into vectors plasmid $\mathrm{pDONR}^{\mathrm{TM}} \mathrm{P} 4-\mathrm{P} 1 \mathrm{R}$, or $\mathrm{pDONR}{ }^{\mathrm{TM}} \mathrm{P} 2 \mathrm{R}-\mathrm{P} 3$, respectively, while the replacing cassette was $\mathrm{BP}$ cloned into $\mathrm{pDONR}^{\mathrm{TM}} 221$, from the Multisite Gateway® Three-fragment Vector Construction kit according to the manufacturer's suggestions and transformed into E. coli One Shot(R) TOP10 competent cells (Invitrogen, Carlsbad, USA). Plasmids holding the desired construct were used for the subsequent LR reaction for $15 \mathrm{hrs}$, according to the 
manufacturer's recommendations, using the plasmid pPm43GW (VIB, Ghent, Belgium) as destination vector and transformed into E coli.

Two $\mu$ of a pPm43GW plasmid with the desired construct were electroporated (BioRad) into $50 \mu \mathrm{l}$ of $A$. tumefaciens strain AGL1 competent cells at $12.5 \mathrm{kV} / \mathrm{cm}, 25 \mu \mathrm{F}, 400$ Ohm, 9 msecond in a $1 \mathrm{~mm}$ cuvette (CE-001-50, Eurogentec, Seraing, Belgium). Surviving cells were recovered for 4 hours in $500 \mu \mathrm{L} \mathrm{LB}$ mannitol $\left(10 \mathrm{mg} \cdot \mathrm{mL}^{-1}\right)$ at $28{ }^{\circ} \mathrm{C}$, centrifuged at $150 \mathrm{rpm}$ and then plated on LB medium amended with mannitol $\left(10 \mu \mathrm{g} \cdot \mathrm{mL}^{-1}\right)(\mathrm{Cat} \mathrm{M} 1902$

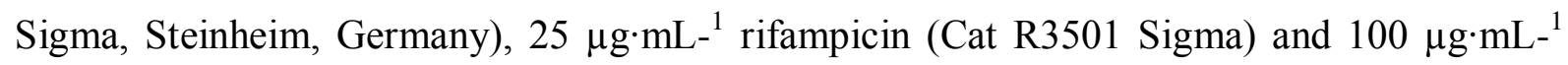
spectinomycin (Cat S4014 Sigma).

\section{Preparation of fungal hyphae for transformation}

A mycelial $P$. fijiensis colony on a PDA plate with a diameter of approximately $1 \mathrm{~cm}$ or $\sim 1$ month old, was transferred into $1.5 \mathrm{~mL}$ of sterile water, amended with $0.05 \%$ Tween20 in a $10 \mathrm{~mL}$ plastic tube (Cat 164161, Greiner Bio-One, Alphen aan de Rijn, Netherlands) and strongly smashed with the straight tip of a plastic Lazy-L-shaped spreader, before vortexing for 40 seconds at the highest speed. This suspension with disaggregated mycelial parts was adjusted up to seven $\mathrm{ml}$ using the same water/Tween 20 mixture. Approximately $0.5 \mathrm{ml}$ of this suspension was transferred onto PDA plates amended with streptomycin $\left(100 \mu \mathrm{g} \cdot \mathrm{mL}^{-1}\right)$. After two to three weeks and one day before transformation, hyphae were rescued from these plates using a brush with plastic firm hairs, in the presence of Induction Medium (IM) (Per liter: $2 \mathrm{~g}$ $\mathrm{K}_{2} \mathrm{HPO}_{4}, 1.45 \mathrm{~g} \mathrm{KH}_{2} \mathrm{PO}_{4}, 0.6 \mathrm{~g} \mathrm{MgSO}_{4} 7 \mathrm{H}_{2} \mathrm{O}, 0.3 \mathrm{~g} \mathrm{NaCl}, 0.01 \mathrm{~g} \mathrm{CaCl}_{2} 2 \mathrm{H}_{2} \mathrm{O}, 0.001 \mathrm{~g} \mathrm{FeSO}_{4}$, $0.5 \mathrm{mg} \mathrm{ZnSO}_{4} 7 \mathrm{H}_{2} \mathrm{O}, 0.5 \mathrm{mg} \mathrm{CuSO}_{4} 5 \mathrm{H}_{2} \mathrm{O}, 0.5 \mathrm{mg} \mathrm{H}_{3} \mathrm{BO}_{3}, 0.5 \mathrm{mg} \mathrm{MnSO}_{4} \mathrm{H}_{2} \mathrm{O}, 0.5 \mathrm{mg}$ $\mathrm{Na}_{2} \mathrm{MoO}_{4} 2 \mathrm{H}_{2} \mathrm{O}, 0.5 \mathrm{~g} \mathrm{NH}_{4} \mathrm{NO}_{3}, 2 \mathrm{~g}$ glucose, $0.2 \mathrm{~mL} 50 \%$ glycerol and $80 \mu \mathrm{l}$ IM MES pH 5.3). Hyphal fragments were then filtered through two layers of sterile gauze swabs (Cat. 708505, Noba, Wetter, Germany) and adjusted to $1.10^{6}$ fragments $\cdot \mathrm{mL}^{-1}$. These were washed 
thrice with IM and taken up in $10 \mathrm{ml}$ IM in a $50 \mathrm{ml}$ tube (Cat 210261, Greiner Bio-One), before an overnight incubation in a rotary shaker (Innova 4430, New Brunswick Scientific, The Netherlands) adjusted at $27^{\circ} \mathrm{C}$ and $150 \mathrm{rpm}$.

\section{Preparation of Agrobacterium cells and Pseudocercospora fijiensis transformation}

Five days before transformation of P. fijiensis, A. tumefaciens LBA1100 cells with the constructs for random mutagenesis were plated in LB medium amended with mannitol (10 $\left.\mathrm{mg} \cdot \mathrm{mL}^{-1}\right), 10 \mu \mathrm{g} \cdot \mathrm{mL}^{-1}$ gentamycin and $250 \mu \mathrm{g} \cdot \mathrm{mL}^{-1}$ spectinomycin. After growing for two days, single colonies were inoculated into $20 \mathrm{~mL}$ LB liquid medium with same antibiotics and incubated overnight in an orbital shaker adjusted at $27{ }^{\circ} \mathrm{C}$ and $150 \mathrm{rpm}$. Cells were resuspended into $20 \mathrm{ml}$ of minimal medium (MM) (same as IM, but without glycerol and MES), and $100 \mu \mathrm{l}$ of this suspension were transferred into $20 \mathrm{ml}$ of fresh $\mathrm{MM}$ with $10 \mu \mathrm{g} \cdot \mathrm{mL}-$ ${ }^{1}$ gentamycin and $250 \mu \mathrm{g} \cdot \mathrm{mL}^{-1}$ spectinomycin and acetosyringone $(0.1 \%$ of $200 \mathrm{mM})$ in a 50 $\mathrm{ml}$ tube. After approximately $24 \mathrm{hrs}$, the $\mathrm{OD}_{600}$ was adjusted to 0.15 with freshly prepared IM (with $10 \mu \mathrm{g} \cdot \mathrm{mL}^{-1}$ gentamycin and $250 \mu \mathrm{g} \cdot \mathrm{mL}^{-1}$ spectinomycin), and incubated for three hrs until an $\mathrm{OD}_{600}$ of 0.25 . Subsequently, hyphal fragments $\left(1.10^{6} \cdot \mathrm{mL}^{-1}\right)$ and A. tumefaciens were mixed in a 1:1 ratio. Aliquots of $1.5 \mathrm{~mL}$ were spread on a $100 \mathrm{~cm}^{2}$ nylon membrane (Amersham Hybond $^{\mathrm{TM}}-\mathrm{N}$, GE Healthcare, Buckinghamshire, UK) layer on IM with $1.5 \%$ agar amended with $10 \mu \mathrm{g} \cdot \mathrm{mL}^{-1}$ gentamycin and $250 \mu \mathrm{g} \cdot \mathrm{mL}^{-1}$ spectinomycin and acetosyringone $(0.1 \%$ of $200 \mathrm{mM})$ (Cat 134406, Sigma-Aldrich) and incubated in the dark for $48 \mathrm{hrs}$ at $22{ }^{\circ} \mathrm{C}$. Membranes were then transferred onto PDA amended with cefotaxime (200 $\mathrm{mM})$ (Cat C7039, Sigma) and hygromycin B $\left(50 \mathrm{mg} \cdot \mathrm{mL}^{-1}\right)$ (Cat H0654, Sigma).

For transformation of $P$. fijiensis with the replacement cassette cloned into $A$. tumefaciens strain AGL1 essentially the same protocol was followed with some modifications. A single A. tumefaciens colony was transferred to a fresh LB agar plate 
containing rifampicin $\left(100 \mu \mathrm{g} \cdot \mathrm{mL}^{-1}\right)$, spectinomycin $\left(100 \mu \mathrm{g} \cdot \mathrm{mL}^{-1}\right)$ and mannitol $\left(10 \mathrm{mg} \cdot \mathrm{mL}^{-}\right.$ ${ }^{1}$ ) and allowed to grow for 36-40 hrs in $20 \mathrm{ml} \mathrm{LB}$ with the same antibiotics in an orbital shaker at $27{ }^{\circ} \mathrm{C}$ and $150 \mathrm{rpm}$. AGL1 cells were resuspended into $20 \mathrm{ml}$ of MM. Immediately $120 \mu \mathrm{l}$ from this suspension were then transferred in $20 \mathrm{ml}$ fresh $\mathrm{MM}$ with antibiotics and incubated again at $27^{\circ} \mathrm{C}$ and $150 \mathrm{rpm}$ in an orbital shaker for approximately $40 \mathrm{hrs}$. Hyphae were rescued from 12 to 15-day-old fungal culture plates in the presence of IM amended with $0.1 \%$ of $200 \mathrm{mM}$ acetosyringone. After filtering hyphae through two layers of gauze swabs (Noba), concentrations were adjusted to $10^{6}$ fragments $\cdot \mathrm{mL}^{-1}$ and washed with IM once by centrifugation at 2,000 rpm for 12 minutes, and incubated overnight in a rotatory shaker at 27 ${ }^{\circ} \mathrm{C}$ and $150 \mathrm{rpm}$ before transformation. During co-cultivation of hyphae with AGL1 cells in a 1:1 ratio on a $100 \mathrm{~cm}^{2}$ nylon membrane (Amersham Hybond ${ }^{\mathrm{TM}}-\mathrm{N}$ ) layer on IM with $1.5 \%$ agar amended with rifampicin $\left(100 \mu \mathrm{g} \cdot \mathrm{mL}^{-1}\right)$, spectinomycin $\left(100 \mu \mathrm{g} \cdot \mathrm{mL}^{-1}\right)$ and acetosyringone $(0.1 \%$ of $200 \mathrm{mM})$ for $48 \mathrm{hrs}$, the temperature was maintained at $27^{\circ} \mathrm{C}$, and selection medium was amended with cefotaxime $(200 \mathrm{mM})$ and hygromycin $\mathrm{B}\left(50 \mu \mathrm{g} \cdot \mathrm{mL}^{-1}\right)$.

\section{Screening of fluorescent transformants}

After 15 days incubation, developing colonies on the membranes were monitored for fluorescence under a fluorescence stereoscope microscope (Leica, Wetzlar, Germany). GFP or DsRed fluorescence was detected with 488 or $563 \mathrm{~nm}$ excitation, and 509 or $581 \mathrm{~nm}$ emission settings, respectively. Images were captured with an Axiocam MRc5 camera using Axioplan 2.0 software (Zeiss, Göttingen, Germany).

\section{Confirmation of gene replacement}

Fluorescent mycelial colonies were rescued with a sterile tooth pick or pipet tip and transferred to a fresh PDA plate amended with $300 \mu \mathrm{g} \cdot \mathrm{mL}^{-1}$ cefatoxime and $50 \mu \mathrm{g} \cdot \mathrm{mL}^{-1}$ hygromycin. After approximately three weeks, mycelium was collected and lyophilized for 
DNA isolation using the Sbeadex (R) Maxi Plant kit (Agowa, Germany) in a KingFisher Robot (Thermo Scientific, Hudson, USA). DNA of transformants was used to amplify the replaced Pfavr4 or Pfku70 with the primers Avr4_F1 + Avr4_R1, or KU70_F1 + KU70_R1, respectively (Table 3). The amplification mixture comprised 20 ng genomic DNA, with 0.75 $\mu \mathrm{M}$ of corresponding primers, $60 \mu \mathrm{M}$ dNTPs (Roche), 1x DNA polymerase with $\mathrm{MgSO}_{4}$ buffer and 0.8 units Taq DNA polymerase (Roche). Pfavr4 was amplified in a thermocycler (PTC200 Peltier, BioRad, Watertown, USA) programmed at 5 min at $95{ }^{\circ} \mathrm{C}, 30$ cycles of 30 sec. at $94{ }^{\circ} \mathrm{C}, 30 \mathrm{sec}$. at $58{ }^{\circ} \mathrm{C}$ and $60 \mathrm{sec}$. at $72{ }^{\circ} \mathrm{C}$, followed by a final elongation for $5 \mathrm{~min}$. at $72{ }^{\circ} \mathrm{C}$. The amplification program for $P f k u 70$ was 5 min. at $95{ }^{\circ} \mathrm{C}$ followed by 30 cycles of $30 \mathrm{sec}$. at $95{ }^{\circ} \mathrm{C}, 45 \mathrm{sec}$. at $58{ }^{\circ} \mathrm{C}$ and $60 \mathrm{sec}$. at $72{ }^{\circ} \mathrm{C}$ and a final elongation for $2 \mathrm{~min}$. at 72 ${ }^{\circ} \mathrm{C}$. The expected amplicons sizes were $364 \mathrm{bp}$ (Pfavr4) and 1,261 bp (Pfku70), respectively. Transformants that lacked a PCR product, but whose DNA amplified with the control variable number tandem repeat VNTR1333 marker according to García et al. (2010), were subjected to an additional PCR with a primer located in the replacing construct (Avr4_HR_F or KU70_HR_F, respectively) and one primer in the UTR region, based on the P. fijiensis CIRAD86 genome sequence (Avr4_HR_R or KU70_HR_R, respectively) (Table 3, Figure 1), using an amplification program of $5 \mathrm{~min}$. at $95^{\circ} \mathrm{C}$ followed by 30 cycles of $30 \mathrm{sec}$. at $95^{\circ} \mathrm{C}$, 60 sec. at $62{ }^{\circ} \mathrm{C}, 180 \mathrm{sec}$. at $72{ }^{\circ} \mathrm{C}$ and a final elongation for eight min. at $72{ }^{\circ} \mathrm{C}$. The expected amplicon sizes for knock-out genes were 2,849 bp for $\Delta$ Pfavr4 mutants or of 2,953 bp for $\triangle P f k u 70$ mutants.

\section{Transformation efficiency and homologous recombination}

Efficiencies of random and directed mutagenesis were calculated as the percentage of transformed colonies among the number of hyphal pieces used for transformation. The 
percentage of transformants with proven homologous recombination by PCR, among the number of transformed colonies, was defined as the frequency of homologous recombination.

\section{Phenotypic characterization of knock-out Pseudocercospora fijiensis strains}

In vitro growth of $\mathrm{Ku} 70$ mutants. The fitness of $P f k u 70$ knockout strains was analyzed by generating hyphal fragments from one-month-old colonies of $\triangle P f k u 70 \# 33$ and $\triangle P f k u 70 \# 45$ and the $P$. fijiensis CIRAD86 wt. For each sample $50 \mu \mathrm{l}$ of $3.5 \cdot 10^{5}$ mycelial fragments $\cdot \mathrm{mL}^{-1}$ were inoculated in $200 \mu \mathrm{l}$ potato dextrose broth medium in eight wells of a 96-well, flat bottom, transparent, polystyrol plate (Cat 3370, Corning, USA) for a final concentration of $7.10^{4}$ mycelial fragments $\cdot \mathrm{mL}^{-1}$ in a total of $250 \mu \mathrm{l}$ medium. Each sample used a column of eight wells and a column with only medium was left adjacent to it. Plates were sealed and maintained in an incubator (Elbanton, Kerkdriel, Netherlands) at $27{ }^{\circ} \mathrm{C}$ in darkness. Mycelial growth was monitored at $0,4,6,8,10$, and 12 days post inoculation (dpi) in two biological and two technical replicates, using an Infinite ${ }^{\circledR}$ M200 PRO machine (TEKAN, Männedorf, Switzerland). The reads from the control wells with only growth medium were subtracted from those with fungal inoculum and fungal growth was measured by increased absorbance values that were averaged, plotted against dpis and compared with the wt strain. Pictures of wells were recorded with an Olympus Camedia C-8080 (Olympus, Hamburg, Germany). 
Acknowledgements

We would like to thank Jean Carlier from the Centre de Coopération Internationale en Recherche Agronomique pour le Développement (CIRAD, Montpellier, France) for the gift of Pseudocercospora fijiensis strains CIRAD86 and CIRAD 139a. Lute-Harm Zwiers (Avans Hogeschool) and Ioannis Stergiopoulos (UC Davis) are acknowledged for fruitful discussions. CDT was supported by The National Council of Science and Technology (CONACyT) from Mexico for $\mathrm{PhD}$ scholarship \# 187781. AHK was supported by the Federal Agency for Support and Evaluation of Graduate Education of Brazil (CAPES) during Embrapa-Labex in Wageningen, The Netherlands. RA is thankful to the Universidad Nacional de Colombia, sede Medellín for funding collaborative research with Wageningen University and Research and Wageningen Plant Research. GHJK and HGJM are thankful for the grant of the Dutch Dioraphte Foundation. 


\section{References}

Abello, J., Kelemu, S., García, C., 2008. Agrobacterium-mediated transformation of the endophytic fungus Acremonium implicatum associated with Brachiaria grasses. Mycol. Res. 112, 407413. doi:10.1016/j.mycres.2007.10.008

Amil, A.F., Heaney, S.P., Stanger, C., Shaw, M.W., 2007. Dynamics of QoI sensitivity in Mycosphaerella fijiensis in Costa Rica during 2000 to 2003. Phytopathology 97, 1451-1457. doi:10.1094/PHYTO-97-11-1451

Arango Isaza, R.E., Díaz-Trujillo, C., Dhillon, B., Aerts, A., Carlier, J., Crane, C.F., Jong, T.V. de, Vries, I. de, Dietrich, R., Farmer, A.D., Fortes Fereira, C., Garcia, S., Guzmán, M., Hamelin, R.C., Lindquist, E.A., Mehrabi, R., Quiros, O., Schmutz, J., Shapiro, H., Reynolds, E., Scalliet, G., Souza Jr, M., Stergiopoulos, I., Van der Lee, T.A.J., Wit, P.J.G.M. de, Zapater, M.-F., Zwiers, L.-H., Grigoriev, I.V., Goodwin, S.B., Kema, G.H.J., 2016. Combating a global threat to a clonal crop: the banana black Sigatoka pathogen Pseudocercospora fijiensis (synonym Mycosphaerella fijiensis) genomes reveals clues for disease control. PLoS Genet 12(8): e1005876. doi:10.1371/journal.pgen.1005876

Balint-Kurti, P.J., May, G.D., Churchill, A.C.L., 2001. Development of a transformation system for Mycosphaerella pathogens of banana: a tool for the study of host/pathogen interactions. FEMS Microbiology Letters 195, 9-15. doi:10.1111/j.1574-6968.2001.tb10490.x

Beltrán-García, M.J., Prado, F.M., Oliveira, M.S., Ortiz-Mendoza, D., Scalfo, A.C., Pessoa, A., Jr, Medeiros, M.H.G., White, J.F., Di Mascio, P., 2014. Singlet molecular oxygen generation by light-activated DHN-melanin of the fungal pathogen Mycosphaerella fijiensis in black Sigatoka disease of bananas. PLoS ONE 9, e91616. doi:10.1371/journal.pone.0091616

Bowler, J., Scott, E., Tailor, R., Scalliet, G., Ray, J., Csukai, M., 2010. New capabilities for Mycosphaerella graminicola research. Mol. Plant Pathol. 11, 691-704. doi:10.1111/j.13643703.2010.00629.x

Burt, P.J.A., 1994. Windborne dispersal of sigatoka leaf spot pathogens. Grana 33, 108-111. doi:10.1080/00173139409427842

Cañas-Gutiérrez, G.P., Patiño, L.F., Rodríguez-Arango, E., Arango, R., 2006. Molecular characterization of benomyl-resistant isolates of Mycosphaerella fijiensis, collected in Colombia. Journal of Phytopathology 154, 403-409. doi:10.1111/j.1439-0434.2006.01113.x

Carlier, J., Lebrun, M.H., Zapater, M.F., Dubois, C., Mourichon, X., 1996. Genetic structure of the global population of banana black leaf streak fungus, Mycosphaerella fijiensis. Molecular Ecology 5, 499-510. doi:10.1111/j.1365-294X.1996.tb00342.x

Chang, P.-K., 2008. A highly efficient gene-targeting system for Aspergillus parasiticus. Lett. Appl. Microbiol. 46, 587-592. doi:10.1111/j.1472-765X.2008.02345.x

Chang, T.-C., Salvucci, A., Crous, P.W., Stergiopoulos, I., 2016. Comparative genomics of the Sigatoka disease complex on banana suggests a link between parallel evolutionary changes in Pseudocercospora fijiensis and Pseudocercospora eumusae and increased virulence on the banana host. PLoS Genetics In press.

Chen, X., Stone, M., Schlagnhaufer, C., Romaine, C.P., 2000. A Fruiting Body Tissue Method for Efficient Agrobacterium-Mediated Transformation of Agaricus bisporus. Appl Environ Microbiol 66, 4510-4513.

Chico, L., Ciudad, T., Hsu, M., Lue, N.F., Larriba, G., 2011. The Candida albicans Ku70 Modulates Telomere Length and Structure by Regulating Both Telomerase and Recombination. PLOS ONE 6, e23732. doi:10.1371/journal.pone.0023732

Chong, P., 2016. Origin, versatility and distribution of azole fungicide resistance in the banana black Sigatoka pathogen Pseudocercospora fijiensis. Wageningen University, Wageningen, the Netherlands.

Choquer, M., Robin, G., Pêcheur, P.L., Giraud, C., Levis, C., Viaud, M., 2008. Ku70 or Ku80 deficiencies in the fungus Botrytis cinerea facilitate targeting of genes that are hard to knock 
out in a wild-type context. FEMS Microbiology Letters 289, 225-232. doi:10.1111/j.15746968.2008.01388.x

Chu, V.T., Weber, T., Wefers, B., Wurst, W., Sander, S., Rajewsky, K., Kühn, R., 2015. Increasing the efficiency of homology-directed repair for CRISPR-Cas9-induced precise gene editing in mammalian cells. Nat. Biotechnol. 33, 543-548. doi:10.1038/nbt.3198

Churchill, A.C.L., 2011. Mycosphaerella fijiensis, the black leaf streak pathogen of banana: progress towards understanding pathogen biology and detection, disease development, and the challenges of control. Molecular Plant Pathology 12, 307-328. doi:10.1111/j.13643703.2010.00672.x

Combier, J.-P., Melayah, D., Raffier, C., Gay, G., Marmeisse, R., 2003. Agrobacterium tumefaciens-mediated transformation as a tool for insertional mutagenesis in the symbiotic ectomycorrhizal fungus Hebeloma cylindrosporum. FEMS Microbiol. Lett. 220, 141-148.

Couoh-Uicab, Y., Islas-Flores, L., Kantún-Moreno, N., Zwiers, L.H., Tzec-Simá, M., Echeverría, S.P.-, Brito-Argáez, L., Peraza-Echeverría, L., Grijalva-Arango, R., James, A., Rodríguez-García, C., Canto-Canché, B., 2013. Cloning, in silico structural characterization and expression analysis of MfAtr4, an ABC transporter from the banana pathogen Mycosphaerella fijiensis. African Journal of Biotechnology 11, 54-79. doi:10.4314/ajb.v11i1.

Cousin, A., Mehrabi, R., Guilleroux, M., Dufresne, M., Van Der Lee, T., Waalwijk, C., Langin, T., Kema, G.H.J., 2006. The MAP kinase-encoding gene MgFus3 of the non-appressorium phytopathogen Mycosphaerella graminicola is required for penetration and in vitro pycnidia formation. Molecular Plant Pathology 7, 269-278. doi:10.1111/j.1364-3703.2006.00337.x

Cruz-Martín, M., Alvarado-Copó, Y., Acosta-Suárez, M., Leiva-Mora, M., Sánchez-García, C., Roque, B., 2011. Effect of the "in vitro"subculture in conidial production and aggressiveness of Mycosphaerella fijiensis Morelet. Centro Agrícola 38, 11-16.

de Boer, P., Bronkhof, J., Dukiḱ, K., Kerkman, R., Touw, H., van den Berg, M., Offringa, R., 2013. Efficient gene targeting in Penicillium chrysogenum using novel Agrobacteriummediated transformation approaches. Fungal genetics and biology: FG \& B 61, 9-14. doi:10.1016/j.fgb.2013.08.012

Deighton, F.C., 1976. Studies on Cercospora and allied genera. VI. Pseudocercospora Cif. and Cercoseptoria Petr. Mycol. Pap. 140, 1-168

De Lapeyre De Bellaire, L., Abadie, C., Carlier, J., Ngando, J. and Kema, G.H.J. 2010. Mycosphaerella foliar diseases of bananas: towards an integrated protection. From Science to Field Banana Case Study-Guide 2. ENDURE, Paris (FRA). 8p. http://www.endurenetwork.eu/about_endure/all_the_news/new_guides_for_better_banana_production

de Wit, P.J.G.M. 2016. Cladosporium fulvum effectors: weapons in the arms race with tomato. Annu. Rev. Phytopathol. 54:1, 1-23.

de Wit, P.J.G.M., van der Burgt, A., Okmen, B., Stergiopoulos, I., Abd-Elsalam, K.A., Aerts, A.L., Bahkali, A.H., Beenen, H.G., Chettri, P., Cox, M.P., Datema, E., de Vries, R.P., Dhillon, B., Ganley, A.R., Griffiths, S.A., Guo, Y., Hamelin, R.C., Henrissat, B., Kabir, M.S., Jashni, M.K., Kema, G., Klaubauf, S., Lapidus, A., Levasseur, A., Lindquist, E., Mehrabi, R., Ohm, R.A., Owen, T.J., Salamov, A., Schwelm, A., Schijlen, E., Sun, H., van den Burg, H.A., van Ham, R.C.H.J., Zhang, S., Goodwin, S.B., Grigoriev, I.V., Collemare, J., Bradshaw, R.E., 2012. The Genomes of the Fungal Plant Pathogens Cladosporium fulvum and Dothistroma septosporum Reveal Adaptation to Different Hosts and Lifestyles But Also Signatures of Common Ancestry. PLoS Genet 8. doi:10.1371/journal.pgen.1003088

Diaz-Trujillo, C., Chong, P., Stergiopoulos, I., Cordovez, V., Guzman, M., De Wit, P. J.G.M., Meijer, H. J.G., Arango Isaza, R. E., Scalliet, G., Sierotzki, H., Lilia Peralta, E. and Kema, G. H.J. 2018. A new mechanism for reduced sensitivity to demethylation-inhibitor fungicides in the fungal banana black Sigatoka pathogen Pseudocercospora fijiensis. Mol Plant Pathol. In press. doi:10.1111/mpp.12637

Ding, Y., Liang, S., Lei, J., Chen, L., Kothe, E., Ma, A., 2011. Agrobacterium tumefaciens mediated fused egfp-hph gene expression under the control of gpd promoter in Pleurotus ostreatus. Microbiological Research 166, 314-322. doi:10.1016/j.micres.2010.07.001 
Donzelli, B.G.G., Churchill, A.C.L., 2007. A Quantitative Assay Using Mycelial Fragments to Assess Virulence of Mycosphaerella fijiensis. Phytopathology 97, 916-929. doi:10.1094/PHYTO-97-8-0916

Eckert, M., Maguire, K., Urban, M., Foster, S., Fitt, B., Lucas, J., Hammond-Kosack, K., 2005. Agrobacterium tumefaciens-mediated transformation of Leptosphaeria spp. and Oculimacula spp. with the reef coral gene DsRed and the jellyfish gene gfp. FEMS Microbiology Letters 253, 67-74. doi:10.1016/j.femsle.2005.09.041

El-Khoury, R., Sellem, C.H., Coppin, E., Boivin, A., Maas, M.F.P.M., Debuchy, R., SainsardChanet, A., 2008. Gene deletion and allelic replacement in the filamentous fungus Podospora anserina. Curr Genet 53, 249-258. doi:10.1007/s00294-008-0180-3

Escobar-Tovar, L., Magaña-Ortíz, D., Fernández, F., Guzmán-Quesada, M., SandovalFernández, J.A., Ortíz-Vázquez, E., Loske, A.M., Gómez-Lim, M.A., 2015. Efficient transformation of Mycosphaerella fijiensis by underwater shock waves. J. Microbiol. Methods 119, 98-105. doi:10.1016/j.mimet.2015.10.006

Ezra, D., Skovorodnikova, J., Kroitor-Keren, T., Denisov, Y., Liarzi, O., 2010. Development of methods for detection and Agrobacterium-mediated transformation of the sterile, endophytic fungus Muscodor albus. Biocontrol science and technology.20, 83-97.

FAO, 2003. The World Banana Economy, 1985-2002. Food and Agriculture Organization of the United Nations, Rome.

Fell, V.L., Schild-Poulter, C., 2015. The Ku heterodimer: Function in DNA repair and beyond. Mutation Research/Reviews in Mutation Research 763, 15-29. doi:10.1016/j.mrrev.2014.06.002

Feng, J., Li, W., Hwang, S.-F., Gossen, B.D., Strelkov, S.E., 2012. Enhanced gene replacement frequency in KU70 disruption strain of Stagonospora nodorum. Microbiological Research 167, 173-178. doi:10.1016/j.micres.2011.05.004

Fitzgerald, A.M., Mudge, A.M., Gleave, A.P., Plummer, K.M., 2003. Agrobacterium and PEGmediated transformation of the phytopathogen Venturia inaequalis. Mycological Research 107, 803-810. doi:10.1017/S0953756203008086

FOOD AND AGRICULTURE ORGANIZATION OF THE UNITED NATIONS, 2015. FAOSTAT [WWW Document]. URL http://faostat3.fao.org/home/E (accessed 9.28.16).

FRAC, 2010. FRAC Recommendations for fungicide mixtures - January 2010.

Frandsen, R.J.N., 2011. A guide to binary vectors and strategies for targeted genome modification in fungi using Agrobacterium tumefaciens-mediated transformation. J. Microbiol. Methods 87, 247-262. doi:10.1016/j.mimet.2011.09.004

Fullerton, R.A., Olsen, T.L., 1995. Pathogenic variability in Mycosphaerella fijiensis Morelet, cause of black Sigatoka in banana and plantain. N. Z. J. Crop Hortic. Sci. 23, 39-48. doi:10.1080/01140671.1995.9513866

Garcia, S. a. L., Van der Lee, T. a. J., Ferreira, C.F., Te Lintel Hekkert, B., Zapater, M.-F., Goodwin, S.B., Guzmán, M., Kema, G.H.J., Souza, M.T., 2010. Variable number of tandem repeat markers in the genome sequence of Mycosphaerella fijiensis, the causal agent of black leaf streak disease of banana (Musa spp). Genet. Mol. Res. 9, 2207-2212. doi:10.4238/vol9-4gmr934

Hayden, H.L., Carlier, J., 2003. Genetic structure of Mycosphaerella fijiensis populations from Australia, Papua New Guinea and the Pacific Islands. Plant Pathology 52, 703-712. doi:10.1111/j.1365-3059.2003.00883.x

He, Y., Liu, Q., Shao, Y., Chen, F., 2013. Ku70 and ku80 null mutants improve the gene targeting frequency in Monascus ruber M7. Appl. Microbiol. Biotechnol. 97, 4965-4976. doi:10.1007/s00253-013-4851-8

Hoff, B., Kamerewerd, J., Sigl, C., Zadra, I., Kück, U., 2010. Homologous recombination in the antibiotic producer Penicillium chrysogenum: strain $\triangle P c k u 70$ shows up-regulation of genes from the HOG pathway. Appl Microbiol Biotechnol 85, 1081-1094. doi:10.1007/s00253-0092168-4

Kantún-Moreno, N., Vázquez-Euán, R., Tzec-Simá, M., Peraza-Echeverría, L., GrijalvaArango, R., Rodríguez-García, C., James, A.C., Ramírez-Prado, J., Islas-Flores, I., 
Canto-Canché, B., 2013. Genome-wide in silico identification of GPI proteins in Mycosphaerella fijiensis and transcriptional analysis of two GPI-anchored $\beta-1,3-$ glucanosyltransferases. Mycologia 105, 285-296. doi:10.3852/12-103

Kema, G.H.J., Mirzadi-Gohari A., Aouini, L., Gibriel, H.A.Y., Ware, S.B., van den Bosh, F., Manning-Smith, R., Alonso-Chavez, V., Helps, J., Ben M'Barek, S., Mehrabi, R., DiazTrujillo, C., Zamani, E., Schouten, H.J., van der Lee, T.A.J., Waalwijk, C., de Waard. M.A., de Wit, P.J.G.M., Verstappen, EC.P., Thoma, B.P.H.J., Meijer, H.J.G., Seidl, M.F. 2018. Stress and sexual reproduction affect th dynamics of the wheat pathogen effector AvrStb6 and strobilurin resistance. Nature Genet. In press.

Koh, C.M.J., Liu, Y., Moehninsi, Du, M., Ji, L., 2014. Molecular characterization of KU70 and KU80 homologues and exploitation of a KU70-deficient mutant for improving gene deletion frequency in Rhodosporidium toruloides. BMC Microbiology 14, 50. doi:10.1186/1471-2180$14-50$

Krappmann, S., Sasse, C., Braus, G.H., 2006. Gene Targeting in Aspergillus fumigatus by Homologous Recombination Is Facilitated in a Nonhomologous End- Joining-Deficient Genetic Background. Eukaryot Cell 5, 212-215. doi:10.1128/EC.5.1.212-215.2006

Kück, U., Hoff, B., 2010. New tools for the genetic manipulation of filamentous fungi. Appl Microbiol Biotechnol 86, 51-62. doi:10.1007/s00253-009-2416-7

Lepoivre, P., Busogoro, J.P., Etame, J.J., El Hadrami, A., Carlier, J., Harelimana, G., Mourichon, X., Panis, B., Stella Riveros, A., Sallé, G., Strosse, H., Swennen, R., 2003. Introduction - Banana-Mycosphaerella fijiensis interactions, in: Mycosphaerella Leaf Spot Diseases of Bananas: Present Status and Outlook. Proceedings of the Workshop on Mycosphaerella Leaf Spot Diseases Held in San Jose, Costa Rica on 20-23 May 2002. INIBAP, Montpellier, France, pp. 151-160.

Li, H.-M., Virtudazo, E.V., Toh-e, A., Yamaguchi, M., Kawamoto, S., Shimizu, K., 2010. Nonhomologous end joining pathway of the human pathogen Cryptococcus neoformans influences homologous integration efficiency but not virulence. Mycoscience 51, 272-280. doi:10.1007/s10267-010-0038-7

Li, Z.-H., Du, C.-M., Zhong, Y.-H., Wang, T.-H., 2010. Development of a highly efficient gene targeting system allowing rapid genetic manipulations in Penicillium decumbens. Appl Microbiol Biotechnol 87, 1065-1076. doi:10.1007/s00253-010-2566-7

Liu, R., Chen, L., Jiang, Y., Zhou, Z., Zou, G., 2015. Efficient genome editing in filamentous fungus Trichoderma reesei using the CRISPR/Cas9 system. Cell Discovery 1. doi:10.1038/celldisc. 2015.7

Liu, T., Liu, L., Jiang, X., Hou, J., Fu, K., Zhou, F., Chen, J., 2009. Agrobacterium-mediated transformation as a useful tool for the molecular genetic study of the phytopathogen Curvularia lunata. Eur J Plant Pathol 126, 363-371. doi:10.1007/s10658-009-9541-0

Martínez-Bolaños, L., Téliz-Ortiz, D., Rodríguez-Maciel, J.C., Mora-Aguilera, J.A., Nieto-Ángel, D., Cortés-Flores, J.I., Mejía-Sánchez, D., Nava-Diaz, C., Silva-Aguayo, G., 2012. Resistencia a fungicidas en poblaciones de Mycosphaerella fijiensis del sureste mexicano. Agrociencia 46, 707-717.

Michelmore, R. W., Coaker, G., Bart, R., Beattie, G. A., Bent, A., Bruce, T., Cameron, D., Dangl, J., Dinesh-Kumar, S., Edwards, R., Eves-van den Akker, S., Gassmann, W., Greenberg, J. T., Hanley-Bowdoin, L., Harrison, R. J., Harvey, J., He, P., Huffaker, A., Hulbert, S., Innes, R., Jones, J. D. G., Kaloshian, I., Kamoun, S., Katagiri, F., Leach, J., Ma, W., McDowell, J., Medford, J., Meyers, B., Nelson, R., Oliver, R., Qi, Y., Saunders, D., Shaw, M., Smart, C., Subudhi, P., Torrance, L., Tyler, B., Valent, B., and Walsh, J. 2017. Foundational and translational research opportunities to improve plant health. Mol. PlantMicrobe Interact. 30:515-516. doi:10.1094/MPMI-01-17-0010-CR

Michielse, C.B., Hooykaas, P.J.J., Hondel, C.A.M.J.J. van den, Ram, A.F.J., 2005. Agrobacterium-mediated transformation as a tool for functional genomics in fungi. Curr Genet 48, 1-17. doi:10.1007/s00294-005-0578-0

Mirzadi Gohari, A., 2015. Identification and functional characterization of putative (a)virulence factors in the fungal wheat pathogen Zymoseptoria tritici. Wageningen University, Wageningen, the Netherlands. 
Mirzadi Gohari, A., Ware, S.B., Wittenberg, A.H.J., Mehrabi, R., Ben M'Barek, S., Verstappen, E.C.P., van der Lee, T.A.J., Robert, O., Schouten, H.J., de Wit, P.J.G.M., Kema, G.H.J. 2015. Effector discovery in the fungal wheat pathogen Zymoseptoria tritici. Mol. Plant Pathol. 16:1364-3703. doi: 10.1111/mpp.12251.

Mirzadi Gohari, A., Mehrabi, R., Robert, O., Ince, I.A., Boeren, S., Schuster, M., Steinberg, G., de Wit, P.J.G.M., Kema, G.H.J., 2014. Molecular characterization and functional analyses of ZtWorl, a transcriptional regulator of the fungal wheat pathogen Zymoseptoria tritici. Mol. Plant Pathol. 15, 394-405. doi:10.1111/mpp.12102

Mohan, J., Swennen, R. (Eds.), 2004. Banana Improvement: cellular, molecular biology and induced mutations. Presented at the 4th and final FAO/IAEA research coordination meeting, Science Publishers, Leuven, Belgium.

Morelet, M., 1969. Micromycetes du Var et d'ailleurs (2me Note). Annales de la Société des Sciences Naturelles et d'Archeologic de Toulon et du Var 21, 104-106.

Näätsaari, L., Mistlberger, B., Ruth, C., Hajek, T., Hartner, F.S., Glieder, A., 2012. Deletion of the Pichia pastoris KU70 homologue facilitates platform strain generation for gene expression and synthetic biology. PLoS ONE 7, e39720. doi:10.1371/journal.pone.0039720

Natsume, T., Egusa, M., Kodama, M., Johnson, R., Itoh, T., Itoh, Y., 2004. An appropriate increase in the transcription of Aspergillus nidulans uvsC improved gene targeting efficiency. Biosci. Biotechnol. Biochem. 68, 1649-1656. doi:10.1271/bbb.68.1649

Nayak, T., Szewczyk, E., Oakley, C.E., Osmani, A., Ukil, L., Murray, S.L., Hynes, M.J., Osmani, S.A., Oakley, B.R., 2006. A versatile and efficient gene-targeting system for Aspergillus nidulans. Genetics 172, 1557-1566. doi:10.1534/genetics.105.052563

Ninomiya, Y., Suzuki, K., Ishii, C., Inoue, H., 2004. Highly efficient gene replacements in Neurospora strains deficient for nonhomologous end-joining. PNAS 101, 12248-12253. doi:10.1073/pnas.0402780101

Nizam, S., Singh, K., Verma, P.K., 2010. Expression of the fluorescent proteins DsRed and EGFP to visualize early events of colonization of the chickpea blight fungus Ascochyta rabiei. Curr. Genet. 56, 391-399. doi:10.1007/s00294-010-0305-3

Noar, R.D., Daub, M.E., 2016a. Bioinformatics Prediction of Polyketide Synthase Gene Clusters from Mycosphaerella fijiensis. PLoS ONE 11, e0158471. doi:10.1371/journal.pone.0158471

Noar, R.D., Daub, M.E., 2016b. Transcriptome sequencing of Mycosphaerella fijiensis during association with Musa acuminata reveals candidate pathogenicity genes. BMC Genomics 17 , 690. doi:10.1186/s12864-016-3031-5

Nødvig, C.S., Nielsen, J.B., Kogle, M.E., Mortensen, U.H., 2015. A CRISPR-Cas9 System for Genetic Engineering of Filamentous Fungi. PLoS ONE 10, e0133085. doi:10.1371/journal.pone.0133085

Nyilasi, I., Acs, K., Papp, T., Nagy, E., Vágvölgyi, C., 2005. Agrobacterium tumefaciens-mediated transformation of Mucor circinelloides. Folia Microbiol. (Praha) 50, 415-420.

Ohm, R.A., Feau, N., Henrissat, B., Schoch, C.L., Horwitz, B.A., Barry, K.W., Condon, B.J., Copeland, A.C., Dhillon, B., Glaser, F., Hesse, C.N., Kosti, I., LaButti, K., Lindquist, E.A., Lucas, S., Salamov, A.A., Bradshaw, R.E., Ciuffetti, L., Hamelin, R.C., Kema, G.H.J., Lawrence, C., Scott, J.A., Spatafora, J.W., Turgeon, B.G., de Wit, P.J.G.M., Zhong, S., Goodwin, S.B., Grigoriev, I.V., 2012. Diverse Lifestyles and Strategies of Plant Pathogenesis Encoded in the Genomes of Eighteen Dothideomycetes Fungi. PLoS Pathog 8, e1003037. doi:10.1371/journal.ppat.1003037

Okole, B.N., Schulz, F.A., 1997. Selection of Mycosphaerella fijiensis-resistant cell lines from microcross sections of banana and plantain. Plant Cell Reports 16, 339-343. doi:10.1007/BF01088293

Ortiz, R., Swennen, R., 2014. From crossbreeding to biotechnology-facilitated improvement of banana and plantain. Biotechnology Advances, Plant Biotechnology 2013: "Green for Good II". 32, 158-169. doi:10.1016/j.biotechadv.2013.09.010

Onyilo, F., Tusiime, G., Chen, L.-H., Falk, B., Stergiopoulos, I., Tripathi, J. N., Tushemereirwe, W., Kubiriba, J., Changa, C.,Tripathi, L. (2017). Agrobacterium tumefaciens-mediated transformation of Pseudocercospora fijiensis to determine the role of PfHogl in ssmotic Stress 
regulation and virulence modulation. Front Microbiol, $8, \quad 830$. http://doi.org/10.3389/fmicb.2017.00830

Panganiban, L., Cortes-Maramba, N., Dioquino, C., Suplido, M.L., Ho, H., Francisco-Rivera, A., Manglicmot-Yabes, A., 2004. Correlation between blood ethylenethiourea and thyroid gland disorders among banana plantation workers in the Philippines. Environ Health Perspect 112, 42-45.

Penagos, H.G., 2002. Contact dermatitis caused by pesticides among banana plantation workers in Panama. Int J Occup Environ Health 8, 14-18. doi:10.1179/oeh.2002.8.1.14

Peraza-Echeverría, L., Rodríguez-García, C.M., Zapata-Salazar, D.M., 2008. A rapid, effective method for profuse in vitro conidial production of Mycosphaerella fijiensis. Australasian Plant Pathology 37, 460-463. doi:10.1071/AP08042

Portal, O., Acosta-Suárez, M., Ocaña, B., Schäfer, W., Jiménez, E., Höfte, M., 2012. A green fluorescent protein-transformed Mycosphaerella fijiensis strain shows increased aggressiveness on banana. Australasian Plant Pathol. 41, 645-647. doi:10.1007/s13313-0120155-1

Rivas, G.-G., Zapater, M.-F., Abadie, C., Carlier, J., 2004. Founder effects and stochastic dispersal at the continental scale of the fungal pathogen of bananas Mycosphaerella fijiensis. Molecular Ecology 13, 471-482. doi:10.1046/j.1365-294X.2003.02043.X

Schoch, C.L., Shoemaker, R.A., Seifert, K.A., Hambleton, S., Spatafora, J.W., Crous, P.W., 2006. A multigene phylogeny of the Dothideomycetes using four nuclear loci. Mycologia 98 , 1041-1052. doi:10.3852/mycologia.98.6.1041

Sepúlveda, L., Vásquez, L.E., Paniagua, C.I., Echeverry, D., Hernández, C.A., Rodríguez, E., Restrepo, L.F., Arango, R., 2009. The presence and spectrum of light influences the in vitro conidia production of Mycosphaerella fijiensis causal agent of black Sigatoka. Australasian Plant Pathology 38, 514-517. doi:10.1071/AP09036

Shafran, H., Miyara, I., Eshed, R., Prusky, D., Sherman, A., 2008. Development of new tools for studying gene function in fungi based on the Gateway system. Fungal Genet. Biol. 45, 11471154. doi:10.1016/j.fgb.2008.04.011

Stergiopoulos, I., Burg, H.A. van den, Ökmen, B., Beenen, H.G., Liere, S. van, Kema, G.H.J., Wit, P.J.G.M. de, 2010. Tomato Cf resistance proteins mediate recognition of cognate homologous effectors from fungi pathogenic on dicots and monocots. PNAS 107, 7610-7615. doi:10.1073/pnas. 1002910107

Stergiopoulos, I., Cordovez, V., Ökmen, B., Beenen, H.G., Kema, G.H.J., de Wit, P.J.G.M., 2014. Positive selection and intragenic recombination contribute to high allelic diversity in effector genes of Mycosphaerella fijiensis, causal agent of the black leaf streak disease of banana. Molecular Plant Pathology 15, 447-460. doi:10.1111/mpp.12104

van den Bosch, F., Oliver, R., van den Berg, F., Paveley, N. 2014. Governing principles can guide fungicide-resistance management tactics. Annu. Rev. Phytopathol. 52:4266-4286. doi: 10.1146/annurev-phyto-102313-050158

Wang, N.-Y., Yang, S.L., Lin, C.-H., Chung, K.-R., 2011. Gene inactivation in the citrus pathogenic fungus Alternaria alternata defect at the Ku70 locus associated with non-homologous end joining. World J Microbiol Biotechnol 27, 1817-1826. doi:10.1007/s11274-010-0640-z

Weld, R.J., Eady, C.C., Ridgway, H.J., 2006. Agrobacterium-mediated transformation of Sclerotinia sclerotiorum. J. Microbiol. Methods 65, 202-207. doi:10.1016/j.mimet.2005.07.010

Wenderoth, M., Pinecker, C., Voß, B., Fischer, R., 2017. Establishment of CRISPR/Cas9 in Alternaria alternata. Fungal Genetics and Biology 101, 55-60. doi:10.1016/j.fgb.2017.03.001

Weyda, I., Yang, L., Vang, J., Ahring, B.K., Lübeck, M., Lübeck, P.S., 2017. A comparison of Agrobacterium-mediated transformation and protoplast-mediated transformation with CRISPR-Cas9 and bipartite gene targeting substrates, as effective gene targeting tools for Aspergillus carbonarius. Journal of Microbiological Methods 135, 26-34. doi:10.1016/j.mimet.2017.01.015

Wu, B., Macielog, A.I., Hao, W. 2017. Origin and spread of spliceosomal introns: insights from the fungal clade Zymoseptoria. Genome Bol. Evol. 9:2658-2667. doi: 10.1093/gbe/evx211 
Xue, C., Wu, D., Condon, B.J., Bi, Q., Wang, W., Turgeon, B.G., 2013. Efficient Gene Knockout in the Maize Pathogen Setosphaeria turcica Using Agrobacterium tumefaciens-Mediated Transformation. Phytopathology 103, 641-647. doi:10.1094/PHYTO-08-12-0199-R

Zhao, C., Waalwijk, C., de Wit, P.J.G.M., van der Lee, T., Tang, D., 2011. EBR1, a Novel Zn2Cys6 Transcription Factor, Affects Virulence and Apical Dominance of the Hyphal Tip in Fusarium graminearum. MPMI 24, 1407-1418. doi:10.1094/MPMI-06-11-0158

Zheng, Z., Huang, C., Cao, L., Xie, C., Han, R., 2011. Agrobacterium tumefaciens-mediated transformation as a tool for insertional mutagenesis in medicinal fungus Cordyceps militaris. Fungal Biology 115, 265-274. doi:10.1016/j.funbio.2010.12.011

Zwiers, L.H., De Waard, M.A., 2001. Efficient Agrobacterium tumefaciens-mediated gene disruption in the phytopathogen Mycosphaerella graminicola. Curr. Genet. 39, 388-393. 
A
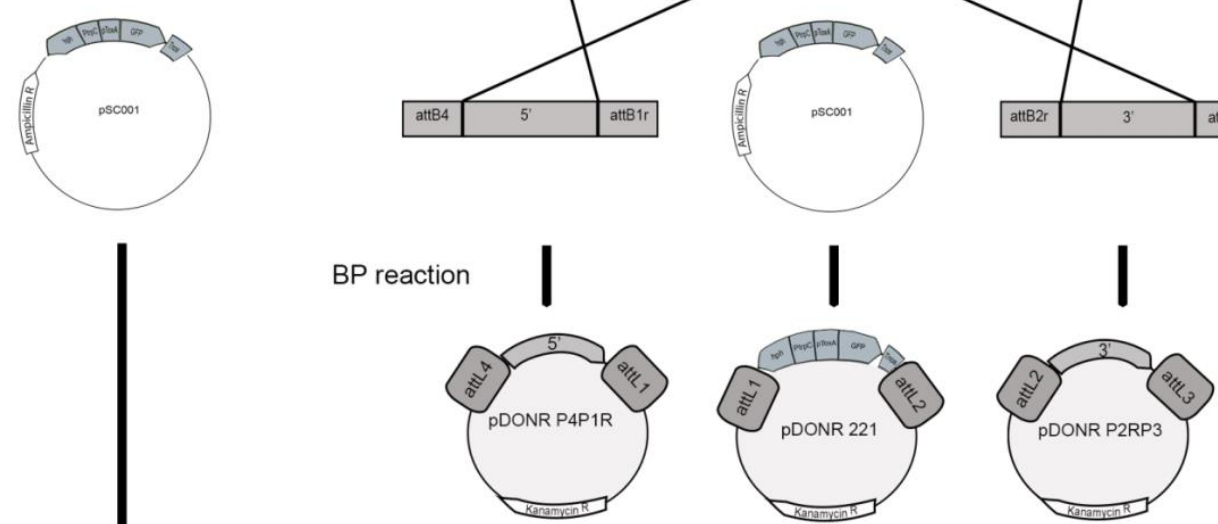

LR reaction
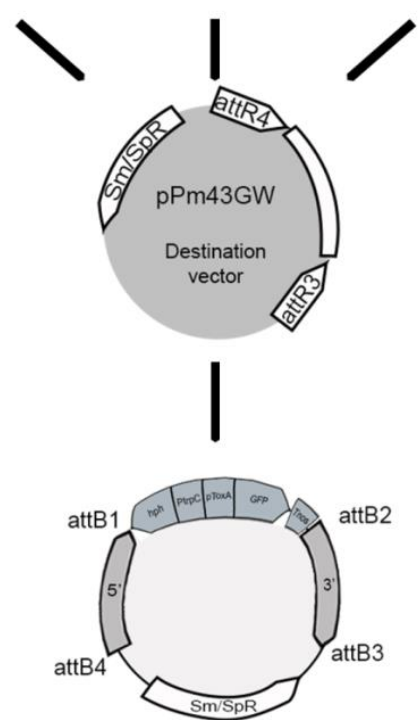

I

AGL1

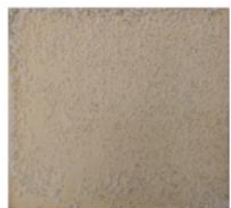

Figure 1. Cloning strategy for Agrobacterium-mediated mutagenesis of Pseudocercospora fijiensis and examples of membranes with the number of obtained transformant colonies. A) Strategy for cloning the construct with eGFP/DsRed and $h p h$ coding genes into the LBA1100 Agrobacterium tumefaciens strain for random mutagenesis. B) Gateway-based strategy for cloning the construct carrying an $e G F P$ and $h p h$ coding genes into the AGL1 $A$. tumefaciens strain to knock out genes Pfavr4 and Pfku70. 
A

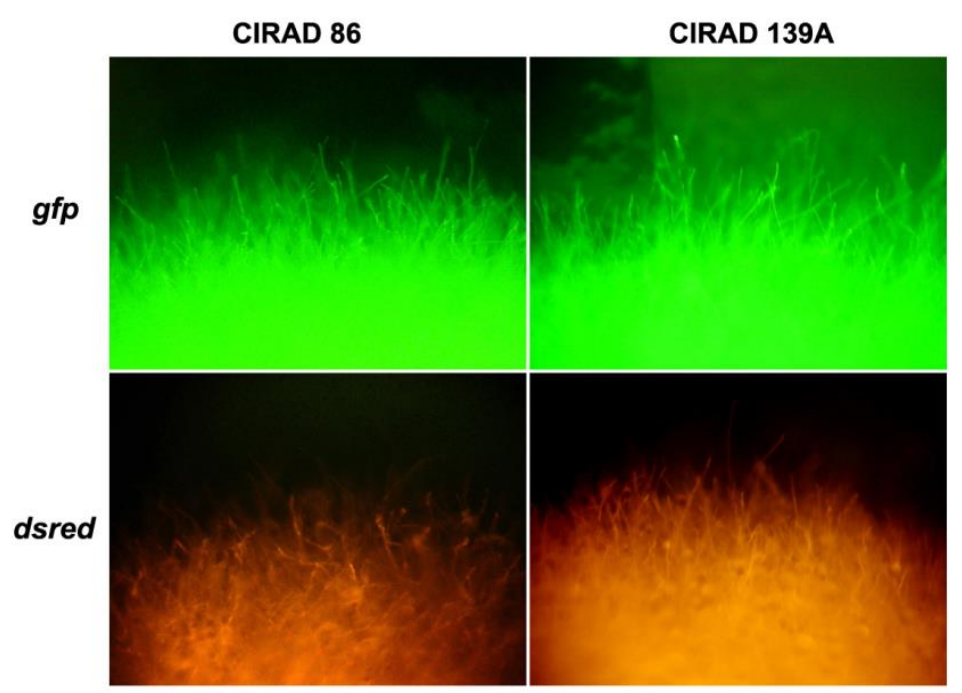

B

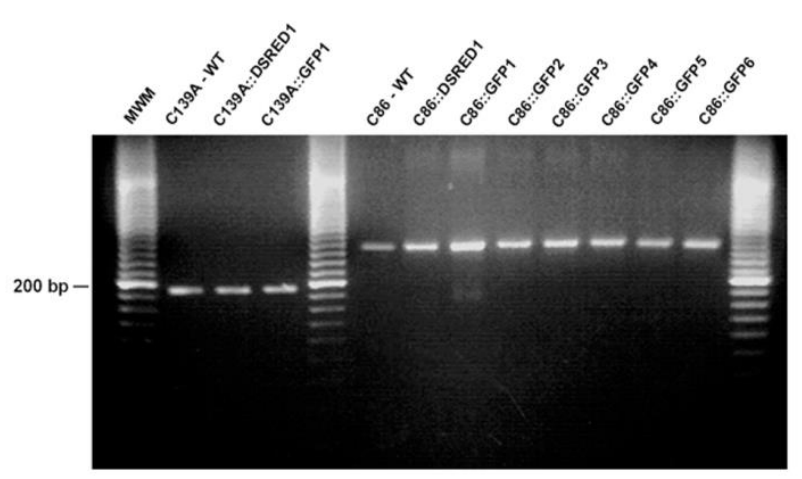

Figure 2 Transformants of Pseudocercospora fijiensis isolates CIRAD86 and CIRAD139A. A) Both P. fijiensis CIRAD86 and CIRAD139A expressing either GFP or DsRed. B) Genotyping transformants using the VNTR1333 marker. CIRAD139A contains the VNTR200 bp allele whereas CIRAD86 contains the 280 bp allele. 
A

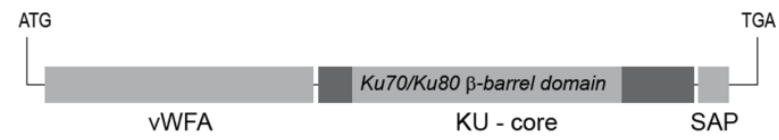

B

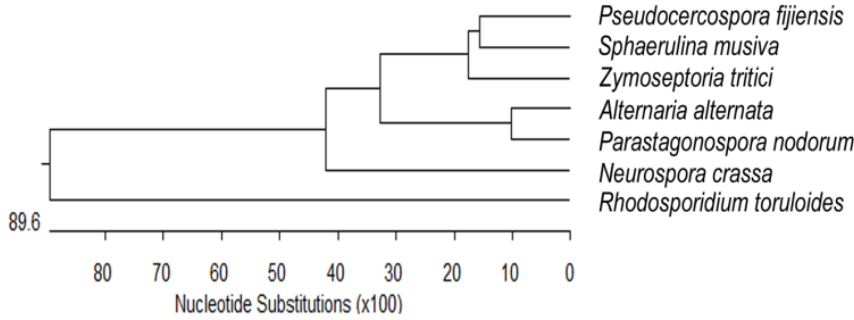

Figure 3. The Pseudocercospora fijiensis KU70 (PfKU70) protein. A) PfKU70 structure. The fused vWA domain and $\mathrm{N}$ terminus span from 36 to 266 aa. The KU-core domain spans 286 to 568 aa and covers the Ku70/Ku80 $\beta$-barrel domain from 293 to 491 aa. The SAP domain is located from 631 till 665 aa. B) Phylogenetic tree of Ku70 proteins of the Dothideomycetes Sphaerulina musiva, Zymoseptoria tritici, Alternaria alternata, Parastagonospora nodorum, Neurospora crassa and the basidiomycete Rhodosporidium toruloides. 

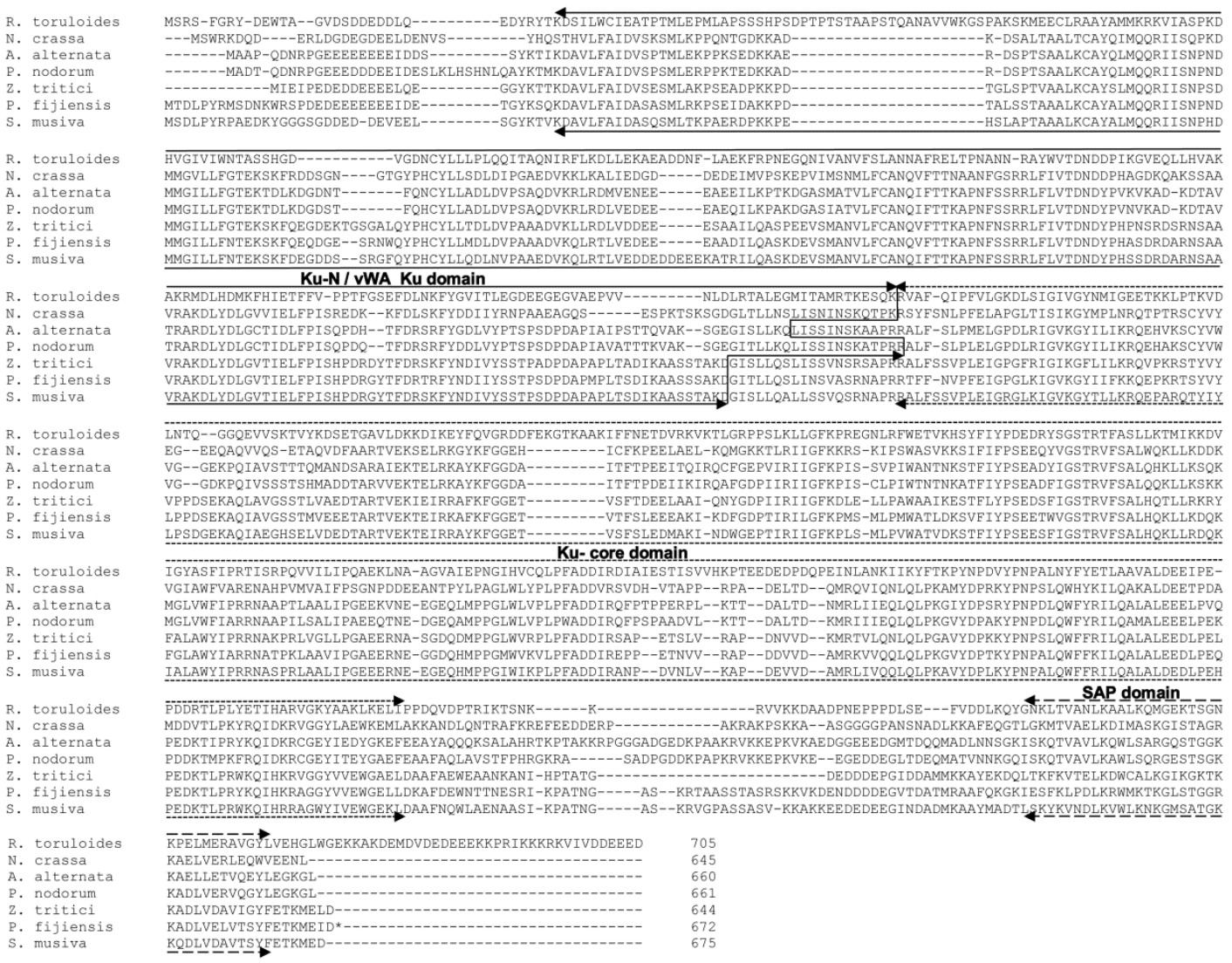

Figure 4. Clustal alignment of KU70 protein from Pseudocercospora fijiensis, Rhodosporium toruloides, Neurospora crassa, Alternaria alternata, Parastagonospora nodorum, Zymoseptoria tritici and Sphaerulina musiva. Conserved domains are labelled with arrows and their respective names. 
A

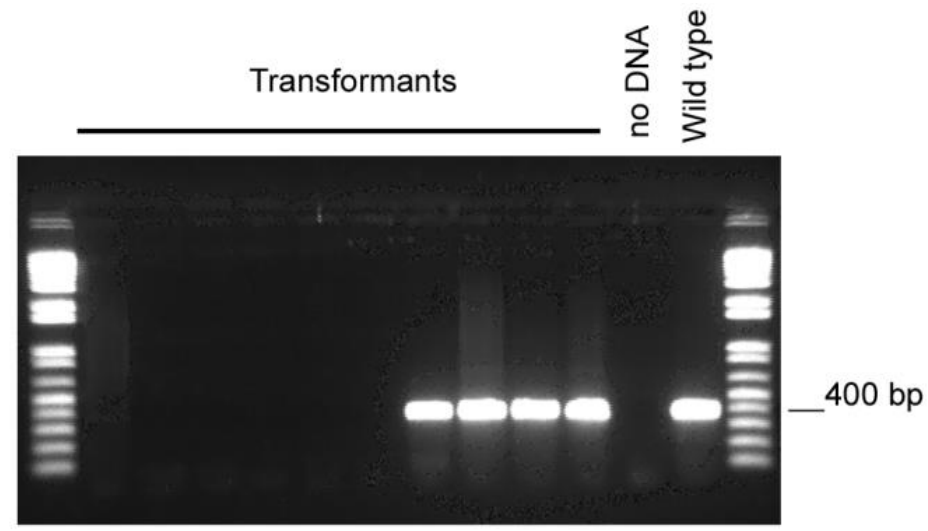

B

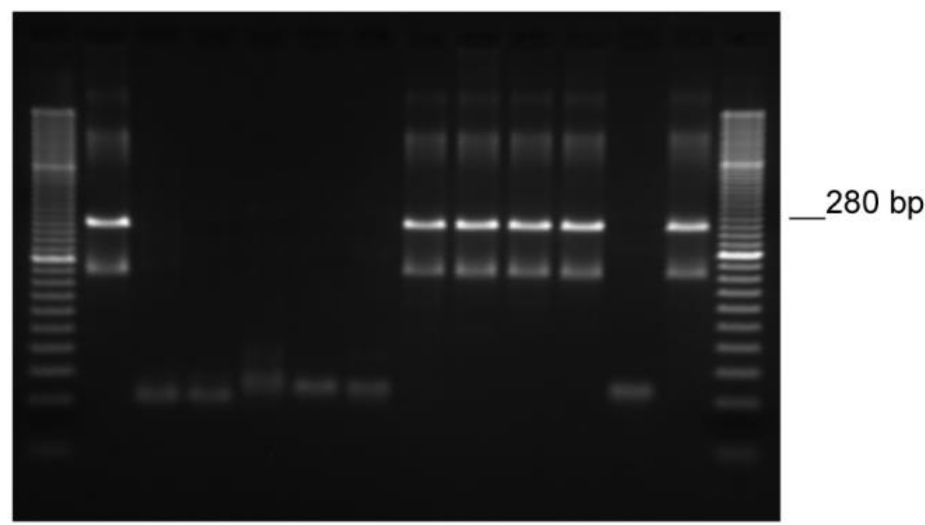

C

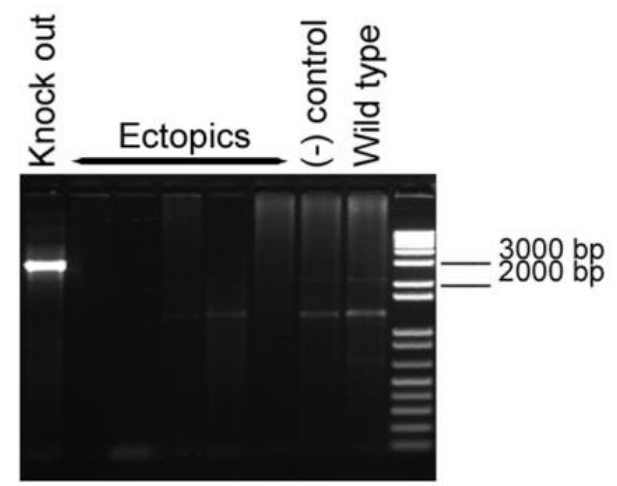

Figure 5. Disruption of Pfavr4 in Pseudocercospora fijiensis CIRAD86. A) Example of transformants with or without amplification of the Pfavr4 region of $364 \mathrm{bp}$, which is not amplified in knock-out strains. B) Only one knock-out candidate amplified the same VNTR1333 allele as the wt CIRAD86 strain, and produced the 2,849 bp amplicons representing the single homologous recombinant PfAvr4 strain $(\mathrm{C})$. 


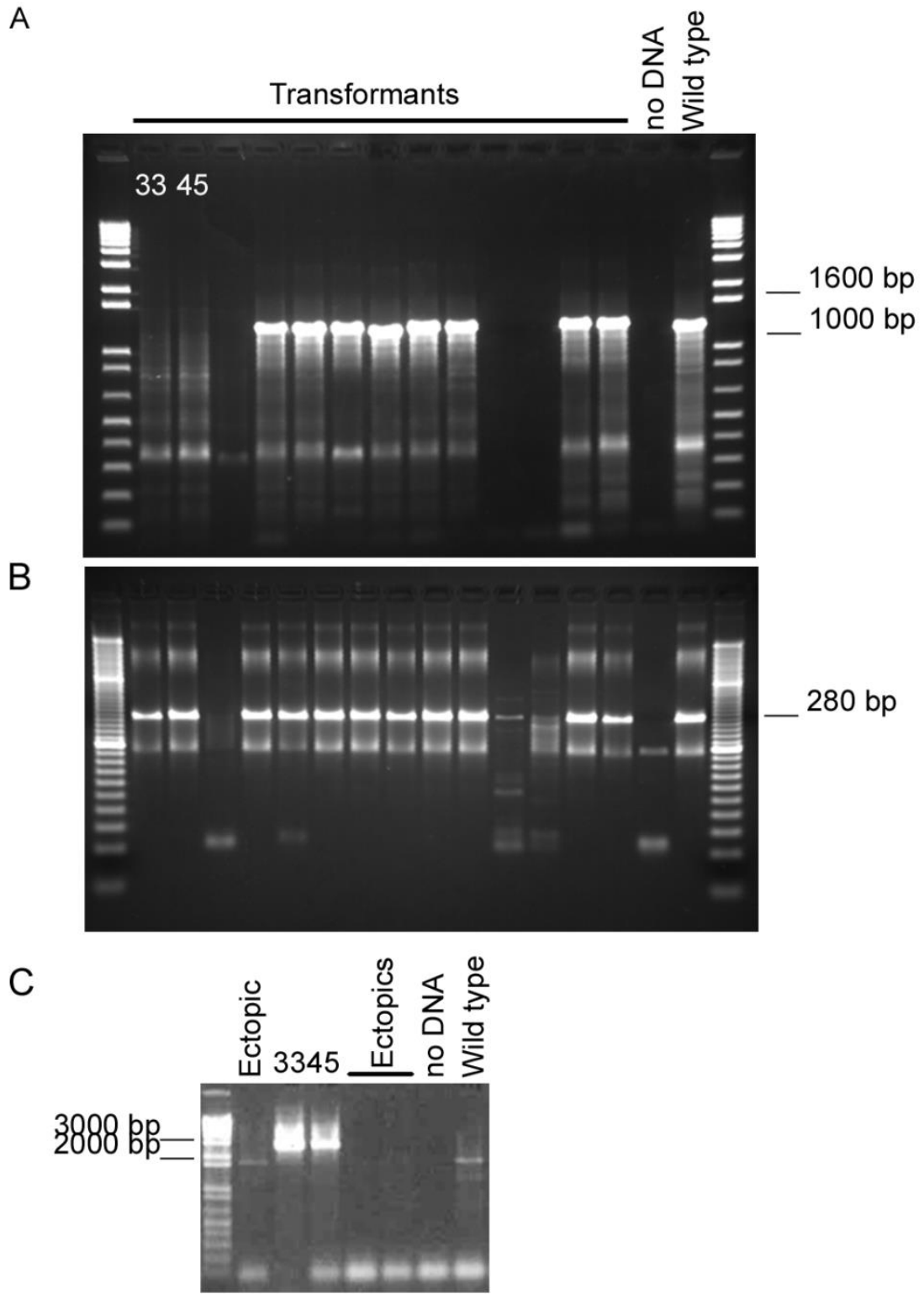

Figure 6. Disruption of Pfku70 in Pseudocercospora fijiensis CIRAD86. A) Selection of transformants by selective PCR resulting in amplification of the $P f k u 70$ region of $1,261 \mathrm{bp}$ in ectopic transformants. No amplicon was produced in potential transformants. B) Transformants 33 and 45 also amplified the same VNTR1333 allele as the wt, representing the only obtained knock-out strains. C) Candidate transformants 33 and 45 were confirmed as knock out since PCR analyses resulted in the expected and diagnostic 2,853 bp amplicon. 


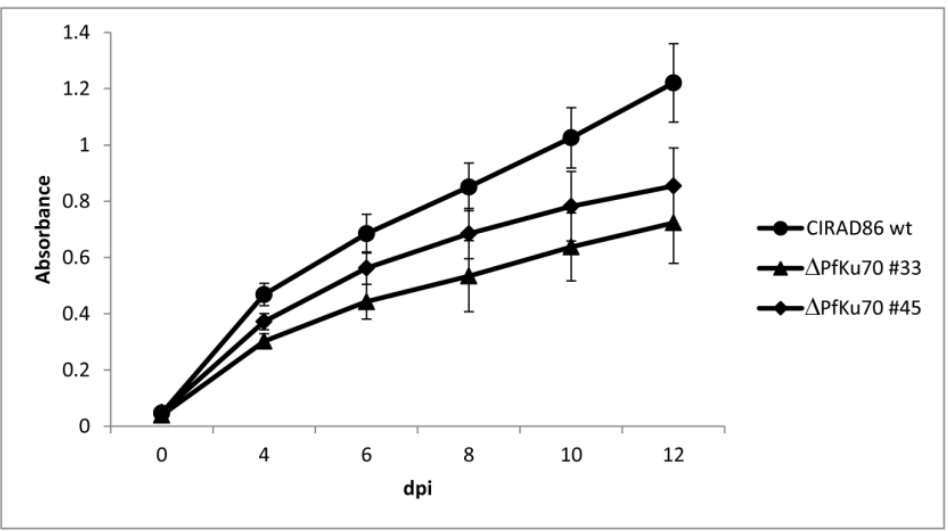

Figure 7. Comparison of mycelial growth of the Pseudocercospora fijiensis wt CIRAD86 strain and the knock-out strains $\triangle P f k u 70 \# 33$ and $\triangle P f k u 70 \# 45$. Absorbance $(690 \mathrm{~nm})$ reads of cultures in PDB medium were measured over time, averages of five reads over 21 positions in each of two biological and technical replicates per strain. 
Table 1. Transformation efficiency of Pseudocercospora fijiensis for random and targeted integration using Pfavr4, Pfku70 and the Pfcyp5Ipromoter.

\begin{tabular}{|c|c|c|c|c|c|c|c|c|}
\hline $\begin{array}{c}\text { Gene / } \\
\text { sequence }\end{array}$ & Mutagenesis & $\begin{array}{l}\text { Marker } \\
\text { gene }\end{array}$ & Strain & Transformants* & $\begin{array}{l}\text { Transformation } \\
\text { efficiency (\%) }\end{array}$ & $\begin{array}{l}\text { Hom. } \\
\text { Rec. }\end{array}$ & $\begin{array}{c}\text { Hom. Rec. } \\
\text { efficiency } \\
(\%)\end{array}$ & Reference \\
\hline eGFP & Random & hph & CIRAD86 & 20 & 0,0006 & n.a. & n.a. & This study \\
\hline $\begin{array}{l}\text { eGFP } \\
\text { DsRed }\end{array}$ & Random & hph & CIRAD139a & 6 & 0,0002 & n.a. & n.a. & This study \\
\hline $\begin{array}{l}\text { express } \\
\text { DsRed }\end{array}$ & Random & hph & CIRAD86 & 34 & 0,0011 & n.a. & n.a. & This study \\
\hline express & Random & hph & CIRAD139a & 2 & 0,00006 & n.a. & n.a. & This study \\
\hline Pfavr4 & Targeted & hph & CIRAD86 & 90 & 0,022 & 1 & 1,11 & This study \\
\hline $\begin{array}{l}\text { Pfku70 } \\
\text { Pfcyp51 }\end{array}$ & Targeted & hph & CIRAD86 & 102 & 0,025 & 2 & 1,96 & $\begin{array}{l}\text { This study } \\
\text { Diaz-Trujillo et }\end{array}$ \\
\hline
\end{tabular}

* Random transformants number correspond to the average from two experiments n.a. : not

applicable 
Table 2. Comparison of protein identity of PfKU70 with other fungal Ku70 proteins.

\begin{tabular}{lccccc}
\hline \multicolumn{1}{c}{ Specie } & Class & Accession & ID (\%) & Score & Reference \\
\hline Neurospora crassa & Sordariomycete & BAP16622.1 & 49.9 & 2.3 E-144 & Ninomiya et al., 2004 \\
Rhodosporidium toruloides & Mycrobotryomycete & AlA21643.1 & 39.6 & 3.84 E042 & Koh et al., 2014 \\
Zymoseptoria tritici & Dothideomycete & EGP88672.1 & 69.4 & 0 & Bowler et al., 2010 \\
Alternaria alternata & Dothideomycete & ADQ73897 & 57.2 & 0 & Wang et al., 2011 \\
Parastagonospora nodorum & Dothideomycete & EAT79812.2 & 56.6 & 0 & Feng et al., 2012 \\
Sphaerulina musiva & Dothideomycete & EMF13337 & 74.2 & 0 & Unpubished \\
\hline
\end{tabular}


Table 3. Primers used in this study.

\begin{tabular}{|c|c|c|}
\hline Primer name & Sequence & Reference \\
\hline Avr4_F1 & CATCATGCTTTCAACTACGAG & This work \\
\hline Avr4_R1 & GTTGTCGCATCCTGTTTC & This work \\
\hline Avr4_5'fwd & GGGGACAACTTTGTATAGAAAAGTTGGCCTTTTGACAGTATTCTCGTCAGCC & This work \\
\hline Avr4_5'rev & GGGGACTGCTTTTTTGTACAAACTTGCCCCTAGCGTTGCCGAATACGA & This work \\
\hline Avr4_3'fwd & GGGGACAGCTTTCTTGTACAAAGTGGCCAGCGGACGCTTCTTATAC & This work \\
\hline Avr4_3'rev & GGGGACAACTTTGTATAATAAAGTTTACCGAAGGCGTAGGTTGTC & This work \\
\hline Avr4-HR-F & CAGGGGGTGAAGAATGTGAG & This work \\
\hline Avr4-HR-R & ACTGTCGGGCGTACACAAAT & This work \\
\hline KU70_F1 & GAGCAAATTCCAGGAGCAAG & This work \\
\hline KU70_R1 & GTCAAACAGACCTCGGCAAT & This work \\
\hline KU70_5'fwd & GGGGACAACTTTGTATAGAAAAGTTGTGCATGGAGTGGAGTCAG & This work \\
\hline KU70_5_rev & GGGGACTGCTTTTTTGTACAAACTTGTCGTTTATGGAGGCGTAGT & This work \\
\hline KU70_3'fwd & GGGGACAGCTTTCTTGTACAAAGTGGTCTCGTCGAACTGGTCAC & This work \\
\hline KU70_3'rev & GGGGACAACTTTGTATAATAAAGTTGTCCAGGCTGATCGTTATA & This work \\
\hline KU70-HR-F & TCGTTCAAACATTTGGCAATAA & This work \\
\hline KU70-HR-R & AAATGCAGAGGCGTGGTATC & This work \\
\hline VNTR1333-F & GAGTGAAGTACTGCGGAGGC & Garcia et al., 2010 \\
\hline VNTR1333-R & AGTTGGAGAAAGGCGAAAGG & García et al., 2010 \\
\hline M13-F (-21) & TGTAAAACGACGGCCAGT & Common known \\
\hline M13-R & CAGGAAACAGCTATGACC & Common known \\
\hline
\end{tabular}


CHAPTER 3 


\section{CHAPTER 4}

A NEW MECHANISM FOR REDUCED SENSITIVITY

TO DEMETHYLATION-INHIBITOR FUNGICIDES IN

THE FUNGAL BANANA BLACK SIGATOKA

PATHOGEN PSEUDOCERCOSPORA FIJIENSIS

Diaz-Trujillo, C., Chong, P., Stergiopoulos, I., Cordovez, V., Guzman, M., De Wit, P.J.G.M., Meijer, H.J.G., Scalliet, G., Sierotzki, H., Lilia Peralta, E., Arango Isaza, R.E., Kema, G.H.J., 2018. A new mechanism for reduced sensitivity to demethylation-inhibitor fungicides in the fungal banana black Sigatoka pathogen Pseudocercospora fijiensis. Mol. Plant Pathol. https://doi.org/10.1111/mpp.12637 


\section{Abstract}

The Dothideomycete Pseudocercospora fijiensis, previously Mycosphaerella fijiensis, is the causal agent of black Sigatoka, one of the most destructive diseases of bananas and plantains. Disease management depends on fungicide applications with a major share for sterol demethylation-inhibitors (DMIs). The continued use of DMIs puts a considerable selection pressure on natural P. fijiensis populations enabling the selection of novel genotypes with reduced sensitivity. The hitherto explanatory mechanism for this reduced sensitivity was the presence of non-synonymous point mutations in the target gene Pfcyp51, encoding the sterol 14 $\alpha$-demethylase enzyme. Here, we demonstrate a second mechanism involved in DMI sensitivity of $P$. fijiensis. We identified a $19 \mathrm{bp}$ element in the wild type (wt) Pfcyp51 promoter that concatenates in strains with reduced DMI sensitivity. A PCR assay identified up to six $P f c y p 51$ promoter repeats in four field populations of P. fijiensis in Costa Rica. We used transformation experiments to swap the wild type promoter of a sensitive field isolate with a promoter from a strain with reduced DMI sensitivity that comprised multiple insertions. Comparative in vivo phenotyping showed a functional and proportional upregulation of Pfcyp51, which consequently decreased DMI sensitivity. Our data demonstrate that point mutations in the Pfcyp51 coding domain as well as promoter inserts contribute to reduced DMI sensitivity of $P$. fijiensis. These results bring new insights into the importance of the appropriate use of DMIs and the need for the discovery of new molecules for black Sigatoka management.

Keywords: Fungicide, DMI, Pfcyp51 promoter. 


\section{Introduction}

Black Sigatoka, caused by the ascomycete Pseudocercospora fijiensis (Morelet, 1969) Deighton (1976), (previously Mycosphaerella fijiensis Morelet (1969), is one of the most devastating and economically significant diseases of export bananas and plantains. Disease management is mainly based on the extensive application of primarily single-site fungicides. However, the continuous sexual reproduction of $P$. fijiensis generates genetically highly diverse and hence, versatile populations that quickly adapt to changing environments including extensive fungicide treatments (Arango et al., 2016; Conde-Ferráez et al., 2007; Hayden and Carlier, 2003; Rivas et al., 2004; Romero and Sutton, 1997). As a result, reduced fungicide efficacy develops frequently and spreads rapidly (Arango et al., 2016). This situation has contributed to a grave increase in the number of fungicide applications, which can tally up to over 50 applications per year (maximally 10 applications with sterol $14 \alpha-$ demethylation inhibitors, DMIs) in most banana export countries (Chong, 2016; De Lapeyre De Bellaire et al., 2010; FRAC, 2010; Martínez-Bolaños et al., 2012), thereby frequently comprising a 30\% share of the production costs (Marín et al., 2003). This practice poses a threat on the occupational health of plantation workers, and the environment, if guidelines are not followed. It is thus imperative to understand the mechanisms by which reduced fungicide efficacy develops to enable adequate long-term disease management strategies with optimized chemical input.

Azole fungicide applications against black Sigatoka started in 1987 and became widely used since 1991 when propiconazole, one of the major contemporary DMIs, was introduced in the market (Chong, 2016; Romero and Sutton, 1997). Currently, several DMIs, such as difenoconazole, bitertanol, and epoxiconazole are used in disease management programs, either alone or in mixes with other fungicides with different modes of action. DMIs inhibit the activity of the CYP51 enzyme that is involved in the $14 \alpha$-demethylation of 
the ergosterol precursor eburicol (24-methylene-24, 25-dihydrolanosterol). Ergosterol regulates cellular membranes fluidity and permeability and is essential for cell viability (Lepesheva and Waterman, 2011). However, reduced efficacy of single-site fungicides surfaced rapidly in $P$. fijiensis after the introduction of quinone outside inhibitors (QoIs or strobilurins), methyl benzimidazole carbamates (MBCs), and DMIs for disease control in banana production (Arango et al., 2016; Amil et al., 2007; Cañas-Gutiérrez et al., 2009, 2006; Romero and Sutton, 1997). Previous studies on P. fijiensis revealed the correlation between reduced efficacy of propiconazole and point mutations in the coding domain of the Pfcyp51 gene, which caused non-synonymous amino acid (aa) substitutions surrounding the Substrate Recognition Sites (SRS) at positions Y136, A313, Y461 and Y463 (Cañas-Gutiérrez et al., 2009; Chong, 2016). Until now, this was the only explanatory mechanism for reduced sensitivity towards azoles in $P$. fijiensis. Here, we introduce an additional mechanism that drives reduced sensitivity to DMIs in P. fijiensis. We identified the presence of one or more repetitive elements in the promoter region of Pfcyp51 among P. fijiensis field isolates with reduced DMI sensitivity and catalogued such variants in 225 field isolates originating from various - treated and untreated - banana plantations in Costa Rica. Comparison with 14 control isolates from Ecuador, Asia and Africa showed a positive correlation between the presence and copy number of the Pfcyp51 promoter elements, Pfcyp51 overexpression and reduced DMI sensitivity. We, subsequently, established the functional relationship between the number of promoter inserts, increased target expression and reduced DMI sensitivity through Pfcyp51 promoter swapping experiments between wild type (wt) isolates and $P$. fijiensis strains with reduced DMI sensitivity. We thereby formally demonstrated a novel mechanism involved in reduced fungicide efficacy of DMIs to P. fijiensis, in addition to the described target site mutations in the coding sequence of Pfcyp51. 


\section{Results}

\section{In vitro sensitivity to propiconazole}

The $P$. fijiensis isolates that were tested for sensitivity to propiconazole were classified in three groups; sensitive isolates with (1) $\mathrm{EC}_{50}$ values of $\leq 0.10 \mathrm{mg} . \mathrm{L}^{-1}$; (2) moderately resistant isolates with $\mathrm{EC}_{50}$ values between 0.10 to $1.0 \mathrm{mg} . \mathrm{L}^{-1}$ and (3) resistant isolates with $\mathrm{EC}_{50}$ values $>1.0 \mathrm{mg} \cdot \mathrm{L}^{-1}$ (Table 1). Among the 25 isolates tested for sensitivity to propiconazole, seven were sensitive, 14 moderately resistant and four were resistant. Clear cross-resistance between propiconazole and cyproconazole was observed, since the majority of isolates showed similar $\mathrm{EC}_{50}$ values (Table 1, Figure $\mathrm{S} 1$ ).

\section{Pseudocercospora fijiensis isolates with reduced sensitivity always contain repetitive elements in the Pfcyp51 promoter}

Detailed comparison between the Pfcyp51 promoter sequences from resistant isolates and the reference $P$. fijiensis isolate CIRAD86 revealed that resistant isolates possess an insertion in the promoter at 103bp upstream from the start codon. Meanwhile, some isolates with reduced sensitivity showed a shorter insertion than resistant strains at the same position. Likewise, sensitive isolates did not show any insertion. Insertions comprise repeats of $19 \mathrm{bp}$ elements "TAAATCTCGTACGATAGCA" present once in the Pfcyp51 promoter 122bp upstream from start codon, at scaffold 7:2121794 - 2121813 of the CIRAD86 reference (Pseudocercospora fijiensis v2.0, JGI) (Figures 1 and 2).

Some isolates contain part of the element in their insertions, while others have a modified element due to a few additional nucleotides. Additional to the $19 \mathrm{bp}$ element, a slightly modified $16 \mathrm{bp}$ (TAAAATCTCGTACGAT) and a 20bp (TAAAATCTCGTACGATAGCA) were also present in the Pfcyp51 promoter. For example, in resistant isolates Ca1_5, Ca5_16, Ca6_11, and Ca10_13 (Table 1; S Text) the basic 19bp 
element is repeated up to six times (four fully conserved and one partial, mostly in tandem insertion) and thrice in the moderately resistant $P$. fijiensis isolates Z8_12 and Z8_18. DNA sequence analysis of the resistant isolates from Costa Rica (Ca5_16, Ca6_11 and Ca10_13), revealed that these contain identical mutations in the coding region of the Pfcyp51 gene, and that the overall length of the Pfcyp51 promoter inserts accumulates to 100bp (Table 1).

\section{Repetitive elements in the promoter of Pfcyp51 upregulate its expression}

To test whether Pfcyp51 gene expression is affected by the presence of repetitive elements, we quantified the expression in mycelium by real time RT-PCR, normalized to the expression of the actin gene (Pfact) as compared to wild type (wt) controls. P. fijiensis isolates Ca5_16, Ca6_11 and Ca10_13, all containing six repeat elements in the Pfcyp51 promoter, showed a 3.3-5.6 fold increase in Pfcyp51 gene expression as compared to control isolate E22, and a smaller difference to the other control strain CIRAD86 that only have the basic 19bp element (Figure 3). In contrast, no significant difference was found between the control isolate CIRAD86 and P. fijiensis isolate Z8_12, which has three repeat elements. The up-regulation of $P f c y p 51$ was constitutive and independent of addition of propiconazole in the culture medium (data not shown).

Pfcyp51 promoter insertions accumulate in $P$. fijiensis strains with reduced fungicide sensitivity originating from frequently sprayed commercial banana plantations in Costa Rica

To identify the number of repeat element copies in the Pfcyp51 promoter, we performed PCR analyses on 225 isolates originating from four banana plantations in Costa Rica that were previously studied (Arango et al., 2016): three plantations (Cartagena, Zent 
and San Pablo) with intensive fungicide applications and one unsprayed plantation (ZTSC or San Carlos). Comparison of the amplicon sizes by gel electrophoresis and sequence data revealed banding patterns that corresponded to two, three and six promoter repeats (Figure 4).

Isolates containing six repeat elements dominated (50 out of 82) the Cartagena population, followed by isolates with two copies ( 29 out of 82 ), whereas isolates with merely the original $19 \mathrm{bp}$ element were scarce (3 out of 82 ). In contrast, the Zent population was dominated by isolates with only the $19 \mathrm{bp}$ element in the Pfcyp51 promoter (59 out of 84), but isolates containing two and six promoter repeats were also found (11 and 14 out of 84, respectively). The San Pablo population was dominated (23 out of 43) by a genotype with three promoter repeats that was not observed in the other populations in addition to strains with one ( 8 out of 23$)$ and two (2 out of 23$)$ promoter repeats. None of the genotypes with accumulated promoter repeats were observed in the San Carlos populations that exclusively comprised $P$. fijiensis strains with the original $19 \mathrm{bp}$ element in the Pfcyp51 promoter (Figure 4).

Sequence analyses revealed that the accumulated promoter repeat elements varied from 42bp (two elements), 59bp (three elements) up to 100bp (six elements). All repeat elements are inserted exactly $103 \mathrm{bp}$ upstream of the start codon of $P f c y p 51$ and are either 20bp (TAAAATCTCGTACGATAGCA), 19bp (TAAATCTCGTACGATAGCA) or 16bp (TAAAATCTCGTACGAT) in length and concatenate in tandem or are separated by a few nucleotides. Elements of $20 \mathrm{bp}$ and $19 \mathrm{bp}$ only differ by one extra adenine, whereas the 16bp element represents a shorter version of the 19bp insert (Figure 1). The 19bp element was found in isolates with one, two and three copies, whereas in isolates with six Pfcyp51 promoter inserts the 19bp element was always accompanied by single inserts of the 16bp and 20bp units. Hence, the 19bp element is the commonest insertion across all isolates analysed (Figure 1). 


\section{Analysis of the Pfcyp51 coding sequence}

As expected, sequence analyses of different isolates revealed the presence of nonsynonymous mutations in the coding region of Pfcyp51. These resulted in aa changes Y136F, A313G, Y463D/H/N that were previously reported and associated with reduced sensitivity to propiconazole (Cañas-Gutiérrez et al., 2009). Here, we identified nine new aa substitutions (T18I, Y58F, V106D, V116L, K171R, A381G, A446S, G462A, and Y463S) (Table 1). All isolates contained the T18I and V106D substitutions. Apart from these, the most frequent aa substitutions $\mathrm{A} 313 \mathrm{G}$ and $\mathrm{Y} 463 \mathrm{~N} / \mathrm{D} / \mathrm{S} / \mathrm{H}$ were observed in 11 and 16 out of 25 isolates, respectively. These mutations were often found in combination with Y136F and A381G. Thus, the most frequently observed haplotypes amongst the 25 isolates were T18I, V106D, Y136F, A313G, Y463D/N/S, which were found in combination with two, three or six copies of the Pfcyp51 repeat element. Strains with the T18I, V106D, Y136F and Y463D Pfcyp51 modifications showed the least sensitivity to the tested fungicides. In addition, several other combinations of aa substitutions were observed in the analysed cohort of $P$. fijiensis isolates, including A313G and Y463S/H/D/N; A381G and G462A; Y136F and Y463D; Y136F, A381G and Y463D; and K171R and A446S.

\section{Functional analysis of the Pfcyp51 promoter insertions}

We discovered a range of promoter insertions in $P$. fijiensis isolates from banana plantations that were treated with fungicides. These promoter insertions, in particular the six repeat inserts, conferred enhanced expression of Pfcyp51. The isolates carrying these insertions also displayed reduced sensitivity to DMI fungicides, but also carried Pfcyp51 mutations in the coding sequence, which is the hitherto only explanatory mechanism for reduced DMI sensitivity. To disentangle the relation between mutations in the coding 
sequence and the promoter insertions, we introduced the Pfcyp51 promoter from the resistant P. fijiensis isolate Ca5_16 with six repeat elements into the sensitive wt E22 isolate from Ecuador (Table 1; Figure 5).

Transformation of wt $P$. fijiensis isolate E22 resulted in 250 green fluorescent protein (GFP) and hygromycine ( $h g h)$ positive transformants. The transformants were characterized by PCR to differentiate isolates with six repeats in the Pfcyp51 promoter at the correct integration site from ectopic transformants (Figure 5). Two independent transformants, Swap26 and Swap121, showing the Ca5_16 promoter amplicon and positive for the correct integration site were selected for further analyses (Figure 5). Subsequently, we performed qRT-PCR analyses on Swap26 and Swap121 along with the P. fijiensis control isolates comprising the recipient wt isolate E22 and the wt resistant isolates Ca5_16 and Ca10_13 and an ectopic transformant. Consistent with previous results, the resistant isolates Ca5_16 and Ca10_13 express Pfcyp51 at a higher level than the wt E22 recipient isolate. Moreover, the expression of Pfcyp51 was significantly increased in both Swap26 and Swap121 compared to wt strain E22 and the ectopic isolate, and not significantly different from the resistant donor isolate Ca5_16 (Figure 6). Hence, these results prove that replacing the Pfcyp51 promoter from a sensitive $P$. fijiensis isolate by the promoter from a resistant strain results in over expression of Pfcyp51.

To determine whether the observed effect was independent of azole fungicides we challenged the transformants with difenoconazole, epoxiconazole and propiconazole in 96well plates and calculated the $\mathrm{EC}_{50}$ values. A consistent growth pattern was observed for all controls $\left(0 \mathrm{mg} \cdot \mathrm{L}^{-1}\right)$. Wt strain Ca10_13 grew up to $2.56 \mathrm{mg} \cdot \mathrm{L}^{-1}$ of difenoconazole or epoxiconazole, and $10.24 \mathrm{mg} \cdot \mathrm{L}^{-1}$ of propiconazole (wt isolate Ca5_16 was removed due to contamination). The sensitive wt isolate E22 and the ectopic transformant only grew up to $0.016 \mathrm{mg} \cdot \mathrm{L}^{-1}$ of difenoconazole and $0.04 \mathrm{mg} \cdot \mathrm{L}^{-1}$ of epoxiconazole or propiconazole. The 
Swap26 and Swap121 transformants grew on DMI concentrations that were at least fourfold higher than those of the sensitive wt control isolate E22. For difenoconazole, transformants Swap26 and Swap121 displayed a twofold and over fourfold $(4,25)$ increment of $\mathrm{EC}_{50}$ compared to the sensitive wt check E22, respectively (Figure 6). For epoxiconazole, Swap26 displayed a 4.48-fold reduction in sensitivity, while Swap121 displayed a slightly higher 8.36fold reduction. Finally, the $\mathrm{EC}_{50}$ for propiconazole of the wt strain $\mathrm{E} 22$ was 4.65 -fold and 5.23-fold lower compared to Swap26 and Swap121, respectively. The ectopic transformant, displayed a similar sensitivity as wt E22 regardless of the fungicide used (Figure 6). These data confirm that $P f c y p 51$ promoter modifications contribute to reduced DMI efficacy in $P$. fijiensis. 


\section{Discussion}

Disease management in agricultural crops is commonly based on an integrated approach comprising host resistance, agronomic measures and crop protection agents whenever necessary (Matthews et al., 2014). Due to the ubiquity of "Cavendish" clones, which represent over $90 \%$ of the global banana trade, and their vulnerability to P. fijiensis, disease control in banana almost entirely relies on crop protection agents and prophylaxis measures. Despite the use of decision support systems accompanied with leaf surgery and the removal of infected foliage to reduce the inoculum potential, the cornerstone for $P$. fijiensis control remains chemical crop protection, with the emphasis on azole fungicides (Price et al, 2015). Consequently, the selection pressure on the pathogen has been enormous that resulted in the appearance of $P$. fijiensis populations with reduced fungicide sensitivity, which calls for a better understanding of its origin and dissemination.

The presence of mutations in the Pfcyp51 gene has been previously related to propiconazole resistance in P. fijiensis (Cañas-Gutiérrez et al., 2009). Here, we have focused on the promoter region as an important determinant for $P f c y p 51$ gene expression, and describe the identification of a $19 \mathrm{bp}$ element, whose concatenation upregulates $P f c y p 51$ expression and confers reduced DMI sensitivity. Our data represent the first report of targeted genetic modification of $P$. fijiensis to demonstrate a new mechanism for DMI sensitivity modulation in this organism.

PfCYP51 substitutions Y136F, A313G, A381G, Y461D, Y463D, Y463H and Y463N were found in the present study in accordance to what has been previously described for $P$. fijiensis for propiconazole (Cañas-Gutiérrez et al., 2009) as well as to other azoles in Zymoseptoria tritici, Candida albicans, Pyrenophora teres f. sp. teres, and Aspergillus fumigatus (Akins and Sobel, 2009; Cools and Fraaije, 2013; Mair et al., 2016; Mellado et al., 
2007). Unexpectedly, we identified a 100bp insertion in the Pfcyp51 promoter region in addition to the coding region mutations in most $P$. fijiensis isolates from the Cartagena population. These insertions comprise six copies of a repetitive element, whereas a single copy of this element is present in all sensitive isolates. Isolates with reduced sensitivity have usually two, three or more copies of this element (Chong, 2016).

Unlike in P. teres f. sp. teres (Mair et al., 2016) and Erysiphe necator (Rallos and Baudoin, 2016) which showed overexpression of Cyp51, but no promoter modification, changes in the promoter region of the cyp51 gene have been described in other fungi. Such changes comprise repeated promoter elements, truncated derivatives of a LINE-like retrotransposon in Blumeriella jaappi (Ma et al., 2006), a MITE-like transposon named PdMLE1 in Penicillium digitatum (Sun et al., 2013), or a larger transposon of $1.8 \mathrm{~kb}$ in $A$. fumigatus (Albarrag et al., 2011; Verweij et al., 2013), or transcription factor binding sites in Venturia inaequalis (Villani et al., 2016). More detailed studies would be required in $P$. fijiensis to decipher whether the repeat elements that we observed correspond to the movement of a transposon sequence or whether Pfcyp51 expression is possibly co-regulated by transposons. However, unlike previous reports of promoter insertions with $199 \mathrm{bp}$ to 5.6 kbp-sequence transposons in V. inaequalis (Schnabel and Jones, 2001; Villani et al., 2016), the Pfcyp51 promoter insertion merely comprise 19bp elements, or minor 16bp and 20bp variants, which accumulate up to $100 \mathrm{bp}$ in length, shorter than insertions in $\mathrm{V}$. inaequalis, and Z. tritici (Cools et al., 2012), where no transposons were reported. Thus the insertions in the Pfcyp51 promoter are shorter than any promoter insertions reported in A. fumigatus (Snelders et al., 2012; Verweij et al., 2007), and Pyrenopeziza brassicae (Carter et al., 2014). In other organisms, e.g. Escherichia coli, overexpression of a desired gene was achieved by tandem repeats of core promoter sequences called "MCPtacs" (Li et al., 2012). In this way, a higher number of mutations in the coding region could be controlled, which would compromise the 
activity of the enzyme and hence to reduced sensitivity (Cools et al. 2012; Leroux \& Walker 2011). Possibly, this also applies to P. fijiensis, as we did not find strains with reduced sensitivity and insertions in the promoter, but no mutations in the coding region. Isolates from wt populations lacked promoter insertions, but - occasionally - possessed mutations in the coding region.

We studied the regulatory nature of the inserted sequences in P. fijiensis in silico and showed that the 19bp (TAAATCTCGTACGATAGCA) repeat element is the commonest feature. Within populations, we identified a clear genetic diversity in the number of promoter repeats. The frequency of isolates with more repeats was higher in banana plantations with up to eight DMI cycles, such as Cartagena, Zent and San Pablo. Although expected, it is also striking that all isolates from the untreated San Carlos plantation contained the single 19bp element. Using a targeted reverse genetics approach in $P$. fijiensis we, for the first time, could validate that the presence of six copies of this element in the promoter increases the expression of $P f c y p 51$ at least three-fold compared with wt isolates and others with reduced sensitivity and up to three repeat elements. Previously, Cañas-Gutiérrez et al. (2009) were unable to show such expression in experiments with $P$. fijiensis in response to propiconazole and considered it either a non-existent or unimportant mechanism in this fungus. However, this was likely due to the use of fewer isolates that showed a limited reduction of sensitivity. Hence, we now propose that promoter repeats constitute a genetic adaptation mechanism to the high selective pressure imposed on P. fijiensis by the continuous use of different DMI fungicides.

Even though $P$. fijiensis is a difficult fungus to transform (chapter 3), and despite that site specific recombination levels seem to be very low, promoter swapping was successfully applied in our study. The introduction of the promoter from a $P$. fijiensis isolate with strongly reduced sensitivity into a sensitive isolate by site specific recombination resulted in a 
transformant with increased expression of $P f c y p 51$, and consequently reduced sensitivity to three azole fungicides, as a result of the promoter replacement. The Swap26 and Swap121 transformants were at least four times less sensitive than the recipient wt isolate E22, but not as resistant as the wt resistant isolate Ca10_13 or the donor wt isolate Ca5_16, which had similar (Y136F and Y463D) coding domain mutations. Hence, we expect that the reverse experiment, replacing the wt promoter (with inserts) from an isolate with reduced sensitivity with a promoter from a sensitive wt should result in an increase of sensitivity. Finally, swapping the wt Pfcyp51 coding domain of a sensitive strain with this domain of an isolate with reduced sensitivity, thereby generating a strain with a wt coding domain, but multiple promoter inserts, which we have never encountered in nature, should result in increased sensitivity. However, discovery of additional mechanisms for DMI sensitivity require genetic studies, either genome wide associations or mapping analyses (Chong, 2016). We expect, however, that the combination of overexpression conferred by promoter insertions and Pfcyp51 target site mutations explain most DMI sensitivity modulations.

DMIs are and will likely remain a cornerstone for global black Sigatoka disease management. However, the risks of bad practices or excessive applications exert a significant selection pressure on P. fijiensis populations, turning these increasingly insensitive. Hence, DMI applications may lose their competitive advantage compared to other less environmentally friendly compounds. The practical spin-off of this study is that we can now use a simple PCR assay to monitor, evaluate and predict reduced DMI sensitivity in $P$. fijiensis field populations. Albeit that we focus here on P. fijiensis, DMIs are evidently under pressure due to overall reduced sensitivity issues (Chen et al., 2016; Hayashi et al., 2002; Leroux and Walker, 2011; Liu et al., 2015; Mullins et al., 2011; Sun et al., 2014, 2013; Villani et al., 2016) and are, therefore, increasingly studied in various other fungal pathogens (Alvarez-Rueda et al., 2011; Becher and Wirsel, 2012; Carter et al., 2014; Cools et al., 2012; 
Frenkel et al., 2014; Li et al., 2012; Luo and Schnabel, 2008; Maier et al., 2016; Nikou et al., 2009; Rallos and Baudoin, 2016; Verweij et al., 2013). This fosters research and development for novel chemistry for efficient black Sigatoka control, although alternative products, such as the succinate dehydrogenase inhibitors (SDHIs) and QoIs, are also prone to resistance development (Arango et al., 2016; Scalliet et al., 2012). Therefore, disease management should on the long run embark on the availability of resistant banana germplasm. As this will take years, fungicide sensitivity monitoring and the strict adoption of application recommendations remain absolute necessities, irrespective of which banana cultivars dominate the export trade. A more science driven disease management and extension practice in global banana production is the prerequisite for a continuous production of this global top fruit and major staple food. 


\section{Material and methods}

\section{Pseudocercospora fijiensis isolates}

A set of 25 monoascosporic P. fijiensis isolates from Africa, Asia and Latin America, was used for fungicide sensitivity assays. Eight of the Latin-American isolates were collected in Ecuador and 11 isolates in Costa Rica (see Table 1). The larger set of Costa Rican isolates originated from four different banana plantations: Cartagena (Ca), Zent (Z), San Pablo (SP) and San Carlos (ZTSC) (see also Arango et al., 2016). The former three are frequently sprayed with fungicides, whereas the San Carlos plantation is in a plantain growing area with low $P$. fijiensis incidence, hence fungicides are not required for disease control. We consider the $P$. fijiensis population from this area as a wt population. Isolates were obtained from CORBANA (Costa Rica), CIBE-ESPOL (Ecuador) and the Westerdijk Fungal Biodiversity Institute (Africa and Asia).

\section{Determining the in vitro sensitivity to DMI fungicides}

The fungicides propiconazole, cyproconazole and difenoconazole were provided by Syngenta (Syngenta Crop Protection AG, Basel, Switzerland) and epoxiconazole was obtained from Sigma (Sigma Aldrich, Missouri, USA). All compounds were technical grade quality and were maintained in 100x stock solutions, either in methanol or DMSO. When applied to the culture medium the final concentration of the solvents was $<1 \%(\mathrm{v} / \mathrm{v})$. For the initial in vitro sensitivity assays the final concentrations tested for propiconazole were 10 , $5.62,3.16,1.78,1.0,0.56$, and $0.31 \mathrm{mg} \cdot \mathrm{L}^{-1}$. Subsequently, to evaluate sensitive isolates more accurately, lower concentrations of fungicides were included in the assays $(10.24,2.56,0.64$, $0.16,0.04,0.016,0.004,0 \mathrm{mg} \cdot \mathrm{L}^{-1}$ ) and exploited to evaluate the performance of $P$. fijiensis transformants in the presence of propiconazole, difenoconazole and epoxiconazole. 
Fungicide sensitivity of each isolate was determined by calculating the $50 \%$ inhibitory concentration $\left(\mathrm{EC}_{50}\right)$. Quantitative analysis of fungal growth, was determined by a modified 96 -well microtiter plate dilution assay (Montoya et al., 2006). Fifty microliters of a $1 \times 10^{5}$ mycelial parts $\cdot \mathrm{mL}^{-1}$ solution from each isolate were inoculated in $200 \mu 1$ potato dextrose broth (PDB) medium per well of a 96-well polystyrene, flat bottom, transparent, plate (Corning, USA; cat. \# 3370). Plates were incubated at $25^{\circ} \mathrm{C}$ in an incubator (Elbanton, Kerkdriel, Netherlands) for seven days before mycelial growth was measured. Each concentration was tested in duplicate per isolate, and per plate four blank controls were present. Individual plates were considered as one biological replicate, and tests were performed thrice. Absorbance was initially measured at $620 \mathrm{~nm}$ in a TECAN A5082 plate reader (Männedorf, Switzerland), but due to the variation of mycelial colours over the isolates as well as the different colony morphologies, we eventually monitored growth at an absorbance of $690 \mathrm{~nm}$ in an Infinite ${ }^{\circledR}$ M200 PRO reader (TECAN, Männedorf, Switzerland), which enabled measuring higher sensitivities. The read design per well was settled at room temperature, leaving a border of $1,000 \mu \mathrm{m}$, a bandwidth of $9 \mu \mathrm{m}$, circle-filled reads of $21 \mathrm{read}$ points $(5 \times 5$, with no corner points for circle distribution), and each read point was measured five times. Read averages were plotted against days after inoculation (dpi) and compared with the other isolates and controls. The fungicide sensitivity of transformants and control isolates was determined in the aforementioned 96-well polystyrene plates. Sealed plates were maintained at $27{ }^{\circ} \mathrm{C}$ in an incubator (Elbanton, Kerkdriel, Netherlands) in darkness and fungal growth was evaluated 10 dpi. Plates were evaluated at $690 \mathrm{~nm}$, while covered to reduce contamination. Data were analysed using GraphPad Prism7 (GraphPad Software, La Jolla, USA). 


\section{Pfcyp51 coding domain and promoter amplification and sequencing}

To amplify the Pfcyp51 gene and the promoter region, specific primers located at the first repeat element and 22bp upstream of the open reading frame (ORF) were used: CYP51_Pfijien_F1 (5'-AAGGTCATATCGCAGG-3') and CYP51_Pfijien_R1 (5'GAATGTTATCGTGTGACA-3'). A basic PCR mix was prepared and the PCR program consisted of five min. of denaturation at $94{ }^{\circ} \mathrm{C}$ followed by 34 cycles of $30 \mathrm{sec}$. at $94{ }^{\circ} \mathrm{C}, 30$ sec. of annealing at $55{ }^{\circ} \mathrm{C}$ and 90 sec. of extension at $68{ }^{\circ} \mathrm{C}$. An additional extension step of seven min. at $72{ }^{\circ} \mathrm{C}$ was performed at the end. DNA sequencing of the gene was performed at Macrogen (Seoul, Korea) and by the Genomics facility of Wageningen University and Research (WUR), directly using the PCR products. To obtain the entire sequence of the gene and the promoter region four primers were used in the sequencing reactions: CYP51_Pfijien_F2 (5'-ACAGAAACATCACCTCC-3'), CYP51_Pfijien_F3 (5'ATTGCTTCACTTTCATCC-3'), CYP51_Pfijien_F4 (5'-CTCTACCACGATCTCGAC-3') and CYP51_Pfijien_R2 (5'-GATATGGATATAGTTGTC-3'). The obtained sequences were assembled in contigs per isolate using CLC DNA Workbench software (CLC bio, Aarhus, Denmark) and the ORF was translated to aa and the protein sequences were aligned using the ClustalW plug in. The sequence alignments allowed the identification of mutations.

\section{Pfcyp51 gene expression analysis}

Extraction of total RNA was carried out with mycelia of $P$. fijiensis isolates grown for 10 days in PDB using the Qiagen RNA extraction plus mini kit (QIAGEN Inc., Valencia, USA). The integrity of the RNA was checked using agarose gel electrophoresis and the concentration was determined by measuring absorbance at $260 \mathrm{~nm}$ in a Nanodrop spectrophotometer (Thermo scientific, Wilmington, USA). Expression analysis was 
performed by quantitative real time -PCR (qRT-PCR) using primers QRTCYP-forward: (5'CGCCAGTATTCGGCACAGATGTCG-3') and QRTCYP-reverse: (5'TAACGTAGGACTGGAGGGCGGA-3'), which amplify a fragment of 89bp of the Pfcyp51 gene and primers QRTACT-forward: (5'-TCCGTCCTTGGTCTCGAATCTGGT-3') and QRTACT-reverse: (5'-TGCATACGGTCGGAGATACCTGGA-3'), which amplify a fragment $146 \mathrm{bp}$ of the $P$. fijiensis actin gene that was used to normalize the expression. Quantitative RT-PCR reactions were performed using $20 \mathrm{ng}$ of total RNA per isolate in an Applied Biosystems ABI 7500 thermocycler (Waltham, USA) using the Applied Biosystems Power SYBR® Green RNA-to-CT ${ }^{\mathrm{TM}}$ 1-Step Kit, according to the manufactures instructions. The delta-delta $\mathrm{Ct}$ method was used - with the actin gene as the endogenous control - to determine the level of Pfcyp51 gene expression (Livak and Schmittgen, 2001).

\section{Analysis of promoter repeats of Pfcyp51 gene in four Costa Rican P. fijiensis populations}

Genomic DNA (gDNA) of 225 P. fijiensis isolates from the four Costa Rican populations was analysed; 82 from the Cartagena population, 43 from the San Pablo population, 84 from the Zent population, and 16 from the San Carlos wt population (Table S1). PCR fragments were amplified from gDNA using the specific primer pair, P._fijiensis_repeats_F (5'-TCTCGTACGATAGCACCTGCCCA-3') P._fijiensis_repeats_R (5'-TGTTGGTGTAGGGGGTTAGGCCA-3') that was designed to amplify the promoter region of Pfcyp51. PCR conditions comprised two min. at $95{ }^{\circ} \mathrm{C}, 30$ cycles of $30 \mathrm{sec}$. denaturation at $95{ }^{\circ} \mathrm{C}, 30 \mathrm{sec}$. of annealing at $68{ }^{\circ} \mathrm{C}$, and two min. of extension at $72{ }^{\circ} \mathrm{C}$ with an additional extension step of $10 \mathrm{~min}$. at $72{ }^{\circ} \mathrm{C}$ at the end of the reaction. PCR products were visualized and evaluated on $1 \%$ agarose gels and eleven isolates were selected for sequencing and subsequent analysis of promoter and coding sequences. 
Different repeat elements were aligned and a weblogo consensus sequence was generated (Crooks et al., 2004) to graph nucleotide conservation within the elements.

\section{Promoter swapping}

We performed a promoter swapping experiment to test the effect of promoter repeats on Pfcyp51 expression and henceforward on sensitivity to several azole fungicides. The Pfcyp51 donor promoter for homologous recombination was obtained from the resistant isolate Ca5_16. The recombination construct pPROM_CYP51_Ca5_16 comprised an upstream 2,024bp fragment (the PfCyp51 gene has an antisense position in the genome),

obtained by using primers 5-CYP-Prom Fwd (5'GGGGACAACTTTGTATAGAAAAGTTGAGGATATCAAGCACGCAC-3’) and Rev (5'GGGGACTGCTTTTTTGTACAAACTTGGAAGAGAAACGGACTCCA-3’), which was cloned in front of a cassette with the $h p h$ resistance gene and the GFP gene, followed by the upstream region of $1,737 \mathrm{bp}$ obtained with primers 3-CYP-Prom Fwd (5'GGGGACAGCTTtCTTGTACAAAGTGGGAATGAGCATTTGAGAGC-3') and Rev (5'GGGGACAACTTTGTATAATAAAGTTAATACTAGCGGAGGTTCG-3'), containing the promoter region of isolate Ca5_16, which has six promoter repeats. Transformations were performed by Agrobacterium tumefaciens mediated transformation (Díaz-Trujillo et al. unpublished data) using the sensitive wt $P$. fijiensis isolate E22, with a single repeat element and no mutations in the coding region. The promoter length of 250 GFP labelled transformants was compared with the promoter length of the resistant donor Ca5_16 and the sensitive recipient isolate E22. Transformants with a Ca5_16 sized promoter are considered to be homologous recombinants, hence promoter swapped transformants, which were subsequently analysed for the integration site using PCR of a 2,629bp amplicon using primers PROM-HR-3' Fwd (5'-TGAGCATTTGAGAGC-3') and Rev (5'TTATGATCGCCTCCAAGC-3') located in the cassette and the Pfcyp51 ORF, respectively. 


\section{Acknowledgements}

CDT thanks the National Council of Science and Technology (CONACyT) from Mexico for PhD scholarship \# 187781. PC is thankful to the Escuela Superior Politécnica del Litoral (ESPOL) and the National Secretary of Higher Education, Science and Technology from Ecuador (SENESCYT) and Syngenta for a $\mathrm{PhD}$ scholarship. RA is grateful to the Universidad Nacional de Colombia, sede Medellín for funding collaborative research with Wageningen University and Research and Wageningen Plant Research. Amir Mirzadi Gohari is gratefully acknowledged for technical advice on Pseudocercospora fijiensis transformation. This research was partially enabled through a grant of the Dutch Dioraphte Foundation. 


\section{References}

Akins, R.A., Sobel, J.D., 2009. Antifungal targets, mechanisms of action, and resistance in Candida albicans, in: Mayers, D.L. (Ed.), Antimicrobial Drug Resistance. Humana Press, Totowa, NJ, pp. 347-407

Albarrag, A.M., Anderson, M.J., Howard, S.J., Robson, G.D., Warn, P.A., Sanglard, D., Denning, D.W., 2011. Interrogation of related clinical pan-azole-resistant Aspergillus fumigatus strains: G138C, Y431C, and G434C single nucleotide polymorphisms in cyp51A, upregulation of cyp51A, and integration and activation of transposon Atf1 in the cyp51A promoter Antimicrob. Agents Chemother. 55, 5113-5121. doi:10.1128/AAC.00517-11

Alvarez-Rueda, N., Fleury, A., Morio, F., Pagniez, F., Gastinel, L., Le Pape, P., 2011. Amino acid substitutions at the major insertion loop of Candida albicans Sterol 14alpha-demethylase are involved in fluconazole resistance. PLOS ONE 6, e21239. doi:10.1371/journal.pone.0021239

Amil, A.F., Heaney, S.P., Stanger, C., Shaw, M.W., 2007. Dynamics of QoI sensitivity in Mycosphaerella fijiensis in Costa Rica during 2000 to 2003. Phytopathology 97, 1451-1457. doi:10.1094/PHYTO-97-11-1451

Arango, R.E., Díaz-Trujillo, C., Dhillon, B., Aerts, A., Carlier, J., Crane, C.F., Jong, T.V. de, Vries, I. de, Dietrich, R., Farmer, A.D., Fortes Fereira, C., Garcia, S., Guzmán, M., Hamelin, R.C., Lindquist, E.A., Mehrabi, R., Quiros, O., Schmutz, J., Shapiro, H., Reynolds, E., Scalliet, G., Souza Jr, M., Stergiopoulos, I., Van der Lee, T.A.J., Wit, P.J.G.M. de, Zapater, M.-F., Zwiers, L.-H., Grigoriev, I.V., Goodwin, S.B., Kema, G.H.J., 2016. Combating a global threat to a clonal crop: the banana black Sigatoka pathogen Pseudocercospora fijiensis (synonym Mycosphaerella fijiensis) genomes reveals clues for disease control. PLoS Genet 12(8): e1005876. doi: 10.1371/journal.pgen.1005876

Becher, R., Wirsel, S.G.R., 2012. Fungal cytochrome P450 sterol 14 $\alpha$-demethylase (CYP51) and azole resistance in plant and human pathogens. Appl Microbiol Biotechnol 95, 825-840. doi:10.1007/s00253-012-4195-9

Cañas-Gutiérrez, G.P., Angarita-Velásquez, M.J., Restrepo-Flórez, J.M., Rodríguez, P., Moreno, C.X., Arango, R., 2009. Analysis of the CYP51 gene and encoded protein in propiconazole-resistant isolates of Mycosphaerella fijiensis. Pest. Manag. Sci. 65, 892-899. doi:10.1002/ps.1770

Cañas-Gutiérrez, G.P., Patiño L.F., Rodriguez-Arango, E., Arango, R., 2006. Molecular characterization of benomyl-resistant isolates of Mycosphaerella fijiensis collected in Colombia J. -phytopathol 154, 403-409. doi:10.1111/j.1439-0434.2006.01113.x

Carter, H.E., Fraaije, B.A., West, J.S., Kelly, S.L., Mehl, A., Shaw, M.W., Cools, H.J., 2014. Alterations in the predicted regulatory and coding regions of the sterol $14 \alpha$-demethylase gene (CYP51) confer decreased azole sensitivity in the oilseed rape pathogen Pyrenopeziza brassicae. Mol. Plant Pathol. 15, 513-522. doi:10.1111/mpp.12106

Chen, X., Xue, W., Zhou, J., Zhang, Z., Wei, S., Liu, X., Sun, X., Wang, W., Li, S., 2016. Derepression of CSP-1 activates adaptive responses to antifungal azoles. Sci Rep 6, 19447. doi:10.1038/srep19447

Chong-Aguirre, P. 2016. The origin, versatility and distribution of azole fungicide resistance in the banana black Sigatoka pathogen Pseudocercospora fijiensis. PhD Thesis, Wageningen University, The Netherlands. doi:1018174/387237

Conde-Ferráez, L., Waalwijk, C., Canto-Canché, B.B., Kema, G.H.J., Crous, P.W., James, A.C., Abeln, E.C.A., 2007. Isolation and characterization of the mating type locus of Mycosphaerella fijiensis, the causal agent of black leaf streak disease of banana. Mol Plant Pathol. 8, 111-120. doi:10.1111/j.1364-3703.2006.00376.x

Cools, H.J., Bayon, C., Atkins, S., Lucas, J.A., Fraaije, B.A., 2012. Overexpression of the sterol $14 \alpha$-demethylase gene (MgCYP51) in Mycosphaerella graminicola isolates confers a novel azole fungicide sensitivity phenotype. Pest. Manag. Sci. 68, 1034-1040. doi:10.1002/ps.3263 
Cools, H.J., Fraaije, B.A., 2013. Update on mechanisms of azole resistance in Mycosphaerella graminicola and implications for future control. Pest. Manag. Sci. 69, 150-155. doi:10.1002/ps.3348

Crooks, G.E., Hon, G., Chandonia, J.-M., Brenner, S.E., 2004. WebLogo: a sequence logo generator. Genome Res. 14, 1188-1190. doi:10.1101/gr.849004

De Lapeyre De Bellaire, L., Abadie, C., Carlier, J., Ngando, J. and Kema, G.H.J. 2010. Mycosphaerella foliar diseases of bananas: towards an integrated protection. From Science to Field Banana Case Study-Guide 2. ENDURE, Paris (FRA). 8p. http://www.endurenetwork.eu/about_endure/all_the_news/new_guides_for_better_banana_production

Deighton, F.C., 1976. Studies on Cercospora and allied genera. VI. Pseudocercospora Cif. and Cercoseptoria Petr. Mycol. Pap. 140, 1-168

FRAC, 2010. FRAC Recommendations for fungicide mixtures - January 2010.

Frenkel, O., Cadle-Davidson, L., Wilcox, W.F., Milgroom, M.G., 2014. Mechanisms of resistance to an azole fungicide in the grapevine powdery mildew fungus, Erysiphe necator. Phytopathology 105, 370-377. doi:10.1094/PHYTO-07-14-0202-R

Hayashi, K., Schoonbeek, H., De Waard, M.A., 2002. Expression of the ABC transporter BcatrD from Botrytis cinerea reduces sensitivity to sterol demethylation inhibitor fungicides. Pestic Biochem Phys 73, 110-121. doi:10.1016/S0048-3575(02)00015-9

Hayden, H.L., Carlier, J., 2003. Genetic structure of Mycosphaerella fijiensis populations from Australia, Papua New Guinea and the Pacific Islands. Plant Pathol. 52, 703712doi:10.1111/j.1365-3059.2003.00883.x

Lepesheva, G.I., Waterman, M.R., 2011. Structural basis for conservation in the CYP51 family. BBA - Proteins Proteom. Cytochrome P450: Structure, biodiversity and potential for application 1814, 88-93. doi:10.1016/j.bbapap.2010.06.006

Leroux, P., Walker, A.-S., 2011. Multiple mechanisms account for resistance to sterol $14 \alpha-$ demethylation inhibitors in field isolates of Mycosphaerella graminicola. Pest. Manag. Sci. 67, 44-59. doi:10.1002/ps.2028

Li, M., Wang, J., Geng, Y., Li, Y., Wang, Q., Liang, Q., Qi, Q., 2012. A strategy of gene overexpression based on tandem repetitive promoters in Escherichia coli. Microb. Cell Factories 11, 19. doi:10.1186/1475-2859-11-19

Liu, F., Pu, L., Zheng, Q., Zhang, Y., Gao, R., Xu, X., Zhang, S., Lu, L., 2015. Calcium signalling mediates antifungal activity of triazole drugs in the Aspergilli. Fungal Genet Biol. 141, 237246. doi:10.1016/j.fgb.2014.12.005

Livak, K.J., Schmittgen, T.D., 2001. Analysis of relative gene expression data using real-time quantitative PCR and the 2- $\triangle \Delta \mathrm{CT}$ method. Methods 25, 402-408. doi:10.1006/meth.2001.1262

Luo, C.-X., Schnabel, G., 2008. Adaptation to fungicides in Monilinia fructicola isolates with different fungicide resistance phenotypes. Phytopathology 98, 230-238. doi:10.1094/PHYTO98-2-0230

Ma, Z., Proffer, T.J., Jacobs, J.L., Sundin, G.W., 2006. Overexpression of the 14alpha-demethylase target gene (CYP51) mediates fungicide resistance in Blumeriella jaapii. Appl. Environ. Microbiol. 72, 2581-2585. doi:10.1128/AEM.72.4.2581-2585.2006

Mair, W. J., Deng, W., Mullins, J. G. L., West, S., Wang, P., Besharat, N., Ellwood S. R., Oliver, R. P., Lopez-Ruiz F. J. 2016. Demethylase inhibitor fungicide resistance in Pyrenophora teres f. sp. teres associated with target site modification and inducible overexpression of Cyp51. Front Microbiol. 7, 1279-1295. doi:10.3389/fmicb.2016.01279

Marín, D.H., Romero, R.A., Guzmán, M., Sutton, T.B., 2003. Black Sigatoka: An increasing threat to banana cultivation. Plant Dis. 87, 208-222. doi:10.1094/PDIS.2003.87.3.208

Martínez-Bolaños, L., Téliz-Ortiz, D., Rodríguez-Maciel, J.C., Mora-Aguilera, J.A., Nieto-Ángel, D., Cortés-Flores, J.I., Mejía-Sánchez, D., Nava-Diaz, C., Silva-Aguayo, G., 2012. Resistencia a fungicidas en poblaciones de Mycosphaerella fijiensis del sureste mexicano. Agrociencia 46, 707-717.

Matthews, G.A., Baterman, R., Miller, P., 2014. Chemical control in integrated pest management, in: Pesticide Application Methods. John Wiley \& Sons, Oxford. 
Mellado, E., Garcia-Effron, G., Alcázar-Fuoli, L., Melchers, W.J.G., Verweij, P.E., CuencaEstrella, M., Rodríguez-Tudela, J.L., 2007. A new Aspergillus fumigatus resistance mechanism conferring in vitro cross-resistance to azole antifungals involves a combination of cyp51A alterations. Antimicrob. Agents Chemother. 51, 1897-1904. doi:10.1128/AAC.0109206

Montoya, P., Enrique, J., David, V., Estella, L., Brito, D., Judith, T., Sánchez, C., Antonio, D., Rodríguez Beltrán, E., Isaza, A., Eduardo, R., 2006. Use of a micro title plate dilution assay to measure activity of antifungal compounds against Mycosphaerella fijiensis, Morelet. Rev. Fac. Nac. Agron. Medellín 59, 3425-3433

Morelet, M., 1969. Micromycetes du Var et d'ailleurs (2me Note). Annales de la Société des Sciences Naturelles et d'Archeologic de Toulon et du Var 21, 104-106.

Mullins, J.G.L., Parker, J.E., Cools, H.J., Togawa, R.C., Lucas, J.A., Fraaije, B.A., Kelly, D.E., Kelly, S.L., 2011. Molecular modelling of the emergence of azole resistance in Mycosphaerella graminicola. PLoS ONE 6, e20973. doi:10.1371/journal.pone.0020973

Nikou, D., Malandrakis, A., Konstantakaki, M., Vontas, J., Markoglou, A., Ziogas, B., 2009. Molecular characterization and detection of overexpressed C-14 alpha-demethylase-based DMI resistance in Cercospora beticola field isolates. Pestic Biochem Phys 95, 18-27. doi:10.1016/j.pestbp.2009.04.014

Price, C.L., Parker, J.E., Warrilow, A.G., Kelly, D.E., Kelly, S.L., 2015. Azole fungicides understanding resistance mechanisms in agricultural fungal pathogens. Pest Manag. Sci. 71, 1054-1058. doi:10.1002/ps.4029

Rallos, L.E.E., Baudoin, A.B., 2016. Co-occurrence of two allelic variants of CYP51 in Erysiphe necator and their correlation with over-expression for DMI resistance. PLoS ONE 11, e0148025. doi:10.1371/journal.pone.014802

Rivas, G.-G., Zapater, M.-F., Abadie, C., Carlier, J., 2004. Founder effects and stochastic dispersal at the continental scale of the fungal pathogen of bananas Mycosphaerella fijiensis. Mol.Ecol. 13, 471-482. doi:10.1046/j.1365-294X.2003.02043.x

Romero, R.A., Sutton, T.B., 1997. Sensitivity of Mycosphaerella fijiensis, causal agent of black Sigatoka of banana, to propiconazole. Phytopathology 87, 96-100. doi:10.1094/PHYTO.1997.87.1.96

Scalliet, G., Bowler, J., Luksch, T., Kirchhofer-Allan, L., Steinhauer, D., Ward, K., Niklaus, M., Verras, A., Csukai, M., Daina, A., Fonné-Pfister, R., 2012. Mutagenesis and functional studies with succinate dehydrogenase inhibitors in the wheat pathogen Mycosphaerella graminicola. PLoS ONE 7, e35429. doi:10.1371/journal.pone.0035429

Schnabel, G., Jones, A.L., 2001. The 14a-demethylasse(CYP51A1) gene is overexpressed in Venturia inaequalis strains resistant to myclobutanil. Phytopathology 91, 102-110. doi:10.1094/PHYTO.2001.91.1.102

Snelders, E., Camps, S. M. T., Karawajczyk, A., Schaftenaar, G., Kema, G. H. J., van der Lee, H. A., Klaassen, C.H., Melchers, W.J.G., Verweij, P. E. 2012. Triazole fungicides can induce cross-resistance to medical triazoles in Aspergillus fumigatus. PLoS ONE, 7(3), e31801. doi: 10.1371/journal.pone.0031801

Sun, X., Wang, K., Yu, X., Liu, J., Zhang, H., Zhou, F., Xie, B., Li, S., 2014. Transcription factor CCG-8 as a new regulator in the adaptation to antifungal azole stress. Antimicrob Agents Chemother. 58, 1434-1442. doi:10.1128/AAC.02244-13

Sun, X., Xu, Q., Ruan, R., Zhang, T., Zhu, C., Li, H., 2013. PdMLE1, a specific and active transposon acts as a promoter and confers Penicillium digitatum with DMI resistance. Environ. Microbiol. Rep. 5, 135-142. doi:10.1111/1758-2229.12012

Verweij, P.E., Kema, G.H., Zwaan, B., Melchers, W.J., 2013. Triazole fungicides and the selection of resistance to medical triazoles in the opportunistic mould Aspergillus fumigatus. Pest. Manag. Sci. 69, 165-170. doi:10.1002/ps.3390

Verweij PE, Mellado E, Melchers WJ., 2007. Multiple-triazole-resistant aspergillosis. $N$ Engl J Med 356: 1481-1483. doi: 10.1056/NEJMc061720 
Villani, S.M., Hulvey, J., Hily, J.-M., Cox, K.D., 2016. Overexpression of the CYP51A1 gene and repeated elements are associated with differential sensitivity to DMI fungicides in Venturia inaequalis. Phytopathology 106, 562-571. doi:10.1094/PHYTO-10-15-0254-R 
A

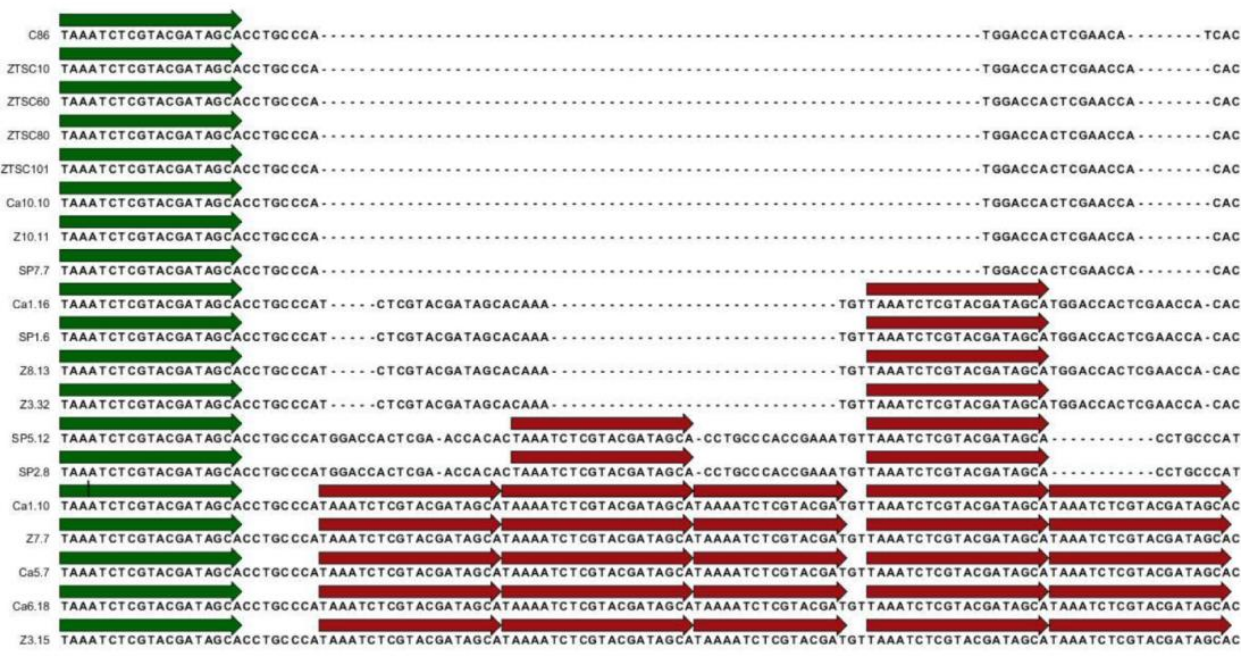

B

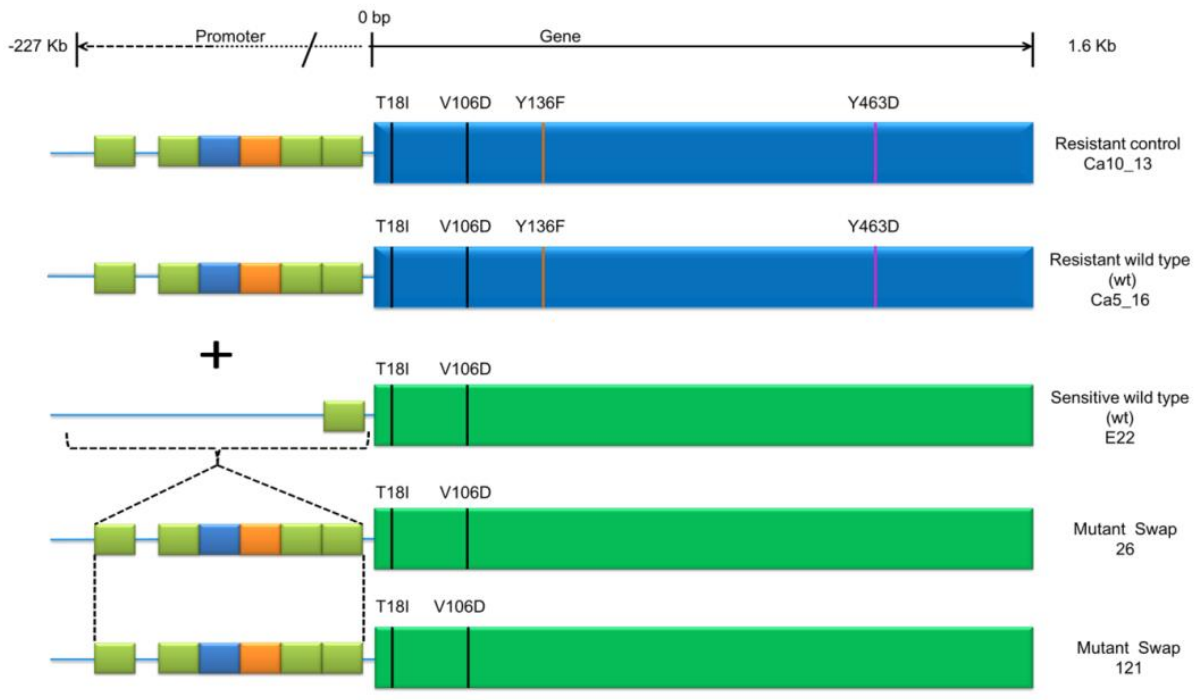

Figure 1. The Pfcyp51 structure. A) Alignment of the promoter regions of the Pfcyp51 gene of Pseudocercospora fijiensis isolates collected from the Zent $(\mathrm{Z})$, Cartagena $(\mathrm{Ca})$, San Pablo (SP) and the wt San Carlos (ZTSC) banana plantations in Costa Rica, isolate CIRAD86 (C86) is the reference wt isolate, the repeat element present in all isolate at position -122 bp is shown in green arrows and additional repeated elements identified in various $P$. fijiensis isolates are shown as red arrows (see for origin of isolates Table 1). B) Configuration of the Pfcyp51 promotor and coding domains of the wt Pseudocercospora fijiensis isolates used to generate transformants. The promoter region is shown at the left as a blue line with different coloured boxes: green, blue and orange boxes represent the $19 \mathrm{bp}, 20 \mathrm{bp}$, or $16 \mathrm{bp}$ promoter repeat elements; rectangular boxes at the right represent the coding regions of the Pfcyp51 gene in these isolates: green represent the sensitive wt and blue the resistant donor (resistant wt) coding region. Vertical lines in the coding regions represent amino acid substitutions. 


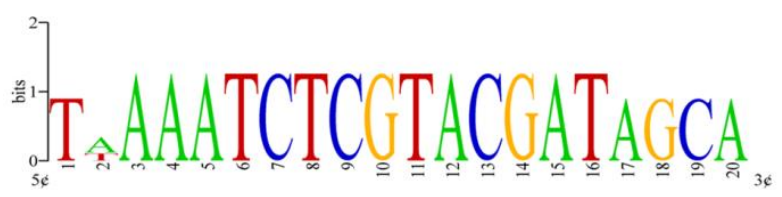

Figure 2. Sequence logo of the Pfcyp51 promoter repeat element. Sequences of all repeat elements were aligned and used to generate the consensus sequence. The logo displays the frequency of the nucleotides within the repeated elements of 16,19 or 20 bp that were observed in the promoter of Pfcyp51 


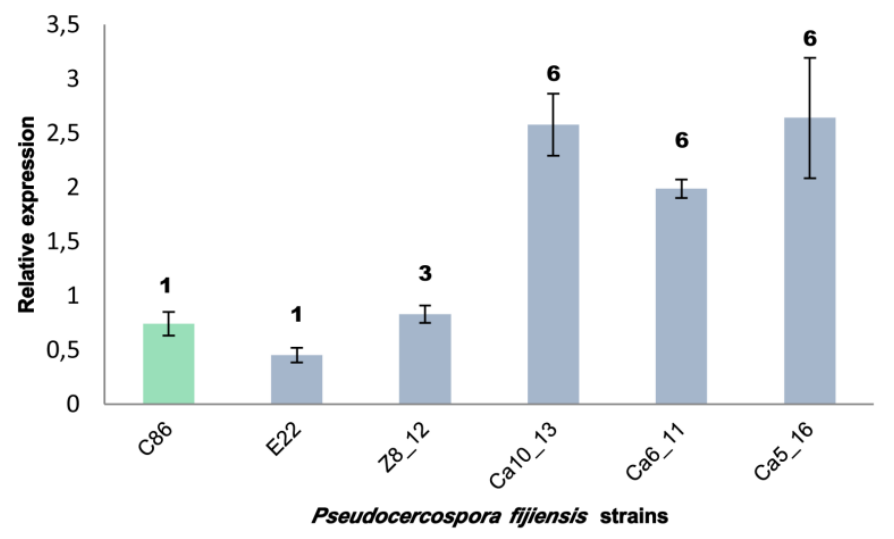

Figure 3. Relative expression of Pfcyp51 (normalized to the Pseudocercospora fijiensis actin gene) in six $P$. fijiensis isolates carrying different numbers of promoter inserts (indicated on the top of each bar). Reference isolate CIRAD86 (C86) is shown in green. Data represent the averages of three biological repetitions each with at least three technical replicates (error bars indicate standard deviations). 
A

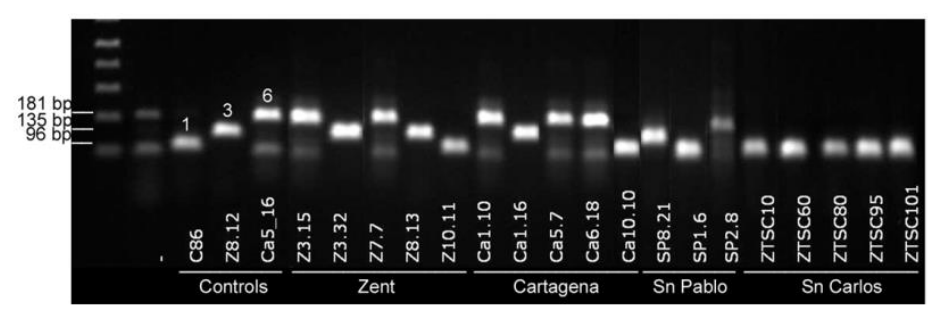

B

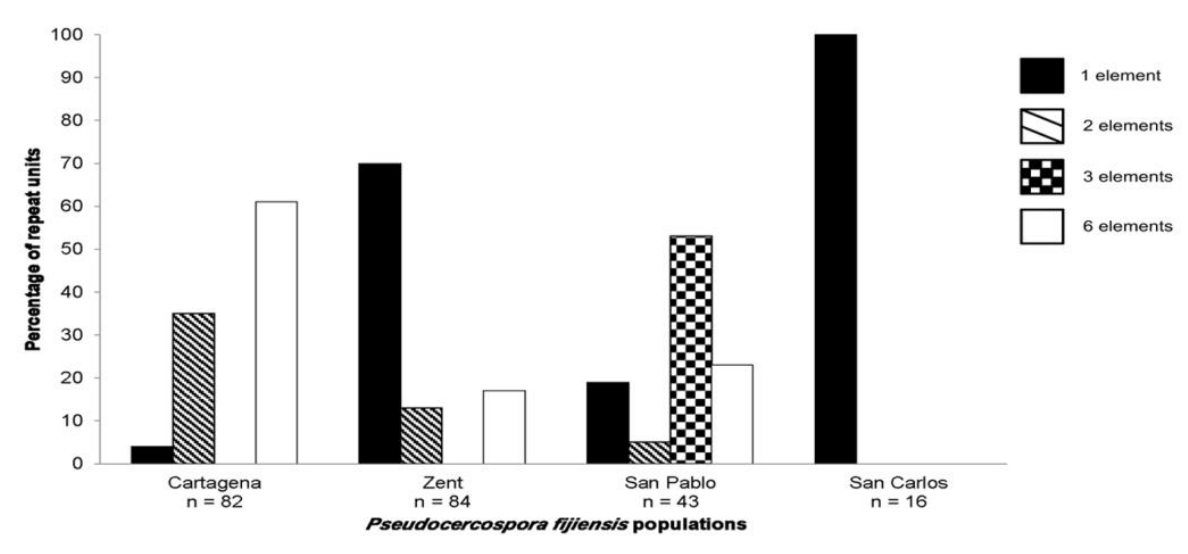

Figure 4. Quantification of the number of Pfcyp51 promoter repeats in Pseudocercospora fijiensis isolates from four banana plantations in Costa Rica. (A) Example of polymerase chain reaction (PCR) amplification of the Pfcyp51 promoter in isolates from different populations. Isolate CIRAD86 (C86) was used as a control for the presence of one repeat element, Z8.12 as a control with three repeat elements and Ca5_16 as a control with six repeat elements. The number of repeat elements in each control sample is indicated above the corresponding amplicon. The other isolates originated from banana plantations extensively treated (or not) with azole fungicides and contain varying numbers of repeat elements in the Pfcyp51 promoter. (B) Distribution of repeat elements in the Pfcyp51 promoter within Costa Rican populations of $P$. fijiensis, based on 225 PCR amplifications. 

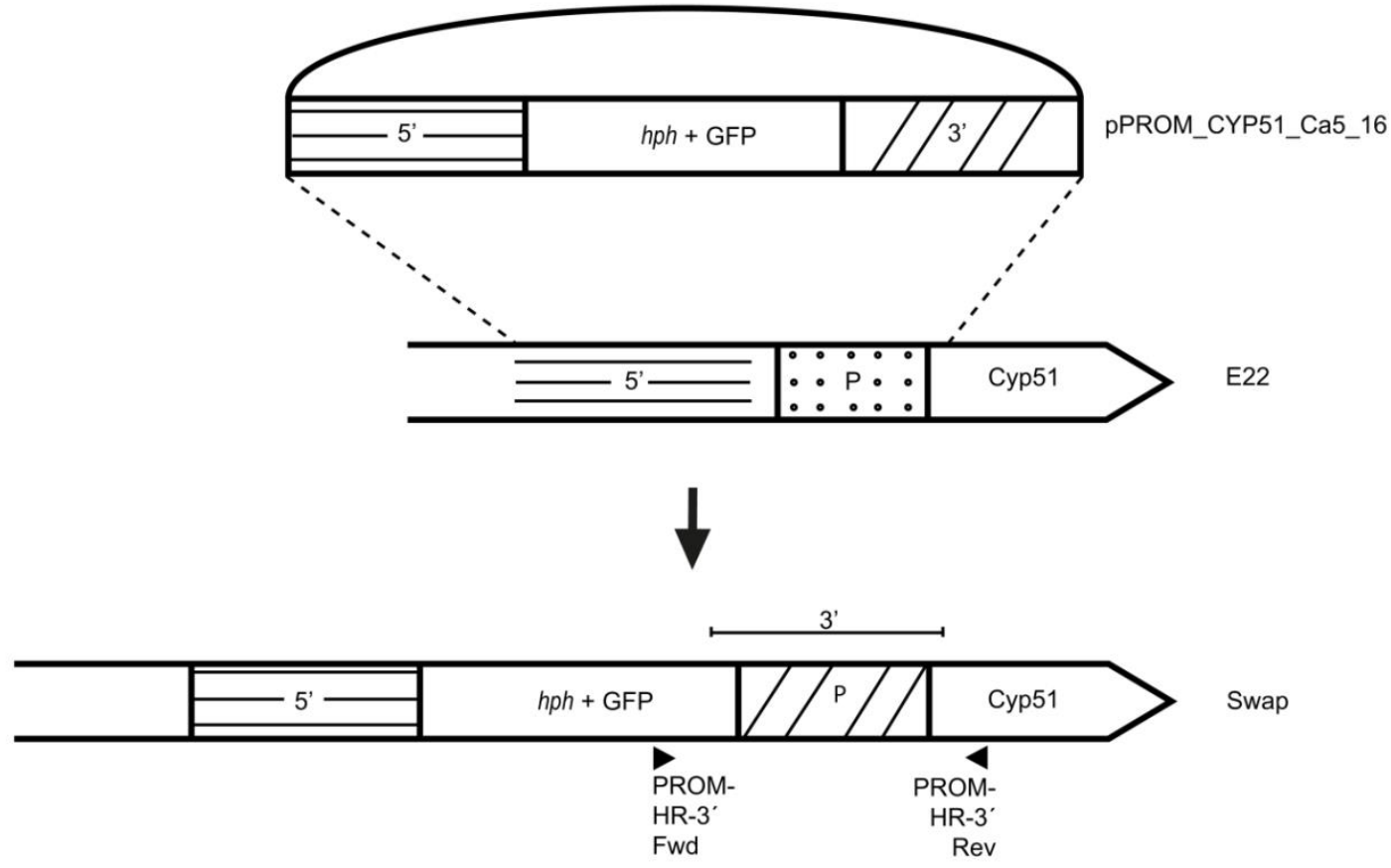

b

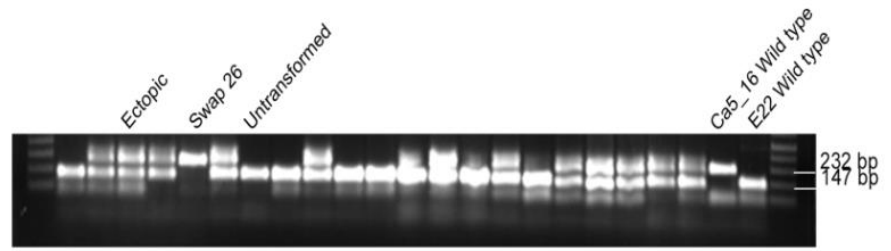

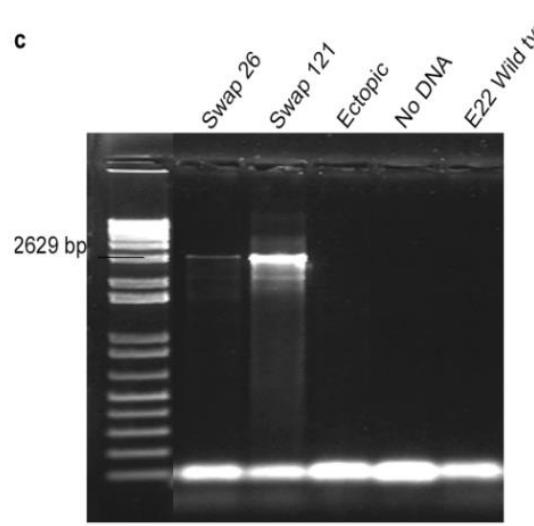

Figure 5. Transformation design to swap Pfcyp51 promoters of Pseudocercospora fijiensis isolates. (A) Isolate Ca5_16 is the Pfcyp51 promoter donor with six repeat elements (slashed area part with the cross lines). The 3'and 5'recombination fragments (crossed out area part with the horizontal lines) were amplified with CYP-Prom primers and ligated to a cassette with the hph and green fluorescent protein (GFP) markers into construct pPROM_CYP51_Ca5_16. The P. fijiensis E22 sensitive isolate with one 19-bp promoter element (dotted area) was transformed with this construct. (B) The promoter lengths of positive GFP-tagged transformants were amplified and compared with the donor and wildtype (wt) recipient isolate. Transformant Swap 26 is shown as an example of a promoter replacement transformant, with a similar amplicon to the donor isolate. Ectopic transformats possess the promoter fragment of both the donor and the recipient isolate, whereas untransformed isolates only show the wt-sized amplicon. (C) Detection and characterization of promoter swapped transformants were performed by amplification of the 2629-bp cassette between the homologous recombination sites and the Pfcyp51 coding region using primers PROM-HR-3'on GFP fluorescent transformants with a promoter amplicon similar to the donor isolate. 
A

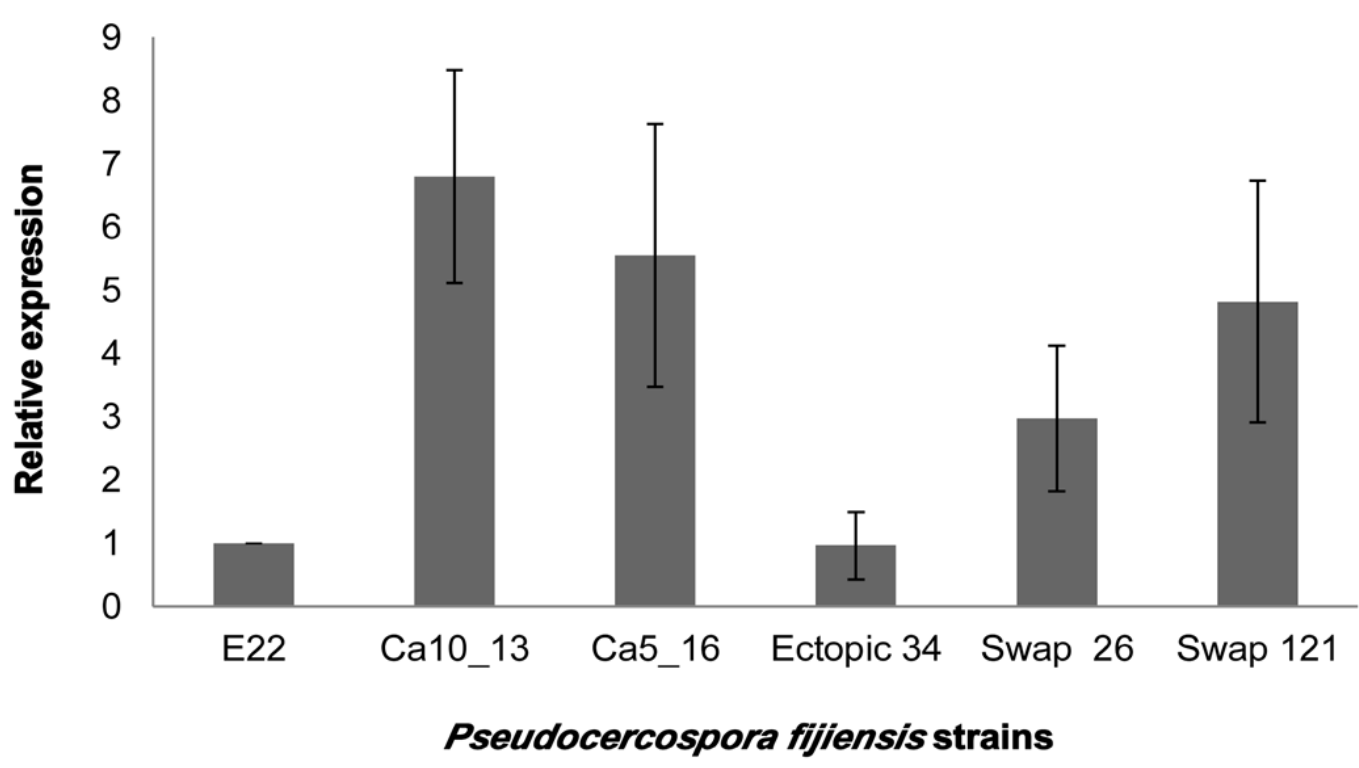

B

\begin{tabular}{llll}
\hline Sample & Difenoconazole & Epoxiconazole & Propiconazole \\
\hline Ca10_13 (Resistant) & $5,629 \pm 0,1789$ & $4,646 \pm 0,1818$ & $5,653 \pm 0,1905$ \\
E22 (Sensitive) & $0,008 \pm 0,0009$ & $0,025 \pm 0,0014$ & $0,026 \pm 0,0012$ \\
Swap 26 & $0,016 \pm 0,0062$ & $0,112 \pm 0,0205$ & $0,121 \pm 0,0228$ \\
Swap 121 & $0,034 \pm 0,0010$ & $0,209 \pm 0,0450$ & $0,136 \pm 0,0370$ \\
Ectopic & $0,003 \pm 0,0001$ & $0,023 \pm 0,0011$ & $0,014 \pm 0,0016$ \\
\hline
\end{tabular}

Figure 6. In vitro sensitivity of Pseudocercospora fijiensis transformants Swap26 and Swap121 with swapped Pfcyp51 promoters vs. various control isolates. (A) The relative expression (normalized with the expression in wt sensitive donor isolate E_22) of Pfcyp51 in Swap26 and Swap121, the wt E22 and the resistant isolate (Ca10_13) with identical promoter and coding region as donor isolate $\left(\mathrm{Ca} 5 \_16\right)$ as well as the ectopic control isolate (Ectopic 34). Data represent the averages of three replications. (B) Table with means of $\mathrm{EC}_{50}$ values $\left(\mathrm{mg} \cdot \mathrm{L}^{-1}\right)$ of the Pseudocercospora fijiensis promotor swapped transformants Swap26 and Swap121 and various control isolates to three azole fungicides. 
Table 1. Origin and characteristics of the Pfcyp51 gene and its promoter in 25 Pseudocercospora fijiensis isolates used in this study, including their sensitivity to propiconazole and cyproconazole $\left(\mathrm{EC}_{50}\right)$.

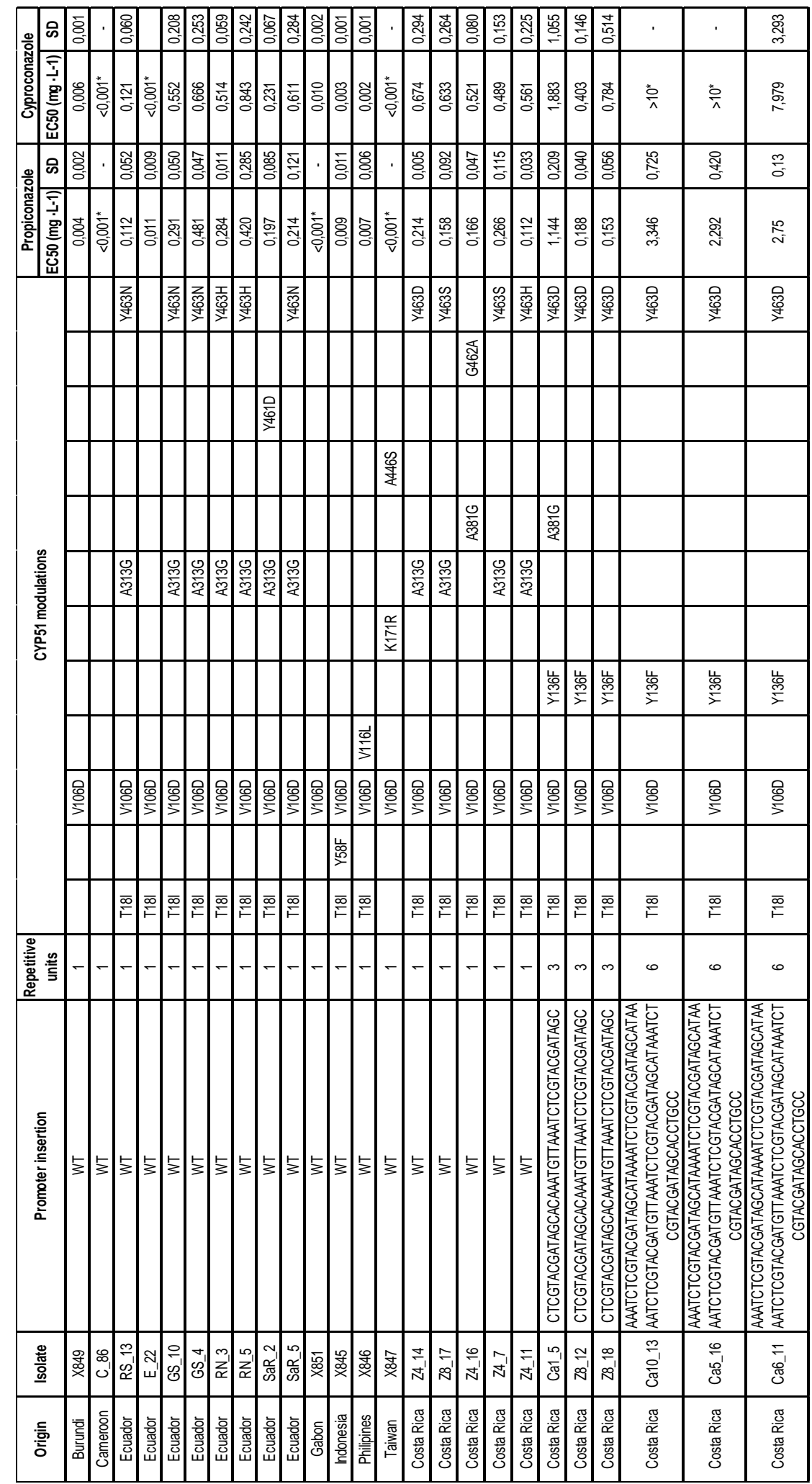

*Out of dose range for calculations

$\mathrm{SD}=$ Standard deviation 


\title{
Supplementary data
}

Text S1 Genomic sequence of Pfcyp51 in a set of 25 isolates of Pseudocercospora fijiensis from Asia, Africa and Latin America.

\begin{abstract}
$>\mathrm{C} 86$
ATGCGGCGTGATGGTTGGACCCTTGGACCCTCGCAATTGATGAGAAGCAGGGGTGTCCCGTTCCTGCATGACTAGCGCC AAAAAGTGACGCGACTGCCGAAATGTTAAATCTCGTACGATAGCACCTGCCCATGGACCACTCGAACATCACTGAAGGG TAATCATTCAAGATCTTGGTGATTTGGCCTAACCCCCTACACCAACATCAACATCACTGACTCCGCGCAATGGGGCTCC TCCAGGACGCCGCGGCGCTTTTCGACGCGCAATTTGGCCAGACAGCGACATGGAAACTAGTCCCCCTCGGCTTCAGCAT CTTCTTCGCCGTATCCGTGTTGCTTAACGTGTTGCGCCAGCTGCTCTTCAGAAATCCAAACGAACCTCCGCTAGTATTC CACTACGTGCCCTTCATTGGCAGCACTATCTCCTATGGCATCGACCCCTACAAGTTCTTCTTCGCCTGCCGTCAAAAAT ACGGAGATTGCTTCACTTTCATCCTCCTTGGCAAGAAGACCACCGTGGTGCTGGGGACTAAAGGCAACGTCTTTATCTT GAATGGAAAGCTCAAGGACGTCAATGCCGAGGAGATCTATAGCCCACTTACTACGCCAGTATTCGGCACAGATGTCGTC TACGATTGTCCCAATTCGAAGCTCATGGAGCAGAAGAAGGTGCGTGCACACAATAAGCTGGATTTATGACAATGCTAAC TTATACAGTTCGTCAAATATGGTCAGTGACTTGACTCCGCCGCGTCTGAGACAGCGCTCTCAACATGAGCTTCCACTTT GGAGCGATACACTTGCTAACCTGCGCCTCCAGGCCTCACCTCCTCCGCCCTCCAGTCCTACGTTAAATTGATCACCAAA GAGACCAAAGACTTCTTCTCCAAGGACAATCCAAGCAAGAAATTCGCATCCACCCACGGCACCGTCGACCTCCCGCCTG CTATGGCTGAGCTTACTATCTACACCGCCAGCCGTTCGCTCCAGGGCAAAGAAGTCCGCGAAAAATTCGACTCCTCCTT TGCCGACCTCTACCACGATCTCGACATGGGCTTCACTCCCATCAACTTCATGCTTCCATGGGCTCCACTGCCACAGAAT AGAGCACGCGATCGCGCACAGAAGAAGATGGCGGAAGTCTACACAGCGATCATCAAAGAGCGACGCGAAAAGGGCGAGC CTACTTCGGGAGAGAAAGAGCAGGACATGATTTGGAATCTGATGCAATGTCAGTACAAGAATGGTCAAGCAATTCCAGA TAAGGAGATTGCGCACATGATGATTGCCCTTCTCATGGCTGGTCAACACTCGTCCTCGTCCACCTCATGCTGGATCCTT CTCCGACTGGCTTCGCGACCAGATATCCAGGATGAGCTCCTTCAAGAACAGAAGGATGTGCTTGGCGTCAACGCAGATG GATCAATCAAGGAGTTAACGTACGCCGACATCTCGCGCCTTCCACTCCTCAATCAAGTTGTCAAGGAGACACTCCGCCT TCATGCTCCCATCCATTCTATTCTGCGACAAGTCAAGTCTCCGATGCCACTCGAAGGTACACCATACGTTGTCCCGACC ACACACTCCCTCCTTGCTGCACCCGGTGCTACCTCACGAATGGACGAGCACTTCCCCGAAGCTATGCTGTGGGAACCCC ACCGATGGGACGAGAACCCAAGTGAGAAGTACGCACATCTCGCACCAAAGCATGTCAAGGAGGGCGTCGCCGAAGAGAC TGAAGATTACGGCTATGGTCTCGTCAGCAAAGGCGCCGCATCACCATATCTGCCATTCGGTGCTGGCCGACATAGATGC ATCGGCGAGCAATTCGCCTATGTCCAGCTCCAGACCATCACCTCGGAAGTGATTCGCGATTTCAAGCTCTACAATGTCG ACGGCAGCGACAAAGTTGTCGGCACAGATTACAGTTCGTTGTTCAGCAGACCTCTCTCGCCAGCCGTCGTGCGATGGGA GAGGAGAGAAAAGAAATAGATTTCACAGTAAGAGTATGTTAATGCTAATCAGACAACTATATCCATATCTGCAGCTTCC CTCCTCTGCGAGA
\end{abstract}

\begin{abstract}
$>$ Z4_7_Consensus
TGCAGATGATGGTTGGACCCTTGCGACCCTCGCAATTGA

TGAGAAGCAGGGGTGTCCCGTTCCTGCATGACTAGCGCCAAAAAGTGACGCGAATGCCGA

AATGTTAAATCTCGTACGATAGCACCTGCCCATGGACCAC

TCGAACCACACTGAAGGGTAATCATTCAAGATCTTGGTGATTTGGCCTAACCCCC

TACACCAACATCAACATCACTGACTCCGCGCAATGGGACTCCTCCAGGACGCCGCGGCGC

TTTTCGACGCGCAATTTGGCCAGATAGCGACATGGAAACTAGTCCCCCTCGGCTTCAGCA TCTTCTTCGCCGTATCCGTGTTGCTTAACGTGTTGCGCCAGCTGCTTTTCAGAAATCCAA ACGAACCTCCGCTAGTATTCCACTACGTGCCCTTCATTGGCAGCACTATCTCCTATGGCA TCGACCCCTACAAGTTCTTCTTCGCCTGCCGTCAAAAATACGGAGATTGCTTCACTTTCA TCCTCCTTGGCAAGAAGACCACCGTGGTGCTGGGGACTAAAGGCAACGACTTTATCTTGA ATGGAAAGCTCAAGGACGTCAATGCCGAGGAGATCTATAGCCCACTTACTACGCCAGTAT TCGGCACAGATGTCGTCTACGATTGTCCCAATTCGAAGCTCATGGAGCAGAAGAAGGTGC GTGCACACAATAAGCTGGATTTATGACAATGCTAACTTATACAGTTCGTCAAATATGGTC AGTGACTTGACTCCGCCGCGTCTGAGACAGCGCTCTCAACATGAGCTTCCACTTTGGAGC GATACACTTGCTAACCTGCGCCTCCAGGCCTCACCTCCTCCGCCCTCCAGTCCTACGTTA AATTGATCACCAAAGAGACCAAAGACTTCTTCTCCAAGGACAATCCAAGCAAGAAATTCG CATCCACCCACGGCACCGTCGACCTCCCGCCTGCTATGGCTGAGCTTACTATCTACACCG CCAGCCGTTCGCTCCAGGGCAAAGAAGTCCGCGAAAAATTCGACTCCTCCTTTGCCGACC TCTACCACGATCTCGACATGGGCTTCACTCCCATCAACTTCATGCTTCCATGGGCTCCAC TGCCACAGAATAGAGCACGCGATCGCGCACAGAAGAAGATGGCGGAAGTCTACACAGCG ATCATCAAAGAGCGACGCGAAAAGGGCGAGCCTACTTCGGGAGAGAAAGAGCAGGACATG ATTTGGAATCTGATGCAATGTCAGTACAAGAATGGTCAAGCAATTCCAGATAAGGAGATT GCGCACATGATGATTGCCCTTCTCATGGGTGGTCAACACTCGTCCTCGTCCACCTCATGC TGGATCCTTCTCCGACTGGCTTCGCGACCAGATATCCAGGATGAGCTCCTTCAAGAACAG AAGGATGTGCTTGGCGTCAACGCAGATGGATCAATCAAGGAGTTAACGTACGCCGACATC TCGCGCCTTCCACTCCTCAATCAAGTTGTCAAGGAGACACTCCGCCTTCATGCTCCCATC CATTCTATTCTGCGACAAGTCAAGTCTCCGATGCCACTCGAAGGTACACCATACG TTGTC CCGACCACACACTCCCTCCTTGCTGCACCCGGTGCTACCTCACGAATGGACGAGCACTTC CCCGAAGCTATGCTGTGGGAACCCCACCGATGGGACGAGAACCCAAGTGAGAAGTACGCA
\end{abstract}


CATCTCGCACCAAAGCATGTCAAGGAGGGCGTCGCCGAAGAGACTGAAGATTACGGCTCT GGTCTCGTCAGCAAAGGCGCCGCATCACCATATCTGCCATTCGGTGCTGGCCGACATAGA TGCATCGGCGAGCAATTCGCCTATGTCCAGCTCCAGACCATCACCTCGGAAGTGATTCGC GATTTCAAGCTCTACAATGTCGACGGCAGCGACAAAGTTGTCGGCACAGATTACAGTTCG TTGTTCAGCAGACCTCTCTCGCCAGCCGTCGTGCGATGGGAGAGGAGAGAAAGAAATA GATTTCACAGTA

\begin{abstract}
$>$ Z4_11_Consensus GGTGCAGATGATGGTTGGACCCTTGGCACCCTCGCAATTGA TGAGAAGCAGGGGTGTCCCGTTCCTGCATGACTAGCGCCAAAAAGTGACGCGAATGCCGA AATGTTAAATCTCGTACGATAGCACCTGCCCATGGACCAC TCGAACCACACTGAAGGGTAATCATTCAAGATCTTGGTGATTTGGCCTAACCCCC TACACCAACATCAACATCACTGACTCCGCGCAATGGGACTCCTCCAGGACGCCGCGGCGC TTTTCGACGCGCAATTTGGCCAGATAGCGACATGGAAACTAGTCCCCCTCGGCTTCAGCA TCTTCTTCGCCGTATCCGTGTTGCTTAACGTGTTGCGCCAGCTGCTTTTCAGAAATCCAA ACGAACCTCCGCTAGTATTCCACTACGTGCCCTTCATTGGCAGCACTATCTCCTATGGCA TCGACCCCTACAAGTTCTTCTTCGCCTGCCGTCAAAAATACGGAGATTGCTTCACTTTCA TCCTCCTTGGCAAGAAGACCACCGTGGTGCTGGGGACTAAAGGCAACGACTTTATCTTGA ATGGAAAGCTCAAGGACGTCAATGCCGAGGAGATCTATAGCCCACTTACTACGCCAGTAT TCGGCACAGATGTCGTCTACGATTGTCCCAATTCGAAGCTCATGGAGCAGAAGAAGGTGC GTGCACACAATAAGCTGGATTTATGACAATGCTAACTTATACAGTTCGTCAAATATGGTC AGTGACTTGACTCCGCCGCGTCTGAGACAGCGCTCTCAACATGAGCTTCCACTTTGGAGC GATACACTTGCTAACCTGCGCCTCCNGGCCTCACCTCCTCCGCCCTCCAGTCCTACGTTA AATTGATCACCAAAGAGACCAAAGACTTCTTCTCCAAGGACAATCCAAGCAAGAAATTCG CATCCACCCACGGCACCGTCGACCTCCCGCCTGCTATGGCTGAGCTTACTATCTACACCG CCAGCCGTTCGCTCCAGGGCAAAGAATTCCGCGAAAAATTCGACTCCTCCTTTGCCGACC TCTACCACGATCTCGACATGGGCTTCACTCCCATCAACTTCATGCTTCCATGGGCTCCAC TGCCACAGAAATAGAGCACGCGATCGCGCACAGAAGA-GATGGCGGAAGTCTACACAGCG ATCATCAAAGAGCGACGCGAAAAGGGCGAGCCTACTTCGGGAGAGAAAGAGCAGGACATG ATTTGGAATCTGATGCAATGTCAGTACAAGAATGGTCAAGCAATTCCAGATAAGGAGATT GCGCACATGATGATTGCCCTTCTCATGGGTGGTCAACACTCGTCCTCGTCCACCTCATGC TGGATCCTTCTCCGACTGGCTTCGCGACCAGATATCCAGGATGAGCTCCTTCAAGAACAG AAGGATGTGCTTGGCGTCAACGCAGATGGATCAATCAAGGAGTTAACGTACGCCGACATC TCGCGCCTTCCACTCCTCAATCAAGTTGTCAAGGAGACACTCCGCCTTCATGCTCCCATC CATTCTATTCTGCGACAAGTCAAGTCTCCGATGCCACTCGAAGGTACACCATACGTTGTC CCGACCACACACTCCCTCCTTGCTGCACCCGGTGCTACCTCACGAATGGACGAGCACTTC CCCGAAGCTATGCTGTGGGAACCCCACCGATGGGACGAGAACCCAAGTGAGAAGTACGCA CATCTCGCACCAAAGCATGTCAAGGAGGGCGTCGCCGAAGAGACTGAAGATTACGGCCAT GGTCTCGTCAGCAAAGGCGCCGCATCACCATATCTGCCATTCGGTGCTGGCCGACATAGA TGCATCGGCGAGCAATTCGCCTATGTCCAGCTCCAGACCATCACCTCGGAAGTGATTCGC GATTTCAAGCTCTACAATGTCGACGGCAGCGACAAAGTTGTCGGCACAGATTACAGTTCG TTGTTCAGCAGACCTCTCTCGCCAGCCGTCGTGCGATGGGAGAGGAGAGAAAAGAAATA GATTTCACAGTAA
\end{abstract}

\title{
>Z4_14_Consensus
}

CGAGATGGTGCAGATGATGGTTGGACCCTTGGACCCTCGCAATTGA

TGAGAAGCAGGGGTGTCCCGTTCCTGCATGACTAGCGCCAAAAAGTGACGCGAATGCCGA AATGTTAAATCTCGTACGATAGCACCTGCCCATGGACCAC

TCGAACCACACTGAAGGGTAATCATTCAAGATCTTGGTGATTTGGCCTAACCCCC TACACCAACATCAACATCACTGACTCCGCGCAATGGGACTCCTCCAGGACGCCGCGGCGC TTTTCGACGCGCAATTTGGCCAGATAGCGACATGGAAACTAGTCCCCCTCGGCTTCAGCA TCTTCTTCGCCGTATCCGTGTTGCTTAACGTGTTGCGCCAGCTGCTTTTCAGAAATCCAA ACGAACCTCCGCTAGTATTCCACTACGTGCCCTTCATTGGCAGCACTATCTCCTATGGCA TCGACCCCTACAAGTTCTTCTTCGCCTGCCGTCAAAAATACGGAGATTGCTTCACTTTCA TCCTCCTTGGCAAGAAGACCACCGTGGTGCTGGGGACTAAAGGCAACGACTTTATCTTGA ATGGAAAGCTCAAGGACGTCAATGCCGAGGAGATCTATAGCCCACTTACTACGCCAGTAT TCGGCACAGATGTCGTCTACGATTGTCCCAATTCGAAGCTCATGGAGCAGAAGAAGGTGC GTGCACACAATAAGCTGGATTTATGACAATGCTAACTTATACAGTTCGTCAAATATGGTC AGTGACTTGACTCCGCCGCGTCTGAGACAGCGCTCTCAACATGAGCTTCCACTTTGGAGC GATACACTTGCTAACCTGCGCCTCCAGGCCTCACCTCCTCCGCCCTCCAGTCCTACGTTA AATTGATCACCAAAGAGACCAAAGACTTCTTCTCCAAGGACAATCCAAGCAAGAAATTCG CATCCACCCACGGCACCGTCGACCTCCCGCCTGCTATGGCTGAGCTTACTATCTACACCG CCAGCCGTTCGCTCCAGGGCAAAGAAGTCCGCGAAAAATTCGACTCCTCCTTTGCCGACC TCTACCACGATCTCGACATGGGCTTCACTCCCATCAACTTCATGCTTCCATGGGCTCCAC TGCCACAGAATAGAGCACGCGATCGCGCACAGAAGAAGATGGCGGAAGTCTACACAGCG ATCATCAAAGAGCGACGCGAAAAGGGCGAGCCTACTTCGGGAGAGAAAGAGCAGGACATG ATTTGGAATCTGATGCAATGTCAGTACAAGAATGGTCAAGCAATTCCAGATAAGGAGATT GCGCACATGATGATTGCCCTTCTCATGGGTGGTCAACACTCGTCCTCGTCCACCTCATGC TGGATCCTTCTCCGACTGGCTTCGCGACCAGATATCCAGGATGAGCTCCTTCAAGAACAG 
AAGGATGTGCTTGGCGTCAACGCAGATGGATCAATCAAGGAGTTAACGTACGCCGACATC TCGCGCCTTCCACTCCTCAATCAAGTTGTCAAGGAGACACTCCGCCTTCATGCTCCCATC CATTCTATTCTGCGACAAGTCAAGTCTCCGATGCCACTCGAAGGTACACCATACGTTGTC CCGACCACACACTCCCTCCTTGCTGCACCCGGTGCTACCTCACGAATGGACGAGCACTTC CCCGAAGCTATGCTGTGGGAACCCCACCGATGGGACGAGAACCCAAGTGAGAAGTACGCA CATCTCGCACCAAAGCATGTCAAGGAGGGCGTCGCCGAAGAGACTGAAGATTACGGCGAT GGTCTCGTCAGCAAAGGCGCCGCATCACCATATCTGCCATTCGGTGCTGGCCGACATAGA TGCATCGGCGAGCAATTCGCCTATGTCCAGCTCCAGACCATCACCTCGGAAGTGATTCGC GATTTCAAGCTCTACAATGTCGACGGCAGCGACAAAGTTGTCGGCACAGATTACAGTTCG TTGTTCAGCAGACCTCTCTCGCCAGCCGTCGTGCGATGGGAGAGGAGAGAAAGAAATA GATTTCACAGTA

\author{
$>$ Z4_16_Consensus \\ CGAGATGGTGCAGATGATGGTTGGACCCTTGG-ACCCTCGCAATTGA
}

TGAGAAGCAGGGGTGTCCCGTTCCTGCATGACTAGCGCCAAAAAGTGACGCGAATGCCGA AATGTTAAATCTCGTACGATAGCACCTGCCCATGGACCAC

TCGAACCACACTGAAGGGTAATCATTCAAGATCTTGGTGATTTGGCCTAACCCCC

TACACCAACATCAACATCACTGACTCCGCGCAATGGGACTCCTCCAGGACGCCGCGGCGC TTTTCGACGCGCAATTTGGCCAGATAGCGACATGGAAACTAGTCCCCCTCGGCTTCAGCA TCTTCTTCGCCGTATCCGTGTTGCTTAACGTGTTGCGCCAGCTGCTTTTCAGAAATCCAA ACGAACCTCCGCTAGTATTCCACTACGTGCCCTTCATTGGCAGCACTATCTCCTATGGCA TCGACCCCTACAAGTTCTTCTTCGCCTGCCGTCAAAAATACGGAGATTGCTTCACTTTCA TCCTCCTTGGCAAGAAGACCACCGTGGTGCTGGGGACTAAAGGCAACGACTTTATCTTGA ATGGAAAGCTCAAGGACGTCAATGCCGAGGAGATCTATAGCCCACTTACTACGCCAGTAT TCGGCACAGATGTCGTCTACGATTGTCCCAATTCGAAGCTCATGGAGCAGAAGAAGGTGC GTGCACACAATAAGCTGGATTTATGACAATGCTAACTTATACAGTTCGTCAAATATGGTC AGTGACTTGACTCCGCCGCGTCTGAGACAGCGCTCTCAACATGAGCTTCCACTTTGGAGC GATACACTTGCTAACCTGCGCCTCCAGGCCTCACCTCCTCCGCCCTCCAGTCCTACGTTA AATTGATCACCAAAGAGACCAAAGACTTCTTCTCCAAGGACAATCCAAGCAAGAAATTCG CATCCACCCACGGCACCGTCGACCTCCCGCCTGCTATGGCTGAGCTTACTATCTACACCG CCAGCCGTTCGCTCCAGGGCAAAGAAGTCCGCGAAAAATTCGACTCCTCCTTTGCCGACC TCTACCACGATCTCGACATGGGCTTCACTCCCATCAACTTCATGCTTCCATGGGCTCCAC TGCCACAGAATAGAGCACGCGATCGCGCACAGAAGAAGATGGCGGAAGTCTACACAGCG ATCATCAAAGAGCGACGCGAAAAGGGCGAGCCTACTTCGGGAGAGAAAGAGCAGGACATG ATTTGGAATCTGATGCAATGTCAGTACAAGAATGGTCAAGCAATTCCAGATAAGGAGATT GCGCACATGATGATTGCCCTTCTCATGGCTGGTCAACACTCGTCCTCGTCCACCTCATGC TGGATCCTTCTCCGACTGGCTTCGCGACCAGATATCCAGGATGAGCTCCTTCAAGAACAG AAGGATGTGCTTGGCGTCAACGCAGATGGATCAATCAAGGAGTTAACGTACGCCGACATC TCGCGCCTTCCACTCCTCAATCAAGTTGTCAAGGAGACACTCCGCCTTCATGGTCCCATC CATTCTATTCTGCGACAAGTCAAGTCTCCGATGCCACTCGAAGGTACACCATACGTTGTC CCGACCACACACTCCCTCCTTGCTGCACCCGGTGCTACCTCACGAATGGACGAGCACTTC CCCGAAGCTATGCTGTGGGAACCCCACCGATGGGACGAGAACCCAAGTGAGAAGTACGCA CATCTCGCACCAAAGCATGTCAAGGAGGGCGTCGCCGAAGAGACTGAAGATTACGCCTAT GGTCTCGTCAGCAAAGGCGCCGCATCACCATATCTGCCATTCGGTGCTGGCCGACATAGA TGCATCGGCGAGCAATTCGCCTATGTCCAGCTCCAGACCATCACCTCGGAAGTGATTCGC GATTTCAAGCTCTACAATGTCGACGGCAGCGACAAAGTTGTCGGCACAGATTACAGTTCG TTGTTCAGCAGACCTCTCTCGCCAGCCGTCGTGCGATGGGAGAGGAGAGAAAAGAATA GATTTCACAGTA

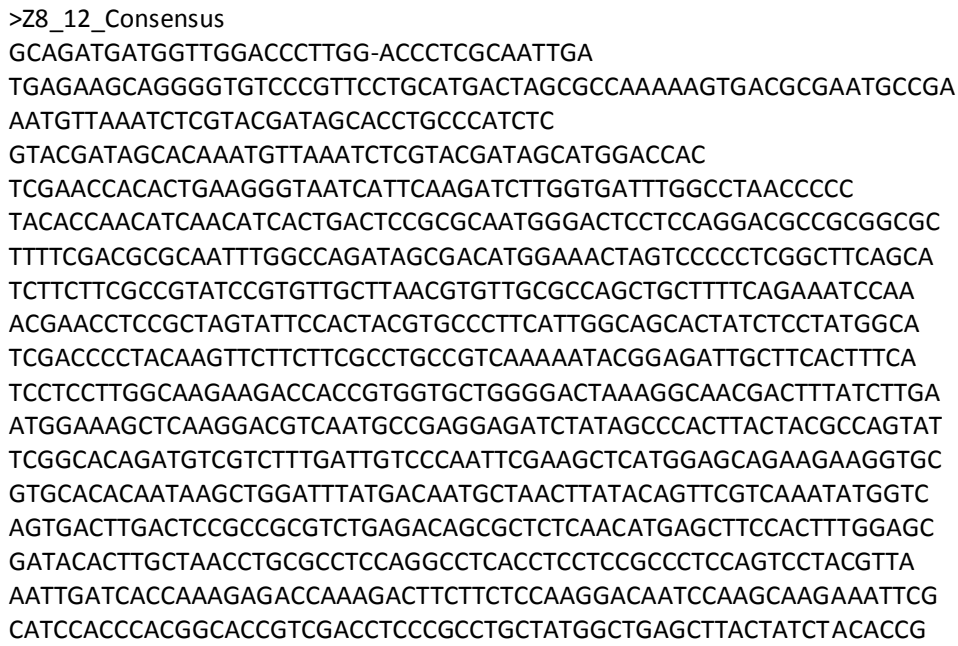
TCGGCACAGATGTCGTCTTTGATTGTCCCAATTCGAAGCTCATGGAGCAGAAGAAGGTGC GTGCACACAATAAGCTGGATTTATGACAATGCTAACTTATACAGTTCGTCAAATATGGTC AGTGACTTGACTCCGCCGCGTCTGAGACAGCGCTCTCAACATGAGCTTCCACTTTGGAGC GATACACTTGCTAACCTGCGCCTCCAGGCCTCACCTCCTCCGCCCTCCAGTCCTACGTTA AATTGATCACCAAAGAGACCAAAGACTTCTTCTCCAAGGACAATCCAAGCAAGAAATTCG CATCCACCCACGGCACCGTCGACCTCCCGCCTGCTATGGCTGAGCTTACTATCTACACCG 
CCAGCCGTTCGCTCCAGGGCAAAGAAGTCCGCGAAAAATTCGACTCCTCCTTTGCCGACC TCTACCACGATCTCGACATGGGCTTCACTCCCATCAACTTCATGCTTCCATGGGCTCCAC TGCCACAGAATAGAGCACGCGATCGCGCACAGAAGAAGATGGCGGAAGTCTACACAGCG ATCATCAAAGAGCGACGCGAAAAGGGCGAGCCTACTTCGGGAGAGAAAGAGCAGGACATG ATTTGGAATCTGATGCAATGTCAGTACAAGAATGGTCAAGCAATTCCAGATAAGGAGATT GCGCACATGATGATTGCCCTTCTCATGGCTGGTCAACACTCGTCCTCGTCCACCTCATGC TGGATCCTTCTCCGACTGGCTTCGCGACCAGATATCCAGGATGAGCTCCTTCAAGAACAG AAGGATGTGCTTGGCGTCAACGCAGATGGATCAATCAAGGAGTTAACGTACGCCGACATC TCGCGCCTTCCACTCCTCAATCAAGTTGTCAAGGAGACACTCCGCCTTCATGCTCCCATC CATTCTATTCTGCGACAAGTCAAGTCTCCGATGCCACTCGAAGGTACACCATACGTTGTC CCGACCACACACTCCCTCCTTGCTGCACCCGGTGCTACCTCACGAATGGACGAGCACTTC CCCGAAGCTATGCTGTGGGAACCCCACCGATGGGACGAGAACCCAAGTGAGAAGTACGCA CATCTCGCACCAAAGCATGTCAAGGAGGGCGTCGCCGAAGAGACTGAAGATTACGGCGAT GGTCTCGTCAGCAAAGGCGCCGCATCACCATATCTGCCATTCGGTGCTGGCCGACATAGA TGCATCGGCGAGCAATTCGCCTATGTCCAGCTCCAGACCATCACCTCGGAAGTGATTCGC GATTTCAAGCTCTACAATGTCGACGGCAGCGACAAAGTTGTCGGCACAGATTACAGTTCG TTGTTCAGCAGACCTCTCTCGCCAGCCGTCGTGCGATGGGAGAGGAGAGAAAAGAAATA GATTTCACAGTAAGA

\section{>Z8_17_Consensus} ATGATGGTTGGACCCTTGGACCCTCGCAATTGA

TGAGAAGCAGGGGTGTCCCGTTCCTGCATGACTAGCGCCAAAAAGTGACGCGAATGCCGA AATGTTAAATCTCGTACGATAGCACCTGCCCATGGACCAC

TCGAACCACACTGAAGGGTAATCATTCAAGATCTTGGTGATTTGGCCTAACCCCC TACACCAACATCAACATCACTGACTCCGCGCAATGGGACTCCTCCAGGACGCCGCGGCGC TTTTCGACGCGCAATTTGGCCAGATAGCGACATGGAAACTAGTCCCCCTCGGCTTCAGCA TCTTCTTCGCCGTATCCGTGTTGCTTAACGTGTTGCGCCAGCTGCTTTTCAGAAATCCAA ACGAACCTCCGCTAGTATTCCACTACGTGCCCTTCATTGGCAGCACTATCTCCTATGGCA TCGACCCCTACAAGTTCTTCTTCGCCTGCCGTCAAAAATACGGAGATTGCTTCACTTTCA TCCTCCTTGGCAAGAAGACCACCGTGGTGCTGGGGACTAAAGGCAACGACTTTATCTTGA ATGGAAAGCTCAAGGACGTCAATGCCGAGGAGATCTATAGCCCACTTACTACGCCAGTAT TCGGCACAGATGTCGTCTACGATTGTCCCAATTCGAAGCTCATGGAGCAGAAGAAGGTGC GTGCACACAATAAGCTGGATTTATGACAATGCTAACTTATACAGTTCGTCAAATATGGTC AGTGACTTGACTCCGCCGCGTCTGAGACAGCGCTCTCAACATGAGCTTCCACTTTGGAGC GATACACTTGCTAACCTGCGCCTCCAGGCCTCACCTCCTCCGCCCTCCAGTCCTACGTTA AATTGATCACCAAAGAGACCAAAGACTTCTTCTCCAAGGACAATCCAAGCAAGAAATTCG CATCCACCCACGGCACCGTCGACCTCCCGCCTGCTATGGCTGAGCTTACTATCTACACCG CCAGCCGTTCGCTCCAGGGCAAAGAAGTCCGCGAAAAATTCGACTCCTCCTTTGCCGACC TCTACCACGATCTCGACATGGGCTTCACTCCCATCAACTTCATGCTTCCATGGGCTCCAC TGCCACAGAATAGAGCACGCGATCGCGCACAGAAGAAGATGGCGGAAGTCTACACAGCG ATCATCAAAGAGCGACGCGAAAAGGGCGAGCCTACTTCGGGAGAGAAAGAGCAGGACATG ATTTGGAATCTGATGCAATGTCAGTACAAGAATGGTCAAGCAATTCCAGATAAGGAGATT GCGCACATGATGATTGCCCTTCTCATGGGTGGTCAACACTCGTCCTCGTCCACCTCATGC TGGATCCTTCTCCGACTGGCTTCGCGACCAGATATCCAGGATGAGCTCCTTCAAGAACAG AAGGATGTGCTTGGCGTCAACGCAGATGGATCAATCAAGGAGTTAACGTACGCCGACATC TCGCGCCTTCCACTCCTCAATCAAGTTGTCAAGGAGACACTCCGCCTTCATGCTCCCATC CATTCTATTCTGCGACAAGTCAAGTCTCCGATGCCACTCGAAGGTACACCATACGTTGTC CCGACCACACACTCCCTCCTTGCTGCACCCGGTGCTACCTCACGAATGGACGAGCACTTC CCCGAAGCTATGCTGTGGGAACCCCACCGATGGGACGAGAACCCAAGTGAGAAGTACGCA CATCTCGCACCAAAGCATGTCAAGGAGGGCGTCGCCGAAGAGACTGAAGATTACGGCTCT GGTCTCGTCAGCAAAGGCGCCGCATCACCATATCTGCCATTCGGTGCTGGCCGACATAGA TGCATCGGCGAGCAATTCGCCTATGTCCAGCTCCAGACCATCACCTCGGAAGTGATTCGC GATTTCAAGCTCTACAATGTCGACGGCAGCGACAAAGTTGTCGGCACAGATTACAGTTCG TTGTTCAGCAGACCTCTCTCGCCAGCCGTCGTGCGATGGGAGAGGAGAGAAAAGAAATA GATTTCACAGTAAGAG

$>$ Z8_18_Consensus

TTGG-ACCCTCGCAATTGA

TGAGAAGCAGGGGTGTCCCGTTCCTGCATGACTAGCGCCAAAAAGTGACGCGAATGCCGA AATGTTAAATCTCGTACGATAGCACCTGCCCATCTC GTACGATAGCACAAATGTTAAATCTCGTACGATAGCATGGACCAC TCGAACCACACTGAAGGGTAATCATTCAAGATCTTGGTGATTTGGCCTAACCCCC TACACCAACATCAACATCACTGACTCCGCGCAATGGGACTCCTCCAGGACGCCGCGGCGC TTTTCGACGCGCAATTTGGCCAGATAGCGACATGGAAACTAGTCCCCCTCGGCTTCAGCA TCTTCTTCGCCGTATCCGTGTTGCTTAACGTGTTGCGCCAGCTGCTTTTCAGAAATCCAA ACGAACCTCCGCTAGTATTCCACTACGTGCCCTTCATTGGCAGCACTATCTCCTATGGCA TCGACCCCTACAAGTTCTTCTTCGCCTGCCGTCAAAAATACGGAGATTGCTTCACTTTCA TCCTCCTTGGCAAGAAGACCACCGTGGTGCTGGGGACTAAAGGCAACGACTTTATCTTGA ATGGAAAGCTCAAGGACGTCAATGCCGAGGAGATCTATAGCCCACTTACTACGCCAGTAT 
TCGGCACAGATGTCGTCTTTGATTGTCCCAATTCGAAGCTCATGGAGCAGAAGAAGGTGC GTGCACACAATAAGCTGGATTTATGACAATGCTAACTTATACAGTTCGTCAAATATGGTC AGTGACTTGACTCCGCCGCGTCTGAGACAGCGCTCTCAACATGAGCTTCCACTTTGGAGC GATACACTTGCTAACCTGCGCCTCCAGGCCTCACCTCCTCCGCCCTCCAGTCCTACGTTA AATTGATCACCAAAGAGACCAAAGACTTCTTCTCCAAGGACAATCCAAGCAAGAAATTCG CATCCACCCACGGCACCGTCGACCTCCCGCCTGCTATGGCTGAGCTTACTATCTACACCG CCAGCCGTTCGCTCCAGGGCAAAGAAGTCCGCGAAAAATTCGACTCCTCCTTTGCCGACC TCTACCACGATCTCGACATGGGCTTCACTCCCATCAACTTCATGCTTCCATGGGCTCCAC TGCCACAGAATAGAGCACGCGATCGCGCACAGAAGAAGATGGCGGAAGTCTACACAGCG ATCATCAAAGAGCGACGCGAAAAGGGCGAGCCTACTTCGGGAGAGAAAGAGCAGGACATG ATTTGGAATCTGATGCAATGTCAGTACAAGAATGGTCAAGCAATTCCAGATAAGGAGATT GCGCACATGATGATTGCCCTTCTCATGGCTGGTCAACACTCGTCCTCGTCCACCTCATGC TGGATCCTTCTCCGACTGGCTTCGCGACCAGATATCCAGGATGAGCTCCTTCAAGAACAG AAGGATGTGCTTGGCGTCAACGCAGATGGATCAATCAAGGAGTTAACGTACGCCGACATC TCGCGCCTTCCACTCCTCAATCAAGTTGTCAAGGAGACACTCCGCCTTCATGCTCCCATC CATTCTATTCTGCGACAAGTCAAGTCTCCGATGCCACTCGAAGGTACACCATACGTTGTC CCGACCACACACTCCCTCCTTGCTGCACCCGGTGCTACCTCACGAATGGACGAGCACTTC CCCGAAGCTATGCTGTGGGAACCCCACCGATGGGACGAGAACCCAAGTGAGAAGTACGCA CATCTCGCACCAAAGCATGTCAAGGAGGGCGTCGCCGAAGAGACTGAAGATTACGGCGAT GGTCTCGTCAGCAAAGGCGCCGCATCACCATATCTGCCATTCGGTGCTGGCCGACATAGA TGCATCGGCGAGCAATTCGCCTATGTCCAGCTCCAGACCATCACCTCGGAAGTGATTCGC GATTTCAAGCTCTACAATGTCGACGGCAGCGACAAAGTTGTCGGCACAGATTACAGTTCG TTGTTCAGCAGACCTCTCTCGCCAGCCGTCGTGCGATGGGAGAGGAGAGAAAGAAATA GATTTCACAGTAAGAG

$>$ Ca1_5_Cyp51

CCAAAAAAGTGACGCGAATGCCGAAATGTTAAAATCTCGTACGATAGCACCTCCCCATCTCGTACGATAG CACAAATGTTAAATCTCGTACGATAGCATGGACCACTCGAACCCACACTGAAGGGTAATCATTCAAGATC TTGGTGATTTGGCCTAACCCCCTACACCAACATCAACATCACTGACTCCGCGCAATGGGACTCCTCCAGG ACGCCGCGGCGCTTTTCGACGCGCAATTTGGCCAGATAGCGACATGGAAACTAGTCCCCCTCGGCTTCAG CATCTTCTTCGCCGTATCCGTGTTGCTTAACGTGTTGCGCCAGCTGCTTTTCAGAAATCCAAACGAACCT CCGCTAGTATTCCACTACGTGCCCTTCATTGGCAGCACTATCTCCTATGGCATCGACCCCTACAAGTTCT TCTTCGCCTGCCGTCAAAAATACGGAGATTGCTTCACTTTCATCCTCCTTGGCAAGAAGACCACCGTGGT GCTGGGGACTAAAGGCAACGACTTTATCTTGAATGGAAAGCTCAAGGACGTCAATGCCGAGGAGATCTAT AGCCCACTTACTACGCCAGTATTCGGCACAGATGTCGTCTTTGATTGTCCCAATTCGAAGCTCATGGAGC AGAAGAAGGTGCGTGCACACAATAAGCTGGATTTATGACAATGCTAACTTATACAGTTCGTCAAATATGG TCAGTGACTTGACTCCGCCGCGTCTGAGACAGCGCTCTCAACATGAGCTTCCACTTTGGAGCGATACACT TGCTAACCTGCGCCTCCAGGCCTCACCTCCTCCGCCCTCCAGTCCTACGTTAAATTGATCACCAAAGAGA CCAAAGACTTCTTCTCCAAGGACAATCCAAGCAAGAAATTCGCATCCACCCACGGCACCGTCGACCTCCC GCCTGCTATGGCTGAGCTTACTATCTACACCGCCAGCCGTTCGCTCCAGGGCAAAGAAGTCCGCGAAAAA TTCGACTCCTCCTTTGCCGACCTCTACCACGATCTCGACATGGGCTTCACTCCCATCAACTTCATGCTTC CATGGGCTCCACTGCCACAGAATAGAGCACGCGATCGCGCACAGAAGAAGATGGCGGAAGTCTACACAGC GATCATCAAAGAGCGACGCGAAAAGGGCGAGCCTACTTCGGGAGAGAAAGAGCAGGACATGATTTGGAAT CTGATGCAATGTCAGTACAAGAATGGTCAAGCAATTCCAGATAAGGAGATTGCGCACATGATGATTGCCC TTCTCATGGCTGGTCAACACTCGTCCTCGTCCACCTCATGCTGGATCCTTCTCCGACTGGCTTCGCGACC AGATATCCAGGATGAGCTCCTTCAAGAACAGAAGGATGTGCTTGGCGTCAACGCAGATGGATCAATCAAG GAGTTAACGTACGCCGACATCTCGCGCCTTCCACTCCTCAATCAAGTTGTCAAGGAGACACTCCGCCTTC ATGGTCCCATCCATTCTATTCTGCGACAAGTCAAGTCTCCGATGCCACTCGAAGGTACACCATACGTTGT CCCGACCACACACTCCCTCCTTGCTGCACCCGGTGCTACCTCACGAATGGACGAGCACTTCCCCGAAGCT ATGCTGTGGGAACCCCACCGATGGGACGAGAACCCAAGTGAGAAGTACGCACATCTCGCACCAAAGCATG TCAAGGAGGGCGTCGCCGAAGAGACTGAAGATTACGGCGATGGTCTCGTCAGCAAAGGCGCCGCATCACC ATATCTGCCATTCGGTGCTGGCCGACATAGATGCATCGGCGAGCAATTCGCCTATGTCCAGCTCCAGACC ATCACCTCGGAAGTGATTCGCGATTTCAAGCTCTACAATGTCGACGGCAGCGACAAAGTTGTCGGCACAG ATTACAGTTCGTTGTTCAGCAGACCTCTCTCGCCAGCCGTCGTGCGATGGGAGAGGAGAGAAAAGAAATA GATTTCACAGTAAGAGTATGTTAATGCTAATCAGACAACTATATCCATATCTGCAGCTTCCCTCCATCTG TCACACGATAACATTCTATAAGAAGAACCAACCTGCAGCGCACTACCAACG

$>$ Ca5_16_Consensus

GAGATGGTGCAGATGATGGTTGGACCCTTGG-ACCCTCGCAATTGA

TGAGAAGCAGGGGTGTCCCGTTCCTGCATGACTAGCGCCAAAAAGTGACGCGAATGCCGA AATGTTAAATCTCGTACGATAGCACCTGCCCATAAATCTCGTACGATAGCATAAAATCTC GTACGATAGCATAAAATCTCGTACGATGTTAAATCTCGTACGATAGCATAAATCTCGTAC GATAGCACCTGCCCATTGAAGGGTAATCATTCAAGATCTTGGTGATTTGGCCTAACCCCC TACACCAACATCAACATCACTGACTCCGCGCAATGGGACTCCTCCAGGACGCCGCGGCGC TTTTCGACGCGCAATTTGGCCAGATAGCGACATGGAAACTAGTCCCCCTCGGCTTCAGCA TCTTCTTCGCCGTATCCGTGTTGCTTAACGTGTTGCGCCAGCTGCTTTTCAGAAATCCAA ACGAACCTCCGCTAGTATTCCACTACGTGCCCTTCATTGGCAGCACTATCTCCTATGGCA 
TCGACCCCTACAAGTTCTTCTTCGCCTGCCGTCAAAAATACGGAGATTGCTTCACTTTCA TCCTCCTTGGCAAGAAGACCACCGTGGTGCTGGGGACTAAAGGCAACGACTTTATCTTGA ATGGAAAGCTCAAGGACGTCAATGCCGAGGAGATCTATAGCCCACTTACTACGCCAGTAT TCGGCACAGATGTCGTCTTCGATTGTCCCAATTCGAAGCTCATGGAGCAGAAGAAGGTGC GTGCACACAATAAGCTGGATTTATGACAATGCTAACTTATACAGTTCGTCAAATATGGTC AGTGACTTGACTCCGCCGCGTCTGAGACAGCGCTCTCAACATGAGCTTCCACTTTGGAGC GATACACTTGCTAACCTGCGCCTCCAGGCCTCACCTCCTCCGCCCTCCAGTCCTACGTTA AATTGATCACCAAAGAGACCAAAGACTTCTTCTCCAAGGACAATCCAAGCAAGAAATTCG CATCCACCCACGGCACCGTCGACCTCCCGCCTGCTATGGCTGAGCTTACTATCTACACCG CCAGCCGTTCGCTCCAGGGCAAAGAAGTCCGCGAAAAATTCGACTCCTCCTTTGCCGACC TCTACCACGATCTCGACATGGGCTTCACTCCCATCAACTTCATGCTTCCATGGGCTCCAC TGCCACAGAA-TAGAGCACGCGATCGCGCACAGAAGAAGATGGCGGAAGTCTACACAGCG ATCATCAAAGAGCGACGCGAAAAGGGCGAGCCTACTTCGGGAGAGAAAGAGCAGGACATG ATTTGGAATCTGATGCAATGTCAGTACAAGAATGGTCAAGCAATTCCAGATAAGGAGATT GCGCACATGATGATTGCCCTTCTCATGGCTGGTCAACACTCGTCCTCGTCCACCTCATGC TGGATCCTTCTCCGACTGGCTTCGCGACCAGATATCCAGGATGAGCTCCTTCAAGAACAG AAGGATGTGCTTGGCGTCAACGCAGATGGATCAATCAAGGAGTTAACGTACGCCGACATC TCGCGCCTTCCACTCCTCAATCAAGTTGTCAAGGAGACACTCCGCCTTCATGCTCCCATC CATTCTATTCTGCGACAAGTCAAGTCTCCGATGCCACTCGAAGGTACACCATACGTTGTC CCGACCACACACTCCCTCCTTGCTGCACCCGGTGCTACCTCACGAATGGACGAGCACTTC CCCGAAGCTATGCTGTGGGAACCCCACCGATGGGACGAGAACCCAAGTGAGAAGTACGCA CATCTCGCACCAAAGCATGTCAAGGAGGGCGTCGCCGAAGAGACTGAAGATTACGGCGAT GGTCTCGTCAGCAAAGGCGCCGCATCACCATATCTGCCATTCGGTGCTGGCCGACATAGA TGCATCGGCGAGCAATTCGCCTATGTCCAGCTCCAGACCATCACCTCGGAAGTGATTCGC GATTTCAAGCTCTACAATGTCGACGGCAGCGACAAAGTTGTCGGCACAGATTACAGTTCG TTGTTCAGCAGACCTCTCTCGCCAGCCGTCGTGCGAT-GGGAGAGGAGAGAAAGAAA-TA GATTTCACAGTAAGAGTAT

$>$ Ca6_11-Consensus GCAGATGATGGTTGGACCCTTGG-ACCCTCGCAATTGA

TGAGAAGCAGGGGTGTCCCGTTCCTGCATGACTAGCGCCAAAAAGTGACGCGAATGCCGA AATGTTAAATCTCGTACGATAGCACCTGCCCATAAATCTCGTACGATAGCATAAAATCTC GTACGATAGCATAAAATCTCGTACGATGTTAAATCTCGTACGATAGCATAAATCTCGTAC GATAGCACCTGCCCATTGAAGGGTAATCATTCAAGATCTTGGTGATTTGGCCTAACCCCC TACACCAACATCAACATCACTGACTCCGCGCAATGGGACTCCTCCAGGACGCCGCGGCGC TTTTCGACGCGCAATTTGGCCAGATAGCGACATGGAAACTAGTCCCCCTCGGCTTCAGCA TCTTCTTCGCCGTATCCGTGTTGCTTAACGTGTTGCGCCAGCTGCTTTTCAGAAATCCAA ACGAACCTCCGCTAGTATTCCACTACGTGCCCTTCATTGGCAGCACTATCTCCTATGGCA TCGACCCCTACAAGTTCTTCTTCGCCTGCCGTCAAAAATACGGAGATTGCTTCACTTTCA TCCTCCTTGGCAAGAAGACCACCGTGGTGCTGGGGACTAAAGGCAACGACTTTATCTTGA ATGGAAAGCTCAAGGACGTCAATGCCGAGGAGATCTATAGCCCACTTACTACGCCAGTAT TCGGCACAGATGTCGTCTTCGATTGTCCCAATTCGAAGCTCATGGAGCAGAAGAAGGTGC GTGCACACAATAAGCTGGATTTATGACAATGCTAACTTATACAGTTCGTCAAATATGGTC AGTGACTTGACTCCGCCGCGTCTGAGACAGCGCTCTCAACATGAGCTTCCACTTTGGAGC GATACACTTGCTAACCTGCGCCTCCAGGCCTCACCTCCTCCGCCCTCCAGTCCTACGTTA AATTGATCACCAAAGAGACCAAAGACTTCTTCTCCAAGGACAATCCAAGCAAGAAATTCG CATCCACCCACGGCACCGTCGACCTCCCGCCTGCTATGGCTGAGCTTACTATCTACACCG CCAGCCGTTCGCTCCAGGGCAAAGAAGTCCGCGAAAAATTCGACTCCTCCTTTGCCGACC TCTACCACGATCTCGACATGGGCTTCACTCCCATCAACTTCATGCTTCCATGGGCTCCAC TGCCACAGAA-TAGAGCACGCGATCGCGCACAGAAGAAGATGGCGGAAGTCTACACAGCG ATCATCAAAGAGCGACGCGAAAAGGGCGAGCCTACTTCGGGAGAGAAAGAGCAGGACATG ATTTGGAATCTGATGCAATGTCAGTACAAGAATGGTCAAGCAATTCCAGATAAGGAGATT GCGCACATGATGATTGCCCTTCTCATGGCTGGTCAACACTCGTCCTCGTCCACCTCATGC TGGATCCTTCTCCGACTGGCTTCGCGACCAGATATCCAGGATGAGCTCCTTCAAGAACAG AAGGATGTGCTTGGCGTCAACGCAGATGGATCAATCAAGGAGTTAACGTACGCCGACATC TCGCGCCTTCCACTCCTCAATCAAGTTGTCAAGGAGACACTCCGCCTTCATGCTCCCATC CATTCTATTCTGCGACAAGTCAAGTCTCCGATGCCACTCGAAGGTACACCATACGTTGTC CCGACCACACACTCCCTCCTTGCTGCACCCGGTGCTACCTCACGAATGGACGAGCACTTC CCCGAAGCTATGCTGTGGGAACCCCACCGATGGGACGAGAACCCAAGTGAGAAGTACGCA CATCTCGCACCAAAGCATGTCAAGGAGGGCGTCGCCGAAGAGACTGAAGATTACGGCGAT GGTCTCGTCAGCAAAGGCGCCGCATCACCATATCTGCCATTCGGTGCTGGCCGACATAGA TGCATCGGCGAGCAATTCGCCTATGTCCAGCTCCAGACCATCACCTCGGAAGTGATTCGC GATTTCAAGCTCTACAATGTCGACGGCAGCGACAAAGTTGTCGGCACAGATTACAGTTCG TTGTTCAGCAGACCTCTCTCGCCAGCCGTCGTGCGAT-GGGAGAGGAGAGAAAAGAAATA $\mathrm{GA}$

$>$ Ca10_13

GATGGTGCAGATGATGGTTGGACCCTTGGACCCTCGCAATTGATGAGAAGCAGGGGTGTCCCGTTCCTGCATGACTAGC 
GCCAAAAAGTGACGCGAATGCCGAAATGTTAAATCTCGTACGATAGCACCTGCCCATAAATCTCGTACGATAGCATAAA ATCTCGTACGATAGCATAAAATCTCGTACGATGTTAAATCTCGTACGATAGCATAAATCTCGTACGATAGCACCTGCCC ATTGAAGGGTAATCATTCAAGATCTTGGTGATTTGGCCTAACCCCCTACACCAACATCAACATCACTGACTCCGCGCAA TGGGACTCCTCCAGGACGCCGCGGCGCTTTTCGACGCGCAATTTGGCCAGATAGCGACATGGAAACTAGTCCCCCTCGG CTTCAGCATCTTCTTCGCCGTATCCGTGTTGCTTAACGTGTTGCGCCAGCTGCTTTTCAGAAATCCAAACGAACCTCCG CTAGTATTCCACTACGTGCCCTTCATTGGCAGCACTATCTCCTATGGCATCGACCCCTACAAGTTCTTCTTCGCCTGCC GTCAAAAATACGGAGATTGCTTCACTTTCATCCTCCTTGGCAAGAAGACCACCGTGGTGCTGGGGACTAAAGGCAACGA CTTTATCTTGAATGGAAAGCTCAAGGACGTCAATGCCGAGGAGATCTATAGCCSACTTACTACGCCAGTATTCGGCACA GATGTCRTCTTCGATTGTCCCAATTCGAAGCTCATGGAGCGGAGGAAGGTGCGTGCACACAATAAGCTGGATTTATGAC AATGCTAACTTATACAGTTCGTCAAATATGGTCAGTGACTTGACTCCGCCGCGTCTGAGACAGCGCTCTCAACATGAGC TTCCACTTTGGAGCGATACACTTGCTAACCTGCGCCTCCAGGCCTCACCTCCTCCGCCCTCCAGTCCTACGTTAAATTG ATCACCAAAGAGACCAAAGACTTCTTCTCCAAGGACAATCCAAGCAAGAAATTCGCATCCACCCACGGCACCGTCGACC TCCCGCCTGCTATGGCTGAGCTTACTATCTACACCGCCAGCCGTTCGCTCCAGGGCAAAGAAGTCCGCGAAAAATTCGA CTCCTCCTTTGCCGACCTCTACCACGATCTCGACATGGGCTTCACTCCCATCAACTTCATGCTTCCATGGGCTCCACTG CCACAGAATAGAGCACGCGATCGCGCACAGAAGAAGATGGCGGAAGTCTACACAGCGATCATCAAAGAGCGACGCGAAA AGGGCGAGCCTACTTCGGGAGAGAAAGAGCAGGACATGATTTGGAATCTGATGCAaTGTCAGTACAAGAATGGTCAAGC AATTCCAGATAAGGAGATTGCGCACATGATGATTGCCCTTCTCATGGCTGGTCAACACTCGTCCTCGTCCACCTCATGC TGGATCCTTCTCCGACTGGCTTCGCGACCAGATATCCAGGATGAGCTCCTTCAAGAACAGAAGGATGTGCTTGGCGTCA ACGCAGATGGATCAATCAAGGAGTTAACGTACGCCGACATCTCGCGCCTTCCACTCCTCAATCAAGTTGTCAAGGAGAC ACTCCGCCTTCATGCTCCCATCCATTCTATTCTGCGACAAGTCAAGTCTCCGATGCCACTCGAAGGTACACCATACGTT GTCCCGACCACACACTCCCTCCTTGCTGCACCCGGTGCTACCTCACGAATGGACGAGCACTTCCCCGAAGCTATGCTGT GGGAACCCCACCGATGGGACGAGAACCCAAGTGAGAAGTACGCACATCTCGCACCAAAGCATGTCAAGGAGGGCGTCGC CGAAGAGACTGAAGATTACGGCGATGGTCTCGTCAGCAAAGGCGCCGCATCACCATATCTGCCATTCGGTGCTGGCCGA CATAGATGCATCGGCGAGCAATTCGCCTATGTCCAGCTCCAGACCATCACCTCGGAAGTGATTCGCGATTTCAAGCTCT ACAATGTCGACGGCAGCGACAAAGTTGTCGGCACAGATTACAGTTCGTTGTTCAGCAGACCTCTCTCGCCAGCCGTCGT GCGATGGGAGAGGAGAGAAAAGAAATAGATTCACAGTAAGGA

\begin{abstract}
$>$ E_22
AGATGGTGCAGATGATGGTTGGACCCTTGGACCCTCGCAATTGATGAGAAGCAGGGGTGTCCCGTTCCTGCATGACTAG CGCCAAAAAGTGACGCGAATGCCGAAATGTTAAATCTCGTACGATAGCACCTGCCCATGGACCACTCGAACCACACTGA AGGGTAATCATTCAAGATCTTGGTGATTTGGCCTAACCCCCTACACCAACATCAACATCACTGACTCCGCGCAATGGGA CTCCTCCAGGACGCCGCGGCGCTTTTCGACGCGCAATTTGGCCAGATAGCGACATGGAAACTAGTCCCCCTCGGCTTCA GCATCTTCTTCGCCGTATCCGTGTTGCTTAACGTGTTGCGCCAGCTGCTTTTCAGAAATCCAAACGAACCTCCGCTAGT ATTCCACTACGTGCCCTTCATTGGCAGCACTATCTCCTATGGCATCGACCCCTACAAGTTCTTCTTCGCCTGCCGTCAA AAATACGGAGATTGCTTCACTTTCATCCTCCTTGGCAAGAAGACCACCGTGGTGCTGGGGACTAAAGGCAACGACTTTA TCTTGAATGGAAAGCTCAAGGACGTCAATGCCGAGGAGATCTATAGCCCACTTACTACGCCAGTATTCGGCACAGATGT CGTCTACGATTGTCCCAATTCGAAGCTCATGGAGCAGAAGAAGGTGCGTGCACACAATAAGCTGGATTTATGACAATGC TAACTTATACAGTTCGTCAAATATGGTCAGTGACTTGACTCCGCCGCGTCTGAGACAGCGCTCTCAACATGAGCTTCCA CTTTGGAGCGATACACTTGCTAACCTGCGCCTCCAGGCCTCACCTCCTCCGCCCTCCAGTCCTACGTTAAATTGATCAC CAAAGAGACCAAAGACTTCTTCTCCAAGGACAATCCAAGCAAGAAATTCGCATCCACCCACGGCACCGTCGACCTCCCG CCTGCTATGGCTGAGCTTACTATCTACACCGCCAGCCGTTCGCTCCAGGGCAAAGAAGTCCGCGAAAAATTCGACTCCT CCTTTGCCGACCTCTACCACGATCTCGACATGGGCTTCACTCCCATCAACTTCATGCTTCCATGGGCTCCACTGCCACA GAATAGAGCACGCGATCGCGCACAGAAGAAGATGGCGGAAGTCTACACAGCGATCATCAAAGAGCGACGCGAAAAGGGC GAGCCTACTTCGGGAGAGAAAGAGCAGGACATGATTTGGAATCTGATGCAATGTCAGTACAAGAATGGTCAAGCAATTC CAGATAAGGAGATTGCGCACATGATGATTGCCCTTCTCATGGCTGGTCAACACTCGTCCTCGTCCACCTCATGCTGGAT CCTTCTCCGACTGGCTTCGCGACCAGATATCCAGGATGAGCTCCTTCAAGAACAGAAGGATGTGCTTGGCGTCAACGCA GATGGATCAATCAAGGAGTTAACGTACGCCGACATCTCGCGCCTTCCACTCCTCAATCAAGTTGTCAAGGAGACACTCC GCCTTCATGCTCCCATCCATTCTATTCTGCGACAAGTCAAGTCTCCGATGCCACTCGAAGGTACACCATACGTTGTCCC GACCACACACTCCCTCCTTGCTGCACCCGGTGCTACCTCACGAATGGACGAGCACTTCCCCGAAGCTATGCTGTGGGAA CCCCACCGATGGGACGAGAACCCAAGTGAGAAGTACGCACATCTCGCACCAAAGCATGTCAAGGAGGGCGTCGCCGAAG AGACTGAAGATTACGGCTATGGTCTCGTCAGCAAAGGCGCCGCATCACCATATCTGCCATTCGGTGCTGGCCGACATAG ATGCATCGGCGAGCAATTCGCCTATGTCCAGCTCCAGACCATCACCTCGGAAGTGATTCGCGATTTCAAGCTCTACAAT GTCGACGGCAGCGACAAAGTTGTCGGCACAGATTACAGTTCGTTGTTCAGCAGACCTCTCTCGCCAGCCGTCGTGCGAT GGGAGAGGAGAGAAAAGAAATAGATCACAGTAAA
\end{abstract}

$>$ GS_10-Consensus

CGAGATGGTGCAGATGATGGTTGGACCCTTGGACCCTCGCAATTGA

TGAGAAGCAGGGGTGTCCCGTTCCTGCATGACTAGCGCCAAAAAGTGACGCGAATGCCGA

AATGTTAAATCTCGTACGATAGCACCTGCCCATGGACCAC

TCGAACCACACTGAAGGGTAATCATTCAAGATCTTGGTGATTTGGCCTAACCCCC

TACACCAACATCAACATCACTGACTCCGCGCAATGGGACTCCTCCAGGACGCCGCGGCGC

TTTTCGACGCGCAATTTGGCCAGATAGCGACATGGAAACTAGTCCCCCTCGGCTTCAGCA

TCTTCTTCGCCGTATCCGTGTTGCTTAACGTGTTGCGCCAGCTGCTTTTTCAGAAATCCAA 
ACGAACCTCCGCTAGTATTCCACTACGTGCCCTTCATTGGCAGCACTATCTCCTATGGCA TCGACCCCTACAAGTTCTTCTTCGCCTGCCGTCAAAAATACGGAGATTGCTTCACTTTCA TCCTCCTTGGCAAGAAGACCACCGTGGTGCTGGGGACTAAAGGCAACGACTTTATCTTGA ATGGAAAGCTCAAGGACGTCAATGCCGAGGAGATCTATAGCCCACTTACTACGCCAGTAT TCGGCACAGATGTCGTCTACGATTGTCCCAATTCGAAGCTCATGGAGCAGAAGAAGGTGC GTGCACACAATAAGCTGGATTTATGACAATGCTAACTTATACAGTTCGTCAAATATGGTC AGTGACTTGACTCCGCCGCGTCTGAGACAGCGCTCTCAACATGAGCTTCCACTTTGGAGC GATACACTTGCTAACCTGCGCCTCCAGGCCTCACCTCCTCCGCCCTCCAGTCCTACGTTA AATTGATCACCAAAGAGACCAAAGACTTCTTCTCCAAGGACAATCCAAGCAAGAAATTCG CATCCACCCACGGCACCGTCGACCTCCCGCCTGCTATGGCTGAGCTTACTATCTACACCG CCAGCCGTTCGCTCCAGGGCAAAGAAGTCCGCGAAAAATTCGACTCCTCCTTTGCCGACC TCTACCACGATCTCGACATGGGCTTCACTCCCATCAACTTCATGCTTCCATGGGCTCCAC TGCCACAGAATAGAGCACGCGATCGCGCACAGAAGAAGATGGCGGAAGTCTACACAGCG ATCATCAAAGAGCGACGCGAAAAGGGCGAGCCTACTTCGGGAGAGAAAGAGCAGGACATG ATTTGGAATCTGATGCAATGTCAGTACAAGAATGGTCAAGCAATTCCAGATAAGGAGATT GCGCACATGATGATTGCCCTTCTCATGGGTGGTCAACACTCGTCCTCGTCCACCTCATGC TGGATCCTTCTCCGACTGGCTTCGCGACCAGATATCCAGGATGAGCTCCTTCAAGAACAG AAGGATGTGCTTGGCGTCAACGCAGATGGATCAATCAAGGAGTTAACGTACGCCGACATC TCGCGCCTTCCACTCCTCAATCAAGTTGTCAAGGAGACACTCCGCCTTCATGCTCCCATC CATTCTATTCTGCGACAAGTCAAGTCTCCGATGCCACTCGAAGGTACACCATACGTTGTC CCGACCACACACTCCCTCCTTGCTGCACCCGGTGCTACCTCACGAATGGACGAGCACTTC CCCGAAGCTATGCTGTGGGAACCCCACCGATGGGACGAGAACCCAAGTGAGAAGTACGCA CATCTCGCACCAAAGCATGTCAAGGAGGGCGTCGCCGAAGAGACTGAAGATTACGGCAAT GGTCTCGTCAGCAAAGGCGCCGCATCACCATATCTGCCATTCGGTGCTGGCCGACATAGA TGCATCGGCGAGCAATTCGCCTATGTCCAGCTCCAGACCATCACCTCGGAAGTGATTCGC GATTTCAAGCTCTACAATGTCGACGGCAGCGACAAAGTTGTCGGCACAGATTACAGTTCG TTGTTCAGCAGACCTCTCTCGCCAGCCGTCGTGCGATGGGAGAGGAGAGAAAGAAATA GATTTCACAGTA

>RN_3-Consensus

CGAGATGGTGCAGATGATGGTTGGACCCTTG-GACCCTCGCAATTGA TGAGAAGCAGGGGTGTCCCGTTCCTGCATGACTAGCGCCAAAAAGTGACGCGAATGCCGA AATGTTAAATCTCGTACGATAGCACCTGCCCATGGACCAC

TCGAACCACACTGAAGGGTAATCATTCAAGATCTTGGTGATTTGGCCTAACCCCC

TACACCAACATCAACATCACTGACTCCGCGCAATGGGACTCCTCCAGGACGCCGCGGCGC TTTTCGACGCGCAATTTGGCCAGATAGCGACATGGAAACTAGTCCCCCTCGGCTTCAGCA TCTTCTTCGCCGTATCCGTGTTGCTTAACGTGTTGCGCCAGCTGCTTTTCAGAAATCCAA ACGAACCTCCGCTAGTATTCCACTACGTGCCCTTCATTGGCAGCACTATCTCCTATGGCA TCGACCCCTACAAGTTCTTCTTCGCCTGCCGTCAAAAATACGGAGATTGCTTCACTTTCA TCCTCCTTGGCAAGAAGACCACCGTGGTGCTGGGGACTAAAGGCAACGACTTTATCTTGA ATGGAAAGCTCAAGGACGTCAATGCCGAGGAGATCTATAGCCCACTTACTACGCCAGTAT TCGGCACAGATGTCGTCTACGATTGTCCCAATTCGAAGCTCATGGAGCAGAAGAAGGTGC GTGCACACAATAAGCTGGATTTATGACAATGCTAACTTATACAGTTCGTCAAATATGGTC AGTGACTTGACTCCGCCGCGTCTGAGACAGCGCTCTCAACATGAGCTTCCACTTTGGAGC GATACACTTGCTAACCTGCGCCTCCAGGCCTCACCTCCTCCGCCCTCCAGTCCTACGTTA AATTGATCACCAAAGAGACCAAAGACTTCTTCTCCAAGGACAATCCAAGCAAGAAATTCG CATCCACCCACGGCACCGTCGACCTCCCGCCTGCTATGGCTGAGCTTACTATCTACACCG CCAGCCGTTCGCTCCAGGGCAAAGAAGTCCGCGAAAAATTCGACTCCTCCTTTGCCGACC TCTACCACGATCTCGACATGGGCTTCACTCCCATCAACTTCATGCTTCCATGGGCTCCAC TGCCACAGAA-TAGAGCACGCGATCGCGCACAGAAGAAGATGGCGGAAGTCTACACAGCG ATCATCAAAGAGCGACGCGAAAAGGGCGAGCCTACTTCGGGAGAGAAAGAGCAGGACATG ATTTGGAATCTGATGCAATGTCAGTACAAGAATGGTCAAGCAATTCCAGATAAGGAGATT GCGCACATGATGATTGCCCTTCTCATGGGTGGTCAACACTCGTCCTCGTCCACCTCATGC TGGATCCTTCTCCGACTGGCTTCGCGACCAGATATCCAGGATGAGCTCCTTCAAGAACAG AAGGATGTGCTTGGCGTCAACGCAGATGGATCAATCAAGGAGTTAACGTACGCCGACATC TCGCGCCTTCCACTCCTCAATCAAGTTGTCAAGGAGACACTCCGCCTTCATGCTCCCATC CATTCTATTCTGCGACAAGTCAAGTCTCCGATGCCACTCGAAGGTACACCATACGTTGTC CCGACCACACACTCCCTCCTTGCTGCACCCGGTGCTACCTCACGAATGGACGAGCACTTC CCCGAAGCTATGCTGTGGGAACCCCACCGATGGGACGAGAACCCAAGTGAGAAGTACGCA CATCTCGCACCAAAGCATGTCAAGGAGGGCGTCGCCGAAGAGACTGAAGATTACGGCCAT GGTCTCGTCAGCAAAGGCGCCGCATCACCATATCTGCCATTCGGTGCTGGCCGACATAGA TGCATCGGCGAGCAATTCGCCTATGTCCAGCTCCAGACCATCACCTCGGAAGTGATTCGC GATTTCAAGCTCTACAATGTCGACGGCAGCGACAAAGTTGTCGGCACAGATTACAGTTCG TTGTTCAGCAGACCTCTCTCGCCAGCCGTCGTGCGATGGGAGAGGAGAGAAAGAATA GATTTCACAGTAAGA 
CGAGATGGTGCAGATGATGGTTGGACCCTTGGACCCTCGCAATTGA

TGAGAAGCAGGGGTGTCCCGTTCCTGCATGACTAGCGCCAAAAAGTGACGCGAATGCCGA AATGTTAAATCTCGTACGATAGCACCTGCCCATGGACCAC

TCGAACCACACTGAAGGGTAATCATTCAAGATCTTGGTGATTTGGCCTAACCCCC

TACACCAACATCAACATCACTGACTCCGCGCAATGGGACTCCTCCAGGACGCCGCGGCGC TTTTCGACGCGCAATTTGGCCAGATAGCGACATGGAAACTAGTCCCCCTCGGCTTCAGCA TCTTCTTCGCCGTATCCGTGTTGCTTAACGTGTTGCGCCAGCTGCTTTTCAGAAATCCAA ACGAACCTCCGCTAGTATTCCACTACGTGCCCTTCATTGGCAGCACTATCTCCTATGGCA TCGACCCCTACAAGTTCTTCTTCGCCTGCCGTCAAAAATACGGAGATTGCTTCACTTTCA TCCTCCTTGGCAAGAAGACCACCGTGGTGCTGGGGACTAAAGGCAACGACTTTATCTTGA ATGGAAAGCTCAAGGACGTCAATGCCGAGGAGATCTATAGCCCACTTACTACGCCAGTAT TCGGCACAGATGTCGTCTACGATTGTCCCAATTCGAAGCTCATGGAGCAGAAGAAGGTGC GTGCACACAATAAGCTGGATTTATGACAATGCTAACTTATACAGTTCGTCAAATATGGTC AGTGACTTGACTCCGCCGCGTCTGAGACAGCGCTCTCAACATGAGCTTCCACTTTGGAGC GATACACTTGCTAACCTGCGCCTCCAGGCCTCACCTCCTCCGCCCTCCAGTCCTACGTTA AATTGATCACCAAAGAGACCAAAGACTTCTTCTCCAAGGACAATCCAAGCAAGAAATTCG CATCCACCCACGGCACCGTCGACCTCCCGCCTGCTATGGCTGAGCTTACTATCTACACCG CCAGCCGTTCGCTCCAGGGCAAAGAAGTCCGCGAAAAATTCGACTCCTCCTTTGCCGACC TCTACCACGATCTCGACATGGGCTTCACTCCCATCAACTTCATGCTTCCATGGGCTCCAC TGCCACAGAATAGAGCACGCGATCGCGCACAGAAGAAGATGGCGGAAGTCTACACAGCG ATCATCAAAGAGCGACGCGAAAAGGGCGAGCCTACTTCGGGAGAGAAAGAGCAGGACATG ATTTGGAATCTGATGCAATGTCAGTACAAGAATGGTCAAGCAATTCCAGATAAGGAGATT GCGCACATGATGATTGCCCTTCTCATGGGTGGTCAACACTCGTCCTCGTCCACCTCATGC TGGATCCTTCTCCGACTGGCTTCGCGACCAGATATCCAGGATGAGCTCCTTCAAGAACAG AAGGATGTGCTTGGCGTCAACGCAGATGGATCAATCAAGGAGTTAACGTACGCCGACATC TCGCGCCTTCCACTCCTCAATCAAGTTGTCAAGGAGACACTCCGCCTTCATGCTCCCATC CATTCTATTCTGCGACAAGTCAAGTCTCCGATGCCACTCGAAGGTACACCATACGTTGTC CCGACCACACACTCCCTCCTTGCTGCACCCGGTGCTACCTCACGAATGGACGAGCACTTC CCCGAAGCTATGCTGTGGGAACCCCACCGATGGGACGAGAACCCAAGTGAGAAGTACGCA CATCTCGCACCAAAGCATGTCAAGGAGGGCGTCGCCGAAGAGACTGAAGATTACGGCCAT GGTCTCGTCAGCAAAGGCGCCGCATCACCATATCTGCCATTCGGTGCTGGCCGACATAGA TGCATCGGCGAGCAATTCGCCTATGTCCAGCTCCAGACCATCACCTCGGAAGTGATTCGC GATTTCAAGCTCTACAATGTCGACGGCAGCGACAAAGTTGTCGGCACAGATTACAGTTCG TTGTTCAGCAGACCTCTCTCGCCAGCCGTCGTGCGATGGGAGAGGAGAGAAAGAATA GATTTCACAGTAAGAGTATGTTAATGCTAATCA

\section{$>$ RS_13-Consensus}

TCGCAATTGA

TGAGAAGCAGGGGTGTCCCGTTCCTGCATGACTAGCGCCAAAAAGTGACGCGAATGCCGA AATGTTAAATCTCGTACGATAGCACCTGCCCATGGACCAC

TCGAACCACACTGAAGGGTAATCATTCAAGATCTTGGTGATTTGGCCTAACCCCC

TACACCAACATCAACATCACTGACTCCGCGCAATGGGACTCCTCCAGGACGCCGCGGCGC TTTTCGACGCGCAATTTGGCCAGATAGCGACATGGAAACTAGTCCCCCTCGGCTTCAGCA TCTTCTTCGCCGTATCCGTGTTGCTTAACGTGTTGCGCCAGCTGCTTTTCAGAAATCCAA ACGAACCTCCGCTAGTATTCCACTACGTGCCCTTCATTGGCAGCACTATCTCCTATGGCA TCGACCCCTACAAGTTCTTCTTCGCCTGCCGTCAAAAATACGGAGATTGCTTCACTTTCA TCCTCCTTGGCAAGAAGACCACCGTGGTGCTGGGGACTAAAGGCAACGACTTTATCTTGA ATGGAAAGCTCAAGGACGTCAATGCCGAGGAGATCTATAGCCCACTTACTACGCCAGTAT TCGGCACAGATGTCGTCTACGATTGTCCCAATTCGAAGCTCATGGAGCAGAAGAAGGTGC GTGCACACAATAAGCTGGATTTATGACAATGCTAACTTATACAGTTCGTCAAATATGGTC AGTGACTTGACTCCGCCGCGTCTGAGACAGCGCTCTCAACATGAGCTTCCACTTTGGAGC GATACACTTGCTAACCTGCGCCTCCAGGCCTCACCTCCTCCGCCCTCCAGTCCTACGTTA AATTGATCACCAAAGAGACCAAAGACTTCTTCTCCAAGGACAATCCAAGCAAGAAATTCG CATCCACCCACGGCACCGTCGACCTCCCGCCTGCTATGGCTGAGCTTACTATCTACACCG CCAGCCGTTCGCTCCAGGGCAAAGAAGTCCGCGAAAAATTCGACTCCTCCTTTGCCGACC TCTACCACGATCTCGACATGGGCTTCACTCCCATCAACTTCATGCTTCCATGGGCTCCAC TGCCACAGAA-TAGAGCACGCGATCGCGCACAGAAGAAGATGGCGGAAGTCTACACAGCG ATCATCAAAGAGCGACGCGAAAAGGGCGAGCCTACTTCGGGAGAGAAAGAGCAGGACATG ATTTGGAATCTGATGCAATGTCAGTACAAGAATGGTCAAGCAATTCCAGATAAGGAGATT GCGCACATGATGATTGCCCTTCTCATGGGTGGTCAACACTCGTCCTCGTCCACCTCATGC TGGATCCTTCTCCGACTGGCTTCGCGACCAGATATCCAGGATGAGCTCCTTCAAGAACAG AAGGATGTGCTTGGCGTCAACGCAGATGGATCAATCAAGGAGTTAACGTACGCCGACATC TCGCGCCTTCCACTCCTCAATCAAGTTGTCAAGGAGACACTCCGCCTTCATGCTCCCATC CATTCTATTCTGCGACAAGTCAAGTCTCCGATGCCACTCGAAGGTACACCATACGTTGTC CCGACCACACACTCCCTCCTTGCTGCACCCGGTGCTACCTCACGAATGGACGAGCACTTC CCCGAAGCTATGCTGTGGGAACCCCACCGATGGGACGAGAACCCAAGTGAGAAGTACGCA CATCTCGCACCAAAGCATGTCAAGGAGGGCGTCGCCGAAGAGACTGAAGATTACGGCAAT GGTCTCGTCAGCAAAGGCGCCGCATCACCATATCTGCCATTCGGTGCTGGCCGACATAGA TGCATCGGCGAGCAATTCGCCTATGTCCAGCTCCAGACCATCACCTCGGAAGTGATTCGC 


\title{
CHAPTER 4
}

GATTTCAAGCTCTACAATGTCGACGGCAGCGACAAAGTTGTCGGCACAGATTACAGTTCG TTGTTCAGCAGACCTCTCTCGCCAGCCGTCGTGCGATGGGAGAGGAGAGAAAAGAAATA GATTTCACAGTAGAGTATG

\begin{abstract}
$>$ SaR 2-Consensus
GAGATGGTGCAGATGATGGTTGGACCCTTG-GACCCTCGCAATTGA

TGAGAAGCAGGGGTGTCCCGTTCCTGCATGACTAGCGCCAAAAAGTGACGCGAATGCCGA AATGTTAAATCTCGTACGATAGCACCTGCCCATGGACCAC

TCGAACCACACTGAAGGGTAATCATTCAAGATCTTGGTGATTTGGCCTAACCCCC

TACACCAACATCAACATCACTGACTCCGCGCAATGGGACTCCTCCAGGACGCCGCGGCGC TTTTCGACGCGCAATTTGGCCAGATAGCGACATGGAAACTAGTCCCCCTCGGCTTCAGCA TCTTCTTCGCCGTATCCGTGTTGCTTAACGTGTTGCGCCAGCTGCTTTTCAGAAATCCAA ACGAACCTCCGCTAGTATTCCACTACGTGCCCTTCATTGGCAGCACTATCTCCTATGGCA TCGACCCCTACAAGTTCTTCTTCGCCTGCCGTCAAAAATACGGAGATTGCTTCACTTTCA TCCTCCTTGGCAAGAAGACCACCGTGGTGCTGGGGACTAAAGGCAACGACTTTATCTTGA ATGGAAAGCTCAAGGACGTCAATGCCGAGGAGATCTATAGCCCACTTACTACGCCAGTAT TCGGCACAGATGTCGTCTACGATTGTCCCAATTCGAAGCTCATGGAGCAGAAGAAGGTGC GTGCACACAATAAGCTGGATTTATGACAATGCTAACTTATACAGTTCGTCAAATATGGTC AGTGACTTGACTCCGCCGCGTCTGAGACAGCGCTCTCAACATGAGCTTCCACTTTGGAGC GATACACTTGCTAACCTGCGCCTCCAGGCCTCACCTCCTCCGCCCTCCAGTCCTACGTTA AATTGATCACCAAAGAGACCAAAGACTTCTTCTCCAAGGACAATCCAAGCAAGAAATTCG CATCCACCCACGGCACCGTCGACCTCCCGCCTGCTATGGCTGAGCTTACTATCTACACCG CCAGCCGTTCGCTCCAGGGCAAAGAAGTCCGCGAAAAATTCGACTCCTCCTTTGCCGACC TCTACCACGATCTCGACATGGGCTTCACTCCCATCAACTTCATGCTTCCATGGGCTCCAC TGCCACAGAA-TAGAGCACGCGATCGCGCACAGAAGAAGATGGCGGAAGTCTACACAGCG ATCATCAAAGAGCGACGCGAAAAGGGCGAGCCTACTTCGGGAGAGAAAGAGCAGGACATG ATTTGGAATCTGATGCAATGTCAGTACAAGAATGGTCAAGCAATTCCAGATAAGGAGATT GCGCACATGATGATTGCCCTTCTCATGGGTGGTCAACACTCGTCCTCGTCCACCTCATGC TGGATCCTTCTCCGACTGGCTTCGCGACCAGATATCCAGGATGAGCTCCTTCAAGAACAG AAGGATGTGCTTGGCGTCAACGCAGATGGATCAATCAAGGAGTTAACGTACGCCGACATC TCGCGCCTTCCACTCCTCAATCAAGTTGTCAAGGAGACACTCCGCCTTCATGCTCCCATC CATTCTATTCTGCGACAAGTCAAGTCTCCGATGCCACTCGAAGGTACACCATACGTTGTC CCGACCACACACTCCCTCCTTGCTGCACCCGGTGCTACCTCACGAATGGACGAGCACTTC CCCGAAGCTATGCTGTGGGAACCCCACCGATGGGACGAGAACCCAAGTGAGAAGTACGCA CATCTCGCACCAAAGCATGTCAAGGAGGGCGTCGCCGAAGAGACTGAAGATGACGGCTAT GGTCTCGTCAGCAAAGGCGCCGCATCACCATATCTGCCATTCGGTGCTGGCCGACATAGA TGCATCGGCGAGCAATTCGCCTATGTCCAGCTCCAGACCATCACCTCGGAAGTGATTCGC GATTTCAAGCTCTACAATGTCGACGGCAGCGACAAAGTTGTCGGCACAGATTACAGTTCG TTGTTCAGCAGACCTCTCTCGCCAGCCGTCGTGCGATGGGAGAGGAGAGAAAGAAATA GATTTCACAGTAAGAG
\end{abstract}

$>$ SaR_5-Consensus

GGTGCAGATGATGGTTGGACCCTTG-GACCCTCGCAATTGA

TGAGAAGCAGGGGTGTCCCGTTCCTGCATGACTAGCGCCAAAAAGTGACGCGAATGCCGA AATGTTAAATCTCGTACGATAGCACCTGCCCATGGACCAC

TCGAACCACACTGAAGGGTAATCATTCAAGATCTTGGTGATTTGGCCTAACCCCC

TACACCAACATCAACATCACTGACTCCGCGCAATGGGACTCCTCCAGGACGCCGCGGCGC TTTTCGACGCGCAATTTGGCCAGATAGCGACATGGAAACTAGTCCCCCTCGGCTTCAGCA TCTTCTTCGCCGTATCCGTGTTGCTTAACGTGTTGCGCCAGCTGCTTTTCAGAAATCCAA ACGAACCTCCGCTAGTATTCCACTACGTGCCCTTCATTGGCAGCACTATCTCCTATGGCA TCGACCCCTACAAGTTCTTCTTCGCCTGCCGTCAAAAATACGGAGATTGCTTCACTTTCA TCCTCCTTGGCAAGAAGACCACCGTGGTGCTGGGGACTAAAGGCAACGACTTTATCTTGA ATGGAAAGCTCAAGGACGTCAATGCCGAGGAGATCTATAGCCCACTTACTACGCCAGTAT TCGGCACAGATGTCGTCTACGATTGTCCCAATTCGAAGCTCATGGAGCAGAAGAAGGTGC GTGCACACAATAAGCTGGATTTATGACAATGCTAACTTATACAGTTCGTCAAATATGGTC AGTGACTTGACTCCGCCGCGTCTGAGACAGCGCTCTCAACATGAGCTTCCACTTTGGAGC GATACACTTGCTAACCTGCGCCTCCAGGCCTCACCTCCTCCGCCCTCCAGTCCTACGTTA AATTGATCACCAAAGAGACCAAAGACTTCTTCTCCAAGGACAATCCAAGCAAGAAATTCG CATCCACCCACGGCACCGTCGACCTCCCGCCTGCTATGGCTGAGCTTACTATCTACACCG CCAGCCGTTCGCTCCAGGGCAAAGAAGTCCGCGAAAAATTCGACTCCTCCTTTGCCGACC TCTACCACGATCTCGACATGGGCTTCACTCCCATCAACTTCATGCTTCCATGGGCTCCAC TGCCACAGAA-TAGAGCACGCGATCGCGCACAGAAGAAGATGGCGGAAGTCTACACAGCG ATCATCAAAGAGCGACGCGAAAAGGGCGAGCCTACTTCGGGAGAGAAAGAGCAGGACATG ATTTGGAATCTGATGCAATGTCAGTACAAGAATGGTCAAGCAATTCCAGATAAGGAGATT GCGCACATGATGATTGCCCTTCTCATGGGTGGTCAACACTCGTCCTCGTCCACCTCATGC TGGATCCTTCTCCGACTGGCTTCGCGACCAGATATCCAGGATGAGCTCCTTCAAGAACAG AAGGATGTGCTTGGCGTCAACGCAGATGGATCAATCAAGGAGTTAACGTACGCCGACATC 
TCGCGCCTTCCACTCCTCAATCAAGTTGTCAAGGAGACACTCCGCCTTCATGCTCCCATC CATTCTATTCTGCGACAAGTCAAGTCTCCGATGCCACTCGAAGGTACACCATACGTTGTC CCGACCACACACTCCCTCCTTGCTGCACCCGGTGCTACCTCACGAATGGACGAGCACTTC CCCGAAGCTATGCTGTGGGAACCCCACCGATGGGACGAGAACCCAAGTGAGAAGTACGCA CATCTCGCACCAAAGCATGTCAAGGAGGGCGTCGCCGAAGAGACTGAAGATTACGGCAAT GGTCTCGTCAGCAAAGGCGCCGCATCACCATATCTGCCATTCGGTGCTGGCCGACATAGA TGCATCGGCGAGCAATTCGCCTATGTCCAGCTCCAGACCATCACCTCGGAAGTGATTCGC GATTTCAAGCTCTACAATGTCGACGGCAGCGACAAAGTTGTCGGCACAGATTACAGTTCG TTGTTCAGCAGACCTCTCTCGCCAGCCGTCGTGCGATGGGAGAGGAGAGAAAGAAATA GATTTCACAGTA

>X845-Consensus

GCAGGTGCAAGGTGCGGCGTGATGGTTGGACCCTTGGACCCTCGCAATTGA

TGAGAAGCAGGGGTGTCCCGTTCCTGCATGACTAGCGCCAAAAAGTGACGCGACTGTCGA AATGTTAAATCTCGTACGATTGCACCTGCCATGGACCAC

TCGAACAACACTGAAGGGTAATCATTCAAGACCTTGGTGATTTGGCCTAACCCCC GACACGAACATCAACATCACTGACACCGCGCAATGGGGCTCCTCCAAGACGCTGCGGCGC TTTTCGACGCGCAATTTGGCCAGATAGCGACATGGAAACTTGTCCCCCTCGGCTTCAGCA TCTTCTTCGCCGTATCCGTGTTGCTTAACGTGTTGCGCCAGCTGCTCTTCAGAAATCCAA ACGAACCTCCGCTAGTATTCCACTTCGTGCCCTTCATTGGCAGCACTATCTCCTATGGCA TCGACCCCTACAAGTTCTTCTTCGCCTGCCGTCAAAAATACGGAGATTGCTTCACTTTCA TCCTCCTTGGCAAGAAGACCACCGTGGTGCTGGGGACTAAAGGCAACGACTTTATCTTGA ATGGAAAGCTCAAGGACGTCAATGCCGAGGAGATCTATAGCCCACTTACTACGCCAGTAT TCGGCACAGATGTCGTCTACGATTGTCCCAATTCGAAGCTCATGGAGCAGAAGAAGGTGC GTGCACACAATAAGCTGGATTTATGACAATGCTAACTTATACAGTTCGTCAAATATGGTC AGTGACTTGACTCCGCCGCGTCTGAGACAGCGCTCTCAACATGAGCTTCCACTTTGGAGC GATACACTTGCTAACCTGCGCCTCCAGGCCTCACCTCCTCCGCCCTCCAGTCCTACGTTA AATTGATCACCAAAGAGACCAAAGACTTCTTCTCCAAGGACAATCCAAGCAAGAAATTCG CATCCACCCACGGCACCGTCGACCTCCCGCCTGCTATGGCTGAGCTTACTATCTACACCG CCAGCCGTTCGCTCCAGGGCAAAGAAGTCCGCGAAAAATTCGACTCCTCCTTTGCCGACC TCTACCACGATCTCGACATGGGCTTCACTCCCATCAACTTCATGCTTCCATGGGCTCCAC TGCCACAGAA-TAGAGCACGCGATCGCGCACAGAAGAAGATGGCGGAAGTCTACACAGCG ATCATCAAAGAGCGACGCGAAAAGGGCGAGCCTACTTCGGGAGAGAAAGAGCAGGACATG ATTTGGAATCTGATGCAATGTCAGTACAAGAATGGTCAAGCAATTCCAGATAAGGAGATT GCGCACATGATGATTGCCCTTCTCATGGCTGGTCAACACTCGTCCTCGTCCACCTCATGC TGGATCCTTCTCCGACTGGCTTCGCGACCAGATATCCAGGATGAGCTCCTTCAAGAACAG AAGGATGTGCTTGGCGTCAACGCAGATGGATCAATCAAGGAGTTAACGTACGCCGACATC TCGCGCCTTCCACTCCTCAATCAAGTTGTCAAGGAGACACTCCGCCTTCATGCTCCCATC CATTCTATTCTGCGACAAGTCAAGTCTCCGATGCCACTCGAAGGTACACCATACGTTGTC CCGACCACACACTCCCTCCTTGCTGCACCCGGTGCTACCTCACGAATGGACGAGCACTTC CCCGAAGCTATGCTGTGGGAACCCCACCGATGGGACGAGAACCCAAGTGAGAAGTACGCA CATCTCGCACCAAAGCATGTCAAGGAGGGCGTCGCCGAAGAGACTGAAGATTACGGCTAT GGTCTCGTCAGCAAAGGCGCCGCATCACCATATCTGCCATTCGGTGCTGGCCGACATAGA TGCATCGGCGAGCAATTCGCCTATGTCCAGCTCCAGACCATCACCTCGGAAGTGATTCGC GATTTCAAGCTCTACAATGTCGACGGCAGCGACAAAGTTGTCGGCACAGATTACAGTTCG TTGTTCAGCAGACCTCTCTCGCCAGCCGTCGTGCGAT-GGGAGAGGAGAGAAAAGAAATA GATTTCACAGTAAGA

\section{$>$ X846-Consensus}

CGAGATGGCGCAGGTGCAAGGTGCGGCGTGATGGTTGGACCCTTGGACCCTCGCAATTGA TGAGAAGCAGGGGTGTCCCGTTCCTGCATGACTAGCGCCAAAAAGTGACGCGACTGCCGA AATGTTAAATCTCGTACGATAGCACCTGCCCATGGACCAC

TCGAACATCACTGAAGGGTAATCATTCAAGATCTTGGTGATTTGGCCTAACCCCC

TACACCAACATCAACATCACTGACTCCGCGCAATGGGGCTCCTCCAGGACGCTGCGGCGC TTTTCGACGCGCAATTTGGCCAGATAGCGACATGGAAACTTGTCCCCCTCGGCTTCAGCA TCTTCTTCGCCGTATCCGTGTTGCTTAACGTGTTGCGCCAGCTGCTCTTCAGAAATCCAA ACGAACCTCCGCTAGTATTCCACTACGTGCCCTTCATTGGCAGCACTATCTCCTATGGCA TCGACCCCTACAAGTTCTTCTTCGCCTGCCGTCAAAAATACGGAGATTGCTTCACTTTCA TCCTCCTTGGCAAGAAGACCACCGTGGTGCTGGGGACTAAAGGCAACGACTTTATCTTGA ATGGAAAGCTCAAGGACCTCAATGCCGAGGAGATCTATAGCCCACTTACTACCCCAGTAT TCGGCACAGATGTCGTCTACGATTGTCCCAATTCGAAGCTCATGGAGCAGAAGAAGGTGC GTGCACACAATAAGCTGGGTTTATGACAATGCTAACTTATACAGTTCGTCAAATATGGTC AGTGACTTGACTCCGCCGCGTCTGAGACAGCGCTCTGAACATGAGCTTCCACTTTGGAGC GATACACTTGCTAACCTCCGCCTCCAGGCCTCACCTCCTCCGCCCTCCAGTCCTACGTTA AATTGATCACCAAAGAGACCAAAGACTTCTTCTCCAAGGACAATCCAAGCAAGAAATTCG CATCCACCCACGGCACCGTCGACCTCCCGCCTGCTATGGCTGAGCTTACTATCTACACCG CCAGCCGTTCGCTCCAGGGCAAAGAAGTCCGCGAAAAATTCGACTCCTCCTTTGCCGACC 


\section{CHAPTER 4}

TCTACCACGATCTCGACATGGGCTTCACTCCCATCAACTTCATGCTTCCATGGGCTCCAC TGCCACAGAA-TAGAGCACGCGATCGCGCACAGAAGAAGATGGCGGAAGTCTACACAGCG ATCATCAAAGAGCGACGCGAAAAGGGCGAGCCTACTTCGGGAGAGAAAGAGCAGGACATG ATTTGGAATCTGATGCAATGTCAGTACAAGAATGGTCAAGCAATTCCAGATAAGGAGATT GCGCACATGATGATTGCCCTTCTCATGGCTGGTCAACACTCGTCCTCGTCCACCTCATGC TGGATCCTTCTCCGACTGGCTTCGCGACCAGATATCCAGGATGAGCTCCTTCAAGAACAG AAGGATGTGCTTGGCGTCAACGCAGATGGATCAATCAAGGAGTTAACGTACGCCGACATC TCGCGCCTTCCACTCCTCAATCAAGTTGTCAAGGAGACACTCCGCCTTCATGCTCCCATC CATTCTATTCTGCGACAAGTCAAGTCTCCGATGCCACTCGAAGGTACACCATACGTTGTC CCGACCACACACTCCCTCCTTGCTGCACCCGGTGCTACCTCACGAATGGACGAGCACTTC CCCGAAGCTATGCTGTGGGAACCCCACCGATGGGACGAGAACCCAAGTGAGAAGTACGCA CATCTCGCACCAAAGCATGTCAAGGAGGGCGTCGCCGAAGAGACTGAAGATTACGGCTAT GGTCTCGTCAGCAAAGGCGCCGCATCACCATATCTGCCATTCGGTGCTGGCCGACATAGA TGCATCGGCGAGCAATTCGCCTATGTCCAGCTCCAGACCATCACCTCGGAAGTGATTCGC GATTTCAAGCTCTACAATGTCGACGGCAGCGACAAAGTTGTCGGCACAGATTACAGTTCG TTGTTCAGCAGACCTCTCTCGCCAGCCGTCGTGCGATGGGAGAGGAGAGAAAGAAATA GATTTCACAGTAAGA

$>$ >x847_Consensus GGTGCAGATGCAAGGTGCGGCGTGATGGTTGGACCCTTGGACCCTCGCAATTGA TGAGAAGCAGGGGTGTCCCGTTCCTGCATGACTAGCGCCAAAAAGTGACGCGACTGCCGA AATGTTAAATCTCGTACGATAGCACCTGCCCATGGACCAC

TCGAACCACACTGAAGGGTAATCATTCAAGATCTTGGTGATTTGGCCTAACTTCC TACACCAACATCAACATCACTGACTCCGCGCAATGGGGCTCCTCCAGGACGCCGCGGCGC TTTTCGACGCGCAATTTGGCCAGACAGCGACATGGAAACTAGTCCCCCTCGGCTTCAGCA TCTTCTTCGCCGTATCCGTGTTGCTTAACGTGTTGCGCCAGCTGCTCTTCAGAAATCCGA ACGAACCTCCGCTAGTATTCCACTACGTGCCCTTCATTGGCAGCACTATCTCCTATGGCA TCGACCCCTACAAGTTCTTCTTCGCCTGCCGTCAAAAATACGGAGATTGCTTCACTTTCA TCCTCCTTGGCAAGAAGACCACCGTGGTGCTGGGGACTAAAGGCAACGACTTCATCTTGA ATGGAAAGCTCAAGGACGTCAATGCCGAGGAGATCTATAGCCCACTTACTACGCCAGTAT TCGGCACAGATGTCGTCTACGATTGTCCCAATTCGAAGCTCATGGAGCAGAAGAAGGTGC GTGCACACAATAAGCTGGATTTATGACAATGCTAACTTATACAGTTCGTCAAATATGGTC AGTGACTTGACTCCGCCGCGTCTGAGACAGCGATCTGAACATGAGCTTCCACTTTGGAGC GATACACTTGCTAACCTCCGCCTCCAGGCCTCACCTCCTCCGCCCTCCAGTCCTACGTTA AATTGATCACCAAAGAGACCAGAGACTTCTTCTCCAAGGACAATCCAAGCAAGAAATTCG CATCCACCCACGGCACCGTCGACCTCCCGCCTGCTATGGCTGAGCTTACTATCTACACCG CCAGCCGTTCGCTCCAGGGCAAAGAAGTCCGCGAAAAATTCGACTCCTCCTTTGCCGACC TCTACCACGATCTCGACATGGGCTTCACTCCCATCAACTTCATGCTTCCATGGGCTCCAC TGCCACAGAATAGAGCACGCGATCGCGCACAGAAGAAGATGGCGGAAGTCTACACAGCG ATCATCAAAGAGCGACGCGAAAAGGGCGAGCCTACTTCGGGAGAGAAAGAGCAGGACATG ATTTGGAATCTGATGCAATGTCAGTACAAGAATGGTCAAGCAATTCCAGATAAGGAGATT GCGCACATGATGATTGCCCTTCTCATGGCTGGTCAACACTCGTCTTCGTCCACCTCATGC TGGATCCTTCTCCGACTGGCTTCGCGACCAGATATCCAGGATGAGCTCCTTCAAGAACAG AAGGATGTGCTTGGCGTCAACGCAGATGGATCAATCAAGGAGTTAACGTACGCCGACATC TCGCGCCTTCCACTCCTCAATCAAGTTGTCAAGGAGACACTCCGCCTTCATGCTCCCATC CATTCTATTCTGCGACAAGTCAAGTCTCCGATGCCACTCGAAGGTACACCATACGTTGTC CCGACCACACACTCCCTCCTTGCTGCACCCGGTGCTACCTCACGAATGGACGAGCACTTC CCCGAAGCTATGCTGTGGGAACCCCACCGATGGGACGAGAACCCAAGTGAGAAGTACGCA CATCTCTCACCAAAGCATGTCAAGGAGGGCGTCGCCGAAGAGACTGAAGATTACGGCTAT GGTCTCGTCAGCAAAGGCGCCGCATCACCATATCTGCCATTCGGTGCTGGCCGACATAGA TGCATCGGCGAGCAATTCGCCTATGTCCAGCTCCAGACCATCACCTCAGAAGTGATTCGC GATTTCAAGCTCTACAATGTCGACGGCAGCGACAAAGTTGTCGGCACAGATTACAGTTCG TTGTTCAGCAGACCTCTCTCGCCAGCCGTCGTGCGATGGGAGAGGAGAGAAAGAAATA GATTTCACAGTAGAGTAT

$>$ x849 Consensus TTGGACCCTTGGACCCTCGCAATTGA TGAGAAGCAGGGGTGTCCCGTTCCTGCATGACTAGCGCCAAAAAGTGACGCGACTGCCGA AATGTTAAATCTCGTACGATAGCACCTGCCCATGGACCAC TCGAACATCACTGAAGGGTAATCATTCAAGATCTTGGTGATTTGGCCTAACCCCC TACACCAACATCAACATCACTGACTCCGCGCAATGGGGCTCCTCCAGGACGCCGCGGCGC TTTTCGACGCGCAATTTGGCCAGACAGCGACATGGAAACTAGTCCCCCTCGGCTTCAGCA TCTTCTTCGCCGTATCCGTGTTGCTTAACGTGTTGCGCCAGCTGCTCTTCAGAAATCCAA ACGAACCTCCGCTAGTATTCCACTACGTGCCCTTCATTGGCAGCACTATCTCCTATGGCA TCGACCCCTACAAGTTCTTCTTCGCCTGCCGTCAAAAATACGGAGATTGCTTCACTTTCA TCCTCCTTGGCAAGAAGACCACCGTGGTGCTGGGGACTAAAGGCAACGACTTTATCTTGA ATGGAAAGCTCAAGGACGTCAATGCCGAGGAGATCTATAGCCCACTTACTACGCCAGTAT TCGGCACAGATGTCGTCTACGATTGTCCCAATTCGAAGCTCATGGAGCAGAAGAAGGTGC 
GTGCACACAATAAGCTGGATTTATGACAATGCTAACTTATACAGTTCGTCAAATATGGTC AGTGACTTGACTCCGCCGCGTCTGAGACAGCGCTCTCAACATGAGCTTCCACTTTGGAGC GATACACTTGCTAACCTGCGCCTCCAGGCCTCACCTCCTCCGCCCTCCAGTCCTACGTTA AATTGATCACCAAAGAGACCAAAGACTTCTTCTCCAAGGACAATCCAAGCAAGAAATTCG CATCCACCCACGGCACCGTCGACCTCCCGCCTGCTATGGCTGAGCTTACTATCTACACCG CCAGCCGTTCGCTCCAGGGCAAAGAAGTCCGCGAAAAATTCGACTCCTCCTTTGCCGACC TCTACCACGATCTCGACATGGGCTTCACTCCCATCAACTTCATGCTTCCATGGGCTCCAC TGCCACAGAATAGAGCACGCGATCGCGCACAGAAGAAGATGGCGGAAGTCTACACAGCG ATCATCAAAGAGCGACGCGAAAAGGGCGAGCCTACTTCGGGAGAGAAAGAGCAGGACATG ATTTGGAATCTGATGCAATGTCAGTACAAGAATGGTCAAGCAATTCCAGATAAGGAGATT GCGCACATGATGATTGCCCTTCTCATGGCTGGTCAACACTCGTCCTCGTCCACCTCATGC TGGATCCTTCTCCGACTGGCTTCGCGACCAGATATCCAGGATGAGCTCCTTCAAGAACAG AAGGATGTGCTTGGCGTCAACGCAGATGGATCAATCAAGGAGTTAACGTACGCCGACATC TCGCGCCTTCCACTCCTCAATCAAGTTGTCAAGGAGACACTCCGCCTTCATGCTCCCATC CATTCTATTCTGCGACAAGTCAAGTCTCCGATGCCACTCGAAGGTACACCATACGTTGTC CCGACCACACACTCCCTCCTTGCTGCACCCGGTGCTACCTCACGAATGGACGAGCACTTC CCCGAAGCTATGCTGTGGGAACCCCACCGATGGGACGAGAACCCAAGTGAGAAGTACGCA CATCTCGCACCAAAGCATGTCAAGGAGGGCGTCGCCGAAGAGACTGAAGATTACGGCTAT GGTCTCGTCAGCAAAGGCGCCGCATCACCATATCTGCCATTCGGTGCTGGCCGACATAGA TGCATCGGCGAGCAATTCGCCTATGTCCAGCTCCAGACCATCACCTCGGAAGTGATTCGC GATTTCAAGCTCTACAATGTCGACGGCAGCGACAAAGTTGTCGGCACAGATTACAGTTCG TTGTTCAGCAGACCTCTCTCGCCAGCCGTCGTGCGATGGGAGAGGAGAGAAAAGAAATA GATTTCACAGTAA

>ZTSC_77_Consensus ATGGTGCAGATGATGGTTGGACCCTTG-GACCCTCGCAATTGA

TGAGAAGCAGGGGTGTCCCGTTCCTGCATGACTAGCGCCAAAAAGTGACGCGAATGCCGA AATGTTAAATCTCGTACGATAGCACCTGCCCATGGACCACTCGAACCACACTGAAGGGTAATCATTCAAGATCTTGGTGATTTGGCCTAACCCCC TACACCAACATCAACATCACTGACTCCGCGCAATGGGACTCCTCCAGGACGCCGCGGCGC TTTTCGACGCGCAATTTGGCCAGATAGCGACATGGAAACTAGTCCCCCTCGGCTTCAGCA TCTTCTTCGCCGTATCCGTGTTGCTTAACGTGTTGCGCCAGCTGCTTTTTCAGAAATCCAA ACGAACCTCCGCTAGTATTCCACTACGTGCCCTTCATTGGCAGCACTATCTCCTATGGCA TCGACCCCTACAAGTTCTTCTTCGCCTGCCGTCAAAAATACGGAGATTGCTTCACTTTCA TCCTCCTTGGCAAGAAGACCACCGTGGTGCTGGGGACTAAAGGCAACGACTTTATCTTGA ATGGAAAGCTCAAGGACGTCAATGCCGAGGAGATCTATAGCCCACTTACTACGCCAGTAT TCGGCACAGATGTCGTCTACGATTGTCCCAATTCGAAGCTCATGGAGCAGAAGAAGGTGC GTGCACACAATAAGCTGGATTTATGACAATGCTAACTTATACAGTTCGTCAAATATGGTC AGTGACTTGACTCCGCCGCGTCTGAGACAGCGCTCTCAACATGAGCTTCCACTTTGGAGC GATACACTTGCTAACCTGCGCCTCCAGGCCTCACCTCCTCCGCCCTCCAGTCCTACGTTA AATTGATCACCAAAGAGACCAAAGACTTCTTCTCCAAGGACAATCCAAGCAAGAAATTCG CATCCACCCACGGCACCGTCGACCTCCCGCCTGCTATGGCTGAGCTTACTATCTACACCG CCAGCCGTTCGCTCCAGGGCAAAGAAGTCCGCGAAAAATTCGACTCCTCCTTTGCCGACC TCTACCACGATCTCGACATGGGCTTCACTCCCATCAACTTCATGCTTCCATGGGCTCCAC TGCCACAGAATAGAGCACGCGATCGCGCACAGAAGAAGATGGCGGAAGTCTACACAGCG ATCATCAAAGAGCGACGCGAAAAGGGCGAGCCTACTTCGGGAGAGAAAGAGCAGGACATG ATTTGGAATCTGATGCAATGTCAGTACAAGAATGGTCAAGCAATTCCAGATAAGGAGATT GCGCACATGATGATTGCCCTTCTCATGGGTGGTCAACACTCGTCCTCGTCCACCTCATGC TGGATCCTTCTCCGACTGGCTTCGCGACCAGATATCCAGGATGAGCTCCTTCAAGAACAG AAGGATGTGCTTGGCGTCAACGCAGATGGATCAATCAAGGAGTTAACGTACGCCGACATC TCGCGCCTTCCACTCCTCAATCAAGTTGTCAAGGAGACACTCCGCCTTCATGCTCCCATC CATTCTATTCTGCGACAAGTCAAGTCTCCGATGCCACTCGAAGGTACACCATACGTTGTC CCGACCACACACTCCCTCCTTGCTGCACCCGGTGCTACCTCACGAATGGACGAGCACTTC CCCGAAGCTATGCTGTGGGAACCCCACCGATGGGACGAGAACCCAAGTGAGAAGTACGCA CATCTCGCACCAAAGCATGTCAAGGAGGGCGTCGCCGAAGAGACTGAAGATTACGGCTCT GGTCTCGTCAGCAAAGGCGCCGCATCACCATATCTGCCATTCGGTGCTGGCCGACATAGA TGCATCGGCGAGCAATTCGCCTATGTCCAGCTCCAGACCATCACCTCGGAAGTGATTCGC GATTTCAAGCTCTACAATGTCGACGGCAGCGACAAAGTTGTCGGCACAGATTACAGTTCG TTGTTCAGCAGACCTCTCTCGCCAGCCGTCGTGCGATGGGAGAGGAGAGAAAAGAAATA GATTTCACAGTA

>ZTSC 79_Consensus

TGGTGCAGATGATGGTTGGACCCTTGGACCCTCGCAATTG

ATGAGAAGCAGGGGTGTGCGGTCCTGCATGACTAGCGCCAAAAAGTGACGCGAATGCCGA AATGTTAAATCTCGTACGATAGCACCTGCCCATGGACCAC

TCGAACCACACTGAAGGGTAATCATTCAAGATCTTGGTGATTTGGCCTAACCCCC

TACACCAACATCAACATCACTGACTCCGCGCAATGGGACTCCTCCAGGACGCCGCGGCGC TTTTCGACGCGCAATTTGGCCAGATAGCGACATGGAAACTAGTCCCCCTCGGCTTCAGCA 


\section{CHAPTER 4}

TCTTCTTCGCCGTATCCGTGTTGCTTAACGTGTTGCGCCAGCTGCTTTTCAGAAATCCAA ACGAACCTCCGCTAGTATTCCACTACGTGCCCTTCATTGGCAGCACTATCTCCTATGGCA TCGACCCCTACAAGTTCTTCTTCGCCTGCCGTCAAAAATACGGAGATTGCTTCACTTTCA TCCTCCTTGGCAAGAAGACCACCGTGGTGCTGGGGACTAAAGGCAACGACTTTATCTTGA ATGGAAAGCTCAAGGACGTCAATGCCGAGGAGATCTATAGCCCACTTACTACGCCAGTAT TCGGCACAGATGTCGTCTACGATTGTCCCAATTCGAAGCTCATGGAGCAGAAGAAGGTGC GTGCACACAATAAGCTGGATTTATGACAATGCTAACTTATACAGTTCGTCAAATATGGTC AGTGACTTGACTCCGCCGCGTCTGAGACAGCGCTCTCAACATGAGCTTCCACTTTGGAGC GATACACTTGCTAACCTGCGCCTCCAGGCCTCACCTCCTCCGCCCTCCAGTCCTACGTTA AATTGATCACCAAAGAGACCAAAGACTTCTTCTCCAAGGACAATCCAAGCAAGAAATTCG CATCCACCCACGGCACCGTCGACCTCCCGCCTGCTATGGCTGAGCTTACTATCTACACCG CCAGCCGTTCGCTCCAGGGCAAAGAAGTCCGCGAAAAATTCGACTCCTCCTTTGCCGACC TCTACCACGATCTCGACATGGGCTTCACTCCCATCAACTTCATGCTTCCATGGGCTCCAC TGCCACAGAATAGAGCACGCGATCGCGCACAGAAGAAGATGGCGGAAGTCTACACAGCG ATCATCAAAGAGCGACGCGAAAAGGGCGAGCCTACTTCGGGAGAGAAAGAGCAGGACATG ATTTGGAATCTGATGCAATGTCAGTACAAGAATGGTCAAGCAATTCCAGATAAGGAGATT GCGCACATGATGATTGCCCTTCTCATGGCTGGTCAACACTCGTCCTCGTCCACCTCATGC TGGATCCTTCTCCGACTGGCTTCGCGACCAGATATCCAGGATGAGCTCCTTCAAGAACAG AAGGATGTGCTTGGCGTCAACGCAGATGGATCAATCAAGGAGTTAACGTACGCCGACATC TCGCGCCTTCCACTCCTCAATCAAGTTGTCAAGGAGACACTCCGCCTTCATGCTCCCATC CATTCTATTCTGCGACAAGTCAAGTCTCCGATGCCACTCGAAGGTACACCATACGTTGTC CCGACCACACACTCCCTCCTTGCTGCACCCGGTGCTACCTCACGAATGGACGAGCACTTC CCCGAAGCTATGCTGTGGGAACCCCACCGATGGGACGAGAACCCAAGTGAGAAGTACGCA CATCTCGCACCAAAGCATGTCAAGGAGGGCGTCGCCGAAGAGACTGAAGATTACGGCTAT GGTCTCGTCAGCAAAGGCGCCGCATCACCATATCTGCCATTCGGTGCTGGCCGACATAGA TGCATCGGCGAGCAATTCGCCTATGTCCAGCTCCAGACCATCACCTCGGAAGTGATTCGC GATTTCAAGCTCTACAATGTCGACGGCAGCGACAAAGTTGTCGGCACAGATTACAGTTCG TTGTTCAGCAGACCTCTCTCGCCAGCCGTCGTGCGANTGGGAGAGGAGAGAAAAGAAATA GATTTCACAGTAAGA 


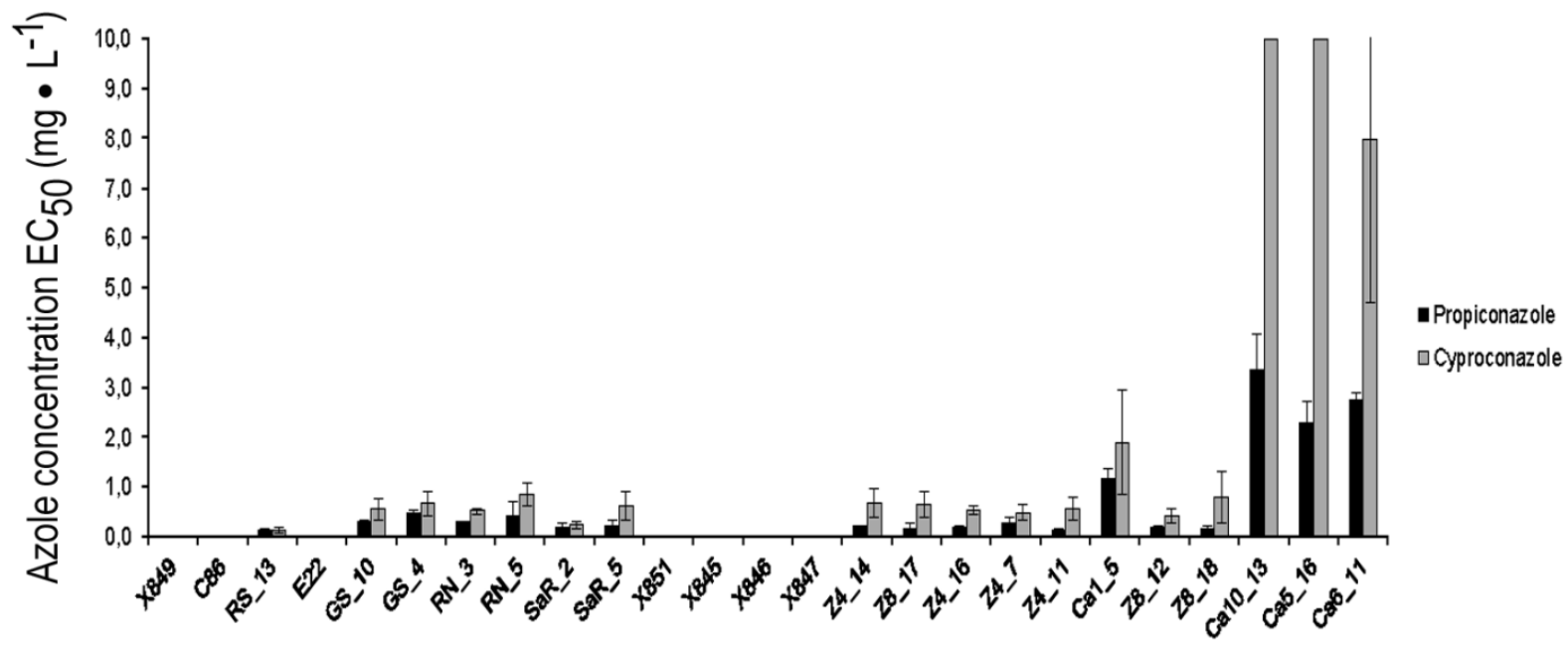

Pseudocercospora fijiensis isolates

Figure S1. Cross-resistance between propiconazole and cyproconazole. The $\mathrm{EC}_{50}$ values were determined for both compounds on Pseudocercospora fijiensis colonies for the indicates strains at 10 days post inoculation (results are means of three independent experiments). 
Table S1. Analysis of Pfcyp51 promoter repeats in 225 Pseudocercospora fijiensis isolates from Costa Rica, compared with 14 isolates from other countries.

\begin{tabular}{|c|c|c|c|c|}
\hline No. & Isolate & Origin & Plantation/Location & Repeat units \\
\hline 1 & $\mathrm{Ca} 1.1$ & Costa Rica & Cartagena & 2 \\
\hline 2 & $\mathrm{Ca} 1.5$ & Costa Rica & Cartagena & 2 \\
\hline 3 & $\mathrm{Ca} 1.7$ & Costa Rica & Cartagena & 6 \\
\hline 4 & $\mathrm{Ca} 1.10$ & Costa Rica & Cartagena & 6 \\
\hline 5 & $\mathrm{Ca} 1.16$ & Costa Rica & Cartagena & 2 \\
\hline 6 & Ca1.20 & Costa Rica & Cartagena & 2 \\
\hline 7 & Ca1.24 & Costa Rica & Cartagena & 6 \\
\hline 8 & $\mathrm{Ca} 2.1$ & Costa Rica & Cartagena & 5 \\
\hline 9 & $\mathrm{Ca} 2.5$ & Costa Rica & Cartagena & 1 \\
\hline 10 & $\mathrm{Ca} 2.11$ & Costa Rica & Cartagena & 1 \\
\hline 11 & $\mathrm{Ca} 2.13$ & Costa Rica & Cartagena & 2 \\
\hline 12 & $\mathrm{Ca} 2.15$ & Costa Rica & Cartagena & 2 \\
\hline 13 & $\mathrm{Ca} 2.16$ & Costa Rica & Cartagena & 2 \\
\hline 14 & $\mathrm{Ca} 2.17$ & Costa Rica & Cartagena & 6 \\
\hline 15 & $\mathrm{Ca} 2.19$ & Costa Rica & Cartagena & 6 \\
\hline 16 & Ca3.1 & Costa Rica & Cartagena & 2 \\
\hline 17 & $\mathrm{Ca} 3.3$ & Costa Rica & Cartagena & 6 \\
\hline 18 & $\mathrm{Ca} 3.5$ & Costa Rica & Cartagena & 6 \\
\hline 19 & Ca3.7 & Costa Rica & Cartagena & 6 \\
\hline 20 & $\mathrm{Ca} 3.10$ & Costa Rica & Cartagena & 2 \\
\hline 21 & $\mathrm{Ca} 3.14$ & Costa Rica & Cartagena & 6 \\
\hline 22 & $\mathrm{Ca} 3.20$ & Costa Rica & Cartagena & 2 \\
\hline 23 & $\mathrm{Ca} 3.22$ & Costa Rica & Cartagena & 6 \\
\hline 24 & $\mathrm{Ca} 3.24$ & Costa Rica & Cartagena & 2 \\
\hline 25 & Ca5.1 & Costa Rica & Cartagena & 6 \\
\hline 26 & $\mathrm{Ca} 5.5$ & Costa Rica & Cartagena & 2 \\
\hline 27 & Ca5.7 & Costa Rica & Cartagena & 6 \\
\hline 28 & $\mathrm{Ca} 5.10$ & Costa Rica & Cartagena & 2 \\
\hline 29 & $\mathrm{Ca} 5.12$ & Costa Rica & Cartagena & 6 \\
\hline 30 & $\mathrm{Ca} 5.13$ & Costa Rica & Cartagena & 6 \\
\hline 31 & Ca5.15 & Costa Rica & Cartagena & 6 \\
\hline 32 & Ca5.16 & Costa Rica & Cartagena & 6 \\
\hline 33 & Ca5.17 & Costa Rica & Cartagena & 6 \\
\hline 34 & Ca5.19 & Costa Rica & Cartagena & 6 \\
\hline 35 & Ca6.1 & Costa Rica & Cartagena & 6 \\
\hline 36 & Ca6.3 & Costa Rica & Cartagena & 6 \\
\hline 37 & $\mathrm{Ca} 6.5$ & Costa Rica & Cartagena & 6 \\
\hline 38 & $\mathrm{Ca} 6.7$ & Costa Rica & Cartagena & 6 \\
\hline 39 & Ca6.9 & Costa Rica & Cartagena & 6 \\
\hline 40 & $\mathrm{Ca} 6.11$ & Costa Rica & Cartagena & 6 \\
\hline 41 & Ca6.12 & Costa Rica & Cartagena & 6 \\
\hline 42 & Ca6.15 & Costa Rica & Cartagena & 6 \\
\hline
\end{tabular}


Table S1. Analysis of Pfcyp51 promoter repeats in 225 Pseudocercospora fijiensis isolates from Costa Rica, compared with 14 isolates from other countries.

\begin{tabular}{|c|c|c|c|c|}
\hline No. & Isolate & Origin & Plantation/Location & Repeat units \\
\hline 43 & Ca6.18 & Costa Rica & Cartagena & 6 \\
\hline 44 & $\mathrm{Ca} 7.1$ & Costa Rica & Cartagena & 2 \\
\hline 45 & $\mathrm{Ca} 7.5$ & Costa Rica & Cartagena & 6 \\
\hline 46 & Ca7.9 & Costa Rica & Cartagena & 2 \\
\hline 47 & $\mathrm{Ca} 7.15$ & Costa Rica & Cartagena & 6 \\
\hline 48 & $\mathrm{Ca} 7.18$ & Costa Rica & Cartagena & 6 \\
\hline 49 & $\mathrm{Ca} 7.20$ & Costa Rica & Cartagena & 2 \\
\hline 50 & $\mathrm{Ca} 7.23$ & Costa Rica & Cartagena & 2 \\
\hline 51 & $\mathrm{Ca} 7.28$ & Costa Rica & Cartagena & 2 \\
\hline 52 & $\mathrm{Ca} 8.2$ & Costa Rica & Cartagena & 6 \\
\hline 53 & $\mathrm{Ca} 8.4$ & Costa Rica & Cartagena & 2 \\
\hline 54 & $\mathrm{Ca} 8.8$ & Costa Rica & Cartagena & 6 \\
\hline 55 & $\mathrm{Ca} 8.11$ & Costa Rica & Cartagena & 6 \\
\hline 56 & $\mathrm{Ca} 8.13$ & Costa Rica & Cartagena & 6 \\
\hline 57 & $\mathrm{Ca} 8.16$ & Costa Rica & Cartagena & 6 \\
\hline 58 & $\mathrm{Ca} 8.20$ & Costa Rica & Cartagena & 6 \\
\hline 59 & $\mathrm{Ca} 8.23$ & Costa Rica & Cartagena & 6 \\
\hline 60 & $\mathrm{Ca} 8.26$ & Costa Rica & Cartagena & 6 \\
\hline 61 & $\mathrm{Ca} 8.28$ & Costa Rica & Cartagena & 2 \\
\hline 62 & $\mathrm{Ca} 8.29$ & Costa Rica & Cartagena & 6 \\
\hline 63 & Ca9.1 & Costa Rica & Cartagena & 6 \\
\hline 64 & $\mathrm{Ca} 9.3$ & Costa Rica & Cartagena & 2 \\
\hline 65 & $\mathrm{Ca} 9.5$ & Costa Rica & Cartagena & 2 \\
\hline 66 & $\mathrm{Ca} 9.8$ & Costa Rica & Cartagena & 2 \\
\hline 67 & Ca9.10 & Costa Rica & Cartagena & 2 \\
\hline 68 & $\mathrm{Ca} 9.12$ & Costa Rica & Cartagena & 2 \\
\hline 69 & Ca9.14 & Costa Rica & Cartagena & 2 \\
\hline 70 & $\mathrm{Ca} 9.17$ & Costa Rica & Cartagena & 2 \\
\hline 71 & Ca9.19 & Costa Rica & Cartagena & 6 \\
\hline 72 & $\mathrm{Ca} 9.22$ & Costa Rica & Cartagena & 2 \\
\hline 73 & Ca10.3 & Costa Rica & Cartagena & 6 \\
\hline 74 & $\mathrm{Ca} 10.5$ & Costa Rica & Cartagena & 6 \\
\hline 75 & Ca10.7 & Costa Rica & Cartagena & 6 \\
\hline 76 & Ca10.10 & Costa Rica & Cartagena & 1 \\
\hline 77 & $\mathrm{Ca} 10.13$ & Costa Rica & Cartagena & 6 \\
\hline 78 & $\mathrm{Ca} 10.23$ & Costa Rica & Cartagena & 6 \\
\hline 79 & $\mathrm{Ca} 10.25$ & Costa Rica & Cartagena & 6 \\
\hline 80 & $\mathrm{Ca} 10.27$ & Costa Rica & Cartagena & 6 \\
\hline 81 & Ca5.18 & Costa Rica & Cartagena & 2 \\
\hline 82 & $\mathrm{Ca} 2.3$ & Costa Rica & Cartagena & 6 \\
\hline 83 & SP1.1 & Costa Rica & San Pablo & 3 \\
\hline 84 & SP1.3 & Costa Rica & San Pablo & 1 \\
\hline
\end{tabular}


Table S1. Analysis of Pfcyp51 promoter repeats in 225 Pseudocercospora fijiensis isolates from Costa Rica, compared with 14 isolates from other countries.

\begin{tabular}{|c|c|c|c|c|}
\hline No. & Isolate & Origin & Plantation/Location & Repeat units \\
\hline 85 & SP1.4 & Costa Rica & San Pablo & 3 \\
\hline 86 & SP1.5 & Costa Rica & San Pablo & 3 \\
\hline 87 & SP1.6 & Costa Rica & San Pablo & 2 \\
\hline 88 & SP1.7 & Costa Rica & San Pablo & 1 \\
\hline 89 & SP1.8 & Costa Rica & San Pablo & 3 \\
\hline 90 & SP2.32 & Costa Rica & San Pablo & 6 \\
\hline 91 & SP3.4 & Costa Rica & San Pablo & 3 \\
\hline 92 & SP3.5 & Costa Rica & San Pablo & 3 \\
\hline 93 & SP3.8 & Costa Rica & San Pablo & 2 \\
\hline 94 & SP5.2 & Costa Rica & San Pablo & 6 \\
\hline 95 & SP5.4 & Costa Rica & San Pablo & 6 \\
\hline 96 & SP5.6 & Costa Rica & San Pablo & 6 \\
\hline 97 & SP5.7 & Costa Rica & San Pablo & 3 \\
\hline 98 & SP5.10 & Costa Rica & San Pablo & 3 \\
\hline 99 & SP5.12 & Costa Rica & San Pablo & 3 \\
\hline 100 & SP5.13 & Costa Rica & San Pablo & 3 \\
\hline 101 & SP5.14 & Costa Rica & San Pablo & 6 \\
\hline 102 & SP5.16 & Costa Rica & San Pablo & 6 \\
\hline 103 & SP6.4 & Costa Rica & San Pablo & 3 \\
\hline 104 & SP6.12 & Costa Rica & San Pablo & 3 \\
\hline 105 & SP7.7 & Costa Rica & San Pablo & 1 \\
\hline 106 & SP7.10 & Costa Rica & San Pablo & 3 \\
\hline 107 & SP7.18 & Costa Rica & San Pablo & 1 \\
\hline 108 & SP7.30 & Costa Rica & San Pablo & 1 \\
\hline 109 & SP8.18 & Costa Rica & San Pablo & 3 \\
\hline 110 & SP8.21 & Costa Rica & San Pablo & 6 \\
\hline 111 & SP8.27 & Costa Rica & San Pablo & 6 \\
\hline 112 & SP9.1 & Costa Rica & San Pablo & 3 \\
\hline 113 & SP9.7 & Costa Rica & San Pablo & 1 \\
\hline 114 & SP9.19 & Costa Rica & San Pablo & 3 \\
\hline 115 & SP9.24 & Costa Rica & San Pablo & 3 \\
\hline 116 & SP10.1 & Costa Rica & San Pablo & 1 \\
\hline 117 & SP10.15 & Costa Rica & San Pablo & 1 \\
\hline 118 & SP10.17 & Costa Rica & San Pablo & 3 \\
\hline 119 & SP6.6 & Costa Rica & San Pablo & 3 \\
\hline 120 & SP6.7 & Costa Rica & San Pablo & 3 \\
\hline 121 & SP6.9 & Costa Rica & San Pablo & 3 \\
\hline 122 & SP6.10 & Costa Rica & San Pablo & 3 \\
\hline 123 & SP6.11 & Costa Rica & San Pablo & 3 \\
\hline 124 & SP6.12 & Costa Rica & San Pablo & 6 \\
\hline 125 & SP7.1 & Costa Rica & San Pablo & 6 \\
\hline 126 & $\mathrm{Z} 1.3$ & Costa Rica & Zent & 1 \\
\hline
\end{tabular}


Table S1. Analysis of Pfcyp51 promoter repeats in 225 Pseudocercospora fijiensis isolates from Costa Rica, compared with 14 isolates from other countries.

\begin{tabular}{|c|c|c|c|c|}
\hline No. & Isolate & Origin & Plantation/Location & Repeat units \\
\hline 127 & Z.1.5 & Costa Rica & Zent & 1 \\
\hline 128 & $\mathrm{Z} 1.8$ & Costa Rica & Zent & 1 \\
\hline 129 & $\mathrm{Z} 1.10$ & Costa Rica & Zent & 1 \\
\hline 130 & $\mathrm{Z} 1.12$ & Costa Rica & Zent & 1 \\
\hline 131 & Z1.14 & Costa Rica & Zent & 1 \\
\hline 132 & $\mathrm{Z} 1.18$ & Costa Rica & Zent & 1 \\
\hline 133 & $\mathrm{Z} 1.20$ & Costa Rica & Zent & 1 \\
\hline 134 & $\mathrm{Z} 1.21$ & Costa Rica & Zent & 1 \\
\hline 135 & $\mathrm{Z} 1.24$ & Costa Rica & Zent & 1 \\
\hline 136 & $\mathrm{Z} 2.1$ & Costa Rica & Zent & 1 \\
\hline 137 & $\mathrm{Z} 2.2$ & Costa Rica & Zent & 1 \\
\hline 138 & $\mathrm{Z} 2.3$ & Costa Rica & Zent & 1 \\
\hline 139 & $\mathrm{Z} 2.5$ & Costa Rica & Zent & 1 \\
\hline 140 & $\mathrm{Z} 2.7$ & Costa Rica & Zent & 1 \\
\hline 141 & $\mathrm{Z} 2.8$ & Costa Rica & Zent & 1 \\
\hline 142 & Z2.9 & Costa Rica & Zent & 1 \\
\hline 143 & $\mathrm{Z} 2.11$ & Costa Rica & Zent & 1 \\
\hline 144 & $\mathrm{Z} 2.13$ & Costa Rica & Zent & 1 \\
\hline 145 & $\mathrm{Z} 2.14$ & Costa Rica & Zent & 1 \\
\hline 146 & $\mathrm{Z} 3.5$ & Costa Rica & Zent & 2 \\
\hline 147 & Z3.6 & Costa Rica & Zent & 2 \\
\hline 148 & Z3.11 & Costa Rica & Zent & 2 \\
\hline 149 & Z3.15 & Costa Rica & Zent & 6 \\
\hline 150 & Z3.17 & Costa Rica & Zent & 6 \\
\hline 151 & $\mathrm{Z} 3.32$ & Costa Rica & Zent & 2 \\
\hline 152 & Z3.34 & Costa Rica & Zent & 6 \\
\hline 153 & $\mathrm{Z} 4.2$ & Costa Rica & Zent & 1 \\
\hline 154 & $\mathrm{Z} 4.7$ & Costa Rica & Zent & 1 \\
\hline 155 & Z4.11 & Costa Rica & Zent & 1 \\
\hline 156 & Z4.12 & Costa Rica & Zent & 1 \\
\hline 157 & Z4.14 & Costa Rica & Zent & 1 \\
\hline 158 & Z4.16 & Costa Rica & Zent & 1 \\
\hline 159 & Z4.17 & Costa Rica & Zent & 1 \\
\hline 160 & Z4.19 & Costa Rica & Zent & 1 \\
\hline 161 & $\mathrm{Z} 4.22$ & Costa Rica & Zent & 1 \\
\hline 162 & Z4.26 & Costa Rica & Zent & 1 \\
\hline 163 & Z4.29 & Costa Rica & Zent & 1 \\
\hline 164 & Z5.1 & Costa Rica & Zent & 6 \\
\hline 165 & Z5.4 & Costa Rica & Zent & 6 \\
\hline 166 & Z5.6 & Costa Rica & Zent & 6 \\
\hline 167 & Z5.12 & Costa Rica & Zent & 1 \\
\hline 168 & $\mathrm{Z} 5.13$ & Costa Rica & Zent & 6 \\
\hline
\end{tabular}


Table S1. Analysis of Pfcyp51 promoter repeats in 225 Pseudocercospora fijiensis isolates from Costa Rica, compared with 14 isolates from other countries.

\begin{tabular}{|c|c|c|c|c|}
\hline No. & Isolate & Origin & Plantation/Location & Repeat units \\
\hline 169 & Z5.15 & Costa Rica & Zent & 6 \\
\hline 170 & Z5.18 & Costa Rica & Zent & 1 \\
\hline 171 & $\mathrm{Z} 5.21$ & Costa Rica & Zent & 1 \\
\hline 172 & $\mathrm{Z} 5.25$ & Costa Rica & Zent & 1 \\
\hline 173 & $\mathrm{Z} 5.32$ & Costa Rica & Zent & 1 \\
\hline 174 & Z6.2 & Costa Rica & Zent & 1 \\
\hline 175 & Z6.3 & Costa Rica & Zent & 1 \\
\hline 176 & Z6.5 & Costa Rica & Zent & 1 \\
\hline 177 & $\mathrm{Z} 6.7$ & Costa Rica & Zent & 1 \\
\hline 178 & Z6.9 & Costa Rica & Zent & 1 \\
\hline 179 & Z6.11 & Costa Rica & Zent & 2 \\
\hline 180 & Z6.13 & Costa Rica & Zent & 1 \\
\hline 181 & Z6.15 & Costa Rica & Zent & 2 \\
\hline 182 & Z6.17 & Costa Rica & Zent & 1 \\
\hline 183 & $\mathrm{Z7.1}$ & Costa Rica & Zent & 1 \\
\hline 184 & $\mathrm{Z7.7}$ & Costa Rica & Zent & 6 \\
\hline 185 & $\mathrm{Z7.9}$ & Costa Rica & Zent & 6 \\
\hline 186 & $\mathrm{Z7.14}$ & Costa Rica & Zent & 1 \\
\hline 187 & $\mathrm{Z7.18}$ & Costa Rica & Zent & 6 \\
\hline 188 & $\mathrm{Z7.28}$ & Costa Rica & Zent & 6 \\
\hline 189 & $\mathrm{Z7.31}$ & Costa Rica & Zent & 6 \\
\hline 190 & Z8.1 & Costa Rica & Zent & 2 \\
\hline 191 & Z8.8 & Costa Rica & Zent & 1 \\
\hline 192 & Z8.11 & Costa Rica & Zent & 1 \\
\hline 193 & Z8.12 & Costa Rica & Zent & 2 \\
\hline 194 & Z8.13 & Costa Rica & Zent & 2 \\
\hline 195 & Z8.17 & Costa Rica & Zent & 1 \\
\hline 196 & Z8.18 & Costa Rica & Zent & 2 \\
\hline 197 & Z8.20 & Costa Rica & Zent & 1 \\
\hline 198 & Z8.25 & Costa Rica & Zent & 1 \\
\hline 199 & $\mathrm{Z} 8.27$ & Costa Rica & Zent & 2 \\
\hline 200 & Z8.35 & Costa Rica & Zent & 1 \\
\hline 201 & Z10.1 & Costa Rica & Zent & 1 \\
\hline 202 & $\mathrm{Z} 10.3$ & Costa Rica & Zent & 1 \\
\hline 203 & Z10.4 & Costa Rica & Zent & 1 \\
\hline 204 & Z10.6 & Costa Rica & Zent & 6 \\
\hline 205 & Z10.7 & Costa Rica & Zent & 1 \\
\hline 206 & $\mathrm{Z} 10.8$ & Costa Rica & Zent & 1 \\
\hline 207 & Z10.9 & Costa Rica & Zent & 1 \\
\hline 208 & Z10.10 & Costa Rica & Zent & 1 \\
\hline 209 & Z10.11 & Costa Rica & Zent & 1 \\
\hline 210 & ZTSC2 & Costa Rica & San Carlos & 1 \\
\hline
\end{tabular}


Table S1. Analysis of Pfcyp51 promoter repeats in 225 Pseudocercospora fijiensis isolates from Costa Rica, compared with 14 isolates from other countries.

\begin{tabular}{|c|c|c|c|c|}
\hline No. & Isolate & Origin & Plantation/Location & Repeat units \\
\hline 211 & ZTSC10 & Costa Rica & San Carlos & 1 \\
\hline 212 & ZTSC15 & Costa Rica & San Carlos & 1 \\
\hline 213 & ZTSC40 & Costa Rica & San Carlos & 1 \\
\hline 214 & ZTSC50 & Costa Rica & San Carlos & 1 \\
\hline 215 & ZTSC55 & Costa Rica & San Carlos & 1 \\
\hline 216 & ZTSC60 & Costa Rica & San Carlos & 1 \\
\hline 217 & ZTSC65 & Costa Rica & San Carlos & 1 \\
\hline 218 & ZTSC75 & Costa Rica & San Carlos & 1 \\
\hline 219 & ZTSC80 & Costa Rica & San Carlos & 1 \\
\hline 220 & ZTSC84 & Costa Rica & San Carlos & 1 \\
\hline 221 & ZTSC90 & Costa Rica & San Carlos & 1 \\
\hline 222 & ZTSC95 & Costa Rica & San Carlos & 1 \\
\hline 223 & ZTSC100 & Costa Rica & San Carlos & 1 \\
\hline 224 & ZTSC101 & Costa Rica & San Carlos & 1 \\
\hline 225 & ZTSC77 & Costa Rica & San Carlos & 1 \\
\hline 226 & E22 & Ecuador & $\mathrm{a}$ & 1 \\
\hline 227 & GS.4 & Ecuador & $\mathrm{a}$ & 1 \\
\hline 228 & GS.10 & Ecuador & $\mathrm{a}$ & 1 \\
\hline 229 & RN.3 & Ecuador & $\mathrm{a}$ & 1 \\
\hline 230 & RN.5 & Ecuador & $\mathrm{a}$ & 1 \\
\hline 231 & RS.13 & Ecuador & $\mathrm{a}$ & 1 \\
\hline 232 & SaR.2 & Ecuador & $\mathrm{a}$ & 1 \\
\hline 233 & SaR.5 & Ecuador & $\mathrm{a}$ & 1 \\
\hline 234 & X845 & Indonesia & $\mathrm{a}$ & 1 \\
\hline 235 & X846 & Phillipines & $\mathrm{a}$ & 1 \\
\hline 236 & X847 & Taiwan & $\mathrm{a}$ & 1 \\
\hline 237 & X849 & Burundi & $\mathrm{a}$ & 1 \\
\hline 238 & X851 & Gabon & $\mathrm{a}$ & 1 \\
\hline 239 & $\mathrm{C} 86$ & Cameroon & $\mathrm{a}$ & 1 \\
\hline
\end{tabular}


CHAPTER 4 


\section{CHAPTER 5}

\section{GENERAL DISCUSSION}


Unraveling a pathosystem is hard work. In this thesis we have begun to scratch the surface of the Sigatoka - banana complex, with a focus on the black Sigatoka pathogen Pseudocercospora fijiensis. The ecological context, however, is that banana encounters the Sigatoka complex, worldwide. Previous analyses unveiled that this complex contains at least 16 fungal species interacting on banana leaves (Arzanlou et al., 2008). We know that $P$. musae has been largely replaced by $P$. fijiensis (Carlier et al., 2000; Zandjanakou-Tachin et al., 2013). Recently, it has been suggested that $P$. eumusae is even more aggressive, but hard data underpinning this suggestion are largely missing. Nevertheless, it is important to realize that $P$. fijiensis is part of a complex ecological reality (Carlier et al., 2000; Chang et al., 2016; Zandjanakou-Tachin et al., 2013). Black Sigatoka is barely a problem in household farming and seems to be the plague of banana plantations, which are among the last remnants of oldfashioned monoculture agriculture. Nearly all export bananas are of the "Cavendish" type, which is susceptible to black Sigatoka (chapter 1). Chong (2016) has shown the consequence of this agricultural model; a highly susceptible globally dispersed host clone leaves virtually only one option for disease management. This is massive usage of fungicides, which leads to an ever-reducing sensitivity to these compounds of the sexually reproducing fungus, eventually leading to unmanageable disease situations. The only way to escape from black Sigatoka is to move, to new less disease prone areas that even allow the production of biological bananas. However, such areas are sparse and usually located in remote regions, at high altitudes where $P$. fijiensis does not thrive due to non-conducive weather conditions. Those areas, however, can also not supply the global demand. Thus, escaping to these new sub-optimal environments is not the way to go. Instead, we need the dive deep and do all the necessary research to (re-)build the system bottom-up, aiming at a radical change in the production systems. This may require expansion of the applied genetic diversity in the field by releasing new banana varieties that meet consumer demands, but also avoiding 
monocultures and invest in multi-cropping by for instance integrating banana production and forestry. As P. fijiensis is light sensitive, similar to many other Dothideomycetes, shading might already be a factor that affects epidemics. Evidently, large monocultures do not provide shade, while agroforestry systems do. Thus, ecological embedding of a host-pathosystem is important and the banana - P. fijiensis pathosystem is not an exception. In this thesis, we have started to unravel the banana $-P$. fijiensis pathosystem as one of the building blocks of a solid foundation for future banana production.

Pseudocercospora fijiensis is a bipolar heterothallic Dothideomycete plant pathogenic fungus (Arzanlou et al., 2010; Conde-Ferráez et al., 2007). It is part of a group of major fungal plant pathogens affecting manifold crops and its life strategy is characterized by abundant sex. These two characteristics are important leads for improved understanding of this pathosystem. In many ways, $P$. fijiensis resembles the wheat foliar blight Zymoseptoria tritici. Twenty-five years ago, the interaction between this fungus and wheat was enigmatic and believed to be merely driven by quantitative variation for resistance - not a single gene for resistance was identified - and pathogenicity. By steady descriptive and explorative research, it is now one of the leading model systems (Brading et al., 2002; Goodwin and Kema, 2014; Kema et al., 2018; Mirzadi Gohari, 2015; Tabib Ghaffary et al., 2011). Many resistance genes (Stb genes) have been identified (Brading et al., 2002; Tabib Ghaffary et al., 2011; Kema et al., 2000) by classical genetics and are being used in commercial breeding programs around the world (Torriani et al., 2015). Recently, the first resistance gene, Stb6, and the first avirulence gene AvrStb6 were cloned (Kema et al., 2018; Saintenac et al., 2018; Zhong et al., 2017). Moreover, Kema et al. (2018) discovered a sexual peculiarity of Z. tritici that most likely applies to many other Dothideomycetes, including $P$. fijiensis; exclusive paternal parenthood (EPP). Incorporation of EPP in epidemiological models explains extended longevity of wheat cultivars as well as the rapid dissemination of strobilurin resistance in $Z$. 
tritici population across Europe. It is very likely that the rapid dissemination and fixation of strobilurin resistance in Costa Rican P. fijiensis populations is also due to EPP (chapter 2; Amil et al., 2007). Hence, sequencing the P. fijiensis genome was the way ahead. It generates interest, attracts researchers, widens the community, involves the industry and leads to a better understanding of genetic diversity. Before publication of the genome sequence (chapter 2), Stergiopoulos et al. $(2010,2014)$ already showed that the effector Pfavr4 is recognized by the tomato Cf4 cognate. We indeed showed that Musa acuminata ssp. burmannicoides var. Calcutta 4, which is iconic for its resistance to black Sigatoka, specifically responded to injections of crude protein extracts of PfAVR4 (chapter 2). This is a first indication that resistance genes in banana for black Sigatoka can be identified and mapped. I speculate that banana contains cognate receptor genes, analogous of $C f 4$. Surprisingly, such studies are limited (Ortiz and Swennen, 2014; Ortiz and Vuylsteke, 1994) and not a single gene for resistance has been identified to $P$. fijiensis. In retrospect, this is explainable as mapping genes by exposing segregating populations to natural $P$. fijiensis populations in the outside environment can only lead to erroneous conclusions. Genetic studies will lead nowhere by placing segregating populations to an anonymous, segregating, highly diverse population of a pathogen. Thus far, one study used individual isolates of $P$. fijiensis on a range of banana accessions (Fullerton and Olsen, 1995). This is analogous to the studies in Z. tritici and an absolute requirement for understanding host-pathogen interactions (Kema et al., 2000; Kema and van Silfhout, 1997; Kema et al., 1996b, 1996a, 2018; Tabib Ghaffary et al., 2011; Ware, 2006). Once P. fijiensis isolates are characterized they can be used individually for genetic analyses. Given the stature of banana plants, such studies require a greenhouse setting, which also precludes any contamination with natural $P$. fijiensis populations. One of the benefits of the $P$. fijiensis genome sequence is that we now can work towards finishing the genome, resequencing other strains and develop an effector portfolio 
from a global population analysis. This contributes to effector-based screening methods and can build on a detailed analysis of the population diversity (Pais et al., 2017). The development of the Agrobacterium-mediated transformation of P. fijiensis is an important achievement allowing functional analysis of important genes. The method was employed to replace the Pfcyp51 promoter to prove that repeat elements in the promoter of this gene contribute to azole fungicides resistance. Further onwards, several results emerging from this thesis will be discussed. Firstly, the implications of the genomic structures such as high repetitive DNA content and transposons on the biology of $P$. fijiensis and its interaction with the host plant. Secondly, how banana breeders can benefit from the hypothesized Pf4 and other resistance genes in banana for $P$. fijiensis resistance. Finally, how the biology of $P$. fijiensis can explain its interaction with and survival under azole fungicide pressure.

\section{The Pseudocercospora fijiensis genome and its biological implications}

We sequenced two $P$. fijiensis strains and assembled the genome by using a genetic linkage map. As discussed above, sexual reproduction is crucial for $P$. fijiensis and hence, it is a great achievement that routine crosses between isolates now can be accomplished under controlled conditions in a greenhouse after identifying the compatible mating types (mat1-1 and mat1-2) of the partners (Chong, 2016). One of the first observations was the massive genetic diversity in natural populations. Electrophoretic karyotyping and analyses of molecular markers in $P$. fijiensis field populations showed chromosome-length polymorphisms and high genetic diversity. Genetic differentiation was also detected using neutral markers, suggesting strong selection with limited gene flow at the studied geographic scale. We studied five samples from the Costa Rican population "Cartagena" (chapter 2), and our data were truly different from the analysis of Mexican strains (Rodríguez-García et al., 2006), which is not surprising due to the high polymorphisms of isolates that was found previously at plant and lesion level (Müller et al., 1997; Rivas et al., 2004). The second 
highlight was the huge genome expansion. Compared to the Dothideomycete reference $Z$. tritici, the $P$. fijiensis genome is, with $74 \mathrm{Mb}$, almost twice as large (Goodwin et al., 2011). The genome contains $49 \%$ repetitive DNA, responsible for the genome expansion, similar to the genomes of the other Sigatoka complex pathogens $P$. musae $(51.5 \mathrm{Mb}, 62.2 \%$ repetitive content and unassembled sequences) and $P$. eumusae $(19.2 \mathrm{Mb}, 35.7 \%$ repetitive content and unassembled sequences) (Chang et al., 2016) and C. fulvum (61.1 Mb, $41 \%$ repetitive content) (de Wit et al., 2012). In P. fijiensis, LTR retrotransposons account for no less than $50 \%$ of the expansion, largely exceeding the $2 \%$ in $Z$. tritici, and other sequenced Dothideomycetes (Dhillon et al., 2014; Goodwin et al., 2011; Ohm et al., 2012) and similar to other unrelated fungal pathogens such as Blumeria graminis f. sp. tritici (180 Mb, 64\% LTRs) (Spanu et al., 2010; Wicker et al., 2013). Until recently, Sigatoka complex pathogens had the largest Dothideomycete genomes, but the current championing genome sizes are those of Cenococcum geophilum, the only ectomycorrhizal symbiont that evolved within the Dothideomycetes, with a genome size of $178 \mathrm{Mb}$ (Peter et al., 2016), and Zopfia rhizophila, an asparagus root rot fungus, with a genome size of $153 \mathrm{Mb}$ (https://genome.jgi.doe.gov/Zoprh1/Zoprh1.home.html). Our melting-curve assays already indicated expanded genomes in P. eumusae and P. musae (chapter 2), which was recently confirmed (Chang et al., 2016). In all these fungi, transposons are the major drivers for genome evolution, similarly to processes in other plant pathogens such as Verticillium dahliae (Faino et al., 2016), Leptosphaeria maculans (Grandaubert et al., 2014) and Pyrenophora tritici repentis (Manning et al., 2013). In many cases, such repeat rich areas, which can be lineage specific, harbor effector genes that are crucial for orchestrating the outcome of hostpathogen interactions, like the effector Pfavr4 on scaffold 4 (Stergiopoulos et al., 2010) . As mentioned above, the banana - P. fijiensis pathosystem is at a very early stage with respect to unravelling specificity. According to the zig-zag model of plant immunity (Jones and Dangl, 
2006), pathogens deliver microbial/pathogen-associated molecular patterns (MAMPs/PAMPs) that after recognition by host receptors elicit PAMP-triggered immunity (PTI). Successful pathogens, however, deliver effectors interfering with PTI and resulting in effector-triggered susceptibility (ETS). Pfavr4 is recognized by NB-LRR proteins, which then activate effectortriggered immunity (ETI), resulting in a hypersensitive response (HR). A preliminary scheme for the P. fijiensis - banana interaction is shown in Figure 1.

A

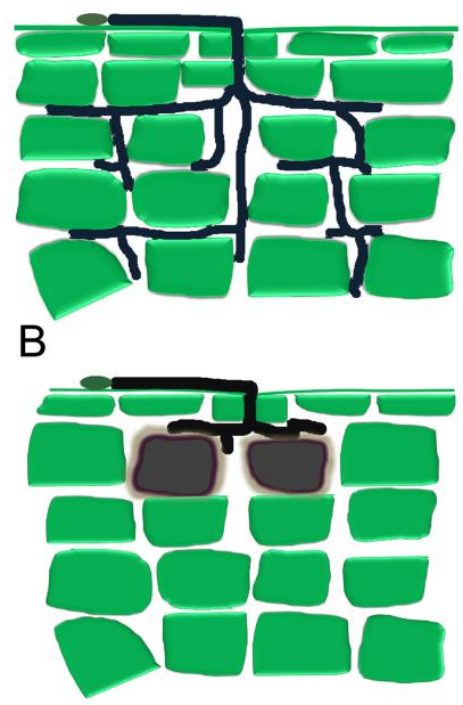

C

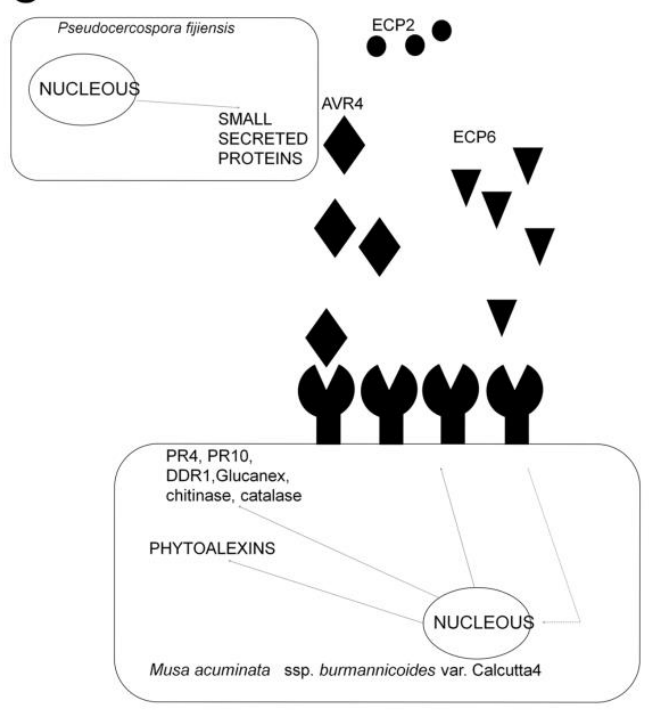

Figure 1. Interaction of Pseudocercospora fijiensis with banana leaf tissue. A) Scheme of a compatible interaction B) Scheme of an incompatible interaction C) Small secreted proteins including PFAVR4, ECP2 and ECP6-like, are expressed by P. fijiensis during the first days of infection and recognized by the resistant accession Musa acuminata ssp. burmannicoides var. Calcutta 4 along with substantial melanin production. The banana host responds with peroxidase production to release reactive oxygen species and starts the production of phytoalexins (Beltrán-García et al., 2014; Cavalcante et al., 2011; Escobar-Tovar et al., 2015; Rodriguez et al., 2016; Torres et al., 2012). At the same time the downstream resistance signaling pathway is initiated after recognition of PFAVR4 by the cognate hypothetical PF4 resistance protein, a process that in turn can be manipulated by ECP6like effector proteins.

Pfavr4 was identified because it was the first effector that showed homology with Avr4 of C. fulvum (Stergiopoulus et al., 2010). In this thesis we showed for the first time that infiltration of the crude Pfavr4 protein in "Calcutta 4" and "Grand Naine" results in a remarkable differential host response. A HR-like response which is characteristic of gene-for- 
gene interactions was observed in "Calcutta 4" but not in "Grand Naine". This suggests that a homologue of the tomato $C f 4$ resistance gene is present in the banana genome. It is therefore likely that we are close to identifying a first black Sigatoka resistance gene. Once such gene has been identified, more effectors and their cognate receptors will be identified, and their frequency can then be monitored in natural populations. Stergiopoulos et al. (2014) initiated such a comparative analysis and concluded that Pfavr4 has at least 17 allelic forms of which some are geographically isolated, suggesting the potential for regional gene deployment as a strategy for disease management. As homologues of Avr4 are present in a number of fungi, it is an interesting gene for comparative functional studies. However, for P. fijiensis the emphasis should lay on identifying more effectors by using bioinformatics on the subset of 172 small secreted proteins ( $<300 \mathrm{bp}$; chapter 2$)$ as well as by classical mapping studies taking the Z. tritici - wheat pathosystem as example (Kema et al., 2018; Mirzadi Gohari, 2015; Zhong et al., 2017) and possibly by using proteomics of infected banana leaves to detect additional putative effectors (Escobar-Tovar et al. 2015; Noar and Daub, 2016b). Finally, proteomic analyses of apoplastic fluids has been a very efficient approach to find effectors of C. fulvum (Bolton et al., 2008; Joosten et al., 1997; Stergiopoulos and de Wit, 2009) and Fusarium oxysporum f. sp. lycopersici (Gawehns et al., 2014; Houterman et al., 2009; Ma et al., 2015). Similar strategies could be used for the banana - P. fijiensis pathosystem, but initial analyses have resulted in practical problems due to the high latex content of apoplastic fluids. Coagulation and oxidation of this latex complicates HPLC analysis. Therefore, the preference lies in generating sequence data to detect more effectors such as in Bipolaris cookie, even without a genome reference (Zaccaron and Bluhm, 2017). Along with precise phenotyping, such data enable genome wide association studies to identify effectors (Zhong et al., 2017). Therefore, developing efficient phenotyping assays should have the highest priority as working with individual $P$. fijiensis isolates is possible, but still rather 
complicated due to the limited experimental amenity of the fungus: it grows slow, has limited conidia production and requires very specific environmental conditions and bio-assays take up to seven weeks. Effector-based phenotyping assays would be ideal for rapid identification of resistance sources, and would be a great support for breeding. Recent studies on the role of proteins such as chitinases and $\beta-1,3$-glucanase (Escobar-Tovar et al., 2015; Portal et al., 2011; Rodriguez et al., 2016; Torres et al., 2012), of peroxidases, and the enzyme phenylalamine ammonia-lyase (PAL), required for the production of precursor phytoalexins (Beltrán-García et al., 2014; Cavalcante et al., 2011; Escobar-Tovar et al., 2015) broaden our view of the interaction, but are beyond the horizon of breeders. This also holds for complicated in vitro assays such as reported by Kovács et al. (2013), who showed that susceptible banana plants transformed with constitutively expressed rice chitinase are resistant to artificially inoculated $P$. fijiensis isolates. First of all, we have to deal with the acceptance of genetically modified crops (Kikulwe et al., 2011; Lucht 2015); and secondly, perform these tests with diverse panel of $P$. fijiensis isolates. Only one isolate was tested, but we now know that $P$. fijiensis has at least 200 effectors and hence the efficacy of resistance has to be tested to the widest possible diversity of virulence factors. Moreover, given the dynamics and diversity of natural $P$. fijiensis populations, it is really questionable whether genetically modified crops are the solution for black Sigatoka in banana (Khanna et al., 2007; Kovács et al., 2013; Mlalazi et al., 2012). The validity of this approach for clonal pathogens such as Fusarium oxysporum f.sp. cubense (Foc) is entirely different. Recently, transgenic "Cavendish" plants harboring a resistance gene from the wild diploid Musa acuminata var. malaccensis were shown to be resistant to the devastating tropical race 4 , which is a great proof of principle (Dale et al., 2017). Moreover, this was the first ever identified resistance gene in banana, despite the fact that resistance of "Cavendish" bananas to race 1 strains of Foc already upholds the industry for decades. Albeit that genetically modified bananas may solve 
a problem in current banana cultivation, the technology does not address the underlying cause and that is genetic uniformity. Hence, particularly with an eye on black Sigatoka, diversification is urgently required and therefore, this study is an important basis for future research into the banana - P. fijiensis pathosystem.

The supposedly rapidly evolving $P$. fijiensis genome could potentially challenge the durability of resistance. TE-rich regions seem instrumental for providing genome plasticity and often harbor effector genes (Raffaele and Kamoun, 2012), as in C. fulvum and D. septosporum (de Wit et al., 2012), Z. tritici (Goodwin et al., 2011), P. nodorum (Hane et al., 2007), M. oryzae (Orbach et al., 2000; Yoshida et al., 2016), and Phytophthora infestans (Raffaele et al., 2010). In L. maculans repeat-induced point mutations (RIP) caused mutations in AvrLml and AvrLm4 to circumvent resistance to Lml and Lm4, respectively (Fudal et al., 2007; Gout et al., 2006; Grandaubert et al., 2014; Rouxel et al., 2011). Also Pfavr4 is next to a transposon that was potentially RIPPed and coincidently showed diversification due to nonsilent mutations (Stergiopoulous et al., 2014). Together with positive selection and intragenic recombination as evolution mechanisms they facilitate evading the banana host immune system. However, until now not a single resistance gene to P. fijiensis was neither identified, nor mapped let alone cloned. Hence, it is important to identify as many resistance genes as possible and to develop the technologies to efficiently accomplish that goal. Recently, a series of resistance genes analogues has been explored (Capdeville et al., 2009; Pei et al., 2007; Sánchez Timm et al., 2016; Wiame et al., 2000), but none could be related to resistance to $P$. fijiensis. Genomic and cDNA libraries of M. acuminata Colla (Cheung and Town, 2007; Vilarinhos et al., 2003) M. acuminata spp. burmannicoides var. Calcutta 4 (Santos et al., 2005), var. TuuGia (Ortiz-Vázquez et al., 2005), var. Pahang (Arango et al., 2011), will also aid gene discovery. However, understanding the biology remains a sound basis for any gene discovery and deployment as exemplified by the aforementioned EPP mechanism, which 
could be operational in the banana - P. fijiensis pathosystem and has a buffering effect of the longevity of resistance (Kema et al., 2018).

The developed ATMT protocol is an important tool for any functional analysis in $P$. fijiensis (chapter 3). The Ku70 protein is essential for the non-homologous end joining (NHEJ) pathway of DNA repair. The generation of a $\triangle P f k u 70$ strain in this study might aid higher homologous recombination rates during transformations. Similarly, CRISPR-Cas9 might be a potential tool for functional analyses in this recalcitrant fungus as it is more precise and less prone to unwanted mutations (Liu et al., 2015). We used ATMT to generate $\triangle P f a v r 4$ strains, which await further characterization, as well as promotor swaps of the cyp51 gene that is the target of the demethylase inhibitor fungicides (DMIs); the work horse of black Sigatoka disease management around the world (chapters 3 and $\mathbf{4}$ ).

\section{Potential of horizontal gene transfer among Pseudocercospora pathogens in the Sigatoka complex}

Mechanisms for horizontal transfer in fungi have been discussed (Manning et al., 2013; Mehrabi et al., 2011, 2017), and the genome of P. fijiensis that is enriched with retrotransposons suggests that it is prone to horizontal gene transfer. $P$. fijiensis, $P$. musae and P. eumusae seem to have a common ancestor and often co-occur in nature (Carlier et al., 2000; Crous and Mourichon, 2002). The genome expansion observed in chapter 2 and by Chang et al. (2016), which is due the high repetitive content in these genomes is suggestive for the potential of horizontal gene transfer. Similar processes were described for the hostselective toxin gene ToxA, which was transferred from Parastagonospora nodorum to Pyrenophora tritici-repentis (Friesen et al., 2006) and the elegant work of Ma et al. (2010) who showed chromosome transfer facilitating pathogenicity to previously non-pathogenic $F$. oxysporum strains on tomato. The Sigatoka complex likely extends beyond the 
aforementioned three species to potentially 16 fungal species (Arzanlou et al., 2008) and therefore provides an excellent system to study the potential and processes involved in horizontal gene transfer. Chang et al. (2016) compared the P. fijiensis genomic data from chapter 2 with the genome sequences of $P$. musae and $P$. eumusae and found reasonably distinct patterns for genome structure and metabolic processes. Goodwin et al. (2011) discerned horizontal gene transfer and RIP degeneration as a potential origin of dispensable chromosomes in Z. tritici, thereby constituting a mechanism for a rapid evolution (Croll et al., 2013). In other fungi such as $N$. haematoccoca supernumerary chromosomes carry virulence genes (Coleman et al., 2009). In M. oryzae, Avr-Pita is located on a dispensable chromosome (Chuma et al., 2011). Therefore these accessory chromosomes may also be part of a genome wide adaptation mechanism. For now, however, we do not know whether the P. fijiensis genome contains accessory chromosomes, but a more detailed analysis would be worthwhile given the physically close proximity of the species in the Sigatoka complex on banana foliage and their potential to engage in such an evolutionary process. It would also cast light on the rapid turn-over of the yellow Sigatoka fungus $P$. musae by the black Sigatoka fungus P.fijiensis, which is much more aggressive and therefore very competitive and still expanding its colonization into new areas (Brito et al., 2015; Rieux et al., 2013). A similar situation could arise with respect to $P$. eumusae, which is now restricted to Southern and Southeast Asia and some areas in Africa, and could compete with P. fijiensis due to other and complementary strategies for host attack (Chang 2016).

\section{Sensitivity of $P$. fijiensis to azoles}

Due to the lack of breeding in banana, despite its importance as a staple food crop and top global fruit commodity, disease control entirely relies on fungicide applications. Chong et al 
(2016) showed that reduced sensitivity in P. fijiensis is a true concern as DMIs are the presently the cornerstone for disease control for black Sigatoka worldwide. As long as there are no commercially viable alternatives, these compounds will remain very important. Therefore, also from this perspective diversification and innovation is required. Fall back scenarios to broad range protective compounds such as chlorothalonil and mineral oils are not the favored strategies for sustainability. It is much better to invest into developing new compounds and alternative products with good efficacy until new resistant varieties reach the markets. In our work we have shown that mutations resulting in amino acid changes in the catalytic domain of CYP51 and promotor insertions affecting gene expression drive reduced sensitivity to DMIs. However, the monitoring of sensitivity in P. fijiensis populations should be professionalized and can be based on our latest data. Quick PCR scans for cyp51 promotor length variation is a first indication of reduced sensitivity and should lead to alternative spraying schedules. Additionally, new products should be evaluated for their efficacy on preventing sexual development. Kema et al. (2018) recently showed that products such as strobilurins, which were very popular and embraced as new active ingredients for black Sigatoka control in banana, do not stop sexual reproduction. This explains why the efficacy of these products rapidly went down once resistant isolates were around (Amil et al., 2007; chapter 2). Finally, black Sigatoka is a burden on the shoulders of those who are interested in growing and consuming organically grown bananas. Without black Sigatoka disease control, producing bananas is virtually impossible unless the entire production zone is moved to new less disease prone areas. Even leaf pruning cannot stop the disease and hence, also this area calls for innovation in the banana production chain. The huge input of fungicides should mobilize consumers to step-up to retailers and all those that dominate the industry. The message should be loud and clear: we want something better than bananas that only survive 
due to environmentally threatening production methods and that are driven only by business as usual strategies. We need truly forward thinking in banana land.

\section{Perspectives}

One of the first things that now need to be performed is developing a reference DNA sequence of $P$. fijiensis. The basis for that analysis is laid in this thesis and a few more rounds of PacBio, or Minion sequencing should be sufficient to finish the genome. This will aid comparative studies across $P$. fijiensis populations and provide a strong support for bioinformatic analyses. One of the first goals would be to determine whether $P$. fijiensis has an accessory set of chromosomes. This is important for all species within the Dothideomycetes, including those with large genomes that carry a highly repetitive DNA (Noar and Daub, 2016b, 2016a). Furthermore, we need to improve the toolbox for P. fijiensis research. The basic ingredients are now available with a sequenced genome (chapter 2), silencing (Onyilo et al., 2017) and transformation protocols (chapter 3). However, these can and will need to be fine-tuned and improved. As mentioned above, harvesting the P. fijiensis genome for effector genes with a strong emphasis on their targets and the development of rapid screening protocols is a necessity to attract new students and scientists into this area and to boost innovation in this orphan crop.

\section{Trending topics}

CRISPR-Cas9 is for many systems the current light at the end of the tunnel. The prospects are enormous and the potential of the technology in nearly any organism is exhilarating (Teboul et al., 2017). The first papers sketch a bright future for banana improvement (Kaur et al., 
2018), but for the screaming disease threats of banana, we first need to identify and map the target genes. From that perspective, banana research is way behind as we do not even know any resistance gene except one (Dale et al., 2017). Hence, the trending topic for future banana research is to focus on the genetics of the interaction between banana and its fungal threats. Without fungicides, western supermarkets will be rapidly depleted from cheap bananas. Hence, all stakeholders of the production and logistic chain need to get together to develop a plan. That plan should envisage a durable and sustainable banana production that supports local economies without jeopardizing the environment and meet consumer demands. Basic science is required and the sector should abandon secretive, repetitive local research aiming at maximizing production. Transparency and critical mass are essential to make progress and hence publishing research is key to lay the foundation for the future. Genomic and genetics will be at the basis for innovative banana improvement and diversity and hence sound production and marketing strategies. I have done my share. Who is next? 


\section{References}

Amil, A.F., Heaney, S.P., Stanger, C., Shaw, M.W., 2007. Dynamics of QoI sensitivity in Mycosphaerella fijiensis in Costa Rica during 2000 to 2003. Phytopathology 97, 1451-1457. https://doi.org/10.1094/PHYTO-97-11-1451

Arango, R.E., Togawa, R.C., Carpentier, S.C., Roux, N., Hekkert, B.L., Kema, G.H.J., Jr, M.T.S., 2011. Genome-wide BAC-end sequencing of Musa acuminata DH Pahang reveals further insights into the genome organization of banana. Tree Genetics \& Genomes 7, $933-$ 940. https://doi.org/10.1007/s11295-011-0385-3

Arzanlou, M., Groenewald, J.Z., Fullerton, R.A., Abeln, E.C.A., Carlier, J., Zapater, M.-F., Buddenhagen, I.W., Viljoen, A., Crous, P.W., 2008. Multiple gene genealogies and phenotypic characters differentiate several novel species of Mycosphaerella and related anamorphs on banana. Persoonia 20, 19-37. https://doi.org/10.3767/003158508X302212

Beltrán-García, M.J., Prado, F.M., Oliveira, M.S., Ortiz-Mendoza, D., Scalfo, A.C., Pessoa, A., Jr, Medeiros, M.H.G., White, J.F., Di Mascio, P., 2014. Singlet Molecular oxygen generation by light-activated dhn-melanin of the fungal pathogen Mycosphaerella fijiensis in black Sigatoka disease of bananas. PLoS ONE 9, e91616. https://doi.org/10.1371/journal.pone.0091616

Bolton, M.D., van Esse, H.P., Vossen, J.H., de Jonge, R., Stergiopoulos, I., Stulemeijer, I.J.E., van den Berg, G.C.M., Borrás-Hidalgo, O., Dekker, H.L., de Koster, C.G., de Wit, P.J.G.M., Joosten, M.H.A.J., Thomma, B.P.H.J., 2008. The novel Cladosporium fulvum lysin motif effector Ecp6 is a virulence factor with orthologues in other fungal species. Mol. Microbiol. 69, 119-136. https://doi.org/10.1111/j.1365-2958.2008.06270.x

Brading, P.A., Verstappen, E.C.P., Kema, G.H.J., Brown, J.K.M., 2002. A gene-for-gene relationship between wheat and Mycosphaerella graminicola, the Septoria tritici blotch pathogen. Phytopathology 92, 439-445. https://doi.org/10.1094/PHYTO.2002.92.4.439

Brito, F.S.D., Fraaije, B., Miller, R.N.G., 2015. Sigatoka disease complex of banana in Brazil: management practices and future directions. Outlooks on Pest Management 26, 78-81. https://doi.org/10.1564/v26_apr_08

Capdeville, G.D., Júnior, M.T.S., Szinay, D., Diniz, L.E.C., Wijnker, E., Swennen, R., Kema, G.H.J., Jong, H.D., 2009. The potential of high-resolution BAC-FISH in banana breeding. Euphytica 166, 431-443. https://doi.org/10.1007/s10681-008-9830-2

Carlier, J., Zapater, M.-F., Lapeyre, F., Jones, D.R., Mourichon, X., 2000. Septoria leaf spot of banana: a newly discovered disease caused by Mycosphaerella eumusae (Anamorph Septoria eumusae). Phytopathology 90, 884-890. https://doi.org/10.1094/PHYTO.2000.90.8.884

Cavalcante, M. de J.B., Escoute, J., Madeira, J.P., Romero, R.E., Nicole, M.R., Oliveira, L.C., Hamelin, C., Lartaud, M., Verdeil, J.L., 2011. Reactive oxygen species and cellular interactions between Mycosphaerella fijiensis and banana. Tropical Plant Biol. 4, 134-143. https://doi.org/10.1007/s12042-011-9071-8

Chang, T.-C., Salvucci, A., Crous, P.W., Stergiopoulos, I., 2016. Comparative genomics of the Sigatoka disease complex on banana suggests a link between parallel evolutionary changes in Pseudocercospora fijiensis and Pseudocercospora eumusae and increased virulence on the banana host. PLoS Genetics In press.

Cheung, F., Town, C.D., 2007. A BAC end view of the Musa acuminata genome. BMC Plant Biology 7, 29. https://doi.org/10.1186/1471-2229-7-29

Chong, P., 2016. Origin, versatility and distribution of azole fungicide resistance in the banana black Sigatoka pathogen Pseudocercospora fijiensis. Wageningen University, Wageningen, the Netherlands.

Chuma, I., Isobe, C., Hotta, Y., Ibaragi, K., Futamata, N., Kusaba, M., Yoshida, K., Terauchi, R., Fujita, Y., Nakayashiki, H., Valent, B., Tosa, Y., 2011. Multiple translocation of the AVR-Pita effector gene among chromosomes of the rice blast fungus Magnaporthe oryzae and related species. PLOS Pathog 7, e1002147. https://doi.org/10.1371/journal.ppat.1002147

Coleman, J.J., Rounsley, S.D., Rodriguez-Carres, M., Kuo, A., Wasmann, C.C., Grimwood, J., Schmutz, J., Taga, M., White, G.J., Zhou, S., Schwartz, D.C., Freitag, M., Ma, L., Danchin, E.G.J., Henrissat, B., Coutinho, P.M., Nelson, D.R., Straney, D., Napoli, C.A., 
Barker, B.M., Gribskov, M., Rep, M., Kroken, S., Molnár, I., Rensing, C., Kennell, J.C., Zamora, J., Farman, M.L., Selker, E.U., Salamov, A., Shapiro, H., Pangilinan, J., Lindquist, E., Lamers, C., Grigoriev, I.V., Geiser, D.M., Covert, S.F., Temporini, E., VanEtten, H.D., 2009. The genome of Nectria haematococca: Contribution of supernumerary chromosomes to gene expansion. PLoS Genet 5, e1000618. https://doi.org/10.1371/journal.pgen.1000618

Croll, D., Zala, M., McDonald, B.A., 2013. Breakage-fusion-bridge cycles and large insertions contribute to the rapid evolution of accessory chromosomes in a fungal pathogen. PLOS Genet 9, e1003567. https://doi.org/10.1371/journal.pgen.1003567

Crous, P.W., Mourichon, X., 2002. Mycosphaerella eumusae and its anamorph Pseudocercospora eumusae spp. nov.: causal agent of eumusae leaf spot disease of banana. Sydowia 54, 35-43.

de Wit, P.J.G.M., van der Burgt, A., Okmen, B., Stergiopoulos, I., Abd-Elsalam, K.A., Aerts, A.L., Bahkali, A.H., Beenen, H.G., Chettri, P., Cox, M.P., Datema, E., de Vries, R.P., Dhillon, B., Ganley, A.R., Griffiths, S.A., Guo, Y., Hamelin, R.C., Henrissat, B., Kabir, M.S., Jashni, M.K., Kema, G., Klaubauf, S., Lapidus, A., Levasseur, A., Lindquist, E., Mehrabi, R., Ohm, R.A., Owen, T.J., Salamov, A., Schwelm, A., Schijlen, E., Sun, H., van den Burg, H.A., van Ham, R.C.H.J., Zhang, S., Goodwin, S.B., Grigoriev, I.V., Collemare, J., Bradshaw, R.E., 2012. The genomes of the fungal plant pathogens Cladosporium fulvum and Dothistroma septosporum reveal adaptation to different hosts and lifestyles but also signatures of common ancestry. PLoS Genet 8. https://doi.org/10.1371/journal.pgen.1003088

Escobar-Tovar, L., Guzmán-Quesada, M., Sandoval-Fernández, J.A., Gómez-Lim, M.A., 2015. Comparative analysis of the in vitro and in planta secretomes from Mycosphaerella fijiensis isolates. Fungal Biology 119, 447-470. https://doi.org/10.1016/j.funbio.2015.01.002

Faino, L., Seidl, M.F., Shi-Kunne, X., Pauper, M., Berg, G.C.M. van den, Wittenberg, A.H.J., Thomma, B.P.H.J., 2016. Transposons passively and actively contribute to evolution of the two-speed genome of a fungal pathogen. Genome Res. https://doi.org/10.1101/gr.204974.116

Friesen, T.L., Stukenbrock, E.H., Liu, Z., Meinhardt, S., Ling, H., Faris, J.D., Rasmussen, J.B., Solomon, P.S., McDonald, B.A., Oliver, R.P., 2006. Emergence of a new disease as a result of interspecific virulence gene transfer. Nat Genet 38, 953-956. https://doi.org/10.1038/ng1839

Fudal, I., Ross, S., Gout, L., Blaise, F., Kuhn, M.L., Eckert, M.R., Cattolico, L., BernardSamain, S., Balesdent, M.H., Rouxel, T., 2007. Heterochromatin-like regions as ecological niches for avirulence genes in the Leptosphaeria maculans genome: map-based cloning of AvrLm6. Mol. Plant Microbe Interact.20, 459-470. https://doi.org/10.1094/MPMI-20-4-0459

Fullerton, R.A., Olsen, T.L., 1995. Pathogenic variability in Mycosphaerella fijiensis Morelet, cause of black Sigatoka in banana and plantain. New Zealand Journal of Crop and Horticultural Science 23, 39-48. https://doi.org/10.1080/01140671.1995.9513866

Gawehns, F., Houterman, P.M., Ichou, F.A., Michielse, C.B., Hijdra, M., Cornelissen, B.J.C., Rep, M., Takken, F.L.W., 2014. The Fusarium oxysporum effector Six6 contributes to virulence and suppresses I-2-mediated cell death. Mol. Plant Microbe Interact. 27, 336-348. https://doi.org/10.1094/MPMI-11-13-0330-R

Goodwin, S.B., Ben M'Barek, S., Dhillon, B., Wittenberg, A.H.J., Crane, C.F., Hane, J.K., Foster, A.J., Van der Lee, T.A.J., Grimwood, J., Aerts, A., Antoniw, J., Bailey, A., Bluhm, B., Bowler, J., Bristow, J., van der Burgt, A., Canto-Canché, B., Churchill, A.C.L., Conde-Ferràez, L., Cools, H.J., Coutinho, P.M., Csukai, M., Dehal, P., De Wit, P., Donzelli, B., van de Geest, H.C., van Ham, R.C.H.J., Hammond-Kosack, K.E., Henrissat, B., Kilian, A., Kobayashi, A.K., Koopmann, E., Kourmpetis, Y., Kuzniar, A., Lindquist, E., Lombard, V., Maliepaard, C., Martins, N., Mehrabi, R., Nap, J.P.H., Ponomarenko, A., Rudd, J.J., Salamov, A., Schmutz, J., Schouten, H.J., Shapiro, H., Stergiopoulos, I., Torriani, S.F.F., Tu, H., de Vries, R.P., Waalwijk, C., Ware, S.B., Wiebenga, A., Zwiers, L.-H., Oliver, R.P., Grigoriev, I.V., Kema, G.H.J., 2011. Finished genome of the fungal wheat pathogen Mycosphaerella graminicola reveals dispensome structure, chromosome plasticity, and stealth pathogenesis. PLoS Genet 7, e1002070. https://doi.org/10.1371/journal.pgen.1002070 
Goodwin, S.B., Kema, G.H.J., 2014. The Genomes of Mycosphaerella graminicola and M. fijiensis, in: Dean, R.A., Lichens-Park, A., Kole, C. (Eds.), Genomics of plant-associated fungi: monocot pathogens. Springer Berlin Heidelberg, pp. 123-140.

Gout, L., Fudal, I., Kuhn, M.-L., Blaise, F., Eckert, M., Cattolico, L., Balesdent, M.-H., Rouxel, T., 2006. Lost in the middle of nowhere: the AvrLm1 avirulence gene of the Dothideomycete Leptosphaeria maculans. Molecular Microbiology 60, 67-80. https://doi.org/10.1111/j.13652958.2006.05076.x

Grandaubert, J., Balesdent, M.-H., Rouxel, T., 2014a. Chapter Three - evolutionary and adaptive role of transposable elements in fungal genomes, in: Martin, F.M. (Ed.), Advances in Botanical Research, Fungi. Academic Press, pp. 79-107.

Grandaubert, J., Lowe, R.G., Soyer, J.L., Schoch, C.L., Wouw, A.P.V. de, Fudal, I., Robbertse, B., Lapalu, N., Links, M.G., Ollivier, B., Linglin, J., Barbe, V., Mangenot, S., Cruaud, C., Borhan, H., Howlett, B.J., Balesdent, M.-H., Rouxel, T., 2014b. Transposable elementassisted evolution and adaptation to host plant within the Leptosphaeria maculansLeptosphaeria biglobosa species complex of fungal pathogens. BMC Genomics 15. https://doi.org/10.1186/1471-2164-15-891

Hane, J.K., Lowe, R.G.T., Solomon, P.S., Tan, K.-C., Schoch, C.L., Spatafora, J.W., Crous, P.W., Kodira, C., Birren, B.W., Galagan, J.E., Torriani, S.F.F., McDonald, B.A., Oliver, R.P., 2007. Dothideomycete plant interactions illuminated by genome sequencing and EST analysis of the wheat pathogen Stagonospora nodorum. Plant Cell 19, 3347-3368. https://doi.org/10.1105/tpc.107.052829

Houterman, P.M., Ma, L., van Ooijen, G., de Vroomen, M.J., Cornelissen, B.J.C., Takken, F.L.W., Rep, M., 2009. The effector protein Avr2 of the xylem-colonizing fungus Fusarium oxysporum activates the tomato resistance protein I-2 intracellularly. Plant J. 58, 970-978. https://doi.org/10.1111/j.1365-313X.2009.03838.x

Jones, J.D.G., Dangl, J.L., 2006. The plant immune system. Nature 444, 323-329. https://doi.org/10.1038/nature05286

Joosten, M.H., Vogelsang, R., Cozijnsen, T.J., Verberne, M.C., De Wit, P.J., 1997. The biotrophic fungus Cladosporium fulvum circumvents Cf-4-mediated resistance by producing unstable AVR4 elicitors. Plant Cell 9, 367-379.

Kaur, N., Alok, A., Shivani, Kaur, N., Pandey, P., Awasthi, P., Tiwari, S., 2018. CRISPR/Cas9mediated efficient editing in phytoene desaturase PDS demonstrates precise manipulation in banana cv. Rasthali genome. Funct Integr Genomics 18, 89-99. https://doi.org/10.1007/s10142-017-0577-5

Kema, G.H., van Silfhout, C.H., 1997. Genetic variation for virulence and resistance in the wheatMycosphaerella graminicola pathosystem III. Comparative seedling and adult plant experiments. Phytopathology 87, 266-272. https://doi.org/10.1094/PHYTO.1997.87.3.266

Kema, G.H., Verstappen, E.C., Waalwijk, C., 2000. Avirulence in the wheat septoria tritici leaf blotch fungus Mycosphaerella graminicola is controlled by a single locus. Mol. Plant Microbe Interact. 13, 1375-1379. https://doi.org/10.1094/MPMI.2000.13.12.1375

Kema, G.H.J., Annone, J.G., Sayoud, R., van Silfhout, C.H., van Ginkel, M., de Bree, J., 1996a. Genetic variation for genetics and virulence in the wheat - Mycosphaerella graminicola pathosystem I. Interactions between pathogen isolates and host cultivars. Phytopathology 86, 200-212.

Kema, G.H.J., Mirzadi Gohari, A., Aouini, L., Gibriel, H.A.Y., Ware, S.B., van den Bosch, F., Manning-Smith, R., Alonso-Chavez, V., Helps, J., Ben M'Barek, S., Mehrabi, R., DiazTrujillo, C., Zamani, E., Schouten, H.J., Van Der Lee, T., Waalwijk, C., De Waard, M.A., De Wit, P.J.G.M., Verstappen, E.C., Thomma, B.P.H.J., Meijer, H.J.G., Seidl, M.F., 2018. Stress and sexual reproduction affect the dynamics of the wheat pathogen effector AvrStb6 and strobilurin resistance | Nature Genetics. In press.

Kema, G.H.J., Yu, D., Rijkenberg, F.H.J., Shaw, M.W., Baayen, R.P., 1996b. Histology of the pathogenesis of Mycosphaerella graminicola in wheat. Phytopathology 86, 777-786.

Khanna, H.K., Paul, J.-Y., Harding, R.M., Dickman, M.B., Dale, J.L., 2007. Inhibition of Agrobacterium-induced cell death by antiapoptotic gene expression leads to very high transformation efficiency of banana. Molecular Plant Microbe Interactions 20, 1048-1054. 
Kikulwe, E.M., Birol, E., Wesseler, J., Falck-Zepeda, J., 2011. A latent class approach to investigating demand for genetically modified banana in Uganda. Agric. Econ. 42, 547-560. https://doi.org/10.1111/j.1574-0862.2010.00529.x

Kovács, G., Sági, L., Jacon, G., Arinaitwe, G., Busogoro, J.-P., Thiry, E., Strosse, H., Swennen, R., Remy, S., 2013. Expression of a rice chitinase gene in transgenic banana ('Gros Michel', AAA genome group) confers resistance to black leaf streak disease. Transgenic Res 22, $117-$ 130. https://doi.org/10.1007/s11248-012-9631-1

Liu, R., Chen, L., Jiang, Y., Zhou, Z., Zou, G., 2015. Efficient genome editing in filamentous fungus Trichoderma reesei using the CRISPR/Cas9 system. Cell Discovery 1. https://doi.org/10.1038/celldisc.2015.7

Lucht, J.M., 2015. Public acceptance of plant biotechnology and GM crops. Viruses 7, 4254-4281. https://doi.org/10.3390/v7082819

Ma, L., Houterman, P.M., Gawehns, F., Cao, L., Sillo, F., Richter, H., Clavijo-Ortiz, M.J., Schmidt, S.M., Boeren, S., Vervoort, J., Cornelissen, B.J.C., Rep, M., Takken, F.L.W., 2015. The AVR2-SIX5 gene pair is required to activate I-2-mediated immunity in tomato. New Phytol. 208, 507-518. https://doi.org/10.1111/nph.13455

Ma, L.-J., van der Does, H.C., Borkovich, K.A., Coleman, J.J., Daboussi, M.-J., Di Pietro, A., Dufresne, M., Freitag, M., Grabherr, M., Henrissat, B., Houterman, P.M., Kang, S., Shim, W.-B., Woloshuk, C., Xie, X., Xu, J.-R., Antoniw, J., Baker, S.E., Bluhm, B.H., Breakspear, A., Brown, D.W., Butchko, R.A.E., Chapman, S., Coulson, R., Coutinho, P.M., Danchin, E.G.J., Diener, A., Gale, L.R., Gardiner, D.M., Goff, S., HammondKosack, K.E., Hilburn, K., Hua-Van, A., Jonkers, W., Kazan, K., Kodira, C.D., Koehrsen, M., Kumar, L., Lee, Y.-H., Li, L., Manners, J.M., Miranda-Saavedra, D., Mukherjee, M., Park, G., Park, J., Park, S.-Y., Proctor, R.H., Regev, A., Ruiz-Roldan, M.C., Sain, D., Sakthikumar, S., Sykes, S., Schwartz, D.C., Turgeon, B.G., Wapinski, I., Yoder, O., Young, S., Zeng, Q., Zhou, S., Galagan, J., Cuomo, C.A., Kistler, H.C., Rep, M., 2010. Comparative genomics reveals mobile pathogenicity chromosomes in Fusarium. Nature 464, 367-373. https://doi.org/10.1038/nature08850

Manning, V.A., Pandelova, I., Dhillon, B., Wilhelm, L.J., Goodwin, S.B., Berlin, A.M., Figueroa, M., Freitag, M., Hane, J.K., Henrissat, B., Holman, W.H., Kodira, C.D., Martin, J., Oliver, R.P., Robbertse, B., Schackwitz, W., Schwartz, D.C., Spatafora, J.W., Turgeon, B.G., Yandava, C., Young, S., Zhou, S., Zeng, Q., Grigoriev, I.V., Ma, L.-J., Ciuffetti, L.M., 2013. Comparative genomics of a plant-pathogenic fungus, Pyrenophora triticirepentis, reveals transduplication and the impact of repeat elements on pathogenicity and population divergence. G3 (Bethesda) 3, 41-63. https://doi.org/10.1534/g3.112.004044

Mehrabi, R., Bahkali, A.H., Abd-Elsalam, K.A., Moslem, M., Ben M'Barek, S., Gohari, A.M., Jashni, M.K., Stergiopoulos, I., Kema, G.H.J., de Wit, P.J.G.M., 2011. Horizontal gene and chromosome transfer in plant pathogenic fungi affecting host range. FEMS Microbiology Reviews 35, 542-554. https://doi.org/10.1111/j.1574-6976.2010.00263.x

Mirzadi Gohari, A., 2015. Identification and functional characterization of putative (a)virulence factors in the fungal wheat pathogen Zymoseptoria tritici. Wageningen University, Wageningen, the Netherlands.

Mlalazi, B., Welsch, R., Namanya, P., Khanna, H.K., Geijskes, R.J., Harrison, M.D., Harding, R.M., Dale, J.L., Bateson, M., 2012. Isolation and functional characterisation of banana phytoene synthase genes as potential cisgenes. Planta.

Müller, R., Pasberg-Gauhl, C., Gauhl, F., Ramser, J., Kahl, G., 1997. Oligonucleotide fingerprinting detects genetic variability at different levels in nigerian Mycosphaerella fijiensis. J Phytopathol 145, 25-30. https://doi.org/10.1111/j.1439-0434.1997.tb00337.x

Noar, R.D., Daub, M.E., 2016a. Transcriptome sequencing of Mycosphaerella fijiensis during association with Musa acuminata reveals candidate pathogenicity genes. BMC Genomics 17, 690. https://doi.org/10.1186/s12864-016-3031-5

Noar, R.D., Daub, M.E., 2016b. Bioinformatics prediction of polyketide synthase gene clusters from $\begin{array}{lllll}\text { Mycosphaerella } \quad \text { fijiensis. } & \text { PLoS } & \text { 11, } & \text { 0158471. }\end{array}$ https://doi.org/10.1371/journal.pone.0158471 
Ohm, R.A., Feau, N., Henrissat, B., Schoch, C.L., Horwitz, B.A., Barry, K.W., Condon, B.J., Copeland, A.C., Dhillon, B., Glaser, F., Hesse, C.N., Kosti, I., LaButti, K., Lindquist, E.A., Lucas, S., Salamov, A.A., Bradshaw, R.E., Ciuffetti, L., Hamelin, R.C., Kema, G.H.J., Lawrence, C., Scott, J.A., Spatafora, J.W., Turgeon, B.G., de Wit, P.J.G.M., Zhong, S., Goodwin, S.B., Grigoriev, I.V., 2012. Diverse lifestyles and strategies of plant pathogenesis encoded in the genomes of eighteen dothideomycetes fungi. PLoS Pathog 8, e1003037. https://doi.org/10.1371/journal.ppat.1003037

Onyilo, F., Tusiime, G., Chen, L.-H., Falk, B., Stergiopoulos, I., Tripathi, J.N., Tushemereirwe, W., Kubiriba, J., Changa, C., Tripathi, L., 2017. Agrobacterium tumefaciens-mediated transformation of Pseudocercospora fijiensis to determine the role of Pfhog1 in osmotic stress regulation and virulence modulation. Front Microbiol 8. https://doi.org/10.3389/fmicb.2017.00830

Orbach, M.J., Farrall, L., Sweigard, J.A., Chumley, F.G., Valent, B., 2000. A telomeric avirulence gene determines efficacy for the rice blast resistance gene Pi-ta. Plant Cell 12, 2019-2032.

Ortiz, R., Vuylsteke, D., 1994. Inheritance of black sigatoka disease resistance in plantain-banana (Musa spp.) hybrids. Theoret. Appl. Genetics 89, 146-152. https://doi.org/10.1007/BF00225134

Ortiz-Vázquez, E., Kaemmer, D., Zhang, H.-B., Muth, J., Rodríguez-Mendiola, M., AriasCastro, C., James, A., 2005. Construction and characterization of a plant transformationcompetent BIBAC library of the black Sigatoka-resistant banana Musa acuminata cv. Tuu Gia (AA). Theor Appl Genet 110, 706-713. https://doi.org/10.1007/s00122-004-1896-1

Pais, M., Yoshida, K., Giannakopoulou, A., Pel, M.A., Cano, L.M., Oliva, R.F., Witek, K., Lindqvist-Kreuze, H., Vleeshouwers, V.G.A.A., Kamoun, S., 2017. Gene expression polymorphism underpins evasion of host immunity in an asexual lineage of the Irish potato famine pathogen. bioRxiv 116012. https://doi.org/10.1101/116012

Pei, X., Li, S., Jiang, Y., Zhang, Y., Wang, Z., Jia, S., 2007. Isolation, characterization and phylogenetic analysis of the resistance gene analogues (RGAs) in banana (Musa spp.). Plant Science 172, 1166-1174. https://doi.org/10.1016/j.plantsci.2007.02.019

Peter, M., Kohler, A., Ohm, R.A., Kuo, A., Krützmann, J., Morin, E., Arend, M., Barry, K.W., Binder, M., Choi, C., Clum, A., Copeland, A., Grisel, N., Haridas, S., Kipfer, T., LaButti, K., Lindquist, E., Lipzen, A., Maire, R., Meier, B., Mihaltcheva, S., Molinier, V., Murat, C., Pöggeler, S., Quandt, C.A., Sperisen, C., Tritt, A., Tisserant, E., Crous, P.W., Henrissat, B., Nehls, U., Egli, S., Spatafora, J.W., Grigoriev, I.V., Martin, F.M., 2016. Ectomycorrhizal ecology is imprinted in the genome of the dominant symbiotic fungus Cenococcum geophilum. Nat Commun 7, 12662. https://doi.org/10.1038/ncomms12662

Portal, O., Izquierdo, Y., Vleesschauwer, D.D., Sánchez-Rodríguez, A., Mendoza-Rodríguez, M., Acosta-Suárez, M., Ocaña, B., Jiménez, E., Höfte, M., 2011. Analysis of expressed sequence tags derived from a compatible Mycosphaerella fijiensis-banana interaction. Plant Cell Rep 30, 913-928. https://doi.org/10.1007/s00299-011-1008-z

Raffaele, S., Kamoun, S., 2012. Genome evolution in filamentous plant pathogens: why bigger can be better. Nature Reviews Microbiology. https://doi.org/10.1038/nrmicro2790

Raffaele, S., Win, J., Cano, L.M., Kamoun, S., 2010. Analyses of genome architecture and gene expression reveal novel candidate virulence factors in the secretome of Phytophthora infestans. BMC Genomics 11, 637. https://doi.org/10.1186/1471-2164-11-637

Rieux, A., De Lapeyre De Bellaire, L., Zapater, M.-F., Ravigne, V., Carlier, J., 2013. Recent range expansion and agricultural landscape heterogeneity have only minimal effect on the spatial genetic structure of the plant pathogenic fungus Mycosphaerella fijiensis. Heredity 110, 29-38. https://doi.org/10.1038/hdy.2012.55

Rivas, G.-G., Zapater, M.-F., Abadie, C., Carlier, J., 2004. Founder effects and stochastic dispersal at the continental scale of the fungal pathogen of bananas Mycosphaerella fijiensis. Mol Ecol 13, 471-482. https://doi.org/10.1046/j.1365-294X.2003.02043.x

Rodriguez, H.A., Rodriguez-Arango, E., Morales, J.G., Kema, G., Arango, R.E., 2016. Defense gene expression associated with biotrophic phase of Mycosphaerella fijiensis M. Morelet infection in banana. Plant Disease 100, 1170-1175. https://doi.org/10.1094/PDIS-08-15-0950RE 
Rodríguez-García, C.M., Raigosa-Flores, N., Conde-Ferráez, L., Peraza-Echeverría, L., CantoCanché, B., James-Kay, A., 2006. Variation in electrophoretic karyotype among Mexican isolates of Mycosphaerella fijiensis. Canadian Journal of Plant Pathology 28, 236-241. https://doi.org/10.1080/07060660609507292

Rouxel, T., Grandaubert, J., Hane, J.K., Hoede, C., van de Wouw, A.P., Couloux, A., Dominguez, V., Anthouard, V., Bally, P., Bourras, S., Cozijnsen, A.J., Ciuffetti, L.M., Degrave, A., Dilmaghani, A., Duret, L., Fudal, I., Goodwin, S.B., Gout, L., Glaser, N., Linglin, J., Kema, G.H.J., Lapalu, N., Lawrence, C.B., May, K., Meyer, M., Ollivier, B., Poulain, J., Schoch, C.L., Simon, A., Spatafora, J.W., Stachowiak, A., Turgeon, B.G., Tyler, B.M., Vincent, D., Weissenbach, J., Amselem, J., Quesneville, H., Oliver, R.P., Wincker, P., Balesdent, M.-H., Howlett, B.J., 2011. Effector diversification within compartments of the Leptosphaeria maculans genome affected by Repeat-Induced Point mutations. Nat Commun 2, 202. https://doi.org/10.1038/ncomms 1189

Saintenac, C., Lee, W.-S., Cambon, F., Rudd, J.J., King, R.C., Marande, W., Powers, S.J., Bergès, H., Phillips, A.L., Uauy, C., Hammond-Kosack, K.E., Langin, T., Kanyuka, K., 2018. Wheat receptor-kinase-like protein Stb6 controls gene-for-gene resistance to fungal pathogen Zymoseptoria tritici. Nature Genetics 1. https://doi.org/10.1038/s41588-018-0051-x

Sánchez Timm, E., Hidalgo Pardo, L., Pacheco Coello, R., Chávez Navarrete, T., Navarrete Villegas, O., Santos Ordóñez, E., 2016. Identification of differentially-expressed genes in response to Mycosphaerella fijiensis in the resistant Musa accession 'Calcutta-4' using suppression subtractive hybridization. PLoS One 11. https://doi.org/10.1371/journal.pone.0160083

Santos, C.M.R., Martins, N.F., Hörberg, H.M., de Almeida, E.R.P., Coelho, M.C.F., Togawa, R.C., da Silva, F.R., Caetano, A.R., Miller, R.N.G., Souza, M.T., 2005. Analysis of expressed sequence tags from Musa acuminata ssp. burmannicoides, var. Calcutta 4 (AA) leaves submitted to temperature stresses. Theor. Appl. Genet. 110, 1517-1522. https://doi.org/10.1007/s00122-005-1989-5

Spanu, P.D., Abbott, J.C., Amselem, J., Burgis, T.A., Soanes, D.M., Stüber, K., Themaat, E.V.L. van, Brown, J.K.M., Butcher, S.A., Gurr, S.J., Lebrun, M.-H., Ridout, C.J., SchulzeLefert, P., Talbot, N.J., Ahmadinejad, N., Ametz, C., Barton, G.R., Benjdia, M., Bidzinski, P., Bindschedler, L.V., Both, M., Brewer, M.T., Cadle-Davidson, L., CadleDavidson, M.M., Collemare, J., Cramer, R., Frenkel, O., Godfrey, D., Harriman, J., Hoede, C., King, B.C., Klages, S., Kleemann, J., Knoll, D., Koti, P.S., Kreplak, J., LópezRuiz, F.J., Lu, X., Maekawa, T., Mahanil, S., Micali, C., Milgroom, M.G., Montana, G., Noir, S., O'Connell, R.J., Oberhaensli, S., Parlange, F., Pedersen, C., Quesneville, H., Reinhardt, R., Rott, M., Sacristán, S., Schmidt, S.M., Schön, M., Skamnioti, P., Sommer, H., Stephens, A., Takahara, H., Thordal-Christensen, H., Vigouroux, M., Weßling, R., Wicker, T., Panstruga, R., 2010. Genome expansion and gene loss in powdery mildew fungi reveal tradeoffs in extreme parasitism. Science 330, 1543-1546. https://doi.org/10.1126/science.1194573

Stergiopoulos, I., Burg, H.A. van den, Ökmen, B., Beenen, H.G., Liere, S. van, Kema, G.H.J., Wit, P.J.G.M. de, 2010. Tomato Cf resistance proteins mediate recognition of cognate homologous effectors from fungi pathogenic on dicots and monocots. PNAS 107, 7610-7615. https://doi.org/10.1073/pnas.1002910107

Stergiopoulos, I., Cordovez, V., Ökmen, B., Beenen, H.G., Kema, G.H.J., de Wit, P.J.G.M., 2014. Positive selection and intragenic recombination contribute to high allelic diversity in effector genes of Mycosphaerella fijiensis, causal agent of the black leaf streak disease of banana. Molecular Plant Pathology 15, 447-460. https://doi.org/10.1111/mpp.12104

Stergiopoulos, I., de Wit, P.J.G.M., 2009. Fungal effector proteins. Ann Rev Phytopathol 47, 233 263. https://doi.org/10.1146/annurev.phyto.112408.132637

Tabib Ghaffary, S.M., Robert, O., Laurent, V., Lonnet, P., Margalé, E., van der Lee, T.A.J., Visser, R.G.F., Kema, G.H.J., 2011. Genetic analysis of resistance to septoria tritici blotch in the French winter wheat cultivars Balance and Apache. Theor Appl Genet 123, 741-754. https://doi.org/10.1007/s00122-011-1623-7 
Teboul, L., Hérault, Y., Smith, C., Whitelaw, B., 2017. Introduction to Mammalian Genome Special Issue: Genome Editing. Mamm Genome 28, 235-236. https://doi.org/10.1007/s00335-0179708-5

Torres, J.M., Calderón, H., Rodríguez-Arango, E., Morales, J.G., Arango, R., 2012. Differential induction of pathogenesis-related proteins in banana in response to Mycosphaerella fijiensis infection. Eur J Plant Pathol 133, 887-898. https://doi.org/10.1007/s10658-012-0012-7

Torriani, S.F.F., Melichar, J.P.E., Mills, C., Pain, N., Sierotzki, H., Courbot, M., 2015. Zymoseptoria tritici: A major threat to wheat production, integrated approaches to control. Fungal Genetics and Biology, Septoria tritici blotch disease of wheat: Tools and techniques to study the pathogen Zymoseptoria tritici 79, 8-12. https://doi.org/10.1016/j.fgb.2015.04.010

Vilarinhos, A.D., Piffanelli, P., Lagoda, P., Thibivilliers, S., Sabau, X., Carreel, F., D'Hont, A., 2003. Construction and characterization of a bacterial artificial chromosome library of banana (Musa acuminata Colla). Theor Appl Genet 106, 1102-1106. https://doi.org/10.1007/s00122$002-1155-2$

Ware, S.B., 2006. Aspects of sexual reproduction in Mycosphaerella species on wheat and barley: genetic studies on specificity, mapping, and fungicide resistance ( $\mathrm{PhD}$ Thesis). Wageningen University and Research Centre, Wageningen, the Netherlands.

Wiame, L., Swennen, R., Sági, L., 2000. Pcr-based cloning of candidate disease resistance genes from banana (Musa acuminata), in: Acta Horticulturae. International Society for Horticultural Science (ISHS), Leuven, Belgium, pp. 51-58. https://doi.org/10.17660/ActaHortic.2000.521.3

Wicker, T., Oberhaensli, S., Parlange, F., Buchmann, J.P., Shatalina, M., Roffler, S., Ben-David, R., Doležel, J., Šimková, H., Schulze-Lefert, P., Spanu, P.D., Bruggmann, R., Amselem, J., Quesneville, H., Ver Loren van Themaat, E., Paape, T., Shimizu, K.K., Keller, B., 2013. The wheat powdery mildew genome shows the unique evolution of an obligate biotroph. Nature Genetics 45, 1092-1096. https://doi.org/10.1038/ng.2704

Yoshida, K., Saunders, D.G.O., Mitsuoka, C., Natsume, S., Kosugi, S., Saitoh, H., Inoue, Y., Chuma, I., Tosa, Y., Cano, L.M., Kamoun, S., Terauchi, R., 2016. Host specialization of the blast fungus Magnaporthe oryzae is associated with dynamic gain and loss of genes linked to transposable elements. BMC Genomics 17, 370. https://doi.org/10.1186/s12864-016-2690-6

Zaccaron, A.Z., Bluhm, B.H., 2017. The genome sequence of Bipolaris cookei reveals mechanisms of pathogenesis underlying target leaf spot of sorghum. Sci. Rep. 7, 17217. https://doi.org/10.1038/s41598-017-17476-x

Zandjanakou-Tachin, M., Ojiambo, P.S., Vroh-Bi, I., Tenkouano, A., Gumedzoe, Y.M., Bandyopadhyay, R., 2013. Pathogenic variation of Mycosphaerella species infecting banana and plantain in Nigeria. Plant Pathology 62, 298-308. https://doi.org/10.1111/j.13653059.2012.02650.x

Zhong, Z., Marcel, T.C., Hartmann, F.E., Ma, X., Plissonneau, C., Zala, M., Ducasse, A., Confais, J., Compain, J., Lapalu, N., Amselem, J., McDonald, B.A., Croll, D., PalmaGuerrero, J., 2017. A small secreted protein in Zymoseptoria tritici is responsible for avirulence on wheat cultivars carrying the Stb6 resistance gene. New Phytol 214, 619-631. https://doi.org/10.1111/nph.14434 


\section{Summary}

The hemibiotroph Pseudocercospora fijiensis is the causal agent of the black Sigatoka disease on bananas; present worldwide being the reason of large economical losses and subject to chemical control as the best control method nowadays. Nonetheless, this fungus has shown being able to become resistant to different chemical fungicides and thus a successful global threat to bananas. The biology of this fungus is largely unknown due to the missing -omics tools applied to this pathosystem. The only manner to counteract this fungus is to understand its biology of survival and pathogenicity.

Chapter 1. Is the introduction to the fungus, it constitutes an update to the knowledge of its biology and epidemiology. It demonstrates the importance of generating more data and tools to understand this powerful pathogen.

Chapter 2. Presents the $P$. fijiensis genome sequence, the analysis of its main characteristics compared with other close fungi. A new genetic map is included counting putative core and dispensable scaffolds, whose high polymorphism was observed by electrophoretic karyotyping within isolates from the same field population. It is shown the massive genome expansion mainly due to repetitive DNA, particularly by LTR-retrotransposons, and how this can affect close sequences. The effect of RIP on the genome is analyzed and compared to that on other closely related fungi. The location close to repetitive sequence from the effector Pfavr4 was discussed under the scope of its epidemiology and the protein employed helped to elucidate the first putative resistant cognate gene in the resistant banana cultivar Calcutta 4. Further analysis of strains originated from populations with and without fungicide selection pressure provided estimates of dispersal of strains and genetic flow that will help to predict spatial patterns of fungicide evolution under different management strategies.

Chapter 3. Describes the protocol for Agrobacterium-mediated transformation of P. fijiensis for both random and targeted mutagenesis. This method was successfully applied to the gene Pfavr4 and the Pfku70. The former is the first effector described in P. fijiensis, and the latter is the gene codifier of the KU70 protein, the main point of the non-homologous end joining (NHEJ) pathway that has been related to an increase on homologous recombination in several fungi and other eukaryotes, providing 
important tools for further use. This method was additionally employed to swap the Pfcyp51 gene promoter that helped to decipher biology of azole resistance in P. fijiensis, as described in chapter 4.

Chapter 4. The appearance of resistant strains to azoles has been mostly correlated with nonsynonymous point mutations in the coding sequence Pfcyp51 gene. In this chapter, we identified a 19 base pairs (bp) repeat element in the promoter region of this gene, by a simple PCR analysis, showed that copy number correlates positively with increased resistance to azoles, as well as the exposure to azole fungicides. We swapped the promoter of a resistant strain into a susceptible strain, and thus demonstrated that presence of the repeat element proportionally upregulates $P f c y p 51$ expression as well as tolerance to azoles. Besides the knowledge on genetic mechanism for azole resistance in $P$. fijiensis, the present study might offer another tool for optimizing the use of azoles in the control of black Sigatoka.

Chapter 5. A general discussion of the results obtained in this thesis is offered with a broader point of view. Implications in ecology, pathology and further expectations on the control of this fungus, together with insights on trending topics of molecular tools for future research are included. 


\section{Resumen}

El hemibiotrófico Pseudocercospora fijiensis es el agente causal de la enfermedad de la Sigatoka negra en banana; presente alrededor del mundo siendo la razón de grandes pérdidas económicas y sujeto a control químico como el mejor método de control hoy en día. Sin embargo, este hongo ha mostrado ser capaz de ser resistente a diferentes fungicidas químicos y así ser una exitosa amenaza mundial para el banano. La biología de este hongo es ampliamente desconocida debido a la carencia de herramientas ómicas aplicadas a este patosistema. La única manera de contrarrestar este hongo es entendiendo su biología de supervivencia y patogenicidad.

Capítulo 1. Es la introducción al hongo, constituye una actualización en el conocimiento de su biología y epidemiología. Demuestra la importancia de generar más datos y herramientas para entender este poderoso patógeno.

Capítulo 2. Presenta la secuencia genómica de $P$. fijiensis, el análisis de sus características principales comparadas con otros hongos filogenéticamente cercanos. Se incluye un nuevo mapa genético contando con putativos "scaffolds" principales y dispensables, cuyo alto grado de polimorfismo fue observado por cariotipo electroforético en aislados de una misma población. Se muestra también la expansión masiva del genoma debida principalmente al $\mathrm{ADN}$ repetitivo, particularmente por transposones tipo LTR y cómo éstos afectan secuencias vecinas. El efecto del RIP en el genoma es analizado y comparado con el de otros hongos filogenéticamente cercanos. La ubicación del efector Pfavr4 cercana a secuencias repetitivas fue discutida bajo el punto de vista de su epidemiología y la proteína empleada para elucidar el primer gen putativo cognado de resistencia en el cultivar de banana Calcuta 4. Análisis posteriores de líneas originarias de poblaciones diferentes con y sin selección de fungicida proporcionó la estimación de dispersión de éstas y flujo genético que ayudará a predecir patrones espaciales de evolución de resistencia a fungicidas bajo diferentes estrategias de manejo.

Capítulo 3. Describe el protocolo de transformación de P. fijiensis mediada por Agrobacterium para mutagénesis al azar y de genes blanco. Este método fue satisfactoriamente aplicado a los genes Pfavr4 y $P f k u 70$. El primero es el primer efector descrito en $P$. fijiensis y el segundo es el gen codificador 
para la proteína KU70, que es el punto principal de la ruta de unión de secuencias no homólogas (NHEJ, por sus siglas en inglés) que ha sido relacionada con un incremento en la recombinación homóloga en diversos hongos y otros eucariontes, proporcionando herramientas importantes para su uso posterior. Este método fue adicionalmente empleado para intercambiar el promotor del gen Pfcyp51 que ayudó a descifrar la biología de la resistencia a azoles en P. fijiensis, descrita en el capítulo 4.

Capítulo 4. La aparente resistencia de líneas del patógeno a los azoles ha sido correlacionada principalmente con mutaciones no sinónimas en la región codificante del gene Pfcyp51. En éste capítulo, nosotros identificamos un elemento repetitivo de 19 pares de bases (pb) en la región promotora de este gen, por un simple análisis de PCR, demostramos que el número de copias está correlacionado positivamente con un incremento en la resistencia a azoles, así como a la exposición a éstos. Intercambiamos el promotor de una línea resistente a una línea sensible y así demostramos que la presencia del elemento repetitivo regula proporcionalmente la expresión de Pfcyp51, así como la tolerancia a azoles. Además del conocimiento en el mecanismo genético de resistencia a azoles en $P$. fijiensis, el presente estudio podría ofrecer otra herramienta para optimizar el uso de azoles en el control de la Sigatoka negra.

Capítulo 5. Ofrece una discusión general de los resultados obtenidos en esta tesis dentro de un punto de vista más amplio. Se incluyen implicaciones en ecología, patogenicidad y expectativas a futuro en el control de este hongo, y una visión en tópicos actuales de herramientas moleculares para futuras investigaciones. 


\section{Acknowledgements}

Several times I wondered how I would write this piece of thesis pretending not to forget any single name. Now the time has come and today in a beautiful afternoon I am very glad to thank Jesus and Mary to be with me regardless of time, place and circumstances. I am deeply thankful to them for every second they spend with me and let me learn different lessons during my life in Wageningen and its people. Indeed, I grew up on the scientific life, but also as a person, and found that we are strong and rich depending on how much we want to trust.

I am grateful to the National Council of Science and Technology (CONACyT) from Mexico for the $\mathrm{PhD}$ scholarship, the kind support from Dioraphte Foundation and MusaRadix for the financial support.

My deepest gratitude to my supervisor and promotor Gert H.J. Kema, who trusted and supported me patiently all the time. I still remember when I met him in Merida, Mexico, where he showed to be a man of his word, since then I knew I have chosen good place to grow as scientist and I am proud of it; needless to say that I enjoyed the multicultural and multi religious environment with so much respect and friendliness.

Host pathogen interactions were my major curiosity before I came to Wageningen and I happily I ended up in the school of my co-promotor Prof. Pierre De Wit, to whom I am grateful for his valuable comments to my work. Besides, I remember how fascinating was to be at his class during my master studies, particularly when he arrived to classroom with a tree branch full of apple canker symptoms that he had just taken while cycling to class and excitedly explained its life cycle. Speaking to him was always to be in front of a book of phytopathology and life.

Special thanks to my co-promotor Prof. Rafael Arango, always supportive on site and in distance, very good scientist and honest person. I enjoyed so much his humor and friendly advising to everybody together with Esperanza, making an excellent pair in lab and life. 
At beginning of my PhD I was supervised by Manoel Souza from Embrapa Labex, to whom I am grateful for letting me know part of Brazilian culture, especially with the group of students and postdocs who came to the lab.

When very good results of this thesis were at the gate and founds were not abundant, continuation was largely because of Piet Boonekamp, manager of Bio-interactions business unit, Piet always showed a very positive side of scientific business and kindness to people specially during new year breakfasts with an open atmosphere. My sincere acknowledgement!

Likewise, I want to express my gratitude to MusaRadix B.V., which financed my stay as guess researcher while finishing the experiments of my thesis.

Special thanks to my colleagues at former Plant Research, particularly to those I worked close to, members of the former molecular phytopathology cluster and bio-interactions business unit, from whom I am grateful for brain storming, challenging my mind and scientific update on every meeting, besides were always helpful in technical issues, open for international traditions and kindly support on local customs to foreigners, you made the lab feel as home.

During my stay in the lab, I met numerous students who became friends. I am deeply thankful to Adilson Kobayashi from Embrapa. Adilson, you are a brilliant scientist and very kind person, you stepped in at the right moment with the right words and an open hand to help.

Unforgettable moments from the friendly working environment with Zhao Chunzhao, Sarrah Ben M`Barek, Sarah Ware, Rahim Mehrabi, Amir Mirzadi, Mahmod Tabib, Reza Talebi, Ulisses Nunes, Vivian Cordovez, Claudia Fortes, Tristan de Jong, Pablo Chong, Lamia Aouini and more recently also with Fernando García, Nadia Ordoñez, Maricar Salacinas, Rafael Segura and several other students that I probably miss here, nonetheless great memories filled my mind with special times.

Fernando, you were the first person to whom I showed lab issues, it was a great lab, greenhouse and office time besides your friendship! you quickly took your role and now I am happy for your achievements! 
Sarrah Ben M`Barek, you are a real royal friend, we shared several years of funny moments on everyday life and lab, traveling, congresses, faced long working hours, your enduring style to keep standing on any situation is admirable, although still I do not understand why a Rooibos tea can make you jump ;)

Pablo, it was a big pleasure to work with you, you are very simple and kind and above all funny to make the Spanglish a common language in the lab and strange drawings part of the office decoration. I am sure you will be very successful at work, as well as with your beautiful family.

Lamita, you were always so kind and interested on the human side of people, making you a wonderful friend. Although we ran the end of our thesis at same time and you are probably not there anymore, I very much hope to meet you in our beloved Wageningen. I have been extremely gifted by sharing our different believes, it is a gift to the world and I would love to let the world know differences enrich life. Thank you!

After several years in town, every corner of Wageningen became familiar and I met excellent friends not only in the lab. First of all, I thank Jan (q.e.p.d.) for his time to show me old people in need, encouraging words, example of strength and commitment.

My beloved friends from Franciscanos de María, who let me focus on what is important in life, with whom I shared and still share many common issues. Thank you! Likewise the international choir, you mean so much to me, a shelter, happy community, our bond was the unbreakable stone! In this time, thank you Francisco, Jaime and Ursula, Katarina, Myriam, Valentina, Xavier, Jose Luis, Luis, Filippo and Luisa, Hady and Jorge, Anita and Alvaro, Marie and Orda, Diego and Lupita, Ivo, AnneHendrike, Thilda, Michela and Yuri, Anna, Ameria, Alvaro, Ricardo, Rosio and many more generations of singers. How much and deeply enjoyed singing, our wonderful rehearsals, our first singing in Dutch language and the excursion to Makkum (hartelijk bedankt, Ivo!) are still present in my mind and heart... our bond was the unbreakable stone! I also thank F. Henry ten Have for such interesting homilies, the group of Shalom community always open for any help! Special thanks also to Stelita and Aad, Monika and Steve, Hady and Jorge: you made me part of your families, as simple and 
as much as that! Overall, I am deeply thankful to the International Catholic Community of Wageningen, that helped me to visualize a great focus in life.

Katarina, you were one of the first friends I had in town, in spite you knew I had come from Merida (-) I was gifted by your friendship, my "little hermanita" you were always taking rol on making new ones feel at home in a happy environment, shared many good and not very good times, covered me from circumstances I was blinded for and helped me when I most needed besides any situation you were facing... Djakuem!

In general, I am thankful to the town and university of Wageningen for keeping the environment so joyful and challenging that let me know many cultures and especially the Dutch; I think very high from this country with straightforward, transparent people, hard and efficient working style.

Close to Netherlands, but already in the German territories, Dr. Dieter Kaemmer supported me so much when I needed, I am so thankful for this and will never forget you introduced me into the banana world, and by helping Dr. Elizabeth Ortiz I was indeed helpt by both of you on professional things and received the privilege of your friendship.

Back in my country, I met also the wonderful Salvatorian community that coincides with my thoughts built for years from childhood to $\mathrm{PhD}$, on the best life approach and encouraged me to write this book, thank you Martin, Santiago, Pablo, Tomas, Salvador, Andres, Gloria, Carlos, Mónica, Gloria, Alfredo, Virginia, Caridad, Francisco, Maria Elena, Nidia. Likewise, I am thankful to all families Diaz and Trujillo for your love and care.

Last, but not least, the people who are the motor inside me, my parents. Because of them I am who and where I am. Papi, no tengo cómo devolverte los estudios que rechazaste para que yo naciera y naciera en la familia unida, más aún cada momento me sigues dando fortaleza y sabiduría para seguir adelante en los míos. Mamita, has sido fuerte, ejemplo de fé y ternura. Arnulfo, Jessy and León, you are very important part of this achievement. Les amo, son el regalo más grande que tengo y es para ustedes este libro. 


\section{About the author}

Caucasella Díaz Trujillo was born on November 19th, 1978 in Minatitlan, Mexico. She graduated as biologist at the Autonomous University of Yucatan (UADY) in 2003, working on the P. fijiensis banana pathosystem, under the guidance of Drs. Dieter Kämmer and Andrew James for her BSc thesis entitled "Obtaining microsatellite markers for Mexican lines of Mycosphaerella fijiensis", at the Center of Scientific Research of Yucatan (CICY). In 2004 she participated in the characterization of genomic library from a resistant banana, and started an awareness study for Fusarium oxysporum $\mathrm{f}$. sp. cubense (Foc) in local banana farms. In 2005 she obtained a scholarship of the National Council for Science and Technology of Mexico (CONACyT), and started her MSc studies in Plant Sciences at Wageningen University and Research (WUR). She focused, together with Dr. Sarah Ware, on Zymoseptoria tritici in wheat in the group of Prof. Gert H.J. Kema at Wageningen Plant Research. Her thesis "Biology of competition in Mycosphaerella graminicola: A molecular approach", described the determination of fungal biomass of Z. tritici during competition on wheat varieties with different resistance levels and under fungicide selection pressure. During her internship entitled "Functional validation of promoters in transgenic bananas" she started a survey of promoters of putative banana resistance genes at the Catholic University of Leuven in Belgium, in the group of Prof. Rony Swennen and under the guidance of Dr. Serge Remy. In 2007, Caucasella started her PhD on the interaction between P. fijiensis and banana with an extension of her CONACyT scholarship and in 2012-2013 she was involved as guest researcher, supported by MusaRadix B.V., in the initiation of the Panama disease program at WUR. Now, returned to Mexico, she is currently Phytopathology and Genetics Researcher at AgroStevia S.A.P.I. de CV. 


\section{Publications}

- Arango, R.E., Díaz-Trujillo, C., Dhillon, B., Aerts, A., Carlier, J., Crane, C.F., Jong, T.V. de, Vries, I. de, Dietrich, R., Farmer, A.D., Fortes Fereira, C., Garcia, S., Guzmán, M., Hamelin, R.C., Lindquist, E.A., Mehrabi, R., Quiros, O., Schmutz, J., Shapiro, H., Reynolds, E., Scalliet, G., Souza Jr, M., Stergiopoulos, I., Van der Lee, T.A.J., Wit, P.J.G.M. de, Zapater, M.-F., Zwiers, L.-H., Grigoriev, I.V., Goodwin, S.B., Kema, G.H.J., 2016. Combating a global threat to a clonal crop: the banana black Sigatoka pathogen Pseudocercospora fijiensis (synonym Mycosphaerella fijiensis) genomes reveals clues for disease control. PLoS Genetics 12(8): e1005876. doi: 10.1371/journal.pgen.1005876.

- Diaz-Trujillo, C., Chong, P., Stergiopoulos, I., Cordovez, V., Guzman, M., De Wit, P.J.G.M., Meijer, H.J.G., Scalliet, G., Sierotzki, H., Lilia Peralta, E., Arango Isaza, R.E., Kema, G.H.J., 2018. A new mechanism for reduced sensitivity to demethylationinhibitor fungicides in the fungal banana black Sigatoka pathogen Pseudocercospora fijiensis. Molecular Plant Pathology. https://doi.org/10.1111/mpp.12637

- Kema, G.H.J., Mirzadi Gohari, A., Aouini, L., Gibriel, H.A.Y., Ware, S.B., van den Bosch, F., Manning-Smith, R., Alonso-Chavez, V., Helps, J., Ben M'Barek, S., Mehrabi, R., Diaz-Trujillo, C., Zamani, E., Schouten, H.J., Van Der Lee, T., Waalwijk, C., De Waard, M.A., De Wit, P.J.G.M., Verstappen, E.C., Thomma, B.P.H.J., Meijer, H.J.G., Seidl, M.F., 2018. Stress and sexual reproduction affect the dynamics of the wheat pathogen effector AvrStb6 and strobilurin resistance | Nature Genetics. In press 


\title{
Education Statement of the Graduate School \\ Experimental Plant Sciences
}

\author{
Issued to: Caucasella Diaz Trujillo \\ Date: $\quad 6$ June 2018 \\ Group: Laboratorium of Phytopathology \& BU Biointeractions \\ University: Wageningen University \& Research
}
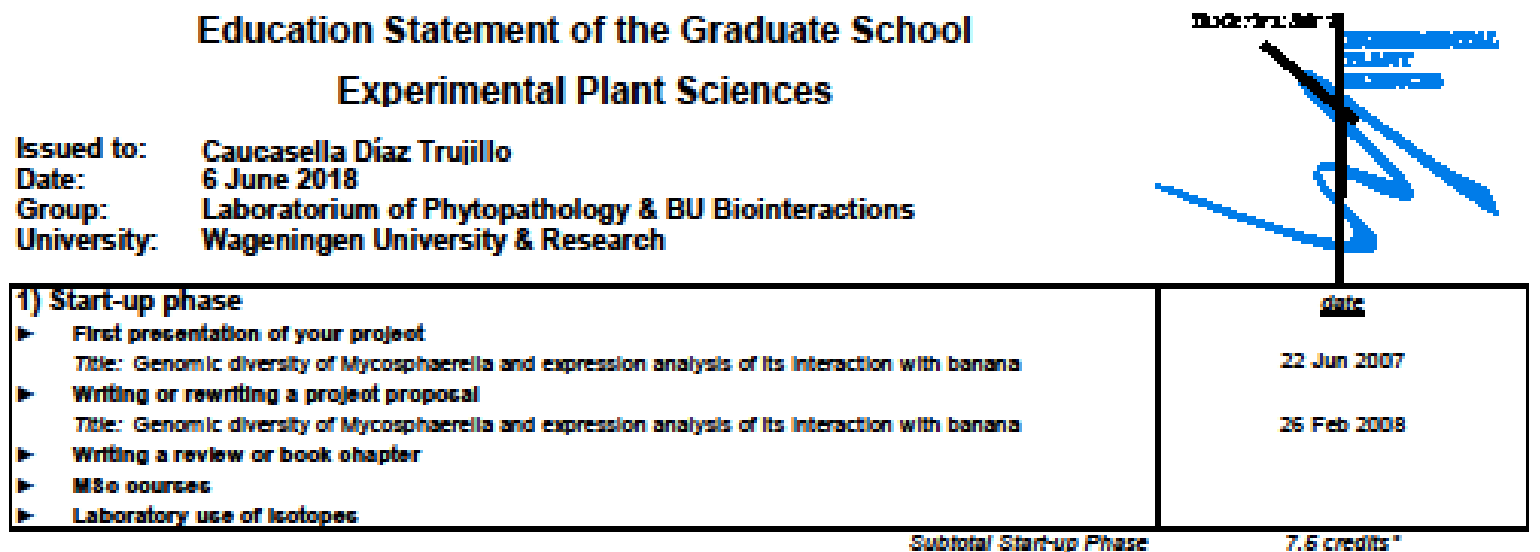

\begin{tabular}{|c|c|}
\hline 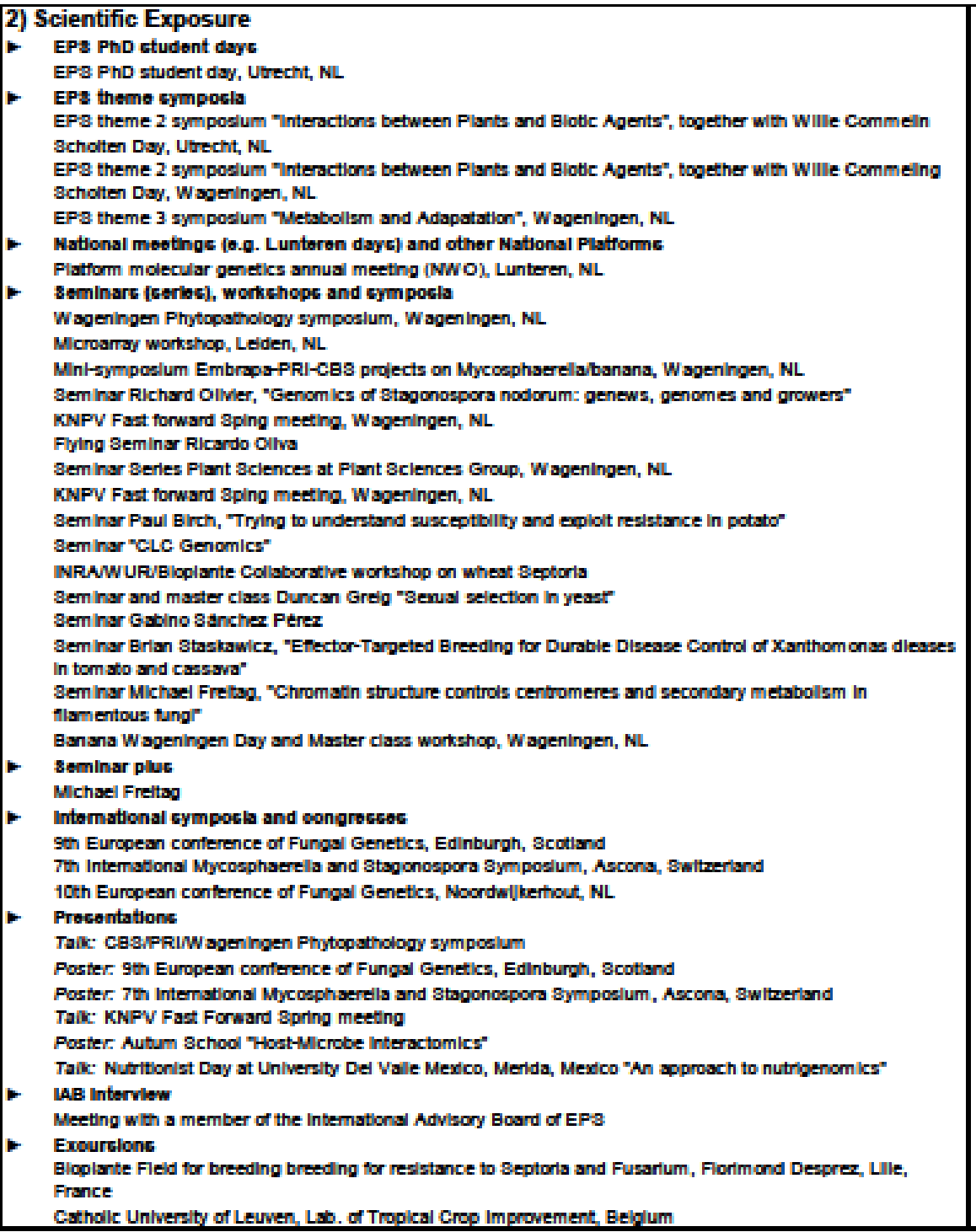 & 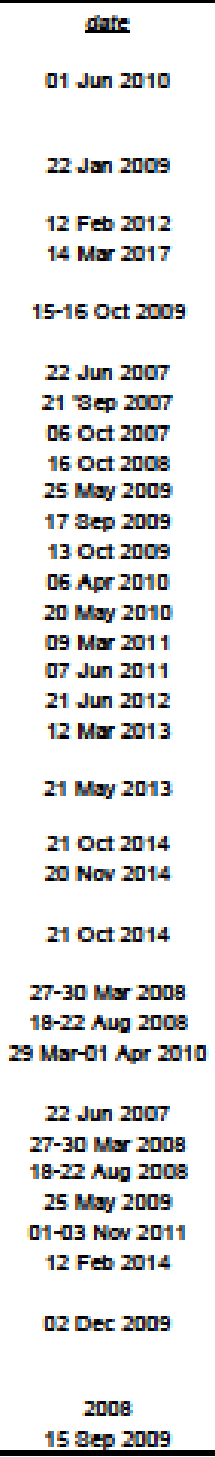 \\
\hline
\end{tabular}




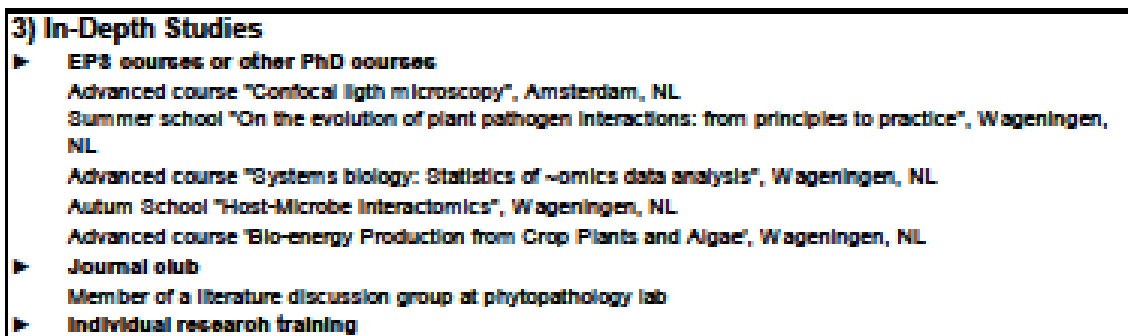

dinte

02-06 Jun 2008

18-20 Jun 2008

08-11 Dec 2008

01-03 Nov 2011

17-19 Nov 2014

$2007-2011$

8.6 credits $^{2}$

date

Bep-Nor 2009 $07-08$ Moy 2012

13 s 25 3ep 2011

3.9 credits $^{*}$

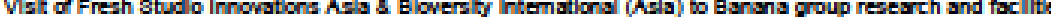
Uemberchlp of Board, Committoe or PhD oounoll

Herewith the Graduate school declares that the PhD candldate has compled with the educational requirements set

by the Educational Committee of EPs which comprises of a minimum total of 30 ECTs credita

-A credir represents a normative study lasd of 20 hours of study. 
The research described in this thesis was financially supported by Consejo Nacional de Ciencia y Tecnologia (CONACyT fellowship 187781, Mexico), MusaForever program and the Dioraphte Foundation grant 14.03.01.00

Financial support from Wageningen University, for printing this thesis is greatly acknowledged.

Cover design by Ligia Guadalupe Azueta Trujillo

Printed by Proefschriften 